\title{
Benjamini-Schramm Convergence of Normalized Characteristic Numbers of Riemannian Manifolds
}

\author{
Dissertation \\ ZUR ERLANGUNG DES MATHEMATISCH-NATURWISSENSCHAFTLICHEN \\ DOKTORGRADES
}

Doctor rerum naturalium

Der Georg-August-Universität GÖttingen

im Promotionsprogramm Mathematik Der Georg-August University SCHOOL OF SCIENCE (GAUSS)

VORGELEGT VON

Daniel Luckhardt

AUs MAINZ AM RHEIN 


\section{Remark}

In the revision of this thesis the proof of Lemma 3.9 turned out to be flawed. This affects Theorem 2.22 but not Theorem 2.27 These and all further statements affected have been indicated. 


\section{Betreuungsausschuss}

Prof. Dr. Thomas Schick

Prof. Dr. Ralf Meyer

\section{Mitglieder der Prüfungskommission}

Referent: Prof. Dr. Thomas Schick

Koreferent: Prof. Dr. Ralf Meyer

weitere Mitglieder der Prüfungskommission:

Prof. Dr. Stephan Huckemann

Prof. Ph.D. David Russell Luke

Prof. Dr. Victor Pidstrygach

Prof. Dr. Ingo Witt

Tag der mündlichen Prüfung: 5. Juni 2018. 


\begin{abstract}
We study a weak form of Gromov-Hausdorff convergence of Riemannian manifolds, also known as Benjamini-Schramm convergence. This concept is also applicable to other areas and has widely been studied in the context of graphs.

The main result is the continuity of characteristic numbers normalized by the volume with respect to the Benjamini-Schramm topology on the class of Riemannian manifolds with a uniform lower bound on injectivity radius and Ricci curvature. An immediate consequence is a comparison theorem that gives for any characteristic number a linear bound in terms of the volume on the entire class of manifolds mentioned. We give another interpretation of the result showing that characteristic numbers can be reconstructed with some accuracy from local random information.
\end{abstract}




\section{Achknowledgements}

My foremost thanks go to my supervisor Thomas Schick, who helped me with any mathematical problem, and thereby provided me any freedom I wanted, as well as looked after my financial support. I also want to thank the remaining members of my the examination board, especially my second supervisor Ralf Meyer.

The scientific environment at the mathematical institute in Göttingen I experienced will remain in my memory. The major part of this environment was the RTG Mathematical Structures in Modern Quantum Physics, which funded me for most of the time of my PhD studies until it cased to exist before this thesis was finished. Nevertheless, led by Dorothea Bahns, it provided me support in any direction I wanted to go. As an example of this support one should mention the course on the proof assistant Isabelle I was given the possibility to organize.

In Göttingen, I also enjoyed the company of Christoph Wockel, who brought me to the idea of applying the implicit function theorem to Banach spaces suitable for my investigations. Correspondence and conversation with Burkhard Wilking helped me on my path.

During my PhD I was often given the kind opportunity to give a talk on my research. In this regard I want to thank the Institut Fourier in Grenoble, Miklos Abert, Anand Dessai, Bernhard Hanke, and Jürgen Jost. The conversations I had with Miklos in Budapest on this occasion were quite insightful, though their fruits are not found in this thesis. To the same degree I enjoyed general discussions on Benjamini-Schramm convergence with Lewis Bowen, whose paper on Cheeger constants and L2-Betti numbers had brought me to the study of Benjamin-Schramm convergence of mm-spaces in the first place during my master thesis.

With regard to professional employment, I would like to thank Bernhard Hanke, who provied me with a position in Augsburg to finish this thesis, and Yair Glasner, who will provide me a postdoctoral fellowship at Ben-Gurion University for the forthcoming year.

It would be inappropriate to leave Ulrich Stuhler unmentioned, who not only provided me with letters of recommendation during my $\mathrm{PhD}$ but who had been a mathematical teacher of mine even since before I came to university. Finally, I want to thank my parents for their support. 


\section{Table of Contents}

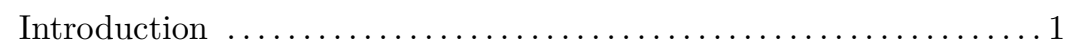

1 Preliminaries..$\ldots \ldots \ldots \ldots \ldots \ldots \ldots \ldots \ldots \ldots \ldots \ldots \ldots \ldots \ldots \ldots$

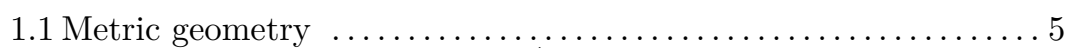

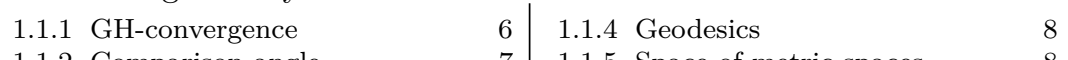

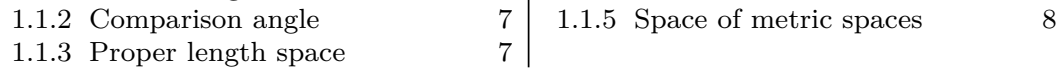

1.2 Metric measure spaces $\ldots \ldots \ldots \ldots \ldots \ldots \ldots \ldots \ldots \ldots \ldots \ldots$ \begin{tabular}{ll|l|l|l|}
1.2 .1 & Convergence of measures and & 1.2 .2 & Space of mm-spaces & 10 \\
\hline mm-spaces & 9 & 1.2 .3 & Measure theory & 11
\end{tabular}

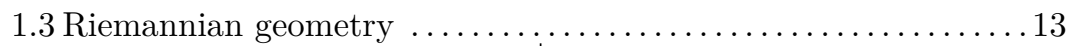

1.3 .1 Hölder regular functions 13

1.3 .2 Metric tensors of low

1.3.5 Fundamental Theorem of regularity

\begin{tabular}{l|l|l|l|l|l|}
\hline 1.3 .6 & Spaces of field spaces \\
\hline 1.3 .7 & Orientation and additional \\
\hline
\end{tabular}

Chart norms

1.3.4 Regularity of transition maps 18 measures

2 Main concepts and results. .25

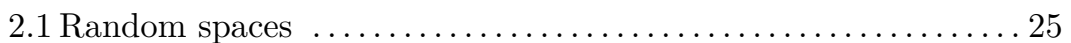

\begin{tabular}{ll|l|l|l|l|l|l|}
\hline 2.1 .1 & Benjamini-Schramm limits & 25 & 2.1 .3 & Parameters and Testability & 29
\end{tabular}

2.1 .2 Unimodular spaces

25 2.1.4 Random samples 29

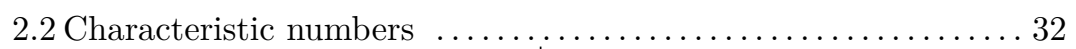

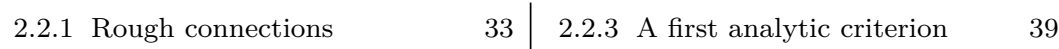

\begin{tabular}{ll|l|l|l|l|l|l|l|l|}
2.2 .2 & Testability from random & 2.2 .4 & A first geometric criterion 40
\end{tabular}

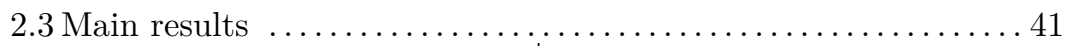

\begin{tabular}{|ll|ll|l|l|l|l|}
\hline 2.3 .1 & Main theorem and lemma & 42 & 2.3 .3 & Comparison theorem
\end{tabular}

2.3.2 Geometric versing

42

3 Proof of the main lemma..$\ldots \ldots \ldots \ldots \ldots \ldots \ldots \ldots \ldots \ldots \ldots$

3.1 Factorization of Hölder spaces $\ldots . \ldots \ldots \ldots \ldots \ldots \ldots \ldots \ldots . \ldots 45$

\begin{tabular}{ll|lll}
\hline 3.1 .1 & Tangent space factorization & 45 & theorem & 47
\end{tabular}

\begin{tabular}{|l|l|l|l|}
\hline 3.1 .2 & The implicit function & 49
\end{tabular}

3.2 Controlling coordinate charts $\ldots \ldots \ldots \ldots \ldots \ldots \ldots \ldots \ldots \ldots \ldots$

\begin{tabular}{ll|l|l|}
\hline 3.2 .1 & Strainers & 53 & 3.2 .3 Selection lemma \\
\hline 3.2 .2 & 57 & 60
\end{tabular}

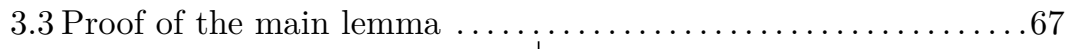

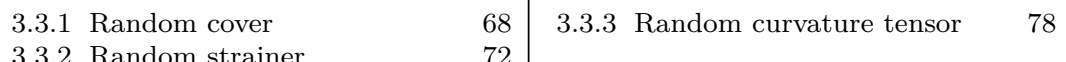

72

A Metrization and metrizability theorems $\ldots \ldots \ldots \ldots \ldots \ldots \ldots 87$

A.1 Spaces of pointed metric spaces $\ldots \ldots \ldots \ldots \ldots \ldots \ldots \ldots \ldots 87$

A.2 Space of pointed mm-spaces $\ldots \ldots \ldots \ldots \ldots \ldots \ldots \ldots \ldots$. . . . . . . . . . . 89

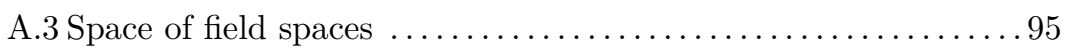

B Coordinate independence of rough curvature tensors. . . . . . . 103 


\section{Introduction}

The class of (equivalence classes up to isometry of) compact metric measure length spaces are denoted by $\mathbb{M}$. Additionally, let PM denote the class of pointed metric measure spaces (pointed mm-spaces) that are proper length spaces. We will often skip base point, metric and volume measure when denoting and element of this class, i.e. $M=(M, d, v o l, p)$. The main results of this thesis concerns a space $\mathrm{P} \mathcal{M}$ of (equivalence classes of) oriented pointed Riemannian manifolds. For pointed metric measure spaces there is the established notion of pointed Gromov-Hausdorff distance (PGH-distance). There are refinements for mm-spaces and oriented Riemannian manifolds, as explained in Sections 1.2 and 1.3

There is a weak, or probabilistic, notion of PGH-distance, given by the notion of Benjamini-Schramm covergence (BS-convergence). One can make sense of this concept in a far broader variety of contexts. The concept originated in the study of graphs (Benjamini and Schramm BS01, Aldous and Steele AS04, and Aldous and Lyons AL07]) and found vast application in this area, e.g. [Lyo05. BSS08, BL10; Bor+13 ATV17; AH15, Abé+16], where it is also called local weak convergence. The other two big areas of application are measured group theory 16, Gel15, entropy theory, and dynamical systems Bow17. In this context the contributions of a group of seven mathematician, calling themselves "Seven Samurai" on lattices in Lie groups, are to mention 7s11, 7s17, 7s16 as well as related results for arithmetic orbifolds [Rai13], and non-Archimedean local fields GL17].

Furthermore, there are occurrences in areas such as random matrix theory And17], simplicial complexes [Ele10], Riemannian manifolds AB16], or noncommutative probability Mal17. A major question that is underlying these investigations is whether in any context every unimodular space is sofic: A sofic space is a random space that can be described as the BS-limit of normal, i.e. deterministic, spaces of finite volume. Such spaces enjoy the property of being unimodular, a concept introduced in $\S 2.1 .2$ that can be formulated by the Intrinsic Mass-Transport-Principle stating that a point receives as much as it sends under transport. The arising question is whether every unimodular random space is sofic, i.e. a BS-limit of random spaces. Under appropriate definitions this question amounts in group theory to the question if every group is sofic (see also Remark 2.5, a big unsolved problem.

We will introduce BS-convergence in detail in Chapter 2, Let $\mathcal{P} \mathcal{X}$ denote the space of probability measures on a metric space $\mathcal{X}$ endowed with the topology of convergence against bounded continuous test functions.

Definition 0.1 (BS-convergence). Let $\mathbb{M}$ denote the set of (equivalence classes 
of) compact measure length spaces with finite measure. For any $M=(M, v o l) \in$ $\mathbb{M}$ let $\mu_{M}: M \rightarrow \mathrm{PM}$ be the map $p \mapsto(M, p, v o l)$ that assigns to each point a pointed version of $M$. Further let $\tilde{\mu}$ be the map

$$
\tilde{\mu}: \mathbb{M} \rightarrow \mathcal{P}(\mathrm{PM}), \quad M \mapsto\left(\mu_{M}\right)_{*}\left(\frac{v o l}{\operatorname{vol} M}\right)
$$

where $v o l$ is finite since $M$ is compact. We endow $\mathbb{M}$ with the topology induced by $\tilde{\mu}$ and the weak topology on $\mathcal{P}(\mathrm{PM})$. Likewise, we can say that a sequence $\left(M_{n}\right) \subset \mathbb{M}$ of spaces BS-converges if the laws $\tilde{\mu}_{n}(M)$ converge (against continuous test functions).

For the probabilistic formulation of BS-convergence, we first have to introduce the sample function on PM by

$$
s_{r}:(M, p) \mapsto(\mathrm{B}[p, r], p) .
$$

A sequence $\left(M_{n}\right.$, vol $\left._{n}\right) \in \mathbb{M}$ BS-converges if for random variables $X_{n}: \Omega \rightarrow M_{n}$ distributed according to $\operatorname{vol}_{n}$ the random variables

$$
\omega \mapsto s_{r}\left(M_{n}, X_{n}(\omega)\right) \in \mathrm{PM}
$$

converge in distribution for every $r \geq 0$.

By a parameter we will formally understand a partially defined real valued function on $\mathbb{M}_{\varphi} \subset \mathcal{M} \subset \mathcal{P} P \mathcal{M}$. Further we introduce the following Hungarian terminology (in normal statistics one would rather speak of "estimating" than of "testing" in the application given in Theorem 2.10 below)

Definition 2.6. A parameter is testable if it is continue and can be continued to the boundary of its domain.

Definition 2.7. A testable parameter is testable in constant time if its domain is relatively compact.

In this thesis we study the probably most investigated subclass of $\mathbb{M}$, Riemannian manifolds of some fixed dimension $d$. An important invariant in the investigation of (closed) oriented even-dimensional Riemannian manifold are Characteristic numbers. By so-called Chern-Weil theory they can be expressed by integration of certain forms on a manifolds. Each such class is described by a polynomial $\Pi$ on the ring $\mathrm{M}_{d}(\mathbb{C})$ invariant under base change. The invariant $\Pi[M]$ corresponding to $M$ is a complex number. It is possible to choose a base of characteristic numbers for $d$-dimensional manifolds such that the characteristic number of each base vector gives an integer, e.g. such a basis can be chosen in terms of Pontryagin numbers. However, we are interested in the normalized characteristic number

$$
\varphi_{\Pi}(M)=\frac{\Pi[M]}{\operatorname{vol}(M)} .
$$

Theorem 2.24 (Geometric Main Theorem). (Assuming the validity of Lemma 3.9) Let $\Pi$ be an invariant polynomial on $\mathrm{M}_{d}(\mathbb{C})$ and $i>0, \Lambda \in \mathbb{R}$. On the class of smooth d-dimensional oriented Riemannian manifolds $M$ satisfying

(i) Ric $M \geq-\Lambda$, 
(ii) $\operatorname{inj} M \geq i$

the parameter $\varphi_{\Pi}$ is testable in constant time.

A related result is:

Theorem 2.27 (volume comparison). Let $\Pi$ be an invariant polynomial on $\mathrm{M}_{d}(\mathbb{C}), i>0$, and $\Lambda \in \mathbb{R}$. There is a constant $C=C(\Pi, i, \Lambda)$ such that

$$
|\Pi[M]| \leq C \operatorname{vol}(M)
$$

for any closed Riemannian d-manifold with

(i) $\operatorname{Ric} M \geq-\Lambda$,

(ii) $\operatorname{inj} M \geq i$.

The quest for such bounds goes back to Cheeger and Gromov [CG85].

Crucial as well is the geometric motivation of this definition by the following theorem. This is that small changes of a space result only in small changes of the parameter, e.g. $\varphi_{\Pi}$. A probabilistic way to make this precise is as follows:

Theorem 2.10. Let $\varphi$ be a parameter that is testable in constant time. For any $\varepsilon>0$ there is a radius $r$ and a natural number $n$ and a tester $\tau$, i.e. a map $\tau:\left(s_{r}\left(\mathrm{PM}_{\varphi}\right)\right)^{n} \rightarrow \mathbb{R}$, such that the bound

$$
\operatorname{Prob}\left(\left|\varphi(M)-\tau\left(s_{r} X_{1}, \ldots, s_{r} X_{n}\right)\right|<\varepsilon\right)>1-\varepsilon \quad \text { for all }(M, d, \text { vol }) \in \mathbb{M}_{\varphi}
$$

holds, where $X_{1}, \ldots, X_{n}$ are uncorrelated random variables with law $\frac{1}{\operatorname{vol}(M)}$ vol.

This theorem is formulated for mm-spaces but holds as well for parameters on $\mathcal{P}(\mathrm{P} \mathcal{M})$ or also for other kinds of spaces, like simplicial complexes. To the knowledge of the author this is the first time that it is explicitly noted $X_{1}, \ldots, X_{n}$ have to be only uncorrelated and not independent.

Testability of normalized invariants holds for other invariants than characteristic numbers and also on different kinds of spaces: For a suitable class of mm-spaces, normalized Betti numbers are testable as shown by Bowen Bow15. Theorem 4.1] and [Luc14]. For simplicial complexes of bounded vertex degree Elek [Ele10] proved that Betti numbers normalized by the number of vertices are testable in constant time. This result was extended to the signature of $4 k$-dimensional triangulated manifolds in Luc11.

The first chapter summarized preliminaries on mm-spaces and Riemannian manifolds supplemented by a new economic explicit metrization of the space of isomorphism classes of mm-spaces using a generalized Wasserstein distance, Theorem 1.6, that is proved in appendix A.2 as a result in its own right. In Chapter 2 we explain the concept of Benjamini-Schramm convergence and our main result. The final chapter is devoted to the proof of the main lemma, Lemma 2.23. The idea of the proof is to choose charts in a controlled random way. 


\section{Chapter 1}

\section{Preliminaries}

In the first section we present some established underlying notions and theorems, many of which are found in the textbooks of Burago, Burago, and Ivanov BBI01. and Petersen [Pet16].

\subsection{Metric geometry}

In matters of metric geometry we follow mainly Burago, Burago, and Ivanov BBI01. In detail, we use the following conventions and definitions: The elementary notion is the notion of a (pointed) metric space, denoted by $(M, d)$, $\left(M^{\prime}, d^{\prime}\right)$ or $\left(N, d_{N}\right)\left((M, d, p),\left(M^{\prime}, d^{\prime}, p^{\prime}\right)\right.$ or $\left(N, d_{N}, q\right)$, respectively). Note that $d$ might have the value $\infty$. Usually, we write $M$ and $N$ suppressing the metric (and often also the base point). If no confusion can arise, the distance is also denoted by $|x y|=d(x, y)$ or, indicating the space, by $|x y|_{M}=d_{M}(x, y)$. We will also write

$$
a \wedge b:=\min \{a, b\} \quad \text { and } \quad a \vee b:=\max \{a, b\} .
$$

The class of maps $f: M \rightarrow N$ considered in this thesis will largely depend on the context; but it is called an embedding if it is distance preserving, i.e. $|f(x) f(y)|_{N}=|x y|_{M}$ for all $x, y \in M$; and it is called an isomorphism or an isometry if it is bijective and distance preserving.

By a notion of convergence on some set $\mathcal{X}$ we mean a predicate on $\mathcal{X}^{\mathbb{N}} \times \mathcal{X}$, i.e. a function $\mathcal{X}^{\mathbb{N}} \times \mathcal{X} \rightarrow\{$ false, true $\}$, that should be interpreted as saying whether or not a given sequence converges. The topology induced by a notion of convergence on $\mathcal{X}$ is defined by declaring a set $U$ to be open in $\mathcal{X}$ if for any $x \in U$ and any sequence $\left(x_{n}\right)_{n} \subset \mathcal{X}$ converging to $x$ it holds that all but finitely many $x_{i}$ lie in $U$. This definition guarantees that the intersection of finitely many open sets is open. In the other direction any topology or metric induces a notion of convergence by saying that a sequence converges to a point $x$ if for any neighborhood of $x$ all but finitely many elements of the sequence are contained in this neighborhood. Moreover we say that a sequence subconverges if it has a converging subsequence.

Lemma 1.1. Let $\mathcal{X}$ and $\mathcal{Y}$ be two spaces with a notion of convergence and $f: \mathcal{X} \rightarrow \mathcal{Y}$ be a set-theoretic map. If for any sequence $x_{n}$ converging to some $x$ 
with respect to the notion of convergence on $\mathcal{X}$ the image points $f\left(x_{n}\right)$ converge to $f(x)$ with respect to the notion of convergence on $\mathcal{Y}$, then the map $f$ is continuous with respect to the induced topologies on $\mathcal{X}$ and $\mathcal{Y}$.

Proof. Take any subset $V \subset \mathcal{Y}$ open in the induced topology on $\mathcal{Y}$. Further take any point $x \in f^{-1}(\mathcal{Y})$ and assume that there is some sequence $x_{n}$ converging to $x$ with respect to the notion of convergence on $\mathcal{X}$. Then $f\left(x_{n}\right)$ converges to $f(x)$, hence for sufficiently large $n$ the image points $f\left(x_{n}\right)$ are in $V$ and therefore $x_{n} \in f^{-1}(\mathcal{Y})$ for these $n$. This proves the claim.

Given a topological space $(\mathcal{X}, \mathcal{T})$ let $\mathcal{C}$ denote the induced notion of convergence. It follows from definition that any $\mathcal{T}$-open set is open in the topology induced by $\mathcal{C}$. Observe further

Lemma 1.2. In a metrizable topological space $(\mathcal{X}, \mathcal{T})$ a set $U$ is in $\mathcal{T}$ if and only if for every sequence converging to a point in $U$ with respect to the notion of convergence induced by $(\mathcal{X}, \mathcal{T})$ all but finitely many members already belong to $U$.

Proof. Fix a metric on $\mathcal{X}$ inducing $\mathcal{T}$. Let $\mathcal{C}$ denote the notion of convergence induced by $(\mathcal{X}, \mathcal{T})$. Further let $\mathcal{T}^{\prime}$ denote the topology induced by $\mathcal{C}$. Observe that as noted above $\mathcal{T} \subset \mathcal{T}^{\prime}$

For the non-trivial direction take a subset $U$ such that for every sequence $\left(x_{n}\right)_{n}$ converging with respect to $\mathcal{C}$ to a point $x \in U$ all but finitely many members are in $U$. Assume that $U$ does not belong to $\mathcal{T}$. By [Kel75, p. 119] a subset $U$ of a metric space is open with respect to the induced topology if and only if for each $x \in U$ there is a $\varepsilon>0$ such that the open ball of radius $\varepsilon$ around $x$ is contained in $U$. The assumption that $U$ is closed amounts to saying that there is a point $x \in U$ such that for each $\varepsilon>0$ there is a point $x_{\varepsilon}$ of distance less than $\varepsilon$ to $x$ such that $x_{\varepsilon} \notin U$. Take the sequence $\left(x_{1 / n}\right)_{n}$. We have $x_{1 / n} \stackrel{n \rightarrow \infty}{\longrightarrow} x$ and $x_{1 / n} \notin U$ for all $n$. This is in contradiction to our assumption.

\subsubsection{GH-convergence}

To establish a notion of convergence on the class of isometry classes of metric spaces, we agree on a further conventions. Closed balls and open balls in a metric space are denoted by

$$
\mathrm{B}[x, r]=\mathrm{B}_{M}[x, r] \quad \text { and } \quad \mathrm{B}(x, r)=\mathrm{B}_{M}(x, r),
$$

respectively, the index is suppressed if it is clear to which space $x$ belongs, e.g. $x=p$ or $M=\mathbb{R}^{d}$ if $x=0$. Sometimes we use even the shorthands $x^{r]}$ or $x^{r)}$. They are derived from the notations

$$
\begin{aligned}
& A^{r]}:=\{x \in M|\forall \varepsilon>0: \exists y \in A:| x y \mid \leq r+\varepsilon\} \quad \text { and } \\
& A^{r)}:=\{x \in M|\exists y \in A:| x y \mid<r\}
\end{aligned}
$$

for the closed and open thickening of some set $A \subset M$. Define its diameter by

$$
\operatorname{diam}(A):=\sup _{x, y \in A}|x y|
$$


The distortion of a map $f: M \rightarrow N$ is given by

$$
\operatorname{distor}(f):=\left.\sup _{x, y \in M}|| x y\right|_{M}-|f(x) f(y)|_{N} \mid \text {. }
$$

The Hausdorff distance of two closed subsets $A, B \subset M$ is given by

$$
\mathrm{d}_{\mathrm{H}}(A, B):=\inf \left\{r \in[0, \infty] \mid B \subset A^{r)} \text { and } A \subset B^{r)}\right\} .
$$

This can be generalized to the Gromov-Hausdorff distance (GH-distance) between two metric spaces

$$
\mathrm{d}_{\mathrm{GH}}(M, N):=\inf _{\iota, \eta} \mathrm{d}_{\mathrm{H}}(\iota(M), \eta(N))
$$

where the infimum is taken over all embeddings $\iota: M \rightarrow L$ and $\eta: N \rightarrow L$ in some metric space $L$, compare [BBI01, Definition 7.3.10]. The class of (isomorphism classes of) compact metric spaces forms a metric space with respect to $\mathrm{d}_{\mathrm{GH}}$. This space is complete as a consequence of BBI01, Theorem 7.4.15]. A sequence of pointed metric spaces $M_{n} \mathbf{P G H}$-converges to $M$ if for every $\varepsilon>0$ and $r>0$ there are for sufficiently large $n$ (not necessarily continuous) maps

$$
\iota_{n}: \mathrm{B}\left[p_{n}, r\right] \rightarrow M
$$

such that

$$
\iota_{n}\left(p_{n}\right)=p, \quad \operatorname{distor}\left(\iota_{n}\right) \leq \varepsilon, \text { and } \mathrm{B}[p, r-\varepsilon] \subset\left(\iota_{n} \mathrm{~B}\left[p_{n}, r\right]\right)^{\varepsilon)} .
$$

The functions $\iota_{n}$ are also called $\varepsilon$-isometries or comparison maps.

\subsubsection{Comparison angle}

Given three points $x, y$, and $z$ in $M$ such that $y$ is distinct from $x$ and $z$, we can assign to them the comparison angle at $y$ between $x$ and $z$

$$
\widetilde{\measuredangle}(x, y, z):=\arccos \frac{|x y|^{2}+|y z|^{2}-|x z|^{2}}{2|x y||z y|} .
$$

This definition is derived from the law of cosines. For a sequence $M_{n}$ converging to a space $M$ via some maps $\iota$ and points $x_{n}, y_{n}, z_{n}$, and $x, y, z$ it is immediate from definition that

$$
\left\{\begin{array}{l}
\iota x_{n} \rightarrow x \\
\iota y_{n} \rightarrow y \\
\iota z_{n} \rightarrow z
\end{array} \text { as } n \rightarrow \infty \text { implies } \widetilde{\measuredangle}\left(x_{n}, y_{n}, z_{n}\right) \rightarrow \widetilde{\measuredangle}(x, y, z) .\right.
$$

\subsubsection{Proper length space}

To each continuous path $\gamma: I \rightarrow M$ we can assign its length

$$
\text { length } \gamma:=\sup \left\{\sum_{i=1}^{n}\left|\gamma\left(x_{i-1}\right) \gamma\left(x_{i}\right)\right| \mid x_{0}, \ldots, x_{n} \in I, n \in \mathbb{N}\right\} \text {. }
$$

Further on the class of metric spaces there is the idempotent operation of forming the intrinsic metric

$$
\hat{d}(x, y):=\inf \{\text { length } \gamma \mid \gamma \text { is a path from } x \text { to } y\}
$$

for a metric space $(M, d)$ with the canonical continuous map $(M, \hat{d}) \rightarrow(M, d)$. A length space is a metric space such that this map is an isometry. 


\subsubsection{Geodesics}

In a length space a shortest path, or minimal geodesic, $\gamma: I \rightarrow M$ is a path $\gamma$ from $x$ to $y$ such that $d(x, y)=$ length $\gamma$ or, equivalently, for $I=[a, b]$ we have $d(\gamma(a), \gamma(t))=t-a$ BBI01, p. 48]. A metric space $M$ is proper if every bounded closed set is compact. Note that in this case $M$ is separable. It is a consequence of the Arzelà-Ascoli theorem that in a proper length space the distance of two points is always realized by a shortest path BBI01. Theorem 2.5.23]. Note that by the Hopf-Rinow-Cohn-Vossen theorem, [BBI01, Theorem 2.5.28], any locally compact complete length space is already proper.

In a length space a geodesic is a path $\gamma: I \rightarrow M$ such that $t \in I$ is contained in the interior of a subinterval $J=J_{t} \subset I$ such that $\left.\gamma\right|_{J}$ is a minimal geodesic. The injectivity radius at $x \in M, \operatorname{inj}_{x} M$, is defined by saying that $\operatorname{inj}_{x} M \geq r$ if all geodesics $\gamma, \gamma^{\prime}$ joining $x$ to some $y$ of length not greater than $r$ describe the same curve in $M$. Further set $\operatorname{inj} M:=\inf _{x} \operatorname{inj}_{x} M$. The injectivity radius is not continuous with respect to Gromov-Hausdorff convergence, as can be seen from the example:
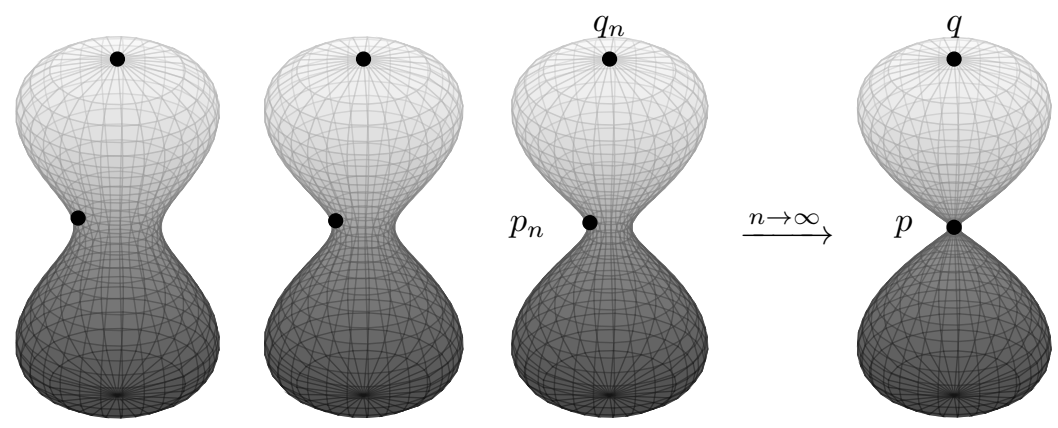

where $\operatorname{inj}_{p_{n}} M_{n} \rightarrow 0$ while two distinct geodesics starting at $p \in M$ can only intersect at the poles and any geodesic emitting from $q_{n}$ goes to the south pole without intersectiing any other geodesic emitting from $p_{n}$ while any two geodesics emitting from $q$ intersect at $p$.

\subsubsection{Space of metric spaces}

Let P $\mathcal{M}$ denote the class of (isomorphism classes of) pointed proper length spaces. This class can actually be represented as a set due to the separability statement of Theorem 1.3 below. As in the case of the space of close subspaces of a metric space there can be given a metrization, that is compatible with pointed Gromov-Hausdorff convergence. Define for two pointed spaces $(M, p)$ and $(N, q)$

$$
\begin{aligned}
\mathrm{d}_{\mathrm{PGH}}(M, N) & :=\sum_{r=1}^{\infty} r^{-2} \wedge \mathrm{d}_{\mathrm{PGH}}^{\prime}(\mathrm{B}[p, r], \mathrm{B}[q, r]) \\
\mathrm{d}_{\mathrm{PGH}}^{\prime}(M, N) & :=\inf _{\iota, \eta} \mathrm{d}_{\mathrm{H}}(\iota M, \eta N)+|\iota(p) \eta(q)|
\end{aligned}
$$

where the infimum is taken over all embeddings $\iota: \mathrm{B}[p, r] \rightarrow L$ and $\eta: \mathrm{B}[q, r] \rightarrow L$ to some compact metric space $L=(L,||$.$) . This definition gives a reasonable$ metric on $\mathrm{P} \mathcal{M}$. This fact is expressed by the following proposition proved in appendix A.1. 
Theorem 1.3. On PM a metric is given by $\mathrm{d}_{\mathrm{PGH}}$, that is complete, separable, and induces the same notion of convergence as defined by 1.2 and $(1.3)$.

Remark 1.4. The crucial definition 1.7a of $\mathrm{d}_{\mathrm{PGH}}$ metricizes PGH-convergence only if the domain is restricted to the class of length spaces, though this is sometimes forgotten in the literature. To see the problem, take a space $M=$ $(\{p, x\}, p)$ with $|p x|=1$. This space should be the limit of $M_{n}=\left(\left\{p, x_{n}\right\}, p\right)$ with $\left|p x_{n}\right|=1+1 / n$. But already for $r=1$ the summand in 1.7 a is equal to 1 for all $n$. Despite this fact, it is possible to define $\mathrm{d}_{\mathrm{PGH}}$ in a more refined way on the entire class of proper spaces.

\section{$1.2 \quad$ Metric measure spaces}

By a metric measure space, or mm-space, $M$ we understand a metric space that is in addition endowed with a boundedly finite measure, i.e. any bounded set has only finite measure. Note that in this case the measure is necessarily a Radon measure (every boundedly finite measure on a metric space is Radon Dud02, Theorem 7.1.3], i.e. it is inner regular, outer regular and locally finite). We denote the measure by vol and call it volume. Normally, these spaces will also be pointed and we will shortly write $M=(M, d$, vol, $p)$.

\subsubsection{Convergence of measures and mm-spaces}

Let $\mathcal{M} \mathcal{X}$ denote all boundedly finite measures on a metric space $\mathcal{X}$. Moreover let $\mathcal{P}(\mathcal{X})=\mathcal{P} \mathcal{X}$ denote all probability measures on $\mathcal{X}$. For probability measures we will also often use the term law. On both spaces we define topologies in terms of test functions $f: \mathcal{X} \rightarrow \mathbb{R}$

$$
\mu_{n} \rightarrow \mu \text { in } \mathcal{M X} \Longleftrightarrow \int f \mathrm{~d} \mu_{n} \rightarrow \int f \mathrm{~d} \mu
$$

for all bounded continuous $f$ with bounded support

for all bounded continuous $f$.

$$
P_{n} \rightarrow P \text { in } \mathcal{P} \mathcal{X} \Longleftrightarrow \int f \mathrm{~d} \mu_{n} \rightarrow \int f \mathrm{~d} \mu
$$

The former notion of convergence is often called weak ${ }^{\#}$ convergence. The latter is called weak convergence or simply convergence in law. If $\mathcal{X}$ is a complete separable metric space, so $\mathcal{M} \mathcal{X}$ is completely metrizable and separable DV03, Theorem A2.6.III(i)]. Moreover for such $\mathcal{X}$ the topology obtained by restricting $\mathcal{M} \mathcal{X}$ to $\mathcal{P} \mathcal{X}$ coincides with the topology of law convergence (e.g. as a direct consequence of [DV03, Proposition A2.6.II(iii)]). Restriction of a measure $\mu$ on $\mathcal{X}$ to a Borel set $A \subset \mathcal{X}$ by

$$
\left.\mu\right|_{A} \text { and } \mu \cap A \text {. }
$$

Denote by PM the class of (isomorphism classes of) proper pointed metric measure spaces $M=(M, d, p, v o l)$ that are length spaces. To define a suitable metric on PM, first define the bump function $b_{x, r}$ by

$$
b_{x, r}(y)= \begin{cases}1 & \text { if }|x y| \leq r-1 \\ r-|x y| & \text { if } r-1 \leq|x y| \leq r \\ 0 & \text { if }|x y| \geq r\end{cases}
$$


We say that a sequence $\left(M_{n}\right)_{n} \subset \mathrm{PM}$ does Pmm-converge to $M$ if for all $r>0$ and $\varepsilon>0$ there are for sufficiently large $n$ measurable maps

$$
\iota_{n}: \mathrm{B}\left[p_{n}, r\right] \rightarrow M
$$

such that 1.3 holds and $\iota_{n *}\left(b_{p_{n}, r}(\right.$.$\left.) vol _{n}\right)$ weak ${ }^{\#}$ converges to $b_{p, r}$ vol.

A further generalization, that is necessary due to technical reasons, are pointed mm-spaces with $k$-measures $\left(M, d, p, v_{1}, \ldots, v_{1} l_{k}\right)$ for some $k=$ $1,2, \ldots$. The definition of convergence of mm-spaces is to be read mutatis mutandis, i.e. weak ${ }^{\#}$ convergence of $\iota_{n *}\left(b_{p_{n}, r}(.) \operatorname{vol}_{i n}\right)$ to $b_{p, r}(.) \operatorname{vol}_{i}$ for all $i=1, \ldots, k$ is required. Let $\mathrm{PM}^{[k]}$ denote the space of (equivalence classes of) pointed proper length spaces with $k$ measures.

\subsubsection{Space of $\mathrm{mm}$-spaces}

Let PM denote the class of pointed mm-spaces $(M, d$, vol, $p)$ such that $(M, d, p)$ is in $\mathrm{P} \mathcal{M}$, i.e. it is a proper length mm-spaces, and $v o l \in \mathcal{M}(M)$. A metrization of the class of arbitrary proper mm-spaces with $k$-measures by a separable complete metric is stated in Bowen Bow15, Theorem 3.1]. On the space PM ${ }^{[k]}$ of pointed mm-spaces with $k$ measures we introduce the following alternative shorter metrization:

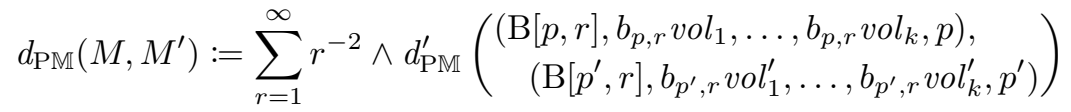

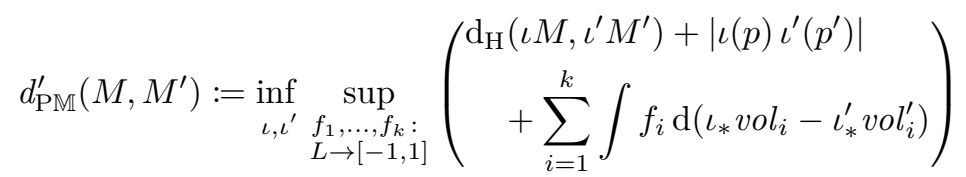

where the infimum is taken over all embeddings $\iota: M \rightarrow L$ and $\iota^{\prime}: M^{\prime} \rightarrow L$ to some compact metric space $L=(L,||$.$) , like in 1.7 \mathrm{~b}$; and the supremum is taken over all Lipschitz functions $f: L \rightarrow[-1,1]$ with Lipschitz constant not greater than 1 .

Remark 1.5 (Wasserstein distance). The term introduced for each measure is the dual representation of the Wasserstein metric given by the KantorovichRubinstein theorem for arbitrary measures: The well established Wasserstein distance on the laws on a metric space $(M, d)$ is the given by [Dud02, p. 420]

$$
W(P, Q)=\inf _{\mu} \int d(x, y) \mathrm{d} \mu
$$

where the infimum is taken over all laws on $M \times M$ such that the marginals, i.e. the push-forwards along both projections, are $P$ and $Q$. By the well-known Kantorovich-Rubinstein theorem [Dud02, Theorem 11.8.2] this quantity can equivalently be calculated by

$$
\sup _{f} \int f \mathrm{~d}(P-Q)
$$

where the supremum is taken over all Lipschitz functions $f: M \rightarrow[-1,1]$ with Lipschitz constant not greater than 1. 
The Wasserstein distance can be generalized for arbitrary measures $\mu$ and $\nu$ on a metric space $M$. First note that the classic definition of Wasserstein distance $W$ works fine for measures of same mass. Further let the mass $|\mu|$ of a signed measures $\mu=\mu^{+}-\mu^{-}$be $\mu^{+}(M)+\mu^{-}(M)$. Define

$$
\tilde{W}(\mu, \nu):=\inf _{\substack{\tilde{\mu}, \tilde{\nu} \in \mathcal{M} M \\|\tilde{\mu}|=|\tilde{\nu}|}}|\mu-\tilde{\mu}|+|\nu-\tilde{\nu}|+W(\tilde{\mu}, \tilde{\nu}) .
$$

This metric was introduced by Piccoli and Rossi $[\overline{P R} 14]$. In $[\mathrm{PR} 16]$ these authors prove the Kantorovich-Rubinstein theorem for the generalized Wasserstein distance, i.e. $\tilde{W}$ can be calculated by 1.13 — note that in PR14; PR16 only measures on $\mathbb{R}^{d}$ are considered but the proof of the Kantorovich-Rubinstein theorem therein works for any locally compact metric spaces.

Theorem 1.6. On $\mathrm{PM} \mathbb{M}^{[k]}$ a metric is given by $d_{\mathrm{PM}[k]}$, that is complete, separable, and induces the same notion of convergence as defined by 1.11.

Let PPM ${ }^{[k]}$ denote the set of (isomorphism classes of) doubly pointed mmspaces, i.e. spaces with two distinguished points. A complete and separable metric on this space is given by

$$
\begin{aligned}
& d_{\mathrm{PPM}}\left((M, \overrightarrow{v o l}, p, q),\left(M^{\prime}, \overrightarrow{v^{\prime} l^{\prime}}, p^{\prime}, q^{\prime}\right)\right) \\
&:=|| p q|-| p^{\prime} q^{\prime}||+d_{\mathrm{PM}[k+1]}\left(\left(M, \overrightarrow{v o l}, \delta_{q}, p\right),\left(M^{\prime}, \overrightarrow{v^{\prime} l^{\prime}}, \delta_{q^{\prime}}, p^{\prime}\right)\right) \\
&+d_{\mathrm{PM}[k+1]}\left(\left(M, \overrightarrow{v o l}, \delta_{p}, q\right),\left(M^{\prime}, \overrightarrow{v^{\prime} l^{\prime}}, \delta_{p^{\prime}}, q^{\prime}\right)\right)
\end{aligned}
$$

where $\delta_{p}$ etc. are Dirac measures and $\overrightarrow{v o l}=\left(v_{0} l_{1}, \ldots, v_{k}\right)$ abbreviates the collection of measures. The definition uses the $d_{\mathrm{PM}[k+1]}$ twice simply to guarantee symmetry.

\subsubsection{Measure theory}

Lemma 1.7 (integration lemma). Let $\mathcal{X}$ and $\mathcal{Y}$ be complete separable metric spaces. Let $m_{(.)}: \mathcal{X} \rightarrow \mathcal{M Y}$ be a map continuous with respect to weak ${ }^{\#}$ convergence on $\mathcal{M Y}$. For any law $P$ on $\mathcal{X}$ and any bounded Borel set $A \subset \mathcal{Y}$ the integral

$$
Q(P): A \mapsto \int_{\mathcal{X}} m_{x}(A) \mathrm{d} P(x)
$$

is defined. This assignment determines a measure $Q(P)$ on $\mathcal{Y}$. Further,

(i) if $m_{(.)}(A)$ is uniformly bounded for any bounded Borel set $A$, then the assignment $P \mapsto Q(P)$ is continuous with respect to convergence of laws and weak ${ }^{\#}$ convergence;

(ii) if the codomain of $m_{(.)}$actually restricts to $\mathcal{P} \mathcal{Y}$, then $Q(P)$ is a law;

(iii) if the integral $\int f \mathrm{~d} Q(P)$ is defined for a function $f: \mathcal{Y} \rightarrow[-\infty, \infty]$ it can be calculated by

$$
\int f \mathrm{~d} Q(P)=\iint f(y) \mathrm{d} m_{x}(y) \mathrm{d} P
$$


Proof. The Borel $\sigma$-algebra of $\mathcal{M Y}$ is the smallest $\sigma$-algebra for which the evaluation functions $\mu \mapsto \mu(A)$ is measurable for all bounded $A$ DV03, Theorem A2.6.III(iii)]. Being continuous the assignment $x \mapsto m_{x}$ is measurable, hence the concatenation $x \mapsto m_{x}(A)$ is measurable and $Q(P)(A)$ exists. Also by DV03, Theorem A2.6.III(iii)] the bounded Borel sets form a semiring (i.e. a system of subsets containing the empty set, closed under finite intersections, and with the property that for any two sets $U, V$ we have $U \backslash V$ is the union of finitely many disjoint sets of the system) that generates the Borel $\sigma$-algebra of $\mathcal{M Y}$. By the monotone convergence theorem the assignment $Q(P)$ is countably additive on this semiring. Hence Dud02, Proposition 3.2.4] $Q$ extends to a measure on he Borel $\sigma$-algebra of $\mathcal{M Y}$.

Claim (iii) is stated by Fremlin [Fre03, par. 452F]. For claim (i) assume that $m_{(.)}(A)$ is uniformly bounded for any bounded Borel set $A$. The integral $\int f \mathrm{~d} Q(P)$ is defined for any continuous bounded $f$ with bounded support. Note that the map $x \mapsto \int f(y) \mathrm{d} m_{x}(y)$ is continuous as a concatenation of $m_{(.)}$and evaluation on $f$ as well as bounded in absolute value by $\sup _{x, y}|f(y)| m_{x}(\operatorname{supp} f)$ due to our assumption of a uniform bound. Hence, for any convergent sequence $P_{n} \rightarrow P$ we observe $\int f \mathrm{~d} Q\left(P_{n}\right)=\iint f(y) \mathrm{d} m_{x}(y) \mathrm{d} P_{n}$ converges to $\iint f(y) \mathrm{d} m_{x}(y) \mathrm{d} P=\int f \mathrm{~d} Q(P)$. This is to say that $Q$ is continuous.

For claim (ii) insert $\mathcal{Y}$ and observe that $Q(P)(\mathcal{Y})=\int 1 \mathrm{~d} P=1$ assuming that $m_{(.)}$is valued in $\mathcal{P} \mathcal{Y}$.

The support of a measure $\mu$ on $\mathcal{X}$ is the closed set defined as

$$
\operatorname{supp} \mu:=\bigcap_{\substack{A \subset \mathcal{X} \text { closed, } \\ \mu(\mathcal{X} \backslash A)=0}} A .
$$

Recall that a Polish space is a separable completely metrizable space. By a result of Giry Gir82 we have:

Lemma 1.8 (Giry monad). The assignment $\mathcal{X} \mapsto \mathcal{P} \mathcal{X}$ of a Polish space $\mathcal{X}$ to the space of Borel probability measures on $\mathcal{X}$ with convergence in law forms a monad. On maps $f: \mathcal{X} \rightarrow \mathcal{Y}$ the monad is described by

$$
P \mapsto\left(f \mapsto \int_{\mathcal{X}} h \circ f\right)
$$

for any law $P \in \mathcal{P}(\mathcal{X})$ and bounded continuous function $h: \mathcal{Y} \rightarrow \mathbb{R}$.

Unit and multiplication of the monad are given by

$$
\begin{array}{rlrl}
\eta_{\mathcal{X}}: \mathcal{X} \rightarrow \mathcal{P}(\mathcal{X}), & & \mapsto \mapsto \delta_{x}, \\
\mu_{\mathbb{X}}: \mathcal{P} \mathcal{P}(\mathcal{X}) \rightarrow \mathcal{P}(\mathcal{X}), & P \mapsto\left(A \mapsto \int Q(A) \mathrm{d} P(Q)\right)
\end{array}
$$

for any Borel set $A \subset \mathcal{X}$ and $\delta_{x}$ the Dirac measure on $\mathcal{X}$.

Remark 1.9 (categorical properties of probability measures). The operation $Q$ from Lemma 1.7 actually can be described as $f \mapsto \mu_{\mathcal{P}} \mathcal{Y} \circ(\mathcal{P}(f))$. Moreover the map $f$ is a morphism in the Kleisli category belonging to the Giry monad. There is also a version of the Giry monad for complete separable metric spaces, called Kantorovic monad [FP17. 


\subsection{Riemannian geometry}

As for Riemannian geometry, we mostly follow Petersen Pet16]. It is fundamental to our approach to view a Riemannian manifold as an mm-spaces, i.e.

$$
(M, g, p)=\left(M, d_{g}, \text { vol }_{g}, p\right) \in \mathrm{PM} .
$$

The metric tensor $g$ will not be assumed to be smooth but only of some Hölder regularity. For a treatment of convergence theory for smooth tensors see Lessa Les15 and Abert and Biringer AB16.

\subsubsection{Hölder regular functions}

Let $f$ be a real valued function on some open ball $\mathrm{B}(0, \varrho) \subset \mathbb{R}^{d}$ (for simplicity we restrict to Euclidean balls, but the theory can be extended to other open domain in $\mathbb{R}^{d}$ ) and $m=0,1, \ldots$ an integer. Recall that, provided that all derivatives of $f$ up to order $m$ exist, the possibly infinite $\mathrm{C}^{m}$-norm is given by

$$
\|f\|_{\mathrm{C}^{m}}:=\sum_{0 \leq|a| \leq m} \sup _{x \in \mathrm{B}(0, \varrho)}\left|\partial^{a} f(x)\right|
$$

where $a=\left(a_{1}, \ldots, a_{d}\right)$ is a multi-index and $|a|=a_{1}+\ldots+a_{d}$ is its order. The Hölder semi-norm is given by

$$
\|f\|_{\alpha}:=\sup _{x, y \in \mathrm{B}(0, \varrho)} \frac{|f(x)-f(y)|}{|x-y|^{\alpha}} .
$$

This allows to define the $\mathrm{C}^{m, \alpha}$-Hölder-norm, or shortly $\mathrm{C}^{m, \alpha}$-norm, for $\alpha \in(0,1]$ by

$$
\|f\|_{\mathrm{C}^{m, \alpha}}:=\|f\|_{\mathrm{C}^{m}}+\sum_{|a|=m}\left\|\partial^{a} f\right\|_{\alpha} .
$$

If $\alpha>0$ and $\|f\|_{\mathrm{C}^{m, \alpha}}<\infty, f$ and its derivatives up to order $m$ can be uniquely continued to the boundary. Hence we denote the space of such functions by $\mathrm{C}^{m, \alpha}(\mathrm{B}[0, r])$. If, on the other hand, the $\mathrm{C}^{m, \alpha}$-norm is only bounded on each compact set strictly contained in the domain, we call $f$ a $\mathrm{C}^{m, \alpha}$-function or of class $\mathrm{C}^{m, \alpha}$. Finally, we set

$$
\begin{aligned}
\mathrm{C}^{m}(\ldots) & :=\mathrm{C}^{m, 0}(\ldots) \\
\mathrm{C}_{0}^{m, \alpha}(\ldots) & :=\left\{f \in \mathrm{C}^{m, \alpha}(\ldots) \mid f \text { has compact support }\right\} \\
\|f\|_{\alpha} & :=\left\|f_{1}\right\|_{\alpha}+\ldots+\left\|f_{n}\right\|_{\alpha} \\
\|f\|_{\mathrm{C}^{m, \alpha}} & :=\left\|f_{1}\right\|_{\mathrm{C}^{m, \alpha}}+\ldots+\left\|f_{n}\right\|_{\mathrm{C}^{m, \alpha}}
\end{aligned}
$$

for a vector valued function $f: \mathrm{B}(0, \varrho) \rightarrow \mathbb{R}^{n}$.

A comprehensive introduction to Hölder functions including all basic facts used in this thesis is given by Csató, Dacorogna, and Kneuss [CDK11, Chapter 16]. Standard references on this topic also include Gilbarg and Trudinger GT77. Throughout this thesis, we will take extensive usage of the following estimates:

Lemma 1.10 ([CDK11, Theorem 16.28]). Let $\varrho>0, m \geq 0$ an integer, and $\alpha \in[0,1]$. Then there exists a constant $C=C(\varrho, m)>0$ such that

$$
\|f g\|_{\mathrm{C}^{m, \alpha}} \leq C\left(\|f\|_{\mathrm{C}^{m, \alpha}}\|g\|_{\mathrm{C}^{0}}+\|f\|_{\mathrm{C}^{0}}\|g\|_{\mathrm{C}^{m, \alpha}}\right)
$$

for functions $f, g \in \mathrm{C}^{m, \alpha}(\mathrm{B}[0, \varrho], \mathbb{R})$. 
Corollary 1.11. Under the same assumptions as in Lemma 1.10 and $C=$ $C(\varrho, m)$

$$
\|f g\|_{\mathrm{C}^{m, \alpha}} \leq C\|f\|_{\mathrm{C}^{m, \alpha}}\|g\|_{\mathrm{C}^{m, \alpha}}
$$

for functions $f, g \in \mathrm{C}^{m, \alpha}(\mathrm{B}[0, \varrho], \mathbb{R})$.

Lemma 1.12 ([CDK11, Proposition 16.30]). Let $\varrho>0, m \geq 0$ an integer, and $\alpha \in[0,1]$. Further let $A \in \mathrm{C}^{m, \alpha}\left(\mathrm{B}[0, \varrho], \mathbb{R}^{n \cdot n}\right)$ be a matrix valued function and $c>0$ such that

$$
\left\|\frac{1}{\operatorname{det} A}\right\|_{\mathrm{C}^{0}} \leq c \text { and }\|A\|_{\mathrm{C}^{0}} \leq c .
$$

Then there exists a constant $C=C(c, \varrho, m)>0$ such that

$$
\left\|A^{-1}\right\|_{\mathrm{C}^{m, \alpha}} \leq C\|A\|_{\mathrm{C}^{m, \alpha}} .
$$

In particular, if there exists a constant $c>0$ so that

$$
\left\|A^{-1}\right\|_{\mathrm{C}^{0}} \leq c \quad \text { and } \quad\|A\|_{\mathrm{C}^{0}} \leq c
$$

then there exists a constant $C=C(c, \varrho, m)>0$ such that

$$
\left\|A^{-1}\right\|_{\mathrm{C}^{m, \alpha}} \leq C\|A\|_{\mathrm{C}^{m, \alpha}} \text {. }
$$

Lemma 1.13 ([CDK11, Theorem 16.31]). Let $\varrho, \varrho^{\prime}>0, m \geq 0$ an integer, and $\alpha, \beta \in[0,1]$. Further let $g \in \mathrm{C}^{m, \alpha}\left(\mathrm{B}\left[0, \varrho^{\prime}\right], \mathbb{R}\right)$.

If $m=0$ and $f \in \mathrm{C}^{\beta}\left(\mathrm{B}_{\mathbb{R}^{d}}[0, \varrho], \mathbb{R}^{d^{\prime}}\right)$ with $f\left(\mathrm{~B}_{\mathbb{R}^{d}}[0, \varrho]\right) \subset \mathrm{B}\left[0, \varrho^{\prime}\right]$, then

$$
\|g \circ f\|_{\mathrm{C}^{m, \alpha}} \leq\|g\|_{\mathrm{C}^{\alpha}}\|f\|_{\mathrm{C}^{\beta}}+\|g\|_{\mathrm{C}^{0}} .
$$

If $m \geq 1$ and $f \in \mathrm{C}^{m, \alpha}\left(\mathrm{B}_{\mathbb{R}^{d}}[0, \varrho], \mathbb{R}^{d^{\prime}}\right)$ with $f\left(\mathrm{~B}_{\mathbb{R}^{d}}[0, \varrho]\right) \subset \mathrm{B}\left[0, \varrho^{\prime}\right]$, then there is a constant $C=C\left(m, \varrho, \varrho^{\prime}\right)$ such that

$$
\|g \circ f\|_{\mathrm{C}^{m, \alpha}} \leq C\left(\|g\|_{\mathrm{C}^{m, \alpha}}\|f\|_{\mathrm{C}^{1}}^{m+\alpha}+\|g\|_{\mathrm{C}^{1}}\|f\|_{\mathrm{C}^{m, \alpha}}+\|g\|_{\mathrm{C}^{0}}\right) .
$$

Corollary 1.14. Under the assumptions from Lemma 1.13 we have that for a constant $C=C\left(m, \varrho, \varrho^{\prime}\right)$

$$
\|g \circ f\|_{\mathrm{C}^{m, \alpha}} \leq C\left(\|g\|_{\mathrm{C}^{m, \alpha}}\|f\|_{\mathrm{C}^{m, \alpha}}+\|g\|_{\mathrm{C}^{0}}\right) .
$$

Proof. Obvious consequence from Lemma 1.13

Corollary 1.15. Let $\varrho, \varrho^{\prime}>0, m \geq 1$ and $\alpha \in[0,1]$. For a function $f \in$ $\mathrm{C}^{m, \alpha}\left(\mathrm{B}_{\mathbb{R}^{d}}[0, \varrho], \mathbb{R}^{d^{\prime}}\right)$ with $f\left(\mathrm{~B}_{\mathbb{R}^{d}}[0, \varrho]\right) \subset \mathrm{B}_{\mathbb{R}^{d^{\prime}}}\left[0, \varrho^{\prime}\right]$ and a metric, i.e. matrix valued function, $g \in \mathrm{C}^{m-1, \alpha}\left(\mathrm{B}_{\mathbb{R}^{d^{\prime}}}\left[0, \varrho^{\prime}\right], \mathbb{R}^{d^{\prime 2}}\right)$ we have that for some constant $C=C\left(m, \varrho, \varrho^{\prime}, d\right)$ the pull-back is subject to the bound

$$
\left\|f^{*} g\right\|_{\mathrm{C}^{m-1, \alpha}} \leq C\left(\|g\|_{\mathrm{C}^{m-1, \alpha}}\|f\|_{\mathrm{C}^{m-1, \alpha}}+\|g\|_{\mathrm{C}^{0}}\right)\|f\|_{\mathrm{C}^{m, \alpha}}^{2} .
$$

Proof. Use Corollaries 1.11 and 1.14

$$
\begin{aligned}
\left\|\left(f^{*} g\right)_{\mu \nu}\right\|_{\mathrm{C}^{m-1, \alpha}} & =\left\|\sum_{\mu^{\prime} \nu^{\prime}}\left(g_{\mu^{\prime} \nu^{\prime}} \circ f\right) f_{\mu^{\prime}, \mu} f_{\nu^{\prime}, \nu}\right\|_{\mathrm{C}^{m-1, \alpha}} \\
& \leq \sum_{\mu^{\prime} \nu^{\prime}}\left\|\left(g_{\mu^{\prime} \nu^{\prime}} \circ f\right)\right\|_{\mathrm{C}^{m-1, \alpha}}\left\|f_{\mu^{\prime}, \mu}\right\|_{\mathrm{C}^{m-1, \alpha}}\left\|f_{\nu^{\prime}, \nu}\right\|_{\mathrm{C}^{m-1, \alpha}} \\
& \leq C^{\prime}\|(g \circ f)\|_{\mathrm{C}^{m-1, \alpha}}\|f\|_{\mathrm{C}^{m, \alpha}, \alpha}\|f\|_{\mathrm{C}^{m, \alpha}} \\
& \leq C\left(\|g\|_{\mathrm{C}^{m-1, \alpha}}\|f\|_{\mathrm{C}^{m-1, \alpha}}+\|g\|_{\mathrm{C}^{0}}\right)\|f\|_{\mathrm{C}^{m, \alpha}}^{2}
\end{aligned}
$$

where $f_{\kappa, \lambda}=\frac{\delta}{\delta x_{\lambda}} f_{\kappa}$. 
Lemma 1.16 ([CDK11, Theorem 16.32]). Let $\varrho, \varrho^{\prime}>0, m \geq 1$ an integer, and $\alpha \in[0,1]$. Let $c \geq 0$. Further let $f \in \mathrm{C}^{m, \alpha}\left(\mathrm{B}[0, \varrho], \mathbb{R}^{d}\right)$ and $g \in \mathrm{C}^{m, \alpha}\left(\mathrm{B}\left[0, \varrho^{\prime}\right], \mathbb{R}^{d}\right)$ with $f(\mathrm{~B}[0, \varrho]) \subset \mathrm{B}\left[0, \varrho^{\prime}\right]$ and $g\left(\mathrm{~B}\left[0, \varrho^{\prime}\right]\right) \subset \mathrm{B}[0, \varrho]$ such that

$$
g \circ f=i d \quad \text { and } \quad\|g\|_{\mathrm{C}^{1}},\|f\|_{\mathrm{C}^{1}} \leq c .
$$

Then there exists a constant $C=C\left(c, m, \varrho, \varrho^{\prime}\right)$ such that

$$
\|f\|_{\mathrm{C}^{m, \alpha}} \leq C\|g\|_{\mathrm{C}^{m, \alpha}} .
$$

Lemma 1.17 ([CDK11. Theorem 16.39]). Let $\varrho, \varrho^{\prime}>0, m \geq 0$ an integer, and $\alpha \in[0,1]$ with $m+\alpha \geq 1$. Let $c \geq 0$. Further let $u, v \in \mathrm{C}^{m, \alpha}\left(\mathrm{B}[0, \varrho], \mathbb{R}^{d}\right)$ and $g \in$ $\mathrm{C}^{m, \alpha}\left(\mathrm{B}\left[0, \varrho^{\prime}\right], \mathbb{R}^{d}\right)$ with $u(\mathrm{~B}[0, \varrho]), v(\mathrm{~B}[0, \varrho]) \subset \mathrm{B}\left[0, \varrho^{\prime}\right]$ and $g\left(\mathrm{~B}\left[0, \varrho^{\prime}\right]\right) \subset \mathrm{B}[0, \varrho]$ such that

$$
\|u\|_{\mathrm{C}^{1}},\|v\|_{\mathrm{C}^{1}} \leq c .
$$

Then there exists a constant $C=C\left(c, m, \varrho, \varrho^{\prime}\right)$ such that

$$
\|g \circ u-g \circ v\|_{\mathrm{C}^{m, \alpha}} \leq C\|g\|_{\mathrm{C}^{m, \alpha}}\left(1+\|u\|_{\mathrm{C}^{m, \alpha}}+\|v\|_{\mathrm{C}^{m, \alpha}}\right)\|u-v\|_{\mathrm{C}^{m, \alpha}} .
$$

Moreover, we conclude from the following:

Corollary 1.18. Let $\varrho, \varrho^{\prime}>0, m \geq 1$ an integer, and $\alpha \in[0,1], \varrho^{\prime \prime} \in(0, \varrho]$. Let $c \geq 0$. Let $f_{n}, f \in \mathrm{C}^{m, \alpha}\left(\mathrm{B}\left[0, \varrho^{\prime}\right], \mathbb{R}^{d}\right)$ with inverses $g_{n}, g \in \mathrm{C}^{m, \alpha}\left(\mathrm{B}[0, \varrho], \mathbb{R}^{d}\right)$ (i.e. $g_{n} \circ f_{n}=i d$ and $g \circ f=i d$ ) such that

$$
f_{n} \rightarrow f \quad \text { in } \mathrm{C}^{m, \alpha} \text {-norm }
$$

and $\left\|g_{n}\right\|_{\mathrm{C}^{1}},\|g\|_{\mathrm{C}^{1}},\left\|f_{n}\right\|_{\mathrm{C}^{1}},\|f\|_{\mathrm{C}^{1}} \leq c$.

Assume further that on $\mathrm{B}\left(0, \varrho^{\prime \prime}\right)$ the converse equalities $f_{n} \circ g_{n}=i d$ and $f \circ g=i d$ hold. Then the inverses $g_{n}$ converge to $g$ in $\mathrm{C}^{m, \alpha}-$ norm on $\mathrm{B}\left(0, \varrho^{\prime \prime}\right)$.

Proof. By abuse of notation we write $g_{n}$ and $g$ for the restrictions $\left.g_{n}\right|_{\mathrm{B}\left(0, \varrho^{\prime \prime}\right)}$ and $\left.g\right|_{\mathrm{B}\left(0, \varrho^{\prime \prime}\right)}$. We estimate

$$
\begin{aligned}
\| g_{n} & -g \|_{\mathrm{C}^{m, \alpha}} \\
& =\left\|\left(g_{n}-g\right) \circ f \circ g\right\|_{\mathrm{C}^{m, \alpha}} \\
& =\left\|g_{n} \circ f \circ g-g \circ f \circ g\right\|_{\mathrm{C}^{m, \alpha}} \\
& =\left\|g_{n} \circ f \circ g-g_{n} \circ f_{n} \circ g+g_{n} \circ f_{n} \circ g-g \circ f \circ g\right\|_{\mathrm{C}^{m, \alpha}}
\end{aligned}
$$

as $g_{n} \circ f_{n}=g \circ f=i d$

$$
=\left\|g_{n} \circ f \circ g-g_{n} \circ f_{n} \circ g\right\|_{\mathrm{C}^{m, \alpha}}
$$

apply Lemma 1.17 for $u=f \circ g$ and $v=f_{n} \circ g$

$$
\leq C\left\|g_{n}\right\|_{\mathrm{C}^{m, \alpha}}\left(1+\|f \circ g\|_{\mathrm{C}^{m, \alpha}}+\left\|f_{n} \circ g\right\|_{\mathrm{C}^{m, \alpha}}\right)\left\|\left(f-f_{n}\right) \circ g\right\|_{\mathrm{C}^{m, \alpha}} .
$$

The first factors are bounded by assumption. The last factor converges to 0 as $n \rightarrow \infty$ due to the composition estimate from Lemma 1.13 and the assumption that $f_{n} \rightarrow f$ in $\mathrm{C}^{m, \alpha}$-norm. 


\subsubsection{Metric tensors of low regularity}

The object we study in this thesis are Riemannian $d$-dimension manifolds (or $d$-manifolds)

$$
M=(M, g)
$$

where the tensor $g$ is not assumed to be smooth but of some lower regularity. To be precise by a $g$ we understand the following data:

- A covering collection $\left\{\varphi_{i}: V_{i} \rightarrow U_{i}\right\}_{i \in I}$ of charts on $M$ that is compatible with the continuous structure on $M$, i.e. $\varphi_{i}$ is continuous and $V_{i} \subset \mathbb{R}^{d}$ is open for each $i$.

- on each $V_{i}$ there is a Riemannian tensor given, i.e. a measurable map $g^{i}$ from $V_{i}$ into symmetric positive definite $(d \cdot d)$-matrices.

- each $g^{i}$ induces a metric in $V_{i}$ by

$$
|\xi \eta|_{i}:=\inf _{\gamma} \int_{[0,1]}(\nabla \gamma(t))^{\mathrm{T}} g^{i}(\nabla \gamma(t)) \mathrm{d} t
$$

where the infimum is taken over all $\mathrm{C}^{1}$-curves $\gamma:[0,1] \rightarrow V_{i}$ from $\xi$ to $\eta$ and $\nabla \gamma(t)$ denotes the gradient at $t$. The compatibility condition for the charts is only that for each $x \in M$ and indices $i, j$ such that $x \in U_{i} \cap U_{j}$ there is a neighborhood $U \subset U_{i} \cap U_{j}$ of $x$ such that the metrics $|. .|_{i}$ and $|.|_{j}$ agree on $U$, i.e.

$$
\left|\varphi_{i}^{-1}(x) \varphi_{i}^{-1}(y)\right|_{i}=\left|\varphi_{j}^{-1}(x) \varphi_{j}^{-1}(y)\right|_{j}
$$

for all $x, y \in U$.

In this thesis we will focus on Hölder regularity, though Sobolev regularity is also studied sporadically Heb96. Note that there is so far no analytic requirement on the regularity of the transition functions involved. We will see below how a smooth structure is fixed by a metric tensor of $\mathrm{C}^{\alpha}$-regularity for $\alpha>0$. In this direction first note a classical theorem by Whitney that a $\mathrm{C}^{1}$-atlas on a topological manifold uniquely determines a compatible smooth atlas Hir97. Theorem 2.9].

The following notion of convergence is also called Cheeger-Gromov convergence.

Definition 1.19 ( $\mathrm{C}^{m, \alpha}$-convergence). A sequence $\left(M_{n}\right)$ of pointed complete Riemannian manifolds $\mathrm{C}^{m, \alpha}$-converges to a pointed Riemannian manifold $M$ if the manifolds $M, M_{1}, M_{2}, \ldots$ admit a $\mathrm{C}^{1}$-atlas, the charts defining the respective Riemannian metrics belong to a respective $\mathrm{C}^{1}$-atlas, and for every $r>0$ there are a domain $\Omega \supset \mathrm{B}(p, r)$ in $M$ and (smooth with respect to the respective $\mathrm{C}^{1}$-structures) embeddings $\iota_{n}: \Omega_{n} \rightarrow M$ for large $n$ such that

$$
\begin{aligned}
\Omega_{n} & \supset \mathrm{B}\left(p_{n}, r\right), & \Omega \subset \iota\left(\Omega_{n}\right), \\
\iota_{n}\left(p_{n}\right) & =p, & \left(\iota_{n}^{-1}\right)^{*} g_{n} \stackrel{n \rightarrow \infty}{\longrightarrow} g \text { on } \Omega \text { in the } \mathrm{C}^{m, \alpha} \text {-sense, }
\end{aligned}
$$

where the last condition means that there are charts $\varphi_{s}: V_{s} \rightarrow U_{s} \subset M$ such that $\bigcup_{s} V_{s}=\Omega$ and $\left(\iota_{n}^{-1}\right)^{*} g_{n} \mathrm{C}^{m, \alpha}$-converges to $g$ on any chart $U_{s}$. 


\subsubsection{Chart norms}

In the next step we quantify the regularity of $\mathrm{C}^{m, \alpha}$-metrics. We follow Petersen [Pet16, § 11.3.1]. Another exposition is found in [Ron10. $\S$

Definition 1.20 (chart norm). Let $(M, p)$ be a pointed Riemannian $d$-manifold. The $\mathrm{C}^{m, \alpha}$-norm on the scale of $\varrho$ of $(M, p),\|(M, p)\|_{\mathrm{C} m, \alpha}^{\varrho}$, is the supremal real number such that for all $\Theta$ it holds that

$$
\|(M, p)\|_{\mathrm{C}^{m, \alpha}}^{\varrho} \leq \Theta
$$

where $\varrho$ is an upper index, whenever we find a chart (i.e. continuous map compatible with $g$ in the sense of $\S 1.3 .2$

$$
\varphi:\left(\mathrm{B}_{\mathbb{R}^{d}}(0, \varrho), 0\right) \rightarrow(U, p) \subset M
$$

such that for the (matrix valued) metric tensor $g_{\text {.. }}$ on $\mathrm{B}(0, \varrho)$ we have the following bounds

(i) $\|\mathrm{T} i d\|,\left\|\mathrm{T} i d^{-1}\right\| \leq e^{\Theta}$ for $i d:\left(\mathrm{B}(0, \varrho),\langle., .\rangle_{\text {Eucl. }}\right) \rightarrow\left(\mathrm{B}(0, \varrho), g_{. .}\right)$and $\mathrm{T} \varphi$, $\mathrm{T} \varphi^{-1}$ the differential maps between tangent bundles;

in case of a metric tensor $g$ defined on the tangent bundle of a smooth manifold this can be expressed in a coordinate free way by the bounds $\|\mathrm{T} \varphi\| \leq e^{\Theta}$ on $\left(\mathrm{B}(0, \varrho),\langle., .\rangle_{\text {Eucl. }}\right)$ and $\left\|\mathrm{T} \varphi^{-1}\right\| \leq e^{\Theta}$ on $\left(U,\left.g\right|_{U}\right) ;$

(ii) $\varrho^{|a|+\alpha}\left\|D^{a} g_{\text {.. }}\right\|_{\alpha} \leq \Theta$ on the Hölder semi-norm for all multi-indices $a$ with $0 \leq|a| \leq m$.

To refine this terminology, we say that a chart $\varphi: \mathrm{B}(0, \varrho) \rightarrow U \subset M$ has $\mathrm{C}^{m, \alpha}$-norm on the scale of $\varrho$ bounded by $\Theta$, or

$$
\|\varphi\|_{\mathrm{C}^{m, \alpha}}^{\varrho} \leq \Theta
$$

if both conditions above hold. If $\varrho^{\prime}<\varrho$, the norm $\|\varphi\|_{\mathrm{C}^{m, \alpha}}^{\varrho^{\prime}}$ is understood as the norm of the restriction of $\varphi$ to $\mathrm{B}\left(0, \varrho^{\prime}\right)$. Moreover, we say that a chart $\varphi$ is a chart at $x \in M$ if $\varphi(0)=x$. To globalize, we define

$$
\|M\|_{\mathrm{C}^{m, \alpha}}^{\varrho}:=\sup _{p \in M}\|(M, p)\|_{\mathrm{C}^{m, \alpha}}^{\varrho} .
$$

For $m=0,1, \ldots$ and $\alpha \in[0,1]$, let $\mathrm{P}_{\mathrm{C}^{m}, \alpha}^{d, \varrho} \leq \Theta$ denote the class of (isomorphisms classes of) pointed complete Riemannian $d$-manifolds with (global) $\mathrm{C}^{m, \alpha}$-norm on the scale of $\varrho$ bounded by $\Theta$.

From now on forward we will restrict to the case

$$
\alpha>0
$$

The reason to do so is first to exclude the case $m+\alpha=0$ in which a system of continuous coordinate charts with $\mathrm{C}^{0}$-bounded metric tensor does not fix a smooth structure. The other reason is that we are not able to apply Arzelà-Ascoli by lowering $\alpha$.

As Petersen Pet16. Proposition 11.3.2] states, the chart norm enjoys in case $\alpha>0$ the properties that

$$
\left\|\left(M, \lambda^{-2} g, p\right)\right\|_{\mathrm{C}^{m, \alpha}}^{\varrho}=\|(M, g, p)\|_{\mathrm{C}^{m, \alpha}}^{\lambda \varrho}
$$


for $\lambda>0$ and that

$$
\|(M, p)\|_{\mathrm{C}^{m, \alpha}}^{\varrho} \rightarrow 0
$$

as $\varrho$ goes to 0 . Moreover, given any chart $\varphi$ with $\|\varphi\|_{\mathrm{C}^{0}}^{\varrho} \leq \Theta$, we find that

$$
e^{-\Theta}|\xi| \leq|\varphi(\xi) \varphi(0)| \leq e^{\Theta}|\xi|
$$

Another variation of this concept is crucial to our application: harmonic coordinates. A chart $\varphi: \mathrm{B}(0, \varrho) \rightarrow M$ is harmonic if each component of the function $\varphi^{-1}: \varphi(\mathrm{B}(0, \varrho)) \rightarrow \mathbb{R}^{d}$ is harmonic, where harmonic means that the Laplace operator vanishes at this function. If the metric tensor admits at least one derivative, we are at liberty to consider the classical second-order Laplace operator

$$
\begin{aligned}
\Delta(u) & =\sum_{i j} g^{-1 / 2} \partial_{i}\left(\sqrt{g} g^{i j} \partial_{j} u\right) \\
& =\sum_{i j}\left(g^{i j} \partial_{j} \partial_{i}+\left(\partial_{i} g^{i j}+g^{-1}\left(\partial_{i} g\right) g^{i j}\right) \partial_{j}\right) u .
\end{aligned}
$$

But in order to formulate this in full generality also for the $m=0$ case we have to consider a weak version of the Laplace operator. Such a version is given by Taylor [Tay00, p. 140] in form of

$$
\Delta^{\mathrm{w}}(u)=\sum_{i j}\left(\sqrt{g} g^{i j} \partial_{j} u\right) \partial_{i}
$$

where $\Delta^{\mathrm{w}}(u)$ is treated as a distribution, i.e. a map $\varphi \mapsto \int \Delta^{\mathrm{w}}(u)(\varphi) \mathrm{d} x$ for $\varphi \in \mathrm{C}_{0}^{1}(\mathrm{~B}[0, \varrho], \mathbb{R})$, and the $g^{-1 / 2}$ coefficient gets absorbed by the measure, a trick that works fine if $\Delta^{\mathrm{w}}(u)$ is non-singular.

Based on these definitions and following Pet16, § 11.3.4], we define in parallel to $\|(M, p)\|_{\mathrm{C}^{m, \alpha}}^{\varrho},\|M\|_{\mathrm{C}^{m, \alpha}}^{\varrho}$, and $\mathrm{P}_{\mathrm{C}^{m, \alpha} \leq \Theta}^{d, \varrho}$, harmonic chart norms and regularity classes of manifolds with harmonic tensors

$$
\|(M, p)\|_{\mathrm{C}^{m, \alpha}}^{\varrho \text { har }}, \quad\|M\|_{\mathrm{C}^{m, \alpha}}^{\varrho \text { har }}, \quad \text { and } \quad \mathrm{P}_{\mathcal{M}^{m, \alpha} \leq \Theta}^{d, \varrho \text { har }}
$$

where the only difference is that all charts considered have to be harmonic. Note that without any regularity theory it is not obvious that these norms can have finite values and that the classes $\mathrm{P} \mathcal{M}_{\mathrm{C}^{m, \alpha}}^{d, \varrho} \leq \Theta$ har are non-empty.

\subsubsection{Regularity of transition maps}

It may seem conspicuous that the chart norm, as formulated in Definition 1.20 . takes the regularity of the metric tensor but not of transition functions into account. Actually, a geometric miracle is happening and regularity of the former implies the required regularity of the latter. In the harmonic case the regularity of transition functions is even one degree higher. The following theorem states these claims quantitatively:

Theorem 1.21. Let $m \in\{0,1,2, \ldots\}$ and $\alpha \in[0,1)$. Let $M$ be a topological $d$ manifold. Further let $\varphi_{i}: \mathrm{B}(0, \varrho) \rightarrow U_{i} \subset M, i=1,2$, be two coordinate patches. 
Let $V:=\varphi_{1}(\mathrm{~B}(0, \varrho)) \cap \varphi_{2}(\mathrm{~B}(0, \varrho))$. Assume that on $U_{1}$ and $U_{2}$, respectively, are endowed with a Riemannian tensor $g^{1}$ and $g^{2}$, respectively, such that

$$
\varphi:=\varphi_{2}^{-1} \circ \varphi_{1}: \varphi_{1}^{-1}(V) \rightarrow \varphi_{2}^{-1}(V)
$$

is a locally distance preserving homeomorphism with respect to $\left.g^{1}\right|_{\varphi_{1}^{-1}(V)}$ and $\left.g^{2}\right|_{\varphi_{2}^{-1}(V)}$, i.e. for every point $x \in \varphi_{2}^{-1}(V)$ there is a small neighborhood on which $\varphi$ is distance preserving. Then

(i) if $m+\alpha>0$, then $\varphi$ is of class $\mathrm{C}^{m+1, \alpha}$;

(ii) if $m+\alpha>0, \alpha>0$, and $\left\|\varphi_{i}\right\|_{\mathrm{C}^{m, \alpha}}^{\varrho} \leq \Theta$ for $i=1,2$, then for any $r>0$ there is a constant $C=C(d, \varrho, \Theta, m, \alpha, r)$

$$
\left\|\left.\varphi\right|_{\varphi^{-1}\left(V_{r}\right)}\right\|_{\mathrm{C}^{m+1, \alpha}} \leq C
$$

where $V_{r}:=\varphi_{1}(\mathrm{~B}(0, \varrho-r)) \cap \varphi_{2}(\mathrm{~B}(0, \varrho-r)) ;$

(iii) if in addition to the assumptions from (ii) $\varphi_{1}$ and $\varphi_{2}$ are harmonic, then $\varphi$ is even of class $\mathrm{C}^{m+2, \alpha}$ and for any $r>0$ the bound (1.24) holds with $m+1$ replaced by $m+2$ and $C$ a constant depending on the same parameters;

(iv) in the case $m=0$ and $\alpha=0$ the map $\varphi$ need not to be of class $\mathrm{C}^{1}$.

Proof. Part (i) is a result of Taylor [Tay06, Theorem 2.1]. Part (ii) is a refinement of Taylors' result, which we will explain below. First we state why $\varphi$ almost everywhere is locally bi-Lipschitz and subject to the equation $\left.\varphi^{*} g^{2}\right|_{\varphi_{2}^{-1}(V)}=$ $\left.g^{1}\right|_{\varphi_{1}^{-1}(V)}$ : Since both metrics are continuous, $\varphi$ is locally bi-Lipschitz with respect to Euclidean distance. Hence it is differentiable almost everywhere by Rademacher's theorem Fed14 § 3.1.6]. Moreover, since $\varphi$ maps Lipschitz curves to Lipschitz curves and preserves lenghts with respect to the metric tensors, the map $\mathrm{T}_{x} \varphi:\left(\mathbb{R}^{d},\left.g^{1}\right|_{x}\right) \rightarrow\left(\mathbb{R}^{d},\left.g^{2}\right|_{x}\right)$, where it exists, is a linear isometry by polarization identity. Therefore $\left(\left.g^{1}\right|_{x}\right)_{i j}=\sum_{\lambda, \mu=1}^{d}\left(\left.g^{2}\right|_{x}\right)_{\lambda \mu} \varphi_{\lambda, i} \varphi_{\mu, j}$.

Now we refine Taylor's argument. For the map $\varphi$ the proof shows that it is of class $\mathrm{C}^{m+1, \alpha}$. The proof goes by regularizing the coordinate system on a small ball $B$ around $\varphi(p) \in \varphi_{2}^{-1}(V)$ yielding some harmonic coordinates $u: B \rightarrow \mathbb{R}^{d}$. By linear transformation of coordinates we can assume that $B$ is centered at 0 . Using regularity theory, Taylor can show that not only $u$ and $\left.u \circ \varphi\right|_{\varphi^{-1} B}$ are of class $\mathrm{C}^{m+1, \alpha}$, but also $u^{-1}$. The desired regularity of $\left.\varphi\right|_{\varphi^{-1} B}$ follows then from the classes of regularity of $u^{-1}$ and $\left.u \circ \varphi\right|_{\varphi^{-1} B}$ by Lemma 1.13

First we will show that the ball can actually be chosen of fixed radius. The only thing to check is that the solution to the Dirichlet problem $\Delta^{\mathrm{w}} u=0$, $u_{\partial B}=i d$ actually gives a coordinate system, i.e. $u$ is injective. This is the case as soon as $\|i d-u\|_{\mathrm{C}^{1}}<1$ : Assume that $u(x)=u(y)$. By the mean value theorem the differential has to degenerate at a point on the line segment joining $x$ and $y$. This is in contradiction to $\|i d-u\|_{\mathrm{C}^{1}}<1$.

By a linear transformation bounded in terms of $\Theta$, we may actually assume that $g$. is Euclidean at $\varphi(p)$. The Laplacian of the vector difference $\Delta_{g}^{\mathrm{w}}(i d-u)=\Delta_{g}^{\mathrm{w}} i d=\left(\Delta_{g}^{\mathrm{w}}-\Delta_{\text {Eucl. }}^{\mathrm{w}}\right) i d$ is $\sum_{i j}\left(\sqrt{g} g^{i j}-\delta_{i j}\right) \partial_{j}$, that has to be understood as a distribution as explained right after (1.22). Standard theory GT77, Corollary 8.36] of the weak Dirichlet problem therefore gives the estimate

$$
\|i d-u\|_{\mathrm{C}^{1, \alpha}} \leq C\left(\|i d-u\|_{\mathrm{C}^{0}}+\left\|\left(\sum_{j} \sqrt{g} g^{i j}-\delta_{i j}\right)_{i=1}^{d}\right\|_{\mathrm{C}^{\alpha}}\right)
$$


where $C$ does only depend on $d$ and an upper bound on $\Theta$ (as by Definition 1.20 $\Theta$ controls the coefficients of $\Delta_{q}^{\mathrm{w}}$ ). The values of the function $u$ are bounded via the maximum principle GT77, Theorem 8.1] by the radius of $B$. Choosing $B$ sufficiently small, we can minimize the second summand. Since $g^{1 / 2} g^{i j}-\delta_{i j}$ vanishes at $\varphi(p)$ and by assumption $\varrho^{\alpha}\left\|g_{i j}\right\|_{\alpha} \leq \Theta$, Lemma 1.12 implies that there is a constant $C=C(d, \mathrm{~B}[0, \varrho-r], \Theta)=C(d, \varrho-r, \Theta)$ such that

$$
\left|\left(g^{1 / 2} g^{i j}-\delta_{i j}\right)(x)\right| \leq \varrho^{-\alpha} \cdot C \cdot\|g . .\|_{\mathrm{C}^{0, \alpha}\left(\mathrm{B}[0, r], \mathbb{R}^{d \cdot d}\right)} \cdot|x|^{\alpha} \leq C(|x| / \varrho)^{\alpha}
$$

for any $x \in B$. These two observation allow us to choose the required radius for $B$. Note that the assumption $\alpha>0$ is used here.

As the Euclidean metric is close to the intrinsic metric by 1.20 , the domain $\varphi_{1}^{-1}\left(V_{r}\right)$ has a positive distance $\tilde{r}=\tilde{r}(r, \Theta)$ in the intrinsic metric to the boundary of $V$. Assume that the radius of $B$ is smaller than $\tilde{r}$. This is to say that for every point $p$ in $\varphi_{1}^{-1}\left(V_{r}\right)$ we can choose a harmonic coordinate system $u$ on $B$ around $\varphi(p)$ that is contained in $\varphi_{1}^{-1}(V)$. We seek a regularity estimate for the restriction of the harmonic function $u \circ \varphi$ on $\varphi^{-1} B$ to $\varphi^{-1}\left(\frac{1}{2} B\right)$. This is given by Schauder estimates, also called elliptic estimates, for both versions - the weak version $\Delta^{\mathrm{w}} u \circ \varphi=0$ (see $(1.22)$ ) in case $m=0$, and the classical version $\Delta u \circ \varphi=0$ (see (1.21) in case $m>0$. In the former case we apply again GT77. Corollary 8.36] and in the latter [GT77, Problem 6.1] getting the bound

$$
\left\|\left.u \circ \varphi\right|_{\varphi^{-1}\left(\frac{1}{2} B\right)}\right\|_{\mathrm{C}^{m+1, \alpha}} \leq C\left\|\left.u \circ \varphi\right|_{\varphi^{-1} B}\right\|_{\mathrm{C}^{0}}
$$

where $C$ depends only on $d, \alpha, \Theta$, and a lower bound on $d_{\text {Eucl }}\left(\varphi^{-1}\left(\frac{1}{2} B\right), \partial B\right)$. The latter term and $\left\|\left.u \circ \varphi\right|_{\varphi^{-1} B}\right\|_{C^{0}}$ are again bounded in terms of $\Theta$ by the distance comparison statement 1.20 . The inverse $u^{-1}$ is bounded in terms of $C$ and $\operatorname{radius}(B)$ due to Lemma 1.16 This concludes the proof of part (ii),

The harmonic case, part (iii), uses only the classical regularity result, [GT77. Problem 6.1], and is by far easier: As in harmonic coordinates the Laplace operator has the shape $g^{i j} \partial_{i} \partial_{j}$, all coefficients are actually of class $\mathrm{C}^{m, \alpha}$ in the classical problem. Hence direct application of the theorem to $\varphi$ gives $\left\|\left.\varphi\right|_{\varphi_{1}^{-1}\left(V_{r}\right)}\right\|_{\mathrm{C}^{m+2, \alpha}} \leq C\|\varphi\|_{\mathrm{C}^{0}}$ where $C$ is bounded in terms of $d, \alpha, \Theta$, and $\left.d_{\text {Eucl }}\left(\varphi_{1}^{-1}\left(V_{r}\right)\right), \partial \mathrm{B}(0, \varrho)\right)$. As in part (ii) a distance comparison argument based on 1.20 concludes the proof.

Finally, a counterexample proving part (iv) is given in HW53.

Remark 1.22 (Myer-Steenrod theorem and regularity of geodesics). A theorem establishing the regularity of isometries, as [Tay06, Theorem 2.1] in the proof above, is also called Myer Steenrod's theorem, going back to the first such result claimed by Myers and Steenrod MS39 in their ground-breaking paper on the isometry groups of Riemannian manifolds. They claimed that any distancepreserving map of $\mathrm{C}^{1}$-metrics is a $\mathrm{C}^{1}$-isometry. The flaw in their argument is the employment of normal coordinates, for the existence of which one has to require at least $\mathrm{C}^{1,1}$-regularity of the metric tensor $\mathrm{KSS13}$, Chr91, Appendix F] - a problem we bypass by the introduction of strainers, see $\S 3.2 .1$

Later Calabi and Hartman $\mathrm{CH} 70$ claimed to prove $\mathrm{C}^{k+1, \alpha}$-regularity of isometries for $k+\alpha>0$. But their argument proves only $\mathrm{C}^{k+1, \alpha / 2}$-regularity. More precisely, Theorem 3.1 therein is flawed claiming that a geodesic in a $\mathrm{C}^{k, \alpha}$-metric is itself of class $\mathrm{C}^{k+1, \alpha}$. But actually the statement is false and their 
argument proves only uniform $\mathrm{C}^{k+1, \alpha / 2}$-regularity, as pointed out by Reshetnyak [Res78. See [MT16, § 7] for a historical overview.

The question of optimal regularity of geodesics in rough Riemannian metrics was answered in case $k=0$ by Lytchak and Yaman [LY06, Theorem 1.4] who prove $\mathrm{C}^{1, \alpha /(2-\alpha)}$-regularity and also give counterexamples showing that their result is optimal. They even provide results for Finsler metric in [LY06, Theorem 1.3]. For a self-contained proof of $\mathrm{C}^{k+1, \alpha / 2}$-regularity with $k>1$ in the Riemannian case see [Pet01].

There is the following addition for a converging sequence of metric tensors.

Lemma 1.23. Let $m \geq 0$. Given a uniformly $\mathrm{C}^{m+2, \alpha}$-bounded sequence of maps $\varphi_{n}: U \rightarrow \mathbb{R}^{d}$ for an open domain $U \subset \mathbb{R}^{d}$ together with Riemannian metrics $g^{n}$ on $U$ such that $\varphi_{n}$ is harmonic with respect to the metric $g^{n}$ and $g^{n}$ does $\mathrm{C}^{m, \alpha}$-converge to some harmonic $g$. If $\varphi_{n}$ converges in $\mathrm{C}^{0}$ to some harmonic function $\varphi: U \rightarrow \mathbb{R}^{d}$, then $\varphi_{n}$ converges in $\mathrm{C}^{m+2, \alpha}$.

Proof. By the same result as used for the harmonic case in the last proof GT77. Problem 6.1] we have

$$
\begin{aligned}
& \lim _{n \rightarrow \infty}\left\|\varphi_{n}-\varphi\right\|_{\mathrm{C}^{m+2, \alpha}} \\
& \leq C(\lim _{n \rightarrow \infty}\|\underbrace{\Delta_{g}\left(\varphi_{n}-\varphi\right)}_{=\Delta_{g} \varphi_{n}}\|_{\mathrm{C}^{m, \alpha}}+\underbrace{\| \varphi_{n}-\Delta_{g_{n}} \varphi_{n}}_{n \rightarrow \infty} \\
& \quad=C \lim _{n \rightarrow \infty}\left\|\left(\Delta_{g}-\Delta_{g^{n}}\right) \varphi_{n}\right\|_{\mathrm{C}^{m, \alpha}} \\
& \leq C C^{\prime} \sup _{n}\left\|\varphi_{n}\right\|_{\mathrm{C}^{m+2, \alpha}} \lim _{n \rightarrow \infty}\left\|g-g^{n}\right\|_{\mathrm{C}^{m, \alpha}} \\
& \quad=0
\end{aligned}
$$

where $C^{\prime}$ depends on $d$ and the uniform $\mathrm{C}^{m+2, \alpha}$-bound on $\varphi_{n}$.

\subsubsection{Fundamental Theorem of Convergence Theory}

We state a version of Theorem 11.3.6 in Petersen Pet16] that gives fundamental properties of $\mathrm{P}_{\mathrm{C}^{m, \alpha}, \varrho, \Theta}^{d}$ in terms of $\mathrm{GH}$-convergence and goes back to Cheeger and Colding [CC97, Theorem 7.2].

Theorem 1.24 (Fundamental Theorem of Convergence Theory). Let $0<\beta<$ $\alpha \leq 1, d \geq 2$, and $m \geq 0$. On $\mathrm{P}_{\mathrm{C}^{m, \alpha} \leq \Theta}^{d, \varrho}$ the pointed $m m$-topology and the $\mathrm{C}^{m, \beta}$ topology coincide. They are compact. Moreover the notion of Pmm-convergence (see \$1.2.1) and $\mathrm{C}^{m, \beta}$-convergence coincide. The same holds for $\mathrm{P} \mathcal{M}_{\mathrm{C}^{m, \alpha} \leq \Theta}^{d, \varrho}$ har

Proof. Petersen Pet16, Theorem 11.3.6] states that $\mathrm{P}_{\mathrm{C}^{m, \alpha} \leq \Theta}^{d, \varrho}$ is compact in the $\mathrm{C}^{m, \beta}$-topology. As $\mathrm{C}^{m, \beta}$-convergence implies Pmm-convergence, the map from $\mathrm{P}_{\mathrm{C}^{m}, \alpha \leq \Theta}^{d, \varrho}$ to $\mathrm{PM}$ is continuous. Restricting the codomain of this map to its image we get a bijective continuous map from a compact space into a Hausdorff space, but therefore it is also a homeomorphism [Sch75, § I.7.2 Satz 3].

In the harmonic case, by Pet16, Corollary 11.3.8] the class $\mathrm{P}_{\mathrm{C}^{m, \alpha} \leq \Theta}^{d, \varrho \text { har }}$ compact in the $\mathrm{C}^{m, \beta}$-topology. Since $\mathrm{P}_{\mathrm{C}^{m, \alpha} \leq \Theta}^{d, \varrho \text { har }}$ is contained in $\mathrm{P} \mathcal{M}_{\mathrm{C}^{m, \alpha} \leq \Theta}^{d, \varrho}$, this implies our claim on $\mathrm{P} \mathcal{M}_{\mathrm{C}^{m, \alpha} \leq \Theta}^{d, \varrho}$ har 


\subsubsection{Spaces of field spaces}

Recall from (1.17) that we only consider Riemannian manifolds $M$ with

$$
\|M\|_{\mathrm{C}^{\alpha}}^{\varrho} \leq \Theta
$$

for some $\alpha, \varrho, \Theta>0$. Recall further that in this case a $\mathrm{C}^{1}$-atlas is fixed by Theorem 1.21 and therefore also a smooth structure on $M$ by a mentioned result of Hirsch [Hir97, Theorem 2.9].

Definition 1.25 ( $\mathrm{C}^{m, \alpha}$-tensor). Let $m^{\prime}, k, l \geq 0, \alpha^{\prime} \in(0,1]$, and $M$ a Riemannian manifold (with $\|M\|_{\mathrm{C}^{\alpha}}^{\varrho} \leq \Theta$ ). A $\mathrm{C}^{m^{\prime}, \alpha^{\prime}}$-tensor of rang $(k, l)$ is a section $s$ in $\mathrm{T}^{k, l} M$ such that $M$ is covered by some charts compatible with the smooth structure on which the tensor is a $\mathrm{C}^{m^{\prime}, \alpha^{\prime}}$-function. Let $\mathrm{C}^{m^{\prime}, \alpha^{\prime}}\left(M, \mathrm{~T}^{k, l} M\right)$ be the set all $\mathrm{C}^{m^{\prime}, \alpha^{\prime}}$-tensors of rang $(k, l)$.

In this section we define a space of spaces $(M, g, p, s)$ where $(M, g, p)$ is a pointed Riemannian manifold and $s$ is a section in $\mathrm{C}^{m^{\prime}, \alpha^{\prime}}\left(M, \mathrm{~T}^{k, l} M\right)$. Such a space is called field space. For the space of sections $\mathrm{C}^{m^{\prime}, \alpha^{\prime}}\left(M, \mathrm{~T}^{k, l} M\right)$ over a Riemannian manifold $M$ we have at each point $p \in M$ the Riemannian norm on the fiber of $p$. When we want to take derivatives into account, we are faced with the fact that there is no immediate way to do this in a coordinate free fashion since the metric admits no derivatives and hence there is no covariant derivative - see Lessa Les14, Les15] for a development of approach in the smooth case.

The idea of the tensor norm is to look at the section $s$ on some coordinate patches that are regular with respect to a the $\mathrm{C}^{m, \alpha}$-norm of the metric tensor.

Definition 1.26. $\left[\mathrm{C}^{m^{\prime}, \alpha^{\prime}}-\mathrm{C}^{m, \alpha}-\Theta\right.$-norm on the scale of $\left.\varrho\right]$ For $\alpha \in(0,1], \beta \in$ $(0, \alpha)$, and $k, l$ non-negative integers. For a section $s \in \mathrm{C}^{m^{\prime}, \alpha^{\prime}}\left(M, \mathrm{~T}^{k, l} M\right)$ over a manifolds $M \in \mathrm{P} \mathcal{M}_{\mathrm{C}^{m}, \alpha}^{d, \varrho} \leq \Theta$ the $\mathrm{C}^{m^{\prime}, \alpha^{\prime}}-\mathrm{C}^{m, \alpha}-\Theta$-norm on the scale of $\varrho$ of $(M, p)$ is defined as

$$
\inf \left\{\left\|\varphi^{*} s\right\|_{\mathrm{C}^{m^{\prime}, \alpha^{\prime}}} \mid \varphi:\left(\mathrm{B}_{\mathbb{R}^{n}}(0, \varrho), 0\right) \rightarrow(U, p) \subset M \quad \text { with }\|\varphi\|_{\mathrm{C}^{m, \alpha}} \leq \Theta\right\} .
$$

The $\mathrm{C}^{m^{\prime}, \alpha^{\prime}}-\mathrm{C}^{m, \alpha}-\Theta$-norm on the scale of $\varrho$ of a non-pointed space is defined as the supremum of all norms of spaces $(M, x, s)$ for all $x \in M$, like for the metric tensor. Let

$$
\mathrm{T}_{\mathrm{C}^{m^{\prime}, \alpha^{\prime}} \leq \Theta^{\prime}}^{\mathrm{k}, \mathrm{P}} \mathcal{M}_{\mathrm{C}^{m}, \alpha}^{d, \varrho} \leq \Theta \quad \text { and } \quad \mathrm{T}_{\mathrm{C}^{m^{\prime}, \alpha^{\prime}} \leq \Theta^{\prime}}^{\mathrm{k}, \mathrm{P}} \mathcal{M}_{\mathrm{C}^{m, \alpha} \leq \Theta}^{d, \varrho \text { har }} \text {, resp. }
$$

be the space of all (equivalence classes of) pointed Riemannian manifolds with sections $(M, p, s)$ such that $\|M\|_{\mathrm{C}^{m, \alpha}}^{d, \varrho} \leq \Theta$ and $\|M\|_{\mathrm{C}^{m, \alpha}}^{d, \varrho \text { har }} \leq \Theta$, resp., and $(M, s)$ has $\mathrm{C}^{m^{\prime}, \alpha^{\prime}}-\mathrm{C}^{m, \alpha}-\Theta$-norm on the scale of $\varrho$ not greater than $\Theta^{\prime}$.

Note that the definition treats the norm of the section different from the norm of the tensor since there is no factor $\varrho^{m^{\prime}+\alpha^{\prime}}$. It is important to further note that a bound in the $\mathrm{C}^{m^{\prime}, \alpha^{\prime}}-\mathrm{C}^{m, \alpha_{-}} \Theta$-norm does not imply that the section $s$ is actually a tensor of class $\mathrm{C}^{m^{\prime}}$ because there is no control on the $\mathrm{C}^{m^{\prime}}$-regularity of transition functions if $m^{\prime}$ is to large. More precisely, according to Theorem 1.21 we have

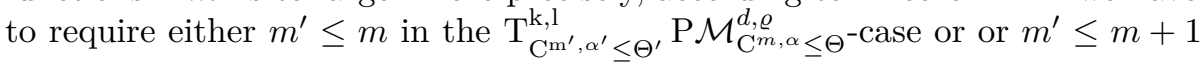
in the $\mathrm{T}_{\mathrm{C}^{\mathrm{m}^{\prime}, \alpha^{\prime}} \leq \Theta^{\prime}}^{\mathrm{k}, 1} \mathrm{P} \mathcal{M}_{\mathrm{C}^{m, \alpha} \leq \Theta^{- \text {case }}}^{d, \varrho \text { har }}$ 
We now prove a Fundamental Theorem of Convergence Theory for tensors, proved in appendix A.3

Lemma 1.27. Let $0 \leq \beta<\alpha$ and $0 \leq \beta^{\prime}<\alpha^{\prime}$ with $\beta^{\prime}<\alpha$. The space

$$
\mathcal{A}:=\mathrm{T}_{\mathrm{C}^{\mathrm{m}^{\prime}, \alpha^{\prime}} \leq \Theta^{\prime}}^{\mathrm{k}, \mathrm{P}} \mathcal{M}_{\mathrm{C}^{m, \alpha} \leq \Theta}^{d, \varrho}
$$

is completely metrizable and compact in the $\mathrm{C}^{m^{\prime}, \beta^{\prime}}-\mathrm{C}^{m, \beta}$-topology.

\subsubsection{Orientation and additional measures}

A manifold is oriented if there is a nowhere vanishing antisymmetric $d$-form on that manifold. Both equivalence classes of such $d$-forms are associated with an orientation.

Definition 1.28. Let $k, l \geq 0$. The space of oriented pointed Riemannian manifolds with a $(k, l)$-field is the topological space

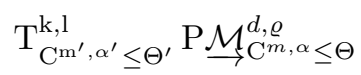

$$
\begin{aligned}
& :=\left\{\begin{array}{l|r}
(M, g, p, s \otimes \omega) \in \mathrm{T}_{\mathrm{C}^{m^{\prime}, \alpha^{\prime}} \leq \Theta^{\prime}}^{\mathrm{k}, \mathrm{P}} \mathrm{P}_{\mathrm{C}^{m}, \alpha}^{d, \varrho} \leq \Theta & \omega \text { antisymmetric, } \\
|\omega|_{g} \geq 1
\end{array}\right\} / \sim
\end{aligned}
$$

where $\sim$ is defined by $(M, g, p, s \otimes \omega) \sim\left(M^{\prime}, g^{\prime}, p^{\prime}, s^{\prime} \otimes \omega^{\prime}\right)$ if there is a diffeomorphism $f: M \rightarrow M^{\prime}$ such that

$$
\begin{aligned}
f(p) & =p^{\prime}, & g & =f^{*} g^{\prime} \\
f_{*} s & =s^{\prime}, & \left.f^{*}\left(\omega^{\prime}\right)\right|_{0} & =\left.\lambda \omega\right|_{0} \quad \text { for some } \lambda>0 .
\end{aligned}
$$

The space is given the quotient topology of the subspace topology of

$\left(\mathrm{T}_{\mathrm{C}^{m^{\prime}, \alpha^{\prime}} \leq \Theta^{\prime}}^{\mathrm{k}, \mathrm{P}}, \mathcal{M}_{\mathrm{C}^{m, \alpha} \leq \Theta}^{d, \varrho}\right.$, topology induced by $\mathrm{C}^{m^{\prime}, \alpha^{\prime}}-\mathrm{C}^{m, \alpha}$-convergence).

Note that by this construction a space with an orientation reversing symmetry is represented by only one point. Again we have a Fundamental theorem of Convergence Theory, whose proof is in the appendix:

Lemma 1.29. The space $\mathrm{T}_{\mathrm{C}^{m^{\prime}, \alpha^{\prime}} \leq \Theta^{\prime}}^{\mathrm{k}, \mathrm{P}} \stackrel{\mathcal{M}_{\mathrm{C}^{m, \alpha} \leq \Theta}^{d, \varrho}}{\underline{\varrho}}$ is completely metrizable and compact as a subspace of $\mathrm{T}_{\mathrm{C}^{m^{\prime}, \beta^{\prime}} \leq \Theta^{\prime}}^{\mathrm{k}, \mathrm{P}} \underset{\mathrm{M}^{m, \beta} \leq \Theta}{d, \varrho}$ for $\beta<\alpha$ and $\beta^{\prime}<\alpha^{\prime}$.

Definition 1.30. Let $\mathrm{T}_{\mathrm{C}^{m^{\prime}, \alpha^{\prime}} \leq \Theta^{\prime}}^{\mathrm{k}, 1} \mathrm{P}_{\mathrm{C}^{m}, \alpha \leq \Theta}^{d, \varrho\left[k^{\prime}, C\right]}, C>0$, denote the space of (equivalence classes of) spaces

$$
\left(M, g, p, s, \mu_{1}, \ldots, \mu_{k}\right)
$$

such that $(M, p, g, s) \in \mathrm{T}_{\mathrm{C}^{m^{\prime}, \alpha^{\prime}} \leq \Theta^{\prime}}^{\mathrm{k}, \mathrm{P}} \mathcal{M}_{\mathrm{C}^{m, \alpha} \leq \Theta}^{d, \varrho}$ and $\mu_{1}, \ldots, \mu_{k}$ are measure on $M$ satisfying the bound

$$
\mu_{i}(\mathrm{~B}[x, r]) \leq C^{r}+C
$$

for any $x \in M, r \geq 0$, and $i=1, \ldots, k$. On this space a notion of convergence is given by combination of the notion of convergence of $\mathrm{PM}\left[{ }^{[k+1]}\right.$ and tensor convergence, namely, $\left(M_{n}, p_{n}, g_{n}, s_{n}, \mu_{n 1}, \ldots, \mu_{n k}\right)$ converges to $\left(M, p, g, s, \mu_{1}, \ldots, \mu_{k}\right)$ 
if and only if for every radius $r$, index $i=1, \ldots, k$ (and sufficiently large $n$ ) there are a domain $\Omega \supset \mathrm{B}(p, r)$ and (smooth) embeddings

$$
\iota_{n}: \Omega_{n} \rightarrow M
$$

from domains $\Omega_{n} \supset \mathrm{B}\left(p_{n}, r\right)$ such that

- $\left(\iota_{n}^{-1}\right)^{*} g_{n}$ converges in $\mathrm{C}^{m, \alpha}$ to $g$ on $\Omega$,

- $\iota_{n *} s_{n}$ converges in $\mathrm{C}^{m^{\prime}, \alpha^{\prime}}$ to $s$ on $\Omega$, and

- $\iota_{n *}\left(b_{p_{n}, r} \mu_{n i}\right)$ weak ${ }^{\#}$ converges to $b_{p, r} \mu_{i}$ for $i=1, \ldots, k$ and $b_{., .}$as defined in 1.10 .

Likewise, define

$$
\mathrm{T}_{\mathrm{C}^{m^{\prime}, \alpha^{\prime}} \leq \Theta^{\prime}}^{\mathrm{k}, 1} \stackrel{\mathrm{P}}{\left.\mathcal{M}_{\mathrm{M}^{m, \alpha}}^{d, \varrho} \leq k^{\prime}, C\right]}
$$

as a quotient of a subset of $\mathrm{T}_{\mathrm{C}^{m^{\prime}, \alpha^{\prime}} \leq \Theta^{\prime}}^{\mathrm{k}, 1+\mathrm{d}} \mathrm{P}_{\mathrm{C}^{m, \alpha} \leq \Theta}^{d, \varrho\left[k^{\prime}, C\right]}$ as in Definition 1.28

Lemma 1.31. Lemma 1.27 holds for the spaces $\mathrm{T}_{\mathrm{C}^{m^{\prime}, \alpha^{\prime}} \leq \Theta^{\prime}}^{\mathrm{k}, 1} \mathrm{PM}_{\mathrm{C}^{m, \alpha} \leq \Theta}^{d, \varrho\left[k^{\prime}, C\right]}$ and

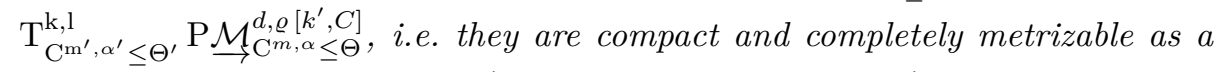

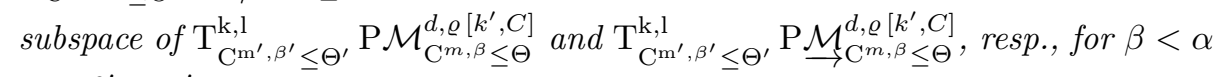
and $\beta^{\prime}<\alpha^{\prime}$.

The proof is found in the appendix. The spaces

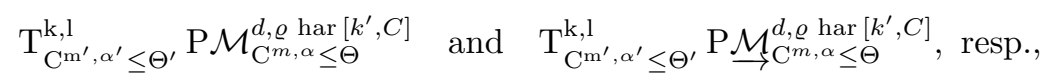

are defined as the preimage of the spaces

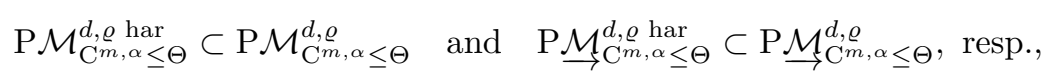

under the obvious forgetful map. Choosing some metric $d$ on

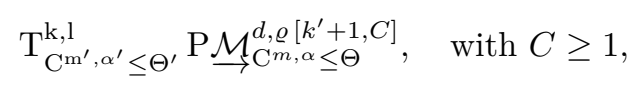

we can define a metric the space $\mathrm{T}_{\mathrm{C}^{m^{\prime}, \alpha^{\prime}} \leq \Theta^{\prime}}^{\mathrm{k}, \mathrm{PP}} \underset{\mathcal{M}_{\mathrm{C}^{m}, \alpha}^{d, \varrho} \leq \Theta}{\left[k^{\prime}, C\right]}$ on doubly pointed spaces $(M, g, p, q, s, \vec{\mu})$ with $\vec{\mu}=\mu_{1}, \ldots, \mu_{k^{\prime}}$ in parallel to the metric on doubly pointed mm-spaces from 1.14

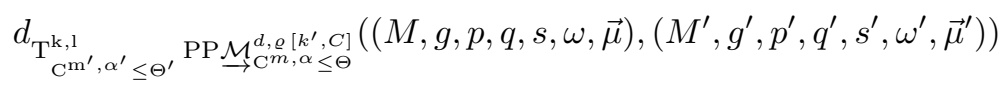

$$
\begin{aligned}
& :=|| p q|-| p^{\prime} q^{\prime}||+d\left(\left(M, g, p, s, \omega, \vec{\mu}, \delta_{q}\right),\left(M^{\prime}, g^{\prime}, p^{\prime}, s^{\prime}, \omega^{\prime}, \vec{\mu}^{\prime}, \delta_{q^{\prime}}\right)\right) \\
& +d\left(\left(M, g, q, s, \omega, \vec{\mu}, \delta_{p}\right),\left(M^{\prime}, g^{\prime}, q^{\prime}, s^{\prime}, \omega^{\prime}, \vec{\mu}^{\prime}, \delta_{p^{\prime}}\right)\right) .
\end{aligned}
$$




\section{Chapter 2}

\section{Main concepts and results}

The most general notion of a random space is a probability measure on the spaces $\mathrm{P} \mathcal{M}_{\mathrm{C}^{\alpha}<\Theta}^{d, \varrho}, \mathrm{P}_{\mathrm{C}^{\alpha}<\Theta}^{d, \varrho}$, or PM. But it is more suitable to restrict to a narrower class by imposing some condition, such as unimodularity, a way to formulate the independence of a random space and its base point. As special case of unimodular spaces are spaces that arise as limits of (deterministic) spaces viewed as random spaces.

\section{$2.1 \quad$ Random spaces}

As focus of this thesis is on oriented Riemannian manifolds we will state all definition for $\mathrm{P} \mathcal{M}_{\mathrm{C}^{\alpha} \leq \Theta}^{d, \varrho}$, though they work for $\mathrm{P}_{\mathcal{M}^{\alpha} \leq \Theta}^{d, \varrho}, \mathrm{PM}$, or other classes of spaces as well. The term law denotes in general a probability measure on a metric space, e.g. the distribution of a random variable with values in this space.

\subsubsection{Benjamini-Schramm limits}

The following definition was actually already formulated for PM in the introduction by Definition 0.1 .

Definition 2.1. Let $\varrho, \Theta>0$ and $\alpha \in(0,1]$. Let $\underset{\mathcal{M}}{\rightarrow}=\underline{\mathcal{M}}_{\mathrm{C}^{\alpha} \leq \Theta}^{d, \varrho}$ denote the class (of equivalence classes) of oriented compact Riemannian manifolds with $\mathrm{C}^{\alpha}$-norm on the scale of $\varrho$ not greater than $\Theta$. For any $M=(M$, vol $) \in \underline{M}$ let $\mu_{M}: M \rightarrow \mathrm{P} \mathcal{M}_{\mathrm{C}^{\alpha}<\Theta}^{d, \varrho}$ be the map $p \mapsto(M, p, v o l)$ that assigns to each point a pointed version of $M$. Further let $\tilde{\mu}$ be the map

$$
\tilde{\mu}: \underline{\mathcal{M}} \rightarrow \mathcal{P} P{ }^{P} \underline{\mathcal{M}}_{\mathrm{C}^{\alpha} \leq \Theta}^{d, \varrho}, \quad M \mapsto\left(\mu_{M}\right)_{*}\left(\frac{\text { vol }}{\operatorname{vol} M}\right)
$$

where vol is finite since $M$ is compact. We endow $\mathcal{M}$ with the topology induced by $\tilde{\mu}$. Likewise we say that a sequence $\left(M_{n}\right) \subset \underset{\mathcal{M}}{\longrightarrow}$ of spaces BS-converges if the laws $\tilde{\mu}(M)$ converge (against continuous test functions).

\subsubsection{Unimodular spaces}

Any random space in the in the closure of the image of $\tilde{\mu}$, as considered above, is unimodular. Further, all constructions we will do will have to satisfy this notion of 
independence. We will introduce the concept following Bow15. Unimodularity is again a convenient concept for any kind of random object to which BenjaminiSchramm convergence is applicable. The broadest study is again found in graph theory, e.g. Bor16] and [AL07] (see also Remark 2.5. But the concept is also studied, for example, for Riemannian manifolds with respect to smooth convergence [AB16.

Definition 2.2. A law $P$ on $P \mathcal{M}_{\mathrm{C}^{\alpha}}^{d, \varrho \Theta}$ is called unimodular if the Intrinsic Mass-Transport Principle holds, i.e. the left and right measures, $m_{\mathrm{L}}(P)$ and $m_{\mathrm{R}}(P)$, on the space of doubly pointed spaces $\mathrm{PP} \mathcal{M}_{\mathrm{C}^{\alpha} \leq \Theta}^{d, \varrho}$ induced by Lemma 1.7 (which is applicable due to part (i) of Lemma 2.3 below) and the maps from

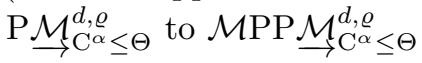

$$
\int f \mathrm{~d} \tilde{\mu}_{(.)}^{\mathrm{L}}:(M, p, v o l) \mapsto\left(\mu_{M}^{\mathrm{L}}\right)_{*} v o l \quad \text { and } \quad \tilde{\mu}_{(.)}^{\mathrm{R}}:(M, p, v o l) \mapsto\left(\mu_{M}^{\mathrm{R}}\right)_{*} v o l,
$$

where

$$
\mu^{\mathrm{L}}: x \mapsto(M, p, x) \quad \text { and } \quad \mu^{\mathrm{R}}: x \mapsto(M, x, p),
$$

are equal.

It is routine to check the following lemma:

Lemma 2.3. (i) the maps $\tilde{\mu}^{\mathrm{L}}$ and $\tilde{\mu}^{\mathrm{R}}$ are continuous;

(ii) for any bounded subset $A$ of $\mathrm{PPM}$ there is a uniform bound on $\tilde{\mu}_{M}^{\mathrm{L}}(A)$ and

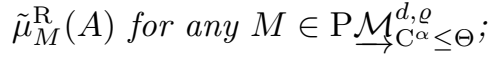

(iii) the maps $m_{\mathrm{L}}$ and $m_{\mathrm{R}}$ are continuous;

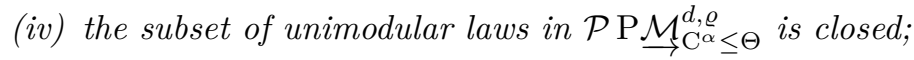

(v) the subset of unimodular laws in $\mathcal{P} \mathrm{P} \underline{\mathcal{M}}_{\mathrm{C}^{\alpha} \leq \Theta}^{d, \varrho}$ comprises $\tilde{\mu}(\underline{\mathcal{M}})$ (notation as in Definition 2.1.

Proof. For (i) we check the definition from (1.8) by taking a bounded continuous test function $f: \mathcal{M P P} \mathcal{M}_{\mathrm{C}^{\alpha} \leq \Theta}^{d, \varrho} \rightarrow \mathbb{R}$ with bounded support. Note that due to the term " ||$p q|-| p^{\prime} q^{\prime}|| "$ in definition 1.14 the distance of both base points of a space in the support of $f$ is bounded by some $l$. Observe that for a converging sequence $\left(M_{n}, p_{n}\right) \rightarrow(M, p)$ in $\mathcal{M P} \mathcal{M}_{\mathrm{C}^{\alpha} \leq \Theta}^{d, \varrho}$ (according to Definition 1.19 we have

$$
\begin{aligned}
\int f \mathrm{~d} \tilde{\mu}_{\left(M_{n}, p_{n}\right)}^{\mathrm{L}} & =\int f \mathrm{~d}\left(\mu_{\left(M_{n}, p_{n}\right)}^{\mathrm{L}}\right)_{*} v o l_{n}=\int f\left(\left(M_{n}, p_{n}, x\right)\right) \mathrm{d} v o l_{n}(x) \\
& =\int g_{n}(x) \mathrm{d} v o l_{n}(x)
\end{aligned}
$$

for $g_{n}: x \mapsto f\left(\left(M_{n}, p_{n}, x\right)\right)$. Likewise we have $\int f \mathrm{~d} \tilde{\mu}_{(M, p)}^{\mathrm{L}}=\int g \mathrm{~d} v o l$ for $g_{n}: x \mapsto$ $f((M, p, x))$. Note that $g_{n}$ is bounded, because $f$ is bounded, and moreover has a bounded support with diameter $\leq 2 l$ because for any two points $x, y$ we have that $g(x), g(y)>0$ implies $\left|p_{n} x\right|,\left|p_{n} y\right| \leq l$ and hence $|x y| \leq 2 l$. But convergence $\left(M_{n}, p_{n}\right) \rightarrow(M, p)$ according to Definition 1.19 implies that for 
sufficiently large $n$ there are invertible comparison maps $\iota_{n}: \Omega_{n} \subset M_{n} \rightarrow M$ such that the support of $g_{n}$ ( $g$, resp.) is contained in every $\Omega_{n}$ (in $\Omega$, resp.) and $\iota_{n \star}$ vol $_{n}$ converges to vol. Hence we have that

$\int g_{n} \mathrm{~d} v o l_{n}=\int g \circ \iota_{n}^{-1} \mathrm{~d} \iota_{n \star} \operatorname{vol}_{n}=\int g \circ \iota_{n}^{-1} \mathrm{~d}\left(\iota_{n \star} v o l_{n}-v o l\right)+\int g \circ \iota_{n}^{-1} \mathrm{~d} v o l$

Since the supports of each $g_{n}$ and $g$ have diameter $\leq 2 l$, there is a function $\chi: M \rightarrow[0,1]$ with bounded support which is 1 one every point which is in one of the supports of one of the $g_{n}$ 's or of $g$. Let $C$ be the bound of the absolute value of $f$. Hence the first summand $\int g \circ \iota_{n}^{-1} \mathrm{~d}\left(\iota_{n \star} v o l_{n}-v o l\right)$ is bounded in absolute value by $\int C \chi \mathrm{d}\left(\iota_{n \star} v o l_{n}-v o l\right)$ which converges to 0 by definition from (1.8).

The integrand of the second summand converges pointwise to $\int g \mathrm{~d} v o l$ since $M_{n}$ converges to $M$ via the $\iota_{n}$ 's. Hence it converges to $\int g \mathrm{~d} v o l$ by the dominated convergence theorem. This is to say that $\int f \mathrm{~d} \tilde{\mu}_{\left(M_{n}, p_{n}\right)}^{\mathrm{L}}$ converges to $\int f \mathrm{~d} \tilde{\mu}_{(M, p)}^{\mathrm{L}}$; thus $\tilde{\mu}^{\mathrm{L}}$ is continuous. The argument for $\tilde{\mu}^{\mathrm{R}}$ is the same.

For (ii) take a bounded set $A$ with diameter (see (1.1)) bounded by $l$. It is immediate from the term " ||$p q|-| p^{\prime} q^{\prime}||$ " in definition 1.14 of the distance in PPM that the distance of both base points of a space in $A$ is bounded by $l$. Moreover the volume $\mathrm{B}[p, l]$ has a uniform bound $v$ for any $(M, p, v o l) \in$ $\mathrm{P} \underline{\mathcal{M}}_{\mathrm{C}^{\alpha} \leq \Theta}^{d, \varrho}$ since otherwise there were a limit manifold of a sequence of manifolds $\left(M_{n}, p_{n}, \operatorname{vol}_{n}\right) \in \mathrm{P} \underline{\mathcal{M}}_{\mathrm{C}^{\alpha} \leq \Theta}^{d, \varrho}$ with $\operatorname{vol}_{n} \mathrm{~B}\left[p_{n}, l\right] \stackrel{n \rightarrow \infty}{\longrightarrow} \infty$. Hence we can estimate $\tilde{\mu}^{\mathrm{L}(M, p, v o l)}(A) \leq v \operatorname{vol\mathrm {B}}[p, l] \leq v$. By the same argument the values of $\tilde{\mu}^{\mathrm{R}}$ are bounded on bounded sets.

The claim (iii) follows directly from (iii) and Lemma 1.7. Claim (iv) follows from the fact that the unimodular laws are exactly the elements of the equalizer of $m_{\mathrm{L}}$ and $m_{\mathrm{R}}$, and this equalizer is closed because it is nothing but the preimage of the diagonal $\delta \subset\left(\operatorname{PP} \underset{\mathcal{M}_{\mathrm{C}^{\alpha}}^{d, \varrho}}{d,}\right)^{2}$ under the map $\left(m_{\mathrm{L}}, m_{\mathrm{R}}\right)$ and $\delta$ is closed because $\mathrm{PP} \underset{\mathcal{M}_{\mathrm{C}}^{\alpha} \leq \Theta}{d, \varrho}$ is Hausdorff.

For the last claim $(\mathrm{v})$ be calculate using the definition from Lemma 1.7

$$
\begin{aligned}
m_{\mathrm{L}}(\tilde{\mu}(M)) & =m_{\mathrm{L}}\left(\left(\mu_{M}\right)_{*} \frac{\operatorname{vol}}{\operatorname{vol} M}\right) \\
& =A \mapsto \int\left(\left(\mu_{\left(M^{\prime}, p^{\prime}\right)}^{\mathrm{L}}\right)_{*} \operatorname{vol}\right)(A) \mathrm{d}\left(\mu_{M}\right)_{*} \frac{\operatorname{vol}}{\operatorname{vol} M}\left(M^{\prime}, p^{\prime}\right) \\
& =A \mapsto \frac{1}{\operatorname{vol} M} \int_{M}\left(\left(\mu_{(M, p)}^{\mathrm{L}}\right)_{*} \operatorname{vol}\right)(A) \mathrm{d} \operatorname{vol}(p) \\
& =A \mapsto \frac{1}{\operatorname{vol} M} \iint \chi_{A}((M, p, q)) \mathrm{d} \operatorname{vol}(q) \mathrm{d} \operatorname{vol}(p) .
\end{aligned}
$$

By similar calculation we have that

$$
m_{\mathrm{R}}(\tilde{\mu}(M)): A \mapsto \frac{1}{\operatorname{vol} M} \iint \chi_{A}((M, q, p)) \mathrm{d} \operatorname{vol}(q) \mathrm{d} \operatorname{vol}(p) .
$$

Hence the equality $m_{\mathrm{L}}(\tilde{\mu}(M))=m_{\mathrm{R}}(\tilde{\mu}(M))$ is implied by Tonelli's theorem.

The term Mass-Transport Principle is motivated by the equality $\int f \mathrm{~d} m_{\mathrm{L}}=$ $\int f \mathrm{~d} m_{\mathrm{R}}$ for any Borel function $f: \mathrm{PPM} \rightarrow \mathbb{R}_{\geq 0}$ and by thinking of $f$ as the mass transported from $p$ to $q$. 
As mentioned above Definition 2.2 generalizes immediately to the space PM but also to the space

$$
\mathrm{T}_{\mathrm{C}^{m^{\prime}, \alpha^{\prime}} \leq \Theta^{\prime}}^{\mathrm{k},} \stackrel{\mathrm{P}}{\mathcal{M}_{\mathrm{C}^{m, \alpha} \leq \Theta}^{d, \varrho}\left[k^{\prime}+1, C\right]}, \quad \text { with } C \geq 1
$$

by replacing $\mathrm{PP} \underline{\mathcal{M}}_{\mathrm{C}^{\alpha} \leq \Theta}^{d, \varrho}$ with $\mathrm{T}_{\mathrm{C}^{m^{\prime}, \alpha^{\prime}} \leq \Theta}^{\mathrm{k}, 1}, \mathrm{PP} \underline{\mathcal{M}}_{\mathrm{C}^{m, \alpha} \leq \Theta}^{d, \varrho\left[k^{\prime}+1, C\right]}$, those metric is defined in 1.25 .

The term unimodular corresponds to the concept of unimodular groups in topological group theory. Let $G$ be a locally compact topological group and let $m_{\mathrm{L}}$ and $m_{\mathrm{R}}$ be the left and right Haar measures. Recall that $G$ unimodular if

- $m_{\mathrm{L}}$ and $m_{\mathrm{R}}$ coincide or, equivalently,

- the modular function $m$ on $G$ characterized by $m_{\mathrm{L}}(A g)=m(g) m_{\mathrm{L}}(A)$ is constant to 1 .

The following remarks make the connection to unimodular random spaces explicit.

Remark 2.4 (connection to Lie groups). We say that a sequence $M_{n}$ of pointed smooth Riemannian manifolds smoothly converges to a pointed smooth Riemannian manifolds $M$ if it converges in the sense of Definition 1.19 with $\mathrm{C}^{m, \alpha}$ replaced by $\mathrm{C}^{\infty}$. Definition 2.2 can be rephrased for the space of smooth Riemannian manifolds with the topology induced by smooth convergence.

For a (non-pointed) smooth Riemannian manifold $M$ with transitive isometry group the spaces $(M, p)$ and $(M, q)$ are isometric as pointed spaces for any choice of $p, q \in M$. Hence there is a unique way two view $M$ as a Dirac measure $\delta_{(M, p)}$ of the space of pointed smooth Riemannian manifolds. Abert and Biringer AB16 proved that for a smooth Riemannian manifold $M$ with transitive isometry group, the random space $\delta_{(M, p)}$ is unimodular if and only if the isometry group of $M$ is unimodular.

Remark 2.5 (connection to Cayley graphs). We explain how the term unimodular origins from graph theory and group theory, following AL07, § 3] and Ben+99. $\S 3]$. Consider the following definitions

- a (deterministic) locally finite graph $X$ is called unimodular if the group Aut $X$ endowed with the topology of pointwise convergence is unimodular;

- a (deterministic) locally finite graph $X$ is called unimodular with respect to a closed subgroup $G<$ Aut $X$ if $G$ is unimodular;

- a random graph $P \in \mathcal{P}$ (\{pointed graphs $\})$ is unimodular if the functions

$$
\mu^{\mathrm{L}}, \mu^{\mathrm{R}}: \mathcal{P}(\{\text { pointed graphs }\}) \rightarrow \mathcal{M} \mathcal{P}(\{\text { doubly pointed graphs }\})
$$

defined in parallel to Definition 2.2 are equal — for a suitable topology chosen on \{pointed graphs\}, see references mentioned above;

where the requirements of local finiteness of $X$ and closedness of $G$ guarantees local compactness. A crucial and direct combinatorial consequence of unimodularity of a graph $X$ with respect to some $G<$ Aut $X$ is that if $g y=x$ for some $x, y \in X$ and $g \in G$, then $\left|\operatorname{Stab}_{G} x\right|=\left|\operatorname{Stab}_{G} y\right|$ where |.| is the Haar measure and $\operatorname{Stab}_{G}(x)=\{g \in G \mid g x=x\}$ is the stabilizer of $x$. 
Aldous and Lyons AL07, Theorem 3.1] state that a random graph $P$ supported on a fixed (possibly infinite) graph ${ }^{1} X$ is unimodular if and only if $X$ is a (deterministic) unimodular graph and $c:=\sum_{x}\left|\operatorname{Stab}_{\text {Aut } X} x\right|^{-1}<\infty$ where $x$ ranges over a complete orbit section. This probability measure $P$ is unique and satisfies

$$
P((X, p))=\frac{1}{c\left|\operatorname{Stab}_{\text {Aut } X} p\right|} .
$$

By AL07, Remark 3.3] the same holds for Cayley graphs with respect to $G<$ Aut Cayley $(G)$ - or, if we formulate all definitions above for labeled graphs and mark the edges of Cayley $(G)$ by generators, $G$ itself is the automorphism group of Caley $(G)$ and the statement holds equivalently. This establishes the connection of all definition considered.

\subsubsection{Parameters and Testability}

By a parameter we will formally understand a partially defined real valued function on $\underset{\mathcal{M}_{\varphi}}{\longrightarrow} \subset \mathcal{P} P \stackrel{\mathcal{M}}{\rightarrow}_{\mathrm{C}^{\alpha} \leq \Theta}^{d, \varrho}$ (or $\mathbb{M}_{\varphi} \subset \mathcal{P} \mathrm{PM}$, alternatively).

Definition 2.6. A parameter is testable if it is continue and can be continued to the boundary of its domain.

Following Hungarian terminology, we define:

Definition 2.7. A testable parameter is testable in constant time if its domain is relatively compact.

In general statistical terminology one would rather speak of "estimating" than of "testing". The explanation for using statistical terminology will be given in the next Subsection in Theorem 2.10. For a better understanding of the latter definition in terms of measure theory recall from [Dud02, Theorem 11.5.4]

Lemma 2.8. A family of laws on a complete separable metric space $X$ is relatively compact if and only if it is uniformly tight, i.e. for every $\varepsilon>0$ there is a compact subset $X_{\varepsilon} \subset X$ such that $X_{\varepsilon}$ has measure greater than $1-\varepsilon$ with respect to any element of the family of laws.

Remark 2.9. Testability in a concept widely studied in graph theory. In this area, a tester for a property $P$ is a random algorithm that make a limited amount of queries and return with some probabilty $1-\varepsilon$ if a graph is $\varepsilon$-close (with respect to some distance) to some other graph with property $P$. An example for $P$ would be being 4-colorable and a typical metric for measuring closeness is the edit distance. See [Gol10 for an overview. There is also a concept of estimability, that is a bit closer to the characterization of testability in constant time we will obtain in Theorem 2.10 FN05.

\subsubsection{Characterization by random samples}

More crucial is the geometric motivation of this definition by Theorem 2.10 . which we are going to proof now. Define the sample function on P $\underline{\mathcal{M}}$ and PM by

$$
\begin{array}{rlrl}
s_{r}: & (M, g, \omega, p) & \mapsto\left(\mathrm{B}[p, r],\left.g\right|_{\mathrm{B}[p, r] \times \mathrm{B}[p, r]},\left.\omega\right|_{\mathrm{B}[p, r]}, p\right) \\
s_{r}: & (M, d, v o l, p) & \mapsto\left(\mathrm{B}[p, r],\left.d\right|_{\mathrm{B}[p, r] \times \mathrm{B}[p, r]},\left.v o l\right|_{\mathrm{B}[p, r]}, p\right) . &
\end{array} \quad \text { and }
$$

\footnotetext{
$1_{\text {i.e. }} P(\{(X, x) \mid x \in X\})=1$.
} 
We formulate and prove the following theorem in the mm-space case. The case of $\underline{\mathcal{M}}$ is parallel.

Theorem 2.10. Let $\varphi$ be a parameter that is testable in constant time. For any $\varepsilon>0$ there is a radius $r$ and a natural number $n$ and a tester $\tau$, i.e. a map $\tau:\left(s_{r}\left(\mathrm{PM}_{\varphi}\right)\right)^{n} \rightarrow \mathbb{R}$, such that the bound

$$
\operatorname{Prob}\left(\left|\varphi(M)-\tau\left(s_{r} X_{1}, \ldots, s_{r} X_{n}\right)\right|<\varepsilon\right)>1-\varepsilon \quad \text { for all }(M, d, v o l) \in \mathbb{M}_{\varphi}
$$

holds, where $X_{1}, \ldots, X_{n}$ are uncorrelated random variables with law $\frac{1}{\operatorname{vol}(M)}$ vol.

The proof is actually an application of the law of the large numbers as it uses the Stone-Weierstraß theorem which can be obtained from the latter.

Lemma 2.11. The assignments

$$
\begin{array}{ll}
\mathfrak{M} \mapsto \mathrm{C}^{0,1}(\mathfrak{M}):=\left(\mathrm{C}^{0,1}(\mathfrak{M}, \mathbb{R}),\|\cdot\|_{\mathrm{C}^{0}}\right) & \text { and } \\
\mathfrak{M} \mapsto \mathrm{C}^{0}(\mathcal{P} \mathfrak{M}):=\left(\mathrm{C}^{0}(\mathcal{P} \mathfrak{M}, \mathbb{R}),\|\cdot\|_{\mathrm{C}^{0}}\right) &
\end{array}
$$

constitute contravariant functors from Lip to Norm, where Lip is the category of metric spaces and bounded Lipschitz maps of Lipschitz constant at most 1 and Norm is the category of normed spaces and bounded linear maps. There is a natural transformation

$$
\eta_{\mathfrak{M}}:\left(\mathrm{C}^{0,1}(\mathfrak{M}, \mathbb{R}),\|\cdot\|_{\mathrm{C}^{0}}\right) \rightarrow\left(\mathrm{C}^{0}(\mathcal{P} \mathfrak{M}, \mathbb{R}),\|\cdot\|_{\mathrm{C}^{0}}\right)
$$

among these functors given by

$$
h \mapsto\left(P \mapsto \int h \mathrm{~d} P\right) .
$$

Proof. On morphisms both functors are defined by concatenation. Since the hom-sets of Lip are restricted to bounded Lipschitz maps, this assignment is well-defined for the functor $\mathrm{C}^{0,1}($.$) . The functor \mathrm{C}^{0}(\mathcal{P} ., \mathbb{R})$ is the concatenation of the covariant functor $\mathcal{P}$ and the contravariant functor $\mathrm{C}^{0}(., \mathbb{R})$. Both are even definable for arbitrary continuous functions.

As for the natural transformation map $\eta_{\mathfrak{M}}$ we immediately see that it is linear in $h$. Moreover given a function $h \in \mathrm{C}^{0,1}(\mathfrak{M})$ with $\|h\|_{\mathrm{C}^{0}} \leq 1$ we get that $\left\|P \mapsto \int h \mathrm{~d} P\right\|_{\mathrm{C}^{0}} \leq \sup _{x \in \mathfrak{M}} h(x) \leq 1$. Hence $\eta_{\mathfrak{M}}$ is bounded. To check that $\eta_{\mathfrak{M}}$ is natural observe for any Lipschitz map $f: \mathfrak{M} \rightarrow \mathfrak{N}$ and any $h \in \mathrm{C}^{0,1}(\mathfrak{M})$ we have $\eta_{\mathfrak{M}} \circ\left(\mathrm{C}^{0,1}(f)\right)(h)=\left(P \mapsto \int h \circ f \mathrm{~d} P\right)=\left(P \mapsto \int h \mathrm{~d} f_{*} P\right)=\left(\mathrm{C}^{0}(\mathcal{P} f)\right) \circ \eta_{\mathfrak{N}}(h)$.

Note that this lemma is related to Riesz representation theorem which states that if we restrict Lip to compact spaces, the natural transformation becomes an isomorphism.

Proof of Theorem 2.10. For convenience of notation define

$$
B_{r}^{n}:=\left(s_{r} X_{1}, \ldots, s_{r} X_{n}\right) \quad \text { and } \quad P_{M}:=\left(\mu_{M}\right)_{*}\left(\frac{\operatorname{vol}}{\operatorname{vol}(M)}\right) .
$$

Let $\mathrm{C}^{0}(),. \mathrm{C}^{0,1}($.$) be the functors defined in Lemma 2.11$ and let $\eta_{(.)}$be the natural transformation from $\mathrm{C}^{0}($.$) to \mathrm{C}^{0,1}($.$) defined therein.$ 
For a compact subset $\mathfrak{K} \subset \mathcal{P} \mathfrak{M}$, the algebra generated by the image of $\eta_{\mathfrak{M}}$ shrinked to $\mathfrak{K}$ is dense in $\mathrm{C}^{0}(\mathfrak{K})$ : Since $\mathfrak{K}$ is compact and Hausdorff, the Stone-Weierstraß theorem [Dud02, Theorem 2.4.11] implies that any subalgebra of $\mathrm{C}^{0}(\mathfrak{K})$ that contains the constants and separates points is dense in $\mathrm{C}^{0}(\mathfrak{K})$. Such a subalgebra is given by $\eta_{\mathfrak{M}}\left(\mathrm{C}^{0,1}(\mathfrak{M})\right)$ as all constants are of the form $\int$ const $\mathrm{d}($.$) and, according to Dud02, Theorem 11.3.3], the Hausdorff topology$ of $\mathcal{P} \mathfrak{M}$ is induced by the functions $\left\{\int h \mathrm{~d}(.)\right\}_{h \in \mathrm{C}^{0,1}(\mathfrak{M})}$.

In our case, this observation in combination with the assumption that $\overline{\mathbb{M}_{\varphi}}$ is compact amounts to the fact that the algebra generated by the functions $\left\{\int h \mathrm{~d}(.)\right\}_{h}$, where $h \in \mathrm{C}^{0,1}(\mathrm{PM})$, is dense in $\mathrm{C}^{0}\left(\overline{\mathbb{M}_{\varphi}}\right)$ with respect to the sup norm. Hence $\phi$ can be approximated by a finite superposition of finite products

$$
\phi \approx P \mapsto \sum_{i} c_{i} \prod_{j} \int h_{i j} \mathrm{~d} P
$$

with error $<\varepsilon / 2$ for some Lipschitz continuous functions $h_{i j}$ on PM uniformly bounded by 1 .

Let $c=\sum\left|c_{i}\right|$ and $m$ be the number of all multi-indices $(i, j)$. If we manage to estimate $\int h_{i j} \mathrm{~d} P_{M}$ with error less than $c^{-1} \varepsilon / 2$ with probability more than $1-$ $\varepsilon / m$, the proof is completed: More formally, we seek a tester $\tau_{i j}=\tau_{i j}^{n}:(\mathrm{PM})^{n} \rightarrow$ $\mathbb{R}$ bounded uniformly by 1 such that

$$
\operatorname{Prob}\left(\left|\int h_{i j} \mathrm{~d} P_{M}-\tau_{i j}\left(B_{r}^{n}\right)\right|>\frac{1}{2 c} \varepsilon\right)<\frac{1}{m} \varepsilon .
$$

If so, for $\tau=\sum_{i} c_{i} \prod_{j} \tau_{i j}$ we gain the bound

$$
\begin{aligned}
\operatorname{Prob} & \left(\left|\phi(M)-\tau\left(B_{r}^{n}\right)\right|>\varepsilon\right) \\
& \leq \operatorname{Prob}\left(\left|\sum_{i} c_{i} \prod_{j} \int h_{i j} \mathrm{~d} P_{M}-\tau\left(B_{r}^{n}\right)\right|>\varepsilon / 2\right) \\
& \leq \operatorname{Prob}\left(\left|\sum_{i} c_{i}\left(\prod_{j} \int h_{i j} \mathrm{~d} P_{M}-\prod_{j} \tau_{i j}\left(B_{r}^{n}\right)\right)\right|>\varepsilon / 2\right) \\
& =\operatorname{Prob}\left(\left|\sum_{i} c_{i} \sum_{j}\left(\int h_{i j} \mathrm{~d} P_{M}-\tau_{i j}\left(B_{r}^{n}\right)\right) \prod_{k>j} \int h_{i k} \mathrm{~d} P_{M} \prod_{k<j} \tau_{i k}\left(B_{r}^{n}\right)\right|>\varepsilon / 2\right) \\
& \leq \operatorname{Prob}\left(\sum_{i} c_{i} \sum_{j}\left|\int h_{i j} \mathrm{~d} P_{M}-\tau_{i j}\left(B_{r}^{n}\right)\right| \prod_{k>j}\left\|h_{i k}\right\|_{\mathrm{C}^{0}} \mid \prod_{k<j}\left\|\tau_{i k}\right\|_{\mathrm{C}^{0}}>\varepsilon / 2\right) \\
& \leq \operatorname{Prob}\left(\sum_{i} c_{i} \sum_{j}\left|\int h_{i j} \mathrm{~d} P_{M}-\tau_{i j}\left(B_{r}^{n}\right)\right|>\varepsilon / 2\right) \\
& \leq \operatorname{Prob}\left(\left|\int h_{i j} \mathrm{~d} P_{M}-\tau_{i j}\left(B_{r}^{n}\right)\right|>c^{-1} \varepsilon / 2 \quad \text { for some multi-index }(i, j)\right) \\
& \leq m \cdot \varepsilon / m=\varepsilon .
\end{aligned}
$$

We now seek a tester $\tau_{i j}$ satisfying (2.1). First compare the functions $h_{i j}$ and $h_{i j} \circ s_{r}: \mathrm{PM} \rightarrow \mathbb{R}$ given by $h_{i j} \circ s_{r}(M$, vol,$p)=h_{i j}\left(\mathrm{~B}[p, r],\left.\operatorname{vol}\right|_{\mathrm{B}[p, r]}, p\right)$. From the Definition 28 in Bow15. we read that for any $\varepsilon^{\prime}$, the pointed spaces $M$ and $s_{r}(M)$ are $\varepsilon^{\prime}$-close for sufficiently large $r$ with respect to the metric constructed in the proof of Theorem 3.1 therein. This $r$ is independent of the choice of $M \in \mathrm{PM}$. Observe further that being Lipschitzian $h_{i j}$ is uniformly continuous. Choose $r$ such that for all $i, j$ the distance $\left\|h_{i j}-\tau_{i j} \circ s_{r}\right\|_{\mathrm{C}^{0}}$ is smaller than $c^{-1} \varepsilon / 4$. Now take $M_{1}, \ldots, M_{n}$ in the image of $s_{r}$ and set the real-valued map

$$
\tau_{i j}^{n}\left(\left(M_{k}\right)_{k=1}^{n}\right):=\frac{1}{n} \sum_{k=1}^{n} h_{i j}\left(M_{k}\right)=\frac{1}{n} \sum_{k=1}^{n} h_{i j}\left(s_{r} M_{i}\right) .
$$


To finish the proof, the $\tau_{i j}^{n}$ have to converge uniformly with respect to the tested manifold $M$. Here basic probability theory enters the proof:

$$
\begin{aligned}
& \operatorname{Prob}\left(\left|\int h_{i j} \mathrm{~d} P_{M}-\tau_{i j}\left(B_{r}^{n}\right)\right|>c^{-1} \varepsilon / 2\right) \\
& \leq \operatorname{Prob}(\underbrace{\left|\int h_{i j} \mathrm{~d} P_{M}-\int \tau_{i j}\left(B_{r}^{n}\right) \mathrm{d} P_{M}\right|}_{\leq c^{-1} \varepsilon / 4}+|\underbrace{\int \tau_{i j}\left(B_{r}^{n}\right) \mathrm{d} P_{M}}_{=\mathrm{E} \tau_{i j}\left(B_{r}^{n}\right)}-\tau_{i j}\left(B_{r}^{n}\right)|>\frac{\varepsilon}{2 c}) \\
& \quad \leq \operatorname{Prob}\left(\left|\left(\mathrm{E} \tau_{i j}\left(B_{r}^{n}\right)\right)-\tau_{i j}\left(B_{r}^{n}\right)\right|>c^{-1} \varepsilon / 4\right)
\end{aligned}
$$

Chebyshev inequality FG97, Chapter 5 Proposition 2] implies

$$
\begin{array}{llrl}
\leq 16 c^{2} \varepsilon^{-2} \operatorname{Var} \tau_{i j}\left(B_{r}^{n}\right) & & \text { by definition from } 2.2 \\
=16 c^{2} \varepsilon^{-2} \operatorname{Var} n^{-1} \sum_{k=1}^{n} h_{i j} \circ \operatorname{pr}_{k}\left(B_{r}^{n}\right) & & \text { pairwise uncorrelated } \\
& =16 c^{2} \varepsilon^{-2} n^{-2} \sum_{k=1}^{n} \operatorname{Var} h_{i j} \circ \operatorname{pr}_{k}\left(B_{r}^{n}\right) & & \text { since }\left\|h_{i j}\right\|_{\mathrm{C}^{0}} \leq 1 \\
\leq 16 c^{2} \varepsilon^{-2} n^{-2} \cdot n \cdot 4 & & \\
=64 c^{2} \varepsilon^{-2} n^{-1} . & &
\end{array}
$$

Therefore by sufficiently large choice of $n$ condition 2.1 holds.

\subsection{Characteristic numbers}

In this section we will formulate a condition under which characteristic numbers are testable. We introduce characteristic numbers by Chern-Weyl theory MS74 Appendix $C]$ : Let $\Pi$ be an invariant polynomial on the matrix algebra $\mathrm{M}_{d}(\mathbb{C})$, i.e. a map $\mathrm{M}_{d}(\mathbb{C}) \rightarrow \mathbb{C}$ that is described by a polynomial in the entries of the matrix and is invariant under base change. For any connection $\nabla$ on a manifold $M$ let $R_{\nabla}$ be the corresponding curvature tensor. The characteristic number of an even-dimensional compact Riemannian $d$-manifold $M$ (without boundary) with respect to $\Pi$ (and $\nabla$ ) is given by an integral

$$
\int_{M} \Pi\left(R_{\nabla}\right)[M]
$$

where $[M]$ is the fundamental class and $R_{\nabla}$ is considered as a $d \cdot d$-matrix valued in 2-forms and the resulting map to $\mathbb{C}$ is well-defined due to invariance of $\Pi$. Note, that as we evaluate with respect to the fundamental class characteristic numbers actually change sign with change of orientation. These numbers turn out to be independent of the connection. Let $\Gamma_{i j}$ be the Christoffle symbols of the connection. Recall that in coordinates $\left(R_{\nabla}\right)_{i j k}^{l}=\partial_{j} \Gamma_{i k}^{l}-\partial_{k} \Gamma_{i j}^{l}+\sum_{s} \Gamma_{j s}^{l} \Gamma_{i k}^{s}-$ $\Gamma_{k s}^{l} \Gamma_{i j}^{s}$ Moreover the polynomial function $\Pi$ is of degree $d / 2$ in the components of $R_{\nabla}$.

Characteristic numbers are a vast generalization of the Euler characteristic of a surface, which by the Gauss-Bonnet Theorem can expressed by an integral

$$
\chi(M)=\frac{1}{2 \pi} \int K \mathrm{~d} v o l
$$

where $K$ denotes the Gaussian curvature. In our definition any characteristic number of a non-orientable manifold vanishes. There are many special kinds of characteristic numbers studied, like Chern numbers and Pontryagin numbers. As in case of the Euler characteristic these numbers often give integer values for 
any Riemannian manifold $M$. One can even give explicitly a base for the vector space of invariant polynomials such that each base vector corresponds to an integer valued characteristic number. Characteristic numbers are an important invariant in the study of oriented cobordism.

\subsubsection{Characteristic numbers from rough connections}

We want to define the curvature tensor of a connection in a setup of a manifold $M$ with a locally finite atlas $\varphi_{i}: \mathrm{B}(0, \varrho)$ of regularity $\mathrm{C}^{2, \alpha}$. Such an atlas can carry at most a $\mathrm{C}^{1, \alpha}$ regular tensor, but only a $\mathrm{C}^{\alpha}$-regular connection because the transition function $\varphi_{i}^{-1} \circ \varphi$ from a chart $\psi:\left(V,{ }^{\psi} \Gamma_{\mu \nu}^{k}\right) \rightarrow M$ to some chart $\varphi: U \rightarrow M$ involves second derivatives. It is given by

$$
\begin{array}{r}
\left.{ }^{\varphi} \Gamma_{\mu \nu}^{k}=\left(\varphi^{-1} \circ \psi\right)_{*}\left({ }^{\psi} \Gamma_{. .}^{.}\right)\right) \\
=\sum_{\mu^{\prime}, \nu^{\prime}, k^{\prime}} \frac{\partial y_{\mu^{\prime}}}{\partial x_{\mu}} \frac{\partial y_{\nu^{\prime}}}{\partial x_{\nu}}\left({ }^{\psi} \Gamma_{\mu^{\prime} \nu^{\prime}}^{k^{\prime}} \circ y\right)\left(\frac{\partial x_{k}}{\partial x_{k^{\prime}}} \circ y\right) \\
+\sum_{l=1}^{d}\left(\frac{\partial x_{k}}{\partial x_{l}} \circ y\right) \frac{\partial^{2} \psi_{l}^{-1} \circ \varphi}{\partial x_{\mu} \partial x_{\nu}}
\end{array}
$$

where $x=\varphi^{-1} \circ \psi: V \rightarrow U \subset \mathbb{R}^{d}$ and $y=\psi^{-1} \circ \varphi: U \rightarrow V \subset \mathbb{R}^{d}$

$$
=\sum_{\mu^{\prime}, \nu^{\prime}, l} y_{\mu^{\prime}, \mu} y_{\nu^{\prime}, \nu}\left({ }^{\psi} \Gamma_{\mu^{\prime} \nu^{\prime}}^{l} \circ y\right)\left(x_{k, l} \circ y\right)+\sum_{l}\left(x_{k, l} \circ y\right)\left(y_{l, \mu \nu}\right) .
$$

We will however demonstrate how two define a $\mathrm{C}^{\alpha}$-curvature tensor of a connection on such an atlas. To this end set any regularity issues aside for a moment. Take a partition of unity $\left\{\lambda_{i}: M \rightarrow \mathbb{R}\right\}_{i \in I}$ compatible with the atlas, i.e. $\operatorname{supp} \lambda_{i} \subset \varphi_{i}(\mathrm{~B}(0, \varrho))$ and $\sum_{i \in I} \varphi_{i} \equiv 1$. With such a datum $\left(\left\{\varphi_{i}\right\},\left\{\lambda_{i}\right\}\right)$ we define a connection by a convex combination of the Euclidean connections on each chart

$$
\nabla_{\left\{\varphi_{i}\right\},\left\{\lambda_{i}\right\}}=\sum_{i \in I} \lambda_{i}\left(\varphi_{i}\right)_{*} \nabla_{\text {Eucl. }} .
$$

This makes sense as the convex combination of connections is again a connection. Formula 2.4 simplifies in case $\psi=\varphi_{i}$ and ${ }^{\psi} \Gamma_{\mu \nu}^{k}={ }^{\text {Eucl. }} \Gamma_{\mu \nu}^{k}=0$ to $\sum_{l}\left(x_{k, l} \circ y\right)$ $\left(y_{l, \mu \nu}\right)$. In this notation the Christoffel symbols ${ }^{\varphi} \Gamma_{\mu \nu}^{k}$ of $\nabla_{\left\{\varphi_{i}\right\},\left\{\lambda_{i}\right\}}$ on $\varphi$ are given by

$$
\begin{aligned}
{ }^{\varphi} \Gamma_{\mu \nu}^{k} & =\sum_{i \in I} \lambda_{i}\left(\left(\varphi^{-1} \circ \varphi_{i}\right)_{*}{ }^{\text {Eucl. }} \Gamma_{. .}^{\cdot}\right)_{\mu \nu}^{k}=\sum_{i \in I} \lambda_{i}{ }^{i} \Gamma_{\mu \nu}^{k} \\
& =\sum_{i \in I} \lambda_{i} \sum_{l}\left(x_{k, l}^{i} \circ y^{i}\right)\left(y_{l, \mu \nu}^{i}\right)
\end{aligned}
$$

where ${ }^{i} \Gamma_{\mu \nu}^{k}=\left(\left(\varphi^{-1} \circ \varphi_{i}\right)_{*}{ }^{\text {Eucl. }} \Gamma_{. .} .\right)_{\mu \nu}^{k}, x^{i}=\varphi^{-1} \circ \varphi_{i}$, and $y^{i}=\varphi_{i}^{-1} \circ \varphi$. We formally calculate the curvature tensor on $\varphi$ from the standard coordinate definition

$$
{ }^{\varphi} R_{\lambda \mu \nu}^{k}=\left({ }^{\varphi} \Gamma_{\nu \lambda, \mu}^{k}\right)_{[\mu \nu]}+\sum_{\kappa}\left({ }^{\varphi} \Gamma_{\mu \kappa}^{k}{ }^{\varphi} \Gamma_{\nu \lambda}^{\kappa}\right)_{[\mu \nu]}
$$


where we used the standard shorthand $h_{[i j]}:=h_{i j}-h_{j i}$

$$
\begin{aligned}
= & \sum_{i \in I}\left(\left(\lambda_{i}{ }^{i} \Gamma_{\nu \lambda}^{k}\right)_{, \mu}\right)_{[\mu \nu]}+\sum_{\kappa}\left({ }^{\varphi} \Gamma_{\mu \kappa}^{k}{ }^{\varphi} \Gamma_{\nu \lambda}^{\kappa}\right)_{[\mu \nu]} \\
= & \sum_{i \in I}\left(\lambda_{i}^{i} \Gamma_{\nu \lambda, \mu}^{k}+\lambda_{i, \mu}{ }^{i} \Gamma_{\nu \lambda}^{k}\right)_{[\mu \nu]}+\sum_{\kappa}\left({ }^{\varphi} \Gamma_{\mu \kappa}^{k}{ }^{\varphi} \Gamma_{\nu \lambda}^{\kappa}\right)_{[\mu \nu]} \\
= & \sum_{i \in I} \lambda_{i}\left(\sum_{l}\left(\left(x_{k, l}^{i} \circ y^{i}\right) y_{l, \nu \lambda}^{i}\right)_{, \mu}\right)_{[\mu \nu]} \\
& \quad+\left(\lambda_{i, \mu}{ }^{i} \Gamma_{\nu \lambda}^{k}\right)_{[\mu \nu]}+\sum_{\kappa}\left({ }^{\varphi} \Gamma_{\mu \kappa}^{k} \Gamma_{\nu \lambda}^{\kappa}\right)_{[\mu \nu]} \\
= & \sum_{i \in I} \lambda_{i} \sum_{l}\left(\left(x_{k, l}^{i} \circ y^{i}\right)_{, \mu} y_{l, \nu \lambda}^{i}\right)_{[\mu \nu]}+\underbrace{\left(\left(x_{k, l}^{i} \circ y^{i}\right)_{l, \nu \lambda \mu}^{i}\right)_{[\mu \nu]}}_{=0} \\
& +\left(\lambda_{i, \mu}{ }^{i} \Gamma_{\nu \lambda}^{k}\right)_{[\mu \nu]}+\sum_{\kappa}\left({ }^{\varphi} \Gamma_{\mu \kappa}^{k} \Gamma_{\nu \lambda}^{\kappa}\right)_{[\mu \nu]} \\
= & \sum_{i \in I} \lambda_{i} \sum_{l}\left(\left(x_{k, l}^{i} \circ y^{i}\right)_{, \mu} y_{l, \nu \lambda}^{i}\right)_{[\mu \nu]}+\left(\lambda_{i, \mu}^{i} \Gamma_{\nu \lambda}^{k}\right)_{[\mu \nu]} \\
& +\sum_{\kappa}\left({ }^{\varphi} \Gamma_{\mu \kappa}^{k}{ }^{\varphi} \Gamma_{\nu \lambda}^{\kappa}\right)_{[\mu \nu]} .
\end{aligned}
$$

The trick is now to define $\nabla_{\left\{\varphi_{i}\right\},\left\{\lambda_{i}\right\}}$ not by 2.5 , which does not exist, but by formula 2.8. The only remaining thing to check is that this defines a tensor, i.e. is coordinate independent. The following lemma expresses this fact.

Lemma 2.12. Given a cover of a manifold $M$ by charts $\varphi_{i}: U_{i} \rightarrow M$ in the $\mathrm{C}^{2}$-atlas of $M$ with a corresponding $\mathrm{C}^{1}$-partition of unity $\lambda_{i}$. For any two charts $\varphi: U \rightarrow M$ and $\varphi^{\prime}: U^{\prime} \rightarrow M$ the expression defined by (2.8) coincide on $\varphi(U) \cap \varphi^{\prime}\left(U^{\prime}\right)$ as (3,1)-tensor, i.e.

$$
\sum_{\lambda^{\prime}, \mu^{\prime}, \nu^{\prime}, k^{\prime}}\left({ }^{\prime} R_{\lambda^{\prime} \mu^{\prime} \nu^{\prime}}^{k^{\prime}} \circ x^{\prime}\right) x_{\lambda^{\prime}, \lambda}^{\prime} x_{\mu^{\prime}, \mu}^{\prime} x_{\nu^{\prime}, \nu}^{\prime}\left(x_{k, k^{\prime}} \circ x^{\prime}\right)={ }^{\varphi} R_{\lambda \mu \nu}^{k} .
$$

The proof is given in appendix $\mathrm{B}$ and consists in an algebraic calculation, that is more involved than the standard textbook calculations because it has to avoid any third derivative of transition functions, not to mention derivatives of the metric tensor or Christoffel symbols.

Lemma 2.13. The coordinate function $(2.8)$ is $\mathrm{C}^{\alpha}$-bounded provided that the transition functions involved are $\mathrm{C}^{2, \alpha}$-bounded and that the partition of unity is $\mathrm{C}^{1, \alpha}$-bounded.

Proof. This is a direct consequence of the product estimate Corollary 1.11 and the concatenation estimate Lemma 1.13

Definition 2.14. Let $\left\{\varphi_{i}\right\}$ be a locally finite atlas of $M$ and $\left\{\lambda_{i}\right\}$ a corresponding partition of unity. We say that the function on a chart $\varphi$ defined by $(2.8)$ is the curvature tensor of the piecewise Euclidean connection $\nabla_{\left\{\varphi_{i}\right\},\left\{\lambda_{i}\right\}}$ as defined in 2.5 and denote it by $R_{\nabla_{\left\{\varphi_{i}\right\},\left\{\lambda_{i}\right\}}}=R\left(\left\{\varphi_{i}\right\},\left\{\lambda_{i}\right\}\right)$. 
Lemma 2.15. For a closed oriented manifold $M$ and any invariant polynomial $\Pi$ the characteristic number

$$
\int_{M} \Pi\left(R\left(\left\{\varphi_{i}\right\},\left\{\lambda_{i}\right\}\right)\right)[M]:=\sum_{i} \int_{U_{i}} \mu_{i} \circ \psi_{i} \cdot \Pi\left({ }^{\psi_{i}} R_{\lambda \mu \nu}^{k}\right) \mathrm{d} x,
$$

where $\psi_{i}: U_{i} \rightarrow M$ is some $\mathrm{C}^{2}$-atlas compatible with the orientation and $\mu_{i}$ a corresponding smooth partition of unity, is well-defined, independent of the choice of a curvature tensor given by a piecewise Euclidean connection, and coincides with the characteristic number given by smooth connection as defined in (2.3).

Proof. It is a standard result that the lemma holds for smooth connections, see MS74, p. 298]. As proven in the mentioned reference, MS74, Appendix C], in the case of smooth connections even the entire de Rham class of the form $\Pi\left(R_{\nabla}\right)$ is independent of the connection. This result extends using mollification:

As a manifold, $M$ is metrizable. Hence we can assume that $M$ carries some metric $d$. Let $\mathrm{d}_{\mathrm{H}}$ denote the Hausdorff distance with respect to $d$. For any chart $\varphi_{i}$ choose some $\varepsilon_{i}<\mathrm{d}_{\mathrm{H}}\left(\operatorname{supp} \lambda_{i}, \partial \varphi_{i}(\mathrm{~B}(0, \varrho))\right)$. For any chart $\varphi_{i}: \underline{\mathrm{B}(0, \varrho) \rightarrow}$ $V_{i} \subset M$ we can define a mollification by choosing a finite cover $V_{i j}$ of $\overline{\varphi(\mathrm{B}(0, \varrho))}$ corresponding to a finite set of smooth charts $\left\{\psi_{i j}: U_{i j} \rightarrow V_{i j} \subset M\right\}_{j \in J_{i}}$ for $\varphi(\mathrm{B}(0, \varrho))$. Moreover we choose corresponding functions $\left\{\mu_{i j}: M \rightarrow[0,1]\right\}_{j \in J_{i}}$ that are a partition on unity for $\operatorname{supp} \lambda_{i}$, i.e.

$$
\begin{aligned}
\operatorname{supp} \mu_{i j} & \subset V_{i j}, & & \text { for all } j \\
\bigcup_{j} \operatorname{supp} \mu_{i j} & \subset \varphi(\mathrm{B}(0, \varrho)), & & \text { and } \\
\operatorname{supp}\left(\lambda_{i}\right)^{\left.\varepsilon_{i}\right)} & \subset\left(\sum_{j} \mu_{i j}\right)^{-1}(\{1\}) & &
\end{aligned}
$$

for all $i$. On each chart $\psi_{i j}$ we mollify the compactly supported function $\mu_{i j} \varphi_{i}^{-1}: U_{i j} \rightarrow \mathbb{R}^{d}$ by convolution on the chart $\psi_{i j}$ with a function $\phi_{\delta}$ which has support in $\mathrm{B}[0, \delta]$ and satisfies $\int \phi_{\delta} \mathrm{d} x=1$, see Hör12, Lemma 1.2.3] for existence. The resulting function

$$
\tilde{\varphi}_{i j}^{\delta}(x):=\int_{\mathbb{R}^{d}} \phi_{\delta}(\xi-x) \cdot \mu_{i j}\left(\psi_{i j}(\xi)\right) \varphi_{i}^{-1}\left(\psi_{i j}(\xi)\right) \mathrm{d} \xi
$$

has support in $V_{i j}$ for $\delta<\delta_{j}:=\mathrm{d}_{\mathrm{H}}\left(\operatorname{supp} \mu_{i j}, \partial U_{i j}\right) \neq 0$. Summing up we define a function $M \rightarrow \mathbb{R}$

$$
\tilde{\varphi}_{i}^{\delta}(x):=\sum_{j} \tilde{\varphi}_{i j}^{\delta}\left(\psi_{i j}^{-1}(x)\right)
$$

which is well defined for $\delta<\min _{j} \delta_{j} \neq 0$. Moreover this function has the property that for sufficiently small $\delta$ the support is contained in $\varphi(\mathrm{B}(0, \varrho))$. Finally, we observe that by standard mollification results Hör12, Theorem 1.3.2] the functions $\tilde{\varphi}_{i j}^{\delta}$ are smooth and $\mathrm{C}^{2}$-converges to $\varphi_{i}^{-1} \circ \psi_{i j}$ as $\delta \rightarrow 0$. Therefore $\tilde{\varphi}_{i}^{\delta}$ converges in $\mathrm{C}^{2}$ to $\varphi_{i} \cdot \sum_{j} \mu_{i j}$ as $\delta \rightarrow 0$.

In a similar fashion we define, for sufficiently small $\delta$, the mollification of the partition of unity $\left\{\lambda_{i}\right\}$

$$
\begin{aligned}
& \tilde{\lambda}_{i j}^{\delta}(x):=\int_{\mathbb{R}^{d}} \phi_{\delta}(\xi-x) \cdot \mu_{i j}\left(\psi_{i j}(\xi)\right) \lambda_{i}\left(\psi_{i j}(\xi)\right) \mathrm{d} \xi \\
& \tilde{\tilde{\lambda}}_{i}^{\delta}(x):=\sum_{j} \tilde{\lambda}_{i j}^{\delta}\left(\psi_{i j}^{-1}(x)\right) .
\end{aligned}
$$


To preserve the partition of unity property we normalize with respect to all indices $i$

$$
\tilde{\lambda}_{i}^{\delta}(x):=\frac{\tilde{\tilde{\lambda}}_{i}^{\delta}(x)}{\sum_{i} \tilde{\tilde{\lambda}}_{i}^{\delta}(x)}
$$

and observe that $\tilde{\lambda}_{i}^{\delta}$ converges in $\mathrm{C}^{2}$ to $\lambda_{i} \cdot \sum_{j} \mu_{i j}=\lambda_{i}$ as $\delta \rightarrow 0$. For each $i$ we can choose $\delta>0$ sufficiently small so that $\operatorname{supp} \tilde{\lambda}_{i}^{\delta} \subset \operatorname{supp}\left(\lambda_{i}\right)^{\left.\varepsilon_{i}\right)}$.

Now we can define the mollified connection: The maps $\tilde{\varphi}_{i}^{\delta}$ is a diffeomorphism from $\left(\sum_{j} \mu_{i j}\right)^{-1}(\{1\})$ onto a subset of $\mathbb{R}^{d}$ and hence diffeomorphism on supp $\tilde{\lambda}_{i}^{\delta}$. Thus we can define a smooth connection on $M$ given by

$$
\nabla^{\delta}:=\sum_{i j} \tilde{\lambda}_{i}^{\delta}\left(\tilde{\varphi}_{i}^{\delta}\right)^{*} \nabla_{\text {Eucl }}
$$

that is well-defined for small $\delta$ because for any point $x$ there are only finitely $i$ such that $x \in \varphi_{i}(\mathrm{~B}(0, \varrho))$.

Now we can check the claim on characteristic numbers. For a compact manifold $M$ the locally finite collection of charts $\left\{\varphi_{i}\right\}$ is actually finite (given a locally finite cover $V_{i}, i \in I$, one can find for each point an open neighborhood $U_{x}$ that is contained in only finitely $V_{i}$ 's. By compactness the space is covered by only finitely many $U(x)$ 's, let's say $U\left(x_{1}\right), \ldots, U\left(x_{n}\right)$. Thereby one can bound $I$ by $n$ times the maximal number of $V_{i}$ 's to which one of the $x_{1}, \ldots, x_{n}$ belongs). Hence we can choose $\delta>0$ such that for all $i$ the mollified functions $\tilde{\varphi}_{i}^{\delta}$ and $\tilde{\lambda}_{i}^{\delta}$ are defined. Therefore

$$
\begin{aligned}
\int_{M} \Pi\left(R_{\nabla_{\left\{\varphi_{i}\right\},\left\{\lambda_{i}\right\}}}\right) & =\sum_{i} \int_{U_{i}} \mu_{i} \circ \psi_{i} \cdot \Pi\left({ }^{\psi_{i}} R_{\lambda \mu \nu}^{k}\right) \mathrm{d} x \\
& =\lim _{\delta \rightarrow 0} \sum_{i} \int_{U_{i}} \mu_{i} \circ \psi_{i} \cdot \Pi\left({\frac{\nabla_{i}}{\delta}}_{R_{\lambda \mu \nu}}^{k}\right) \mathrm{d} x \\
& =\int_{M} \Pi\left(R_{\nabla^{\delta}}\right) .
\end{aligned}
$$

This proves that $\int_{M} \Pi\left(R_{\nabla_{\left\{\varphi_{i}\right\},\left\{\lambda_{i}\right\}}}\right)$ equals the actually characteristic number of $M$ with respect to $\Pi$ and is therefore independent of the choice of the atlas $\left\{U_{i}\right\}$.

\subsubsection{Testability from random connections}

Extending Definition 2.2 we define unimodularity of curvature tensors by the Intrinsic Mass-Transport Principle:

Definition 2.16. Let $0 \leq \alpha^{\prime} \leq \alpha$. Let further be

$$
P \in \mathcal{P}\left(\mathrm{T}_{\mathrm{C}^{\alpha^{\prime}} \leq \Theta^{\prime}}^{3,1} \mathrm{P}_{\mathcal{M}^{m, \alpha} \leq \Theta}^{d, \varrho}\right) .
$$

The law $P$ is said to be a unimodular tensor if the measures $m_{\mathrm{L}}(P)$ and $m_{\mathrm{R}}(P)$ are equal where the maps

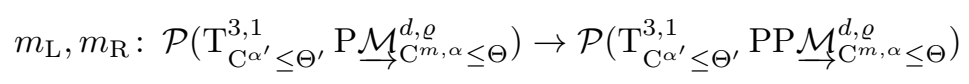


are induced by Lemma 1.7 and the maps

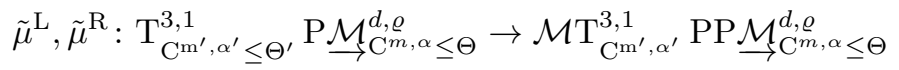

defined by

$$
\begin{array}{llll}
\tilde{\mu}^{\mathrm{L}}:(M, p, \operatorname{vol}, R) & \mapsto\left(\mu_{M}^{\mathrm{L}}\right)_{*} \text { vol } & \text { where } & \mu^{\mathrm{L}}: x \mapsto(M, p, x) \\
\tilde{\mu}^{\mathrm{R}}:(M, p, \operatorname{vol}, R) \mapsto\left(\mu_{M}^{\mathrm{R}}\right)_{*} \text { vol } & \text { where } & \mu^{\mathrm{R}}: x \mapsto(M, x, p) . & \text { and }
\end{array}
$$

Moreover $P$ is called a random curvature tensor if the random (3,1)tensor $R$ of $(M, p, R) \in \mathrm{T}_{\mathrm{C}^{\alpha^{\prime}} \leq \Theta^{\prime}}^{3,1} \stackrel{\mathrm{P}}{\mathcal{M}_{\mathrm{C}}^{m, \alpha} \leq \Theta}{ }^{d, \varrho}$ is $P$-a.s. ( $P$ almost surely) induced by a connection, i.e. it is $P$-a.s. the case that either there is a connection $\nabla$ of class at least $\mathrm{C}^{1}$ on $M$ inducing $R$ or there is a piecewise Euclidean connection $\nabla_{\left\{\varphi_{i}\right\},\left\{\lambda_{i}\right\}}$ inducing $R$ by formula 2.8 .

Finally, $P$ is unimodular curvature tensor if it is both, unimodular and a random curvature tensor.

As in case of Definition 2.2 the assignments $m_{\mathrm{L}}$ and $m_{\mathrm{R}}$ are continuous.

Lemma 2.17. For any $d \geq 1, \varrho>0, \Theta>0$, and $r \in\left(0, e^{-\Theta} \varrho\right]$ there is a constant $v=v(d, \varrho, \Theta, r)>0$ such that $\operatorname{vol}(\mathrm{B}[x, r]) \geq v$ for any $M$ with $\|M\|_{\mathrm{C}^{\alpha}, \varrho} \leq \Theta, x \in M$, and $\alpha \in(0,1]$.

Proof. Choose a chart with $\|\varphi\|_{\mathrm{C}^{\alpha}, \varrho} \leq \Theta$ and $\varphi(0)=x$. We apply condition (i) of Definition 1.20 several times:

$$
\begin{array}{rlrl}
\operatorname{vol}_{M} \mathrm{~B}[x, r] & \geq \operatorname{vol}_{M} \mathrm{~B}[x, \tilde{r}] & & \text { where } \tilde{r}:=\min \left\{r, e^{-\Theta} \varrho\right\} \\
& \geq \operatorname{vol}_{M} \varphi\left(\mathrm{B}\left[0, e^{-\Theta} \tilde{r}\right]\right) & & \text { by } 1.20] \\
& =\int_{\mathrm{B}\left[0, e^{-\Theta} \tilde{r}\right]} \sqrt{\left|\operatorname{det}\left(g_{. .}\right)\right|} \mathrm{d} x & & \\
& \geq \int_{\mathrm{B}\left[0, e^{-\Theta} \tilde{r}\right]} \sqrt{\left|\lambda_{1}\right|^{d}} \mathrm{~d} x &
\end{array}
$$

where $\lambda_{1}$ is the smallest eigenvalue of $g$.. at $x$

$$
\begin{aligned}
& \geq \int_{\mathrm{B}\left[0, e^{-\Theta} \tilde{r}\right]} \sqrt{\left(e^{-\Theta}\right)^{d}} \mathrm{~d} x \\
& =\operatorname{vol}_{\text {Eucl. }}\left(\mathrm{B}\left[0, e^{-\Theta} \tilde{r}\right]\right) \cdot e^{-d \Theta / 2} \quad=: v(d, \varrho, \Theta, r) .
\end{aligned}
$$

Theorem 2.18 (testability). Let $\alpha, \Theta>0$ and $\Pi$ be an invariant polynomial. The normalized characteristic number corresponding to $\Pi$ is the parameter

$$
\varphi_{\Pi}: M \mapsto \frac{1}{\operatorname{vol} M} \int_{M} \Pi\left(R_{\nabla}\right)
$$

where $M$ is a smooth compact Riemannian manifold and $\nabla$ is a connection thereon. The parameter $\varphi_{\Pi}$ is testable in constant time on a relatively compact class $\mathfrak{M} \subset \mathrm{P} \underset{\mathcal{M}^{\alpha}}{d, \varrho} \leq \Theta$ of smooth compact Riemannian d-manifolds $M=\tilde{\mu}(M)$ if

(i) for some $\beta \in(0,1]$ and $\Theta^{\prime}>0$ there is a continuous map $D_{(.)}$

$$
\mathcal{P} P \underline{\mathcal{M}}_{\mathrm{C}^{\alpha} \leq \Theta}^{d, \varrho} \supset \overline{\mathfrak{M}} \stackrel{D}{\rightarrow} \mathcal{P}\left(\mathrm{T}_{\mathrm{C}^{\beta} \leq \Theta^{\prime}}^{3,1}{\stackrel{\mathrm{P}}{\mathcal{M}_{\mathrm{C}}^{\alpha} \leq \Theta}}_{\mathrm{C}^{\alpha}, \varrho}\right)
$$


(ii) for any $P \in \overline{\mathfrak{M}}$ the law $D_{P}$ is a unimodular curvature tensor as defined in Definition 2.16.

(iii) $D$ is a section, i.e.

$$
\mathcal{P}((M, p, R) \mapsto(M, p)) \circ D=\mathrm{id} ;
$$

where $(M, p, R) \mapsto(M, p)$ is a map from $\mathrm{T}_{\mathrm{C}^{\beta} \leq \Theta^{\prime}}^{3,1} \mathrm{P}_{\mathcal{M}^{\alpha} \leq \Theta}^{d, \varrho}$ to the space $\mathrm{P} \underset{\mathcal{M}_{\mathrm{C}}^{d,}}{d, \varrho} \leq \Theta$.

Proof. Define

$$
\chi_{x}^{v}(y)= \begin{cases}1 & \text { if } \operatorname{vol}_{M} \mathrm{~B}(y, d(x, y)) \leq v \\ 0 & \text { else. }\end{cases}
$$

We write $\chi_{x}^{v}(y ; g)$ if we want to make the metric on which $d$ depends explicit. By Lemma 2.17 choose $v$ such that $\chi_{p}^{v}$ is supported on $\mathrm{B}\left[p, e^{-\Theta} \varrho\right]$.

Let $M=(M, g, v o l) \in \mathfrak{M}$ be any Riemannian $d$-manifold and let $R$ be the curvature of a connection $\nabla$ of class $\mathrm{C}^{\alpha}$, e.g. let $g$ be smooth and $\nabla$ be the LeviCivita connection. Such an $R$ always exists since one can always find a smooth metric on a differentiable manifold. Note that the quotient $\frac{\Pi(R)}{\omega}: M \rightarrow \mathbb{R}$ of the evaluation of $\Pi$ at $R$ and the volume form $\omega$ is well defined since $M$ is oriented. Note that

$$
\frac{1}{v} \iint \chi_{p}^{v}(x) \mathrm{d} v o l^{\prime}(p) \mathrm{d} D_{M}\left(M^{\prime}, v o l^{\prime}, p^{\prime}, R^{\prime}\right)=\frac{1}{v} \int v \mathrm{~d} D_{M}\left(M^{\prime}, v o l^{\prime}, p^{\prime}, R^{\prime}\right)=1
$$

Using this we calculate the normalized characteristic number:

$$
\begin{aligned}
& \frac{1}{\operatorname{vol} M} \int_{M} \frac{\Pi(R(x))}{\omega} \mathrm{d} \operatorname{vol}(x) \\
& =\frac{1}{\operatorname{vol} M} \int \frac{\Pi(R(x))}{\omega}\left(\frac{1}{v} \iint \begin{array}{c}
\chi_{p}^{v}(x) \mathrm{d} v o l^{\prime}(p) \\
\mathrm{d} D_{M}\left(M^{\prime}, v o l^{\prime}, p^{\prime}, R^{\prime}\right)
\end{array}\right) \mathrm{d} v o l(x) \\
& =\quad \frac{1}{\operatorname{vol} M} \int \frac{\Pi(R(x))}{\omega} \frac{1}{v} \iint \chi_{p}^{v}(x) \mathrm{d} \operatorname{vol}(p) \mathrm{d} D_{M}\left(M^{\prime}, p^{\prime}, R^{\prime}\right) \mathrm{d} \operatorname{vol}(x) \\
& =\frac{1}{v} \int \frac{1}{\operatorname{vol} M} \iint \frac{\Pi(R(x))}{\omega} \chi_{p}^{v}(x) \mathrm{d} \operatorname{vol}(x) \mathrm{d} \operatorname{vol}(p) \mathrm{d} D_{M}\left(M^{\prime}, p^{\prime}, R^{\prime}\right) \\
& \stackrel{\text { Lemma }}{=} \underset{2.15}{\frac{1}{v}} \int \frac{1}{\operatorname{vol} M} \iint \frac{\Pi\left(R^{\prime}(x)\right)}{\omega} \chi_{p}^{v}(x) \mathrm{d} \operatorname{vol}(x) \mathrm{d} \operatorname{vol}(p) \mathrm{d} D_{M}\left(M^{\prime}, p^{\prime}, R^{\prime}\right) \\
& \stackrel{\text { Lemma } 1.7}{=} \frac{1}{v} \int \frac{1}{\operatorname{vol} M} \int \frac{\Pi\left(R^{\prime}(x)\right)}{\omega} \chi_{p}^{v}(x) \mathrm{d} \operatorname{vol}(x) \mathrm{d} m_{\mathrm{R}}\left(D_{M}\right)\left(M^{\prime}, p, p^{\prime}, R^{\prime}\right) \\
& \stackrel{\text { unimodularity }}{=} \frac{1}{v} \int \frac{1}{\operatorname{vol} M} \int \frac{\Pi\left(R^{\prime}(x)\right)}{\omega} \chi_{p}^{v}(x) \mathrm{d} \operatorname{vol}(x) \mathrm{d} m_{\mathrm{L}}\left(D_{M}\right)\left(M^{\prime}, p, p^{\prime}, R^{\prime}\right)
\end{aligned}
$$

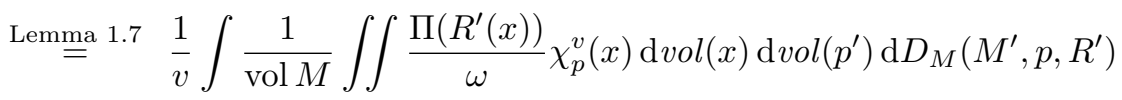

$$
\begin{aligned}
& =\quad \frac{1}{v} \iint \frac{\Pi\left(R^{\prime}(x)\right)}{\omega} \chi_{p}^{v}(x) \mathrm{d} \operatorname{vol}(x) \mathrm{d} D_{M}\left(M^{\prime}, p, R^{\prime}\right)
\end{aligned}
$$

In the final expression the inner integrand does only depend on a bounded ball around the base point. Hence the integrand $\int \frac{\Pi\left(R^{\prime}(x)\right)}{\omega} \chi_{p}^{v}(x) \mathrm{d} \operatorname{vol}(x)$ is a continuous as function of $\left(M^{\prime}, p^{\prime}, R^{\prime}\right)$ on the entire closure of $\mathfrak{M}$. 
It remains to check that the integrand in bounded. By assumption (i) we can almost surely choose a chart $\varphi$ around $p$ such that $\|\varphi\|_{\mathrm{C}^{\alpha}} \leq \Theta$, the tensor $R^{\prime}$ in bounded in $\mathrm{C}^{0}$-norm on this chart. Thus $\chi_{p}^{v}$ is supported on the image of this chart. Hence for $\left(M^{\prime}, p, R^{\prime}\right)=\left(M^{\prime}, g^{\prime}, p, R^{\prime}\right)$ we have a bound

$$
\begin{aligned}
\left|\int \frac{\Pi\left(R^{\prime}(x)\right)}{\omega} \chi_{p}^{v}(x) \mathrm{d} \operatorname{vol}(x)\right| & =\left|\int_{\mathrm{B}(0, \varrho)} \Pi\left(\varphi^{*} R^{\prime}\right)(x) \chi_{p}^{v}\left(x ; \varphi^{*} g^{\prime}\right) \sqrt{|\operatorname{det} g . .|} \mathrm{d} x\right| \\
& \leq\left\|\Pi\left(\varphi^{*} R^{\prime}\right)\right\|_{\mathrm{C}^{0}} \cdot \int_{\mathrm{B}(0, \varrho)} \sqrt{|\operatorname{det} g . .|} \mathrm{d} x \\
& \leq\left\|\Pi\left(\varphi^{*} R^{\prime}\right)\right\|_{\mathrm{C}^{0}} \cdot \int_{\mathrm{B}(0, \varrho)} \sqrt{\lambda_{d}^{d}} \mathrm{~d} x
\end{aligned}
$$

where $\lambda_{d}$ is the largest eigenvalue of $g$.. at $x$

$$
\begin{aligned}
& \leq\left\|\Pi\left(\varphi^{*} R^{\prime}\right)\right\|_{\mathrm{C}^{0}} \cdot \int_{\mathrm{B}(0, \varrho)} \sqrt{\left(e^{\Theta}\right)^{d}} \mathrm{~d} x \\
& =\left\|\Pi\left(\varphi^{*} R^{\prime}\right)\right\|_{\mathrm{C}^{0}} \cdot \operatorname{vol}_{\text {Eucl. }}(\mathrm{B}(0, \varrho)) \cdot e^{d \Theta / 2}
\end{aligned}
$$

in terms of $\varrho, \Theta$, and $\Theta^{\prime}$.

Thus the parameter $\varphi_{\Pi}(M)$ can be expressed by the integral over a bounded and continuous function on $\mathrm{T}_{\mathrm{C}^{\beta} \leq \Theta^{\prime}}^{3,1} \stackrel{\mathrm{P}}{\mathcal{M}_{\mathrm{C}^{\alpha}}^{d, \varrho} \leq \Theta}$. Hence by definition of convergence of laws the assignment

$P \mapsto \frac{1}{v} \iint \frac{\Pi\left(R^{\prime}(x)\right)}{\omega} \chi_{p}^{v}(x) \mathrm{d} \operatorname{vol}(x) \mathrm{d} P\left(M^{\prime}, p, R^{\prime}\right), \quad \mathcal{P} \mathrm{T}_{\mathrm{C}^{\beta} \leq \Theta^{\prime}}^{3,1} \underline{\mathcal{M}}_{\mathrm{C}^{\alpha} \leq \Theta}^{d, \varrho} \rightarrow \mathbb{R}$

is a continuous function. Due to continuity of $D$ the concatenation

$$
P \mapsto \frac{1}{v} \iint \frac{\Pi\left(R^{\prime}(x)\right)}{\omega} \chi_{p}^{v}(x) \mathrm{d} \operatorname{vol}(x) \mathrm{d} D_{P}\left(M^{\prime}, p, R^{\prime}\right), \quad \overline{\mathfrak{M}} \rightarrow \mathbb{R}
$$

is continuous as well and is the extension of the parameter $\varphi_{\Pi}$ we sought.

\subsubsection{A first analytic criterion}

We can immediately give two criteria using which we can apply Theorem 2.18 The first is analytic.

Theorem 2.19. Let $\varrho, \Theta \in(0, \infty)$ and $0<\beta<\alpha \leq 1$. The parameter $\varphi_{\Pi}$, as defined in Theorem 2.18, is testable in constant time on the class $\mathfrak{M}$ of compact spaces in $\mathcal{P} \mathrm{P} \underset{\mathcal{M}^{2, \alpha} \leq \Theta}{d, \varrho}$.

In view of this theorem, the main theorem, Theorem 2.22 we are going to prove in the next section can be summarized as saving two derivatives compared to the preliminary result above.

Proof. Due to Lemma 1.29 the set

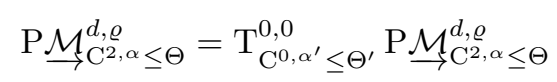

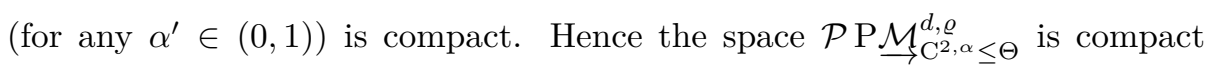
Dud02, Theorem 11.5.4]. 
Now check the assumptions of Theorem 2.18 for $\varphi_{\Pi}$ restricted to $\mathfrak{M}$ : As the

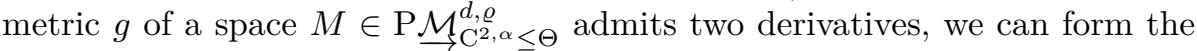
Levi-Civita connection and its Riemannian curvature tensor $R_{\text {Riem. }}$ given by 2.7. . The Christoffel symbols $\Gamma_{i j}^{k}=\sum_{\mu} \frac{1}{2} g^{k \mu}\left(g_{\mu i, j}+g_{\mu l, i}-g_{i j, \mu}\right)$ involve only first derivatives of the metric tensor and the inverse of the metric tensor. Since we have by definition of the chart norm a positive lower bound on $|\operatorname{det} g|$ in terms of $\Theta$ and an upper bound bound on $\left\|g_{i j}\right\|_{\mathrm{C}^{2, \alpha}}$ in terms of $\Theta$ and $\varrho$, there is some upper bound on the $\mathrm{C}^{2, \alpha}$-norm of $\frac{1}{\operatorname{det} g}$ by Lemma 1.12 . Therefore there is also an upper bound on the $\mathrm{C}^{1, \alpha}$-norm of each $\Gamma_{i j}^{k}$ due to Corollary 1.11 Using the functoriality of the operation $\mathcal{P}$, Lemma 1.8 , we get the continuous map

$$
D=\mathcal{P}\left((M, p) \mapsto\left(M, p, R_{\text {Riem. }} .\right)\right): \mathcal{P} \mathrm{P}_{\mathcal{M}_{\mathrm{C}^{2, \alpha} \leq \Theta}^{d, \varrho}} \rightarrow \mathcal{P} \mathrm{T}_{\mathrm{C}^{\alpha} \leq \Theta^{\prime}}^{3,1} \underline{\mathrm{P}}_{\mathrm{C}^{\alpha} \leq \Theta}^{d, \varrho} .
$$

For any $P \in \overline{\mathfrak{M}}$ the law $D(P)$ is unimodular because for any bounded continuous test function $f$

$$
\begin{aligned}
& \int f(M, p, q, R) \mathrm{d} m_{\mathrm{L}}(D(P)) \\
& \stackrel{\text { Lemma } 1.7}{=} \iint f(M, p, q, R) \mathrm{d}\left(\mu_{M}^{\mathrm{L}}\right)_{*} \operatorname{vol}(q) \\
& \mathrm{d} \mathcal{P}\left(\left(M^{\prime}, p^{\prime}\right) \mapsto\left(M^{\prime}, p^{\prime}, R_{\text {Riem. }}\right)\right)(M, p, R) \\
& \stackrel{\text { Lemma }}{=} 1.8 \int f\left(M, p, q, R_{\text {Riem. }}\right) \mathrm{d} \operatorname{vol}_{M}(q) \mathrm{d} P(M, p) \\
& =\quad \iint f\left(M, p, q, R_{\text {Riem. }}\right) \mathrm{d}\left(m_{\mathrm{L}} P\right)(M, p, q) \\
& \stackrel{\text { Lemma }}{=}[2.3[\mathrm{v})] \int f\left(M, p, q, R_{\text {Riem. }}\right) \mathrm{d}\left(m_{\mathrm{R}} P\right)(M, p, q) \\
& =\quad \iint f\left(M, p, q, R_{\text {Riem. }}\right) \mathrm{d} \operatorname{vol}_{M}(p) \mathrm{d} P(M, q) \\
& \stackrel{\text { Lemma } 1.8}{=} \iint f(M, p, q, R) \mathrm{d}\left(\mu_{M}^{\mathrm{L}}\right)_{*} \operatorname{vol}(p) \\
& \mathrm{d} \mathcal{P}\left(\left(M, p^{\prime}\right) \mapsto\left(M^{\prime}, p^{\prime}, R_{\text {Riem. }}\right)\right)(M, q, R)
\end{aligned}
$$

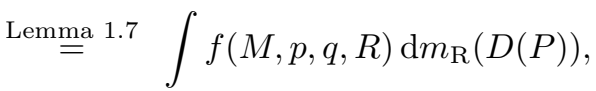

and, hence, $m_{\mathrm{L}}(D(P))$ is equal to $m_{\mathrm{R}}(D(P))$. Moreover $D(P)$ is always supported on the image of $\mathrm{P} \underset{\mathcal{M}^{2}}{\mathrm{C}^{2, \alpha} \leq \Theta}$ under the map $(M, p) \mapsto\left(M, p, R_{\text {Riem. }}\right)$., hence $D(P)$ is also a random curvature tensor.

The section property follows again by functoriality

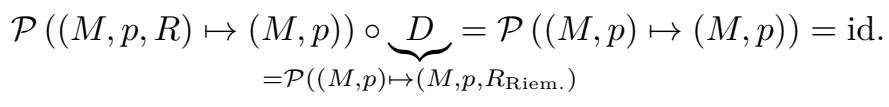

\subsubsection{A first geometric criterion}

The second criterion is geometric relying on results of Lessa [Les15] in the context of GH-convergence of smooth manifolds. The main core of his approach is the following definition, which takes all covariant derivatives of the curvature tensor into account. Such an analysis can also be refined by considering only finitely many such covariant derivatives, see Eichhorn Eic91. 
Definition 2.20 (uniformly bounded geometry). Let $r$ and $\left\{C_{m}\right\}_{m=0}^{\infty}$ be positive real constants. A smooth Riemannian manifold has uniformly bounded geometry with respect to $r$ and $\left\{C_{m}\right\}_{m=0}^{\infty}$ if

- $\operatorname{inj} M \geq r$,

- $\left\|\nabla^{m} R\right\| \leq C_{m}$ for $m=0, \ldots$ where $R=R_{\lambda \mu \nu}^{k}$ denotes the Riemannian curvature tensor, $\nabla$ denotes the covariant derivative, and we use the tensor norms induced by the Riemannian metric.

Theorem 2.21. Let $r$ and $\left\{C_{m}\right\}_{m=0}^{\infty}$ be positive real constants. Further let $\mathfrak{M}$ denote the class of compact smooth Riemannian manifold having uniformly bounded geometry with respect to $r$ and $\left\{C_{m}\right\}_{m=0}^{\infty}$. Then the parameter $\varphi_{\Pi}$ as defined in Theorem 2.18 is testable in constant time on the class $\mathfrak{M}$.

Proof. Let $\mathrm{P} \mathcal{M}_{\left\{C_{m}\right\}_{m=0}^{\infty}}^{d, r}$ denote the class of pointed smooth Riemannian manifolds having uniformly bounded geometry with respect to $r$ and $\left\{C_{m}\right\}_{m=0}^{\infty}$. For this class of spaces a Fundamental Theorem of Convergence Theory holds Les15. Theorem 3.1], i.e. on this class the $\mathrm{C}^{\infty}$-topology and the PGH-topology coincide and are compact. Therefore this class is also closed and every law in $\overline{\mathfrak{M}} \subset \mathcal{P} \mathrm{PM}$ is supported on this set. Moreover $\mathfrak{M}$ is relatively compact according to Lemma 2.8

For $m=2$ uniformly bounded geometry implies a lower bound on the Ricci curvature. Together with the lower bound on the injectivity radius this implies by a theorem of Anderson and Cheeger AC92 some $\Theta$ bound on the $\mathrm{C}^{\alpha}$-norm on some scale $\varrho>0$. Hence we can also consider $\mathfrak{M}$ as a subset of $\mathcal{P} P \mathcal{M}_{\mathrm{C}^{\alpha}<\Theta}^{d, \varrho}$. Again $\mathfrak{M}$ is relatively compact according to Lemma 2.8 and every law in the closure is supported on spaces with uniformly bounded geometry.

As in the proof of the analytic criterion, Theorem 2.19 the map $D$ is given by applying the function $\mathcal{P}$ to the map

$$
(M, p) \mapsto\left(M, p, R_{\text {Riem. }}\right)
$$

assigning to each metric space in $\mathrm{P} \mathcal{M}_{\left\{C_{m}\right\}_{m=0}^{\infty}}^{d, r}$ the Riemannian curvature tensor. Eichhorn Eic91 provided several regularity results for the regularity of normal coordinates for spaces of uniformly bounded geometry: On normal coordinates $\varphi: \mathrm{B}(x, r) \rightarrow M$ the $\mathrm{C}^{1}$-norm of the metric tensor is uniformly bounded in terms of $r, C_{0}$, and $C_{1}$ Eic91, Corolarry 2.2]. Hence we also have a bound on $\|\varphi\|_{\mathrm{C}^{\alpha}}$ for some $\alpha \in(0,1]$. In the same charts there is also a bound on the second derivative of the Christoffel symbols depending only on $r, C_{0}, \ldots, C_{3}$ [Eic91, Theorem A]. Thus in these normal charts also the $\mathrm{C}^{\alpha}$-norm of $R_{\text {Riem. }}$ is bounded. This implies that the codomain of the assignment $(M, p) \mapsto\left(M, p, R_{\mathrm{Riem}}\right)$ can be chosen as $\mathrm{T}_{\mathrm{C}^{\beta} \leq \Theta^{\prime}}^{3,1} \mathrm{P}_{\mathcal{M}_{\mathrm{C}^{\alpha} \leq \Theta}^{d, \varrho}}$ for some $\beta \in(0,1]$ and $\Theta, \Theta^{\prime}>0$.

Unimodularity and the section property are proved like in the proof of Theorem 2.19 .

\subsection{Main results}

The main theorem can be stated in an analytic and a geometric way. The latter, Theorem 2.24, is a consequence of the former. 


\subsubsection{Main theorem and lemma}

Recall from $\sqrt{1.23}$ that $\mathrm{P}_{\mathrm{M}_{\mathrm{C}}^{\alpha}<\Theta}^{d, \varrho}$ har denotes the class of pointed Riemannian manifolds with harmonic metrics tensors bounded uniformly with respect to the Hölder norm on some chart of radius $\varrho$.

Theorem 2.22 (Main Theorem). (Assuming the validity of Lemma 3.9) Let $\Lambda \in \mathbb{R}, i>0$ and $C>0$. For $d \geq 1$, let $\Pi$ be an invariant polynomial on $\mathrm{M}_{d}(\mathbb{C})$. Given $\alpha>0$ one can find $\Theta>0$ such that for all scales $\varrho \in(0,1)$ the parameter $\varphi_{\Pi}$ is testable in constant time on

$$
\left\{\begin{array}{c|c}
(M, g) \in \mathrm{P}_{\mathcal{M}^{\alpha} \leq \Theta}^{d, \varrho \text { har }} & \begin{array}{c}
M \text { is smooth, } \\
\operatorname{Ric} M \geq \Lambda, \operatorname{inj}(M, g) \geq i, \text { and } \\
\operatorname{vol}(\mathrm{B}[x, r]) \leq C^{r} \text { for all } x \in M, r \geq 0
\end{array}
\end{array}\right\} .
$$

As in the case of Theorem 2.19 the key stone of the testability criterion for $\mathrm{C}^{\alpha}$-regular metrics $(\alpha \in(0,1])$ is the Fundamental Theorem of Convergence

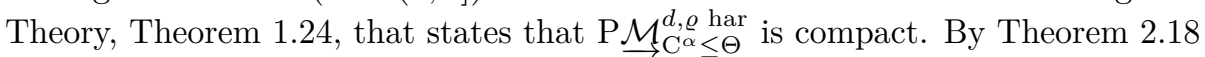
this main theorem is an immediate consequence of the following lemma:

Lemma 2.23 (Main Lemma). (Assuming the validity of Lemma 3.9) Given $\Lambda \in \mathbb{R}, i>0, C>0, d \geq 1, \alpha>\beta>0$, and a scale $\varrho \in(0,1)$, for sufficiently small $\Theta>0$ there is a continuous section

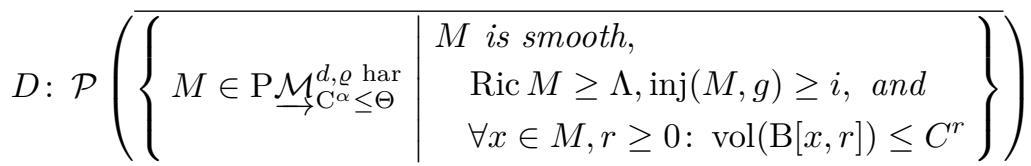

$$
\begin{aligned}
& \rightarrow \mathcal{P}\left(\mathrm{T}_{\mathrm{C}^{\beta} \leq \Theta^{\prime}}^{3,1} \stackrel{\mathrm{P}}{\rightarrow}_{\mathrm{C}^{\alpha} \leq \Theta}^{d, \varrho \text { har }}\right)
\end{aligned}
$$

for some $\Theta^{\prime}>0$ such that $D(P)$ is a unimodular curvature tensor (see Definition 2.16 and Theorem 2.18).

\subsubsection{Geometric version}

The geometric version of the main theorem relies on the following theorem that goes back to Anderson and Cheeger AC92] and can be stated in terms of chart norms as follows Pet16, Theorem 11.4.15; AC92, p. 267, Remark (2)]: Given $\alpha \in(0,1), i>0$, and $\Lambda \in \mathbb{R}$ one can find for each $\Theta>0$ a scale $\varrho=\varrho(d, \alpha, \Lambda, i)>0$ such that any pointed Riemannian $d$-manifold with

$$
\operatorname{Ric} M \geq-\Lambda, \quad \operatorname{inj} M \geq i
$$

is in $\mathrm{P}_{\mathrm{C}^{\alpha} \leq \Theta}^{d, \varrho \text { har }}$. Also remember that, by Bishop-Gromov inequality, a lower Ricci bound gives a bound $\operatorname{vol}(\mathrm{B}[x, r]) \leq C^{r}$ for any $x \in M, r \geq 0$ and some constant $C=C(d, \Lambda)$. Taking $\Theta=\Theta(d, \alpha)$ from the Main Theorem and plugging it into Anderson's theorem eventually allows us to reduce the assumptions of the Main Theorem obtaining the following geometric version:

Theorem 2.24 (Geometric Main Theorem). (Assuming the validity of Lemma 3.9) Let $\Pi$ be an invariant polynomial on $\mathrm{M}_{d}(\mathbb{C})$ and $i>0, \Lambda \in \mathbb{R}$. On the class of smooth d-dimensional oriented Riemannian manifolds $M$ satisfying 
(i) $\operatorname{Ric} M \geq-\Lambda$,

(ii) $\operatorname{inj} M \geq i$

the parameter $\varphi_{\Pi}$ is testable in constant time.

Remark 2.25. (i) As mentioned in the original publication AC92 under the above assumption the metric tensor posses even one weak derivative, that is, they are in $\mathrm{W}^{1, p}$ for $1 \leq p<\infty$. Unfortunately, GH-convergence of manifolds translates only in strong convergence on charts. Therefore the arguments from Banach spaces in Chapter 3.2.3 do not apply immediately (and probably not at all).

(ii) In the context of regularity by Ricci curvature assumptions one should also mention Anderson [And90], who proves under an additional upper Ricci curvature bound Ric $M \leq \Lambda$ that such manifolds are even in $\mathrm{P}_{\rightarrow}^{\mathcal{M}_{\mathrm{C}^{1}, \alpha}^{d, \varrho}} \underset{ }{d, \varrho}$ for $\varrho=\varrho(d, \Lambda, i, \alpha, \Theta)$, as well as a corresponding $\mathrm{W}^{2, p}$-result with $1 \leq p<\infty$. For a textbook proof see Pet16, Lemma 11.4.1].

(iii) Recent developments in so-called Cheeger-Colding-Naber theory allow to control regularity when the lower bound on the injectivity radius is replaced by a lower bound on the volume of a ball of fix radius, see Cheeger and Naber CN13, CN15]. This weakening causes singularities in PGH-limit spaces, but it is possible to maintain some degree of regularity on a part of some a-priori volume.

We call to mind a theorem from Cheeger's PhD thesis Che67 that is restated in Petersen [Pet16, Lemma 11.4.9] as follows: Given $d \geq 2$ and $v, K \geq 0$. If a compact manifold $M$ satisfies

(i) a sectional curvature bound from both sides by $K$,

(ii) $\operatorname{vol}_{M}(\mathrm{~B}[x, 1]) \geq v$ for any $x \in M$,

then $\operatorname{inj} M \geq i$ for some $i$ that depends only on $d, K$, and $v$. Together with the fact that a lower sectional curvature bound implies a lower Ricci bound we get the alternative criterion:

Corollary 2.26. For an invariant polynomial $\Pi$, the parameter $\varphi_{\Pi}$ is testable in constant time on the class of d-dimensional Riemannian manifolds $M$ satisfying

(i) the sectional curvature of $M$ is bounded from both sides,

(ii) $\operatorname{vol} \mathrm{B}(x, 1) \geq v$.

\subsubsection{Comparison theorem}

A very easy but so far unproven consequence is the following bound, that is completely independent of the notion of Benjamini-Schramm convergence. Interest is such comparison theorems goes back to Cheeger and Gromov CG85. who give a bound in terms of the volume of the $\eta$-invariant of a Riemannian $(4 d-1)$-manifold which is the boundary of another manifold of bounded sectional curvature. 
Theorem 2.27 (volume comparison). Let $\Pi$ be an invariant polynomial on $\mathrm{M}_{d}(\mathbb{C}), i>0$, and $\Lambda \in \mathbb{R}$. There is a constant $C=C(\Pi, i, \Lambda)$ such that

$$
|\Pi[M]| \leq C \operatorname{vol}(M)
$$

for any closed Riemannian d-manifold with

(i) $\operatorname{Ric} M \geq-\Lambda$,

(ii) $\operatorname{inj} M \geq i$.

Proof. Choose $\Theta>0$ and $\alpha \in(0,1)$. Choose $\varrho=\varrho(d, \alpha, \Lambda, i)>0$ such that $\|M\|_{\mathrm{C}^{\alpha} \leq \Theta}^{d, \varrho \text { har }} \leq \Theta$ (according to Pet16, Theorem 11.4.15; AC92, p. 267, Remark (2)] as quoted above). Choose a maximal collection of points $\left\{p_{i}\right\}_{i \in I}$ that is $2 e^{-\Theta-2} \varrho$-separated (i.e. any two point in the collection have distance at least $2 e^{-\Theta-2} \varrho$ ). Such a system is automatically $e^{-\Theta-1} \varrho$-covering (meaning that $\left.\left\{p_{i} \mid i \in I\right\}^{\left.e^{-\Theta-1} \varrho\right)}=M\right)$. Choose harmonic charts $\varphi_{i}:(\mathrm{B}(0, \varrho), 0) \rightarrow\left(M, p_{i}\right)$ with $\|M\|_{\mathrm{C}^{\alpha} \leq \Theta}^{\varrho} \leq \Theta$ for each $i \in I$. By the length comparison 1.20 the space $M$ is covered by the family $\left\{\varphi_{i}\left(\mathrm{~B}\left(0, e^{-1} \varrho\right)\right)\right\}_{i \in I}$. By the same inequality and the fact that $\left\{p_{i}\right\}_{i \in I}$ is $e^{-\Theta-2} \varrho$-separated, the balls $\left\{\varphi_{i}\left(\mathrm{~B}\left(0, e^{-2 \Theta-2} \varrho / 2\right)\right)\right\}_{i \in I}$ are pairwise disjoint. This gives the estimate

$$
\# I \leq \frac{\operatorname{vol}(M)}{v}
$$

for the constant $v=v\left(d, \varrho, \Theta, e^{-2 \Theta-2} \varrho / 2\right)$ from Lemma 2.17

Let $\mu_{i}$ be a corresponding smooth partition of unity, i.e. $\varphi_{i}\left(\mathrm{~B}\left(0, e^{-1} \varrho\right)\right) \subset$ $\operatorname{supp} \mu_{i} \subset \varphi_{i}(\mathrm{~B}(0, \varrho))$ for every $i \in I$. We can define another partition of unity on $M$ by choosing a smooth bump function on $b: \mathbb{R}^{d} \rightarrow[0,1]$ such that $\operatorname{supp} b \subset \mathrm{B}[0, r]$ and $\left.b\right|_{\mathrm{B}\left[0, e^{-1} \varrho\right]}=1$, and setting

$$
\tilde{\lambda}_{i}(x):=\left\{\begin{array}{ll}
b \circ \varphi_{i}^{-1} & \text { if } x \in \varphi_{i}(\mathrm{~B}(0, \varrho)) \\
0 & \text { else }
\end{array} \quad \text { and } \quad \lambda_{i}(x):=\frac{1}{\sum_{j \in I} \tilde{\lambda}_{j}(x)} \tilde{\lambda}_{i}(x)\right.
$$

as maps $M \rightarrow[0, \infty)$. Note that in the denominator at least one summand $\tilde{\lambda}_{j}(x)$ is 1 . On any chart $\varphi \in\left\{\varphi_{i}\right\}_{i \in I}$ the function $\lambda_{i}$ reads in $\varphi$-coordinates $\lambda_{i} \circ \varphi(x)=\left(\sum_{j \in I} \tilde{\lambda}_{j} \circ \varphi\right)^{-1} \cdot b \circ \varphi_{i}^{-1} \circ \varphi$. Combining the estimate on the transition functions Theorem 1.21 with Corollaries 1.11 and 1.14 and Lemmas 1.12 and 1.16 we obtain a uniform $\mathrm{C}^{2, \alpha}$-bound on the $\lambda_{i}$ 's. Using Lemma 2.15 we estimate

$$
\begin{aligned}
|\Pi[M]| & =\left|\sum_{i} \int_{\mathrm{B}(0, \varrho)} \mu_{i} \circ \varphi_{i} \cdot \Pi\left({ }^{\varphi_{i}} R_{\lambda \mu \nu}^{k}\right) \mathrm{d} x\right| \\
& \leq \sum_{i} \int_{\mathrm{B}(0, \varrho)}\left|\Pi\left({ }^{\varphi_{i}} R_{\lambda \mu \nu}^{k}\right)\right| \mathrm{d} x \\
& \leq \frac{\operatorname{vol}(M)}{v} \cdot \sup _{i \in I} \int_{\mathrm{B}(0, \varrho)}\left|\Pi\left({ }^{\varphi_{i}} R_{\lambda \mu \nu}^{k}\right)\right| \mathrm{d} x .
\end{aligned}
$$

The integrand $\Pi\left({ }^{\varphi_{i}} R_{\lambda \mu \nu}^{k}\right)$ is a polynomial of degree $d / 2$ in the ${ }^{\varphi_{i}} R_{\lambda \mu \nu}^{k}$ 's, which are defined by (2.6) and 2.8) in terms of products of the $\lambda_{i}$ 's, its first derivatives, the transition functions $\varphi_{i}^{-1} \circ \varphi_{j}(i, j \in I)$ and its first and second derivatives. Hence, by Corollaries 1.11 and 1.14 , there is a $\mathrm{C}^{0}$-bound on the integrand. Thus $\int_{\varphi_{i}(\mathrm{~B}(0, \varrho))}\left|\Pi\left({ }^{\varphi_{i}} R_{\lambda \mu \nu}^{k}\right)\right| \mathrm{d} x$ is bounded by a constant. 


\section{Chapter 3}

\section{Proof of the main lemma}

In this chapter we are going to prove Lemma 2.23 .

\subsection{Factorization of Hölder spaces}

\subsubsection{Tangent space factorization}

All Banach spaces considered will be over the real numbers. Remember that the tangent space at a point $b$ in a Banach space $\mathcal{B}$ is a copy of $\mathcal{B}$. Given an (algebraic) vector space $V$ and a vector subspaces $V_{1}$ we can always find a vector space complement $V_{2}$ such that $V=V_{1} \oplus V_{2}$ as an algebraic biproduct of vector spaces. Unfortunately, this is not true in categories of vector spaces with additional structure, like Banach spaces. In the latter case one has to hope that not only the subspace $V_{1}$ is a Banach space, i.e. is closed in $V$, but also that there is a complement that is a closed as well. The following lemmas below, Lemmas 3.1 and 3.2 establish this fact in the cases relevant to our investigation.

Let $r>0$. For the rest of this subsection we will adopt the shorthands

$$
0^{r]}:=\mathrm{B}_{\mathbb{R}^{d}}[0, r] \quad \text { and } \quad 0^{r)}:=\mathrm{B}_{\mathbb{R}^{d}}(0, r) .
$$

Further let $\vec{x}=\left(x_{1}, \ldots, x_{k}\right)$ be an ordered collection of points in $\mathbb{R}^{d}$ for $k=$ $0, \ldots$ The scaling by a factor $r$ is denoted by $r \vec{x}$. For a partially defined map $f: \mathbb{R}^{d} \rightarrow \mathbb{R}^{d}$ that is defined on $x_{1}, \ldots, x_{k}$ we define $f(\vec{x})=\left(f\left(x_{1}\right), \ldots, f\left(x_{k}\right)\right.$. We define $\mathrm{ev}_{\vec{x}}: \mathrm{C}^{1, \alpha}\left(0^{r]}, \mathbb{R}^{d}\right) \rightarrow \mathbb{R}^{k d}$ and the Banach space $\mathrm{C}^{1, \alpha}\left(0^{r]}, \mathbb{R}^{d}\right)_{\vec{x}}$ by

$$
\begin{aligned}
\mathrm{ev}_{\vec{x}}: f & \mapsto\left(f\left(x_{1}\right), \ldots, f\left(x_{k}\right)\right) \\
\mathrm{C}^{1, \alpha}\left(0^{r]}, \mathbb{R}^{d}\right)_{\vec{x}} & :=\operatorname{ker} e v_{\vec{x}} \\
& =\left\{f \in \mathrm{C}^{1, \alpha}\left(0^{r]}, \mathbb{R}^{d}\right) \mid f\left(x_{1}\right)=\ldots=f\left(x_{k}\right)=0\right\} .
\end{aligned}
$$

Further define the following spaces of matrix-valued functions

$$
\begin{aligned}
\mathrm{C}^{\alpha}\left(0^{r]}, \mathrm{Sym}^{2}\right) & :=\left\{f \in \mathrm{C}^{\alpha}\left(0^{r]}, \mathbb{R}^{d \cdot d}\right) \mid f(x) \text { is a symmeric }\right\} \text { and } \\
\mathrm{C}^{\alpha}\left(0^{r]}, \Lambda^{2}\right) & :=\left\{f \in \mathrm{C}^{\alpha}\left(0^{r]}, \mathbb{R}^{d \cdot d}\right) \mid f(x) \text { is skew-symmeric }\right\}
\end{aligned}
$$

where $x \in 0^{r]}$. Both spaces can be identified with $\mathrm{C}^{\alpha}\left(0^{r]}, \mathbb{R}^{n(n+1) / 2}\right)$ and $\mathrm{C}^{\alpha}\left(0^{r]}, \mathbb{R}^{(n-1) n / 2}\right)$ respectively. Moreover Riemannian metrics on $0^{r]}$ can be viewed as elements of $\mathrm{C}^{\alpha}\left(0^{r]}, \mathrm{Sym}^{2}\right)$. 
Recall the shorthand $f_{i, j}=\frac{\partial}{\partial x_{j}} f_{i}=\partial_{j} f_{i}$ and that $\mathrm{C}_{0}^{1}\left(0^{r]}, \mathbb{R}^{d}\right) \subset \mathrm{C}^{1}\left(0^{r]}, \mathbb{R}^{k}\right)$ denotes the space of functions with compact support inside $0^{r}$, which fails to be a Banach space. Further define the interior derivative of $\omega \in \mathrm{C}^{1, \alpha}\left(0^{r]}, \Lambda^{2}\right)$ by

$$
\delta \omega:=\left(\sum_{j=1}^{d} \omega_{j i, j}\right)_{i=1}^{d}=\sum_{i, j=1}^{d} \omega_{j i, j} \mathrm{~d} x_{i} .
$$

Moreover let the continuous dual space of a topological vector space be

$$
V^{*}=\{f: V \rightarrow \mathbb{R} \text { continuous and linear }\}
$$

with topology induced by evaluation, i.e. the coarsest topology such that every map ev $v_{v}: V^{*} \rightarrow \mathbb{R}, f \mapsto f(v)$ with $v \in V$ is continuous. We continue to define $\operatorname{grad}: \mathrm{C}^{1, \alpha}\left(B, \mathbb{R}^{d}\right) \rightarrow \mathrm{C}^{\alpha}\left(B, \mathbb{R}^{d \cdot d}\right)$ by

$$
\operatorname{grad} f=\left(\frac{\partial f}{\partial x_{1}}, \ldots, \frac{\partial f}{\partial x_{d}}\right) ;
$$

curl: $\mathrm{C}^{\alpha}\left(0^{r]}, \mathbb{R}^{d}\right) \rightarrow\left(\mathrm{C}_{0}^{1}\left(0^{r]}, \Lambda^{2}\right)\right)^{*}$ by

$$
\operatorname{curl}(v): \omega \mapsto-\int\langle v, \delta \omega\rangle,
$$

i.e. a weak form of the operator curl: $v \mapsto\left(v_{j, i}-v_{i, j}\right) \mathrm{d} x_{i} \wedge \mathrm{d} x_{j}$ obtained from integration by parts CDK11, Theorem 3.28]; $\operatorname{curl}^{*}: \mathrm{C}^{1, \alpha}\left(0^{r]}, \Lambda^{2}\right) \rightarrow \mathrm{C}^{\alpha}\left(0^{r]}, \mathbb{R}^{d}\right)$ by

$$
\operatorname{curl}^{*}(v)=\left(\sum_{j=1}^{i-1} v_{j i, j}-\sum_{j=i+1}^{n} v_{i j, j}\right)_{i=1}^{n}
$$

and, finally, div: $\mathrm{C}^{\alpha}\left(0^{r]}, \mathbb{R}^{d}\right) \rightarrow\left(\mathrm{C}_{0}^{1}\left(0^{r]}, \mathbb{R}\right)\right)^{*}$ by

$$
\operatorname{div}(u): \varphi \mapsto-\int \sum_{i} u_{i} \partial_{i} \varphi
$$

Lemma 3.1. Let $\alpha \in(0,1), s>r \geq 0$, and $\vec{x} \in\left(0^{s]} \backslash 0^{r]}\right)^{k}$. The following sequences are right exact in the category of Banach spaces and bounded linear maps. Moreover the sequences split, i.e. each middle object decomposes into a direct sum of the right space and some complement. The sequences are:

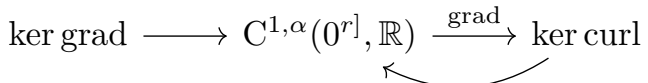

$$
\begin{aligned}
& \operatorname{ker}\left(\left.f \mapsto f\right|_{\mathrm{B}[0, r]}\right) \longrightarrow \mathrm{C}^{\alpha}\left(0^{s]}, \mathbb{R}^{d}\right)_{\vec{x}} \stackrel{\left.f \mapsto f\right|_{\mathrm{B}[0, r]}}{\longrightarrow} \mathrm{C}^{\alpha}\left(0^{r]}, \mathbb{R}^{d}\right) \\
& \mathrm{C}^{1, \alpha}\left(0^{r]}, \mathbb{R}^{d}\right)_{\vec{x}} \longrightarrow \mathrm{C}^{1, \alpha}\left(0^{r]}, \mathbb{R}^{d}\right) \stackrel{\mathrm{ev} \vec{x}}{\longrightarrow} \mathbb{R}^{k d} .
\end{aligned}
$$


Proof. Obviously, all maps going from left to right in the diagrams are linear and continuous. Moreover, curl is linear, and also continuous as well since given $v_{n} \rightarrow 0$ in $\mathrm{C}^{\alpha}\left(0^{r]}, \mathbb{R}^{d}\right)$ implies $-\int\langle v, \delta \omega\rangle \rightarrow 0$ for any $\omega \in \mathrm{C}_{0}^{1}\left(0^{r]}, \Lambda^{2}\right)$ by the Dominated Convergence Theorem. As kernels of continuous maps are closed, all spaces in the sequences are Banach spaces.

By general yoga of topological vector spaces Sch71, § I.2.2] it is sufficient to show that the right map is surjective and it admits a bounded linear split. In case of $3.2 \mathrm{a}$ this is a PDE problem, that is solved by CDK11, Theorem 8.3] (consider also Remarks 8.5 (ii), (iv), and (v) therein). In case $(3.2 \mathrm{~b}$ ) the question translates into an extension problem which is solved in CDK11, Theorem 16.11; GT77, Lemma 6.37]. For the remaining case (3.2c) choose pairwise disjoint neighborhoods of $x_{1}, \ldots, x_{k}$ and take smooth bump functions $b_{1}, \ldots, b_{k}$ of these neighborhoods with $b_{1}\left(x_{1}\right)=\ldots=b_{k}\left(x_{k}\right)=1$. A split is then given by $f \mapsto f\left(x_{1}\right) b_{1}+\ldots+f\left(x_{k}\right) b_{k}$.

In the original version of the thesis there was a mistake in the proof of the following lemma which could not be remedied, see the remark preceding this thesis.

Lemma 3.2. (No proof of this lemma currently known, see the remark preceding this thesis) Let $\alpha \in(0,1)$. The following sequence is left exact in the category of topological vector spaces. Moreover the sequence splits in the category of Banach spaces, i.e. the left and the middle objects are Banach spaces and the middle space decomposes as Banach spaces into a direct sum of the former space and some complement. The sequence is:

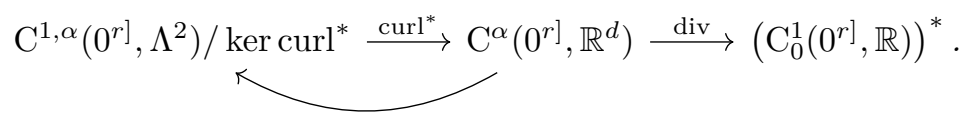

Remark 3.3. The theorem used by the preceding lemmas, namely CDK11, Theorem 8.3], holds for higher degrees of Hölder regularity $\mathrm{C}^{m, \alpha}$ with $\alpha \in(0,1)$. But it is in general not known in the cases $\alpha=0$ or $\alpha=1$ and false is specific instances of these cases DFT03, Pre97 - though no counterexample in the instances relevant for the lemma is known to the author. Hence from this moment onward we have to assume $\alpha \in(0,1)$.

\subsubsection{The implicit function theorem}

By a locally defined map between two pointed spaces we mean a map that preserves the base point and is defined on a neighborhood of the base point of its domain. This domain is called domain of definition. When writing $A \oplus B$, we consider this space pointed at the origin, i.e. $A \oplus B=(A \oplus B, 0)$. Recall that the Fréchet derivative of a locally defined map $A:(\mathcal{E}, e) \rightarrow(\mathcal{F}, f)$ at $e \in \mathcal{E}$ is, if it exists, the bounded linear map given by $\left(D_{e} A\right)(v):=\lim _{\tau \rightarrow 0} \frac{A(e+\tau v)-A e}{\tau v}$. A local

Fréchet isomorphism is a locally defined continuously Fréchet differentiable map with a locally defined continuously Fréchet differentiable inverse. The domain of definition of the inverse is called codomain. We state the local submersion theorem Lan12, Corollary 5.7]: 
Lemma 3.4 (local submersion theorem). Consider a locally defined continuously Fréchet differentiable map

$$
A:\left(\mathcal{E}, e^{0}\right) \rightarrow\left(\mathcal{F}, f^{0}\right)
$$

between pointed Banach spaces. Assume that the differential $D_{e^{0}} A$ is surjective and its kernel has a closed complement. Then there is a local Fréchet isomorphisms (up to restriction of the domains of definition) $\varphi:\left(\mathcal{E}_{1} \oplus \mathcal{E}_{2}, 0\right) \rightarrow\left(\mathcal{E}, e^{0}\right)$ such that

$$
A \circ \varphi\left(e_{1}, e_{2}\right)=A \circ \varphi\left(e_{1}, 0\right)
$$

for all $\left(e_{1}, e_{2}\right)$ in the domain of definition of $\varphi$. Moreover $\varphi$ is of the form

$$
\varphi\left(e_{1}, e_{2}\right)=e_{1}+\varphi_{2}\left(e_{1}, e_{2}\right)
$$

with $\varphi_{2}$ valued in $\mathcal{E}_{1}$.

Related to the local submersion theorem is the implicit function theorem. A nice generalization thereof was given by Robinson [Rob91. Corollary 3.4]. The instance crucial to our investigation reads as follows:

Lemma 3.5. Let $\mathcal{Y}$ be a Banach space and $\mathcal{X}, \mathcal{Z}$ be normed vector spaces. Let $x^{0}, y^{0}$ be points in $\mathcal{X}, \mathcal{Y}$ respectively, and let $\mathcal{U}$ a neighborhood of $x^{0}$ and $\mathcal{V}$ a neighborhood of $y^{0}$. Suppose $F$ is a function from $\mathcal{U} \times \mathcal{V}$ to $\mathcal{Z}$ with $F\left(x^{0}, y^{0}\right)=0$, and is Fréchet differentiable at $\left(x^{0}, y^{0}\right)$. Suppose further:

(i) $F(., y)$ is Lipschitz admitting a uniform Lipschitz constant for all $y \in \mathcal{Y}$;

(ii) the derivative at $\left(x^{0}, y^{0}\right)$ in $y$-direction $D_{\left(x^{0}, y^{0}\right)} F \mid \mathcal{Y}$ is strong, meaning that for the first order approximation $T_{1}:=F\left(x^{0}, y^{0}\right)+\left.D_{\left(x^{0}, y^{0}\right)} F\right|_{\mathcal{Y}}: \mathcal{Y} \rightarrow \mathcal{Z}$ for every $\varepsilon>0$ the following error estimate holds on a sufficiently small neighborhood of $\left(x^{0}, y^{0}\right)$ :

$$
\left|\left(F(x, y)-T_{1}(y)\right)-\left(F\left(x, y^{\prime}\right)-T_{1}\left(y^{\prime}\right)\right)\right| \leq \varepsilon\left|y-y^{\prime}\right| ;
$$

(iii) $\left.D_{\left(x^{0}, y^{0}\right)} F\right|_{\mathcal{Y}}$ is bijective.

Then there are neighborhoods $\mathcal{U}^{\prime}$ of $x^{0}$ and $\mathcal{V}^{\prime}$ of $y^{0}$, and a function $P: \mathcal{U}^{\prime} \rightarrow \mathcal{V}^{\prime}$ such that

(i) $P\left(x^{0}\right)=y^{0}$;

(ii) $P$ is Lipschitz;

(iii) for each $x \in \mathcal{U}^{\prime}, P(x)$ is the unique solution in $\mathcal{V}^{\prime}$ of $F(x, y)=0$.

Lemma 3.6. Let $\varrho>0, m \geq 0$, and $\alpha \in[0,1]$ with $m+\alpha \geq 1$. Set $B:=\mathrm{B}[0, \varrho]$. For a neighborhood $\mathcal{U}^{\prime}$ of $i d \in \mathrm{C}^{m, \alpha}\left(B, \mathbb{R}^{d}\right)$, id: $x \mapsto x$, every function $f \in \mathcal{U}$ with values in $B$ has a unique inverse $f^{-1}$, i.e. $f^{-1} \circ f=f \circ f^{-1}=i d$. This inverse $f^{-1}$ depends continuously on such $f$ 's.

Proof. Set $\|\|:.=\|\cdot\|_{\mathrm{C}^{m, \alpha}} ; \partial B$ denotes the boundary of $B$. The idea is to apply Lemma 3.5 to the case $\mathcal{X}=\mathcal{Y}=\mathcal{Z}=\left\{f \in \mathrm{C}^{m, \alpha}\left(B, \mathbb{R}^{d}\right)|f|_{\partial B}=0\right\}$ and $\mathcal{U}=\mathcal{V}=\{f \mid\|f-i d\|<1\}$. Note that, being defined as the kernel of the restriction map to $\partial B, \mathcal{Y}$ is again a Banach space. Since $m+\alpha \geq 1$ every $f \in \mathcal{U}$ 
is at least Lipschitz with Lipschitz constant smaller than 1 . Hence for very $x \in B$ the distance $\mathrm{d}_{\mathrm{H}}((i d+f)(x)-x, \partial B)<\mathrm{d}_{\mathrm{H}}(x, \partial B)$. Thus $(i d+f)(x) \in B$. Hence the map

$$
F: \mathcal{U} \times \mathcal{V} \rightarrow \mathcal{Z},(f, g) \mapsto(i d+g) \circ(i d+f)-i d
$$

is well defined.

Obviously, $F(0,0)=0$. At $(0,0)$ the Fréchet derivative is calculated using infinitesimal elements $f_{\delta}$ and $g_{\delta}$ :

$$
\begin{aligned}
\left(\left(i d+g_{\delta}\right) \circ\left(i d+f_{\delta}\right)-i d\right)-(i d \circ i d-i d) \\
\quad=f_{\delta}+g_{\delta} \circ\left(i d+f_{\delta}\right) \\
\quad=f_{\delta}+g_{\delta}+\left(g_{\delta} \circ\left(i d+f_{\delta}\right)-g_{\delta} \circ i d\right)
\end{aligned}
$$

Lemma 1.17 indicates that the last summand vanishes at rate $\left\|g_{\delta}\right\|^{2}$. Hence

$$
=f_{\delta}+g_{\delta} .
$$

Thus $D_{(0,0) F}=\mathrm{id}_{\mathcal{X}}+\mathrm{id}_{\mathcal{Y}}$. We continue to check the remaining assumptions of Lemma 3.5

(i) For $g \in \mathcal{V}$ and $f_{1}, f_{2} \in \mathcal{U}$ we obtain by Lemma 1.17

$$
\begin{aligned}
\left\|F\left(f_{1}, g\right)-F\left(f_{2}, g\right)\right\| & \leq\left\|g \circ f_{1}-g \circ f_{2}\right\| \\
& \leq C\|g\|\left(1+\left\|f_{1}\right\|+\left\|f_{2}\right\|\right)\left\|f_{1}-f_{2}\right\| \\
& \leq C(1+2)\left\|f_{1}-f_{2}\right\| .
\end{aligned}
$$

Thus there is a Lipschitz bound on $F(., g)$ uniform in $g$.

(ii) Note that $D_{F(0,0)} \mid \mathcal{Y}=\operatorname{id} \mathcal{Y}$. For $T_{1}=F(0,0)+D_{F(0,0)} \mid \mathcal{Y}=0+\operatorname{id} \mathcal{Y}$ we estimate using again Lemma 1.17

$$
\begin{aligned}
& \left\|\left(F(f, g)-T_{1}(g)\right)-\left(F\left(f, g^{\prime}\right)-T_{1}\left(g^{\prime}\right)\right)\right\| \\
& \quad=\left\|((i d+g) \circ(i d+f)-i d-g)-\left(\left(i d+g^{\prime}\right) \circ(i d+f)-i d-g^{\prime}\right)\right\| \\
& \quad=\left\|\left(g-g^{\prime}\right) \circ(i d+f)+\left(g-g^{\prime}\right) \circ i d\right\| \\
& \quad \leq C\left\|g-g^{\prime}\right\|(1+\|i d+f\|+\|i d\|)\|f\| \\
& \quad \leq 3 C\|f\|\left\|g-g^{\prime}\right\| .
\end{aligned}
$$

Hence shrinking the domain of $f$ sufficiently, we get a Lipschitz estimate in $g$ with arbitrarily small Lipschitz constant.

(iii) Since $D_{(0,0) F}\left|\mathcal{Y}=\operatorname{id}_{\mathcal{Y}}, D_{(0,0) F}\right| \mathcal{Y}$ is bijective.

Hence Lemma 3.5 is applicable. But the conclusion of this lemma is exactly our claim.

\subsubsection{Geometric applications}

Let $r>0$. We identify the tangent bundle $\mathrm{T} 0^{r}$ on the ball $0^{r}$ with $0^{r]} \times$ $\mathbb{R}^{d \cdot d} \simeq 0^{r]} \times \mathbb{R}^{d \cdot d}$ canonically. By this identification a bundle isomorphism $\varphi: \mathrm{T}^{r]} \rightarrow \mathrm{T}^{r]}$ (with $\varphi(x, v)=(x, \tilde{\varphi}(v))$ ) can be identified with a matrix valued function $0^{r]} \rightarrow \mathbb{R}^{d \cdot d}$. By an orthonormal $\mathrm{C}^{\alpha}$-frame for $g$ we denote a bundle isomorphism $x \mapsto B_{x}$ that is in $\mathrm{C}^{\alpha}\left(0^{r]}, \mathbb{R}^{d \cdot d}\right)$ and maps the canonical Euclidean base of $\mathbb{R}^{d}$ to base that is orthonormal with respect to $g$. 
Lemma 3.7. Let $g$ be a Riemannian $\mathrm{C}^{\alpha}$-metric on some ball $\mathrm{B}[0, r]$. Then $g$ admits an orthonormal $\mathrm{C}^{\alpha}$-frame $B_{(.)}$. Moreover, the inverse $x \mapsto B_{x}^{-1}$ is also in $\mathrm{C}^{\alpha}\left(0^{r]}, \mathbb{R}^{d \cdot d}\right)$.

Proof. Lemma 1.12 states in our case that given a matrix valued function $A \in$ $\mathrm{C}^{1, \alpha}\left(0^{r]}, \mathbb{R}^{d \cdot d}\right)$ such that $(\operatorname{det} A)^{-1}$ is bound in absolute value, the inverse matrix function $A^{-1}$ is $\mathrm{C}^{\alpha}$-bounded by $C$, where $C=C\left(\|A\|_{\mathrm{C}^{0}}, r\right)$. An immediate application of this statement is that for any vector valued function $f \in \mathrm{C}^{\alpha}\left(0^{r]}, \mathbb{R}^{d}\right)$ with $0<C_{1} \leq|f| \leq C_{2}$ the reciprocal norm $\frac{1}{|f|}$ is bounded in the same way.

Let $\left(x, e_{1}\right),\left(x, e_{2}\right), \ldots,\left(x, e_{n}\right)$ be the Euclidean standard frame of $\mathrm{T} 0^{r]}$. We calculate $B$ by the Gram-Schmidt process, i.e.

$$
\begin{aligned}
\tilde{B}_{x, e_{1}} & :=\left(x, e_{1}\right) \\
\tilde{B}_{x, e_{2}} & :=\left(x, e_{2}\right)-\frac{\left\langle e_{2}, \tilde{B}_{x, e_{1}}\right\rangle_{g}}{\left|\tilde{B}_{x, e_{1}}\right|_{g}^{2}} \tilde{B}_{x, e_{1}} \\
\vdots & \vdots \\
\tilde{B}_{x, e_{d}} & \vdots=
\end{aligned}
$$

and

$$
B_{x, e_{1}}:=\frac{\tilde{B}_{x, e_{1}}}{\left|\tilde{B}_{x, e_{1}}\right|_{g}}, \quad \ldots, \quad B_{x, e_{d}}:=\frac{\tilde{B}_{x, e_{d}}}{\left|\tilde{B}_{x, e_{d}}\right|_{g}} .
$$

We have to check inductively that the field defined in each step remains $\mathrm{C}^{\alpha}$-bounded. Each step involves only $\mathrm{C}^{\alpha}$-functions (assuming that all functions defined before are of class $\mathrm{C}^{\alpha}$ ) and operations that are either of the form $f \mapsto \frac{1}{|f|}$ or addition and multiplication (which are $\mathrm{C}^{\alpha}$-bounded). As the Gram-Schmidt process is well-defined (i.e. denominators do not vanish), all reciprocal norms involved in the process are bounded uniformly since $0^{r]}$ is compact. Hence by the first paragraph the frame map $B=\left(B_{., e_{1}}, \ldots, B_{., e_{d}}\right)$ is $\mathrm{C}^{\alpha}$-bounded.

For the inverse frame map apply directly the statement of [CDK11] from the first paragraph. This gives that $B_{x}^{-1}$ is in $\mathrm{C}^{\alpha}\left(0^{r]} \times \mathbb{R}^{d \cdot d}, 0^{r]} \times \mathbb{R}^{d \cdot d}\right)$.

Lemma 3.8. Let $B \subsetneq B^{\prime}$ be two Euclidean balls. The pull-back map

$$
\begin{aligned}
(.)^{*}(.): \mathrm{C}^{\alpha}\left(B^{\prime}, \operatorname{Sym}^{2}\right) \times\left(i d+\mathrm{C}^{1, \alpha}\left(B, \mathbb{R}^{d}\right)_{\vec{x}}\right) & \rightarrow \mathrm{C}^{\alpha}\left(B, \operatorname{Sym}^{2}\right), \\
(g, f) & \mapsto f^{*} g
\end{aligned}
$$

is continuously Fréchet differentiable. Moreover for any Riemannian metric $g^{0}$ the Fréchet differential at $\left(g^{0}, i d\right)$ has a factorization of the form

$$
\left(\begin{array}{cccc}
\operatorname{pr}_{G_{B 1}} & 0 & S & 0 \\
\operatorname{pr}_{G_{B 2}} & 0 & 0 & 0
\end{array}\right): \mathcal{G}_{B} \oplus \mathcal{G}_{B^{\prime} \backslash B} \oplus \mathcal{F}_{1} \oplus \mathcal{F}_{2} \rightarrow \mathcal{G}_{B 1} \oplus \mathcal{G}_{B 2},
$$

where $S$ is a linear homeomorphism and we have linear homeomorphisms

$$
\begin{aligned}
\mathcal{G}_{B} & \simeq \mathrm{C}^{\alpha}\left(B, \mathrm{Sym}^{2}\right), \quad \mathcal{G}_{B} \oplus \mathcal{G}_{B^{\prime} \backslash B} \simeq \mathrm{C}^{\alpha}\left(B^{\prime}, \mathrm{Sym}^{2}\right), \\
\mathcal{G}_{B} & \simeq \mathcal{G}_{B 1} \oplus \mathcal{G}_{B 2}, \\
\mathcal{F}_{1} \oplus \mathcal{F}_{2} & \simeq \mathrm{C}^{1, \alpha}\left(B, \mathbb{R}^{d}\right)_{\vec{x}} .
\end{aligned}
$$


Proof. We calculate the Fréchet derivative of $(.)^{*}($.$) at \left(g^{0}, f^{0}\right)$ in the direction of some tangent vector $(f, g)$, i.e. a map between tangent spaces,

$$
D_{g^{0}, f^{0}}\left((.)^{*}(.)\right): \mathrm{C}^{\alpha}\left(B^{\prime}, \operatorname{Sym}^{2}\right) \times \mathrm{C}^{1, \alpha}\left(B, \mathbb{R}^{d}\right)_{\vec{x}} \rightarrow \mathrm{C}^{\alpha}\left(B, \operatorname{Sym}^{2}\right) .
$$

Since by linearity

$$
\begin{aligned}
D_{g^{0}, f^{0}}\left((.)^{*}(.)\right)(g, f) & =\lim _{\tau \rightarrow 0} \frac{\left(f^{0}+\tau f\right)^{*}\left(g^{0}+\tau g\right)-f^{0 *} g^{0}}{\tau} \\
& =\lim _{\tau \rightarrow 0} \frac{\left(f^{0}+\tau f\right)^{*} g^{0}-f^{0 *} g^{0}}{\tau}+f^{0 *} g
\end{aligned}
$$

we have

$$
D_{g^{0}, f^{0}}\left((.)^{*}(.)\right)(f, g)=D_{f^{0}}\left(f^{*} g^{0}\right)+f^{0 *} g .
$$

This expression is continuous in $g$ and $f$ for fix $g^{0}$ and $f^{0}$. Hence pullback map has a Fréchet derivative at $\left(g^{0}, f^{0}\right)$. Since this expression is continuous in $g^{0}$ and $f^{0}$ as well, the pullback map is continuously differentiable. For the factorization we restrict to the case $\left(g^{0}, f^{0}\right)=\left(g^{0}, i d\right)$, i.e.

$$
D_{g^{0}, i d}\left((.)^{*}(.)\right)=D_{i d}\left((.)^{*} g^{0}\right)+\left(\left.g \mapsto g\right|_{\mathrm{B}[0, a]}\right) .
$$

In case of the first summand of (3.4), we calculate using the fiber-wise inverse $A_{x}: \mathrm{T}_{x}^{d} B \rightarrow B \times \mathbb{R}^{d}$ of an orthonormal frame $B_{x}: B \times \mathbb{R}^{d} \rightarrow \mathrm{T}_{x}^{d} B$ as provided by Lemma 3.7

$$
\begin{aligned}
D_{i d}\left((.)^{*} g^{0}\right)(f) & =\lim _{\tau \rightarrow 0} \frac{(i d+\tau f)^{*} g^{0}-i d^{*} g^{0}}{\tau} \\
& =\lim _{\tau \rightarrow 0} \frac{\left(\left\langle(i d+\tau f)_{*} \partial_{i},(i d+\tau f)_{*} \partial_{j}\right\rangle_{g^{0}}-\left\langle i d_{*} \partial_{i}, i d_{*} \partial_{j}\right\rangle_{g^{0}}\right)_{i j}}{\tau} \\
& =\left(\left\langle i d_{*} \partial_{i}, f_{*} \partial_{j}\right\rangle_{g^{0}}+\left\langle i d_{*} \partial_{j}, f_{*} \partial_{i}\right\rangle_{g^{0}}\right)_{i j} \\
& =\left(\left\langle A^{-1} i d_{*} \partial_{i}, A^{-1} f_{*} \partial_{j}\right\rangle_{\text {Eucl. }}+\left\langle A^{-1} i d_{*} \partial_{j}, A^{-1} f_{*} \partial_{i}\right\rangle_{\text {Eucl. }}\right)_{i j} \\
& =x \mapsto\left(\left(A_{x}^{T} A_{x}(\operatorname{grad} f)(x)\right)_{i j}+\left(A_{x}^{T} A_{x}(\operatorname{grad} f)(x)\right)_{j i}\right)_{i j} .
\end{aligned}
$$

By this base change we can fiber-wise factorize $D_{i d}\left((.)^{*} g^{0}\right)$ by bounded linear maps

$$
\begin{aligned}
\mathrm{C}^{1, \alpha}\left(B, \mathbb{R}^{d}\right) \stackrel{J}{\longrightarrow} \mathrm{C}^{\alpha}\left(B, \mathbb{R}^{d \cdot d}\right) \longrightarrow \mathrm{C}^{\alpha}\left(B, \mathbb{R}^{d \cdot d}\right) \longrightarrow \mathrm{C}^{\alpha}\left(B, \mathbb{R}^{\left(\begin{array}{l}
d \\
2
\end{array}\right)}\right) \\
h \longmapsto h_{i, j} \longmapsto A_{x}^{T} A_{x}\left(h_{i, j}\right)_{i j}=: \tilde{h}_{i j} \longmapsto\left(\tilde{h}_{i j}+\tilde{h}_{j i}\right)_{i \leq j}
\end{aligned}
$$

of Banach spaces, where $J=\operatorname{grad}^{d}$ is the Jacobi map. It has a kernel-image factorization due to 3.2a of Lemma 3.1 The bounded and linear transformation $h_{i j} \mapsto A_{x}^{T} A_{x}\left(h_{i, j}\right)_{i j}$ is invertible and therefore a linear homeomorphisms of Banach spaces. Finally, the map $\left(\tilde{h}_{i j}\right) \mapsto\left(\tilde{h}_{i j}+\tilde{h}_{j i}\right)_{i \leq j}$ has a kernel-image factorization as it is described fiber-wise by a constant matrix. This gives the desired kernel-image factorization $D_{i d}\left((.)^{*} g^{0}\right): \mathcal{F}_{1} \oplus \mathcal{F}_{2} \rightarrow \mathcal{G}_{B 1} \oplus \mathcal{G}_{B 2}$.

In case of the second summand of (3.4), there is a kernel-image factorization $\mathcal{G}_{B} \oplus \mathcal{G}_{B^{\prime} \backslash B} \simeq \mathrm{C}^{\alpha}\left(B^{\prime}, \mathrm{Sym}^{2}\right)$ with $\mathcal{G}_{B} \simeq \mathrm{C}^{\alpha}\left(B, \operatorname{Sym}^{2}\right)$ due to $3.2 \mathrm{~b}$ of Lemma 3.1 
Lemma 3.9. (The proof of this lemma is not correct and, currently, no valid proof is known, see the remark preceding this thesis) Assume $d \geq 3$. Let $g^{0} \in$ $\mathrm{C}^{\alpha}\left(0^{r]}, \mathrm{Sym}^{2}\right)$ be a harmonic metric. There is a local Fréchet isomorphism

$$
\varphi_{\text {har }}:\left(\mathcal{V}^{\text {har }} \oplus \mathcal{E}^{\text {har }}, 0 \oplus 0\right) \rightarrow\left(\mathrm{C}^{\alpha}\left(0^{r]}, \operatorname{Sym}^{2}\right), g^{0}\right)
$$

for some Banach spaces $\mathcal{V}^{\text {har }}$ and $\mathcal{E}^{\text {har }}$ such that the Fréchet derivative $D_{g^{\circ}} \varphi_{\text {har }}$ is an isomorphism and a metric $g$ in the codomain of definition of $\varphi_{\text {har }}$ is harmonic if and only if $\operatorname{pr}_{\mathcal{E}^{\text {har }}} \varphi_{\text {har }}^{-1}(g)=0$.

Proof. Recall from (1.22) that harmonicity of a $\mathrm{C}^{\alpha}$-metric means that the distributions $\Delta_{g}^{\mathrm{w}} x_{k}=\sum_{i}\left(g^{1 / 2} g^{i k}\right) \partial_{i}$ for all coordinate functions $x_{k}=x_{1}, \ldots, x_{d}$ vanish. We factorize the map $\left(\Delta_{(.)}^{\mathrm{w}} x_{1}, \ldots, \Delta_{(.)}^{\mathrm{w}} x_{d}\right)$, as follows

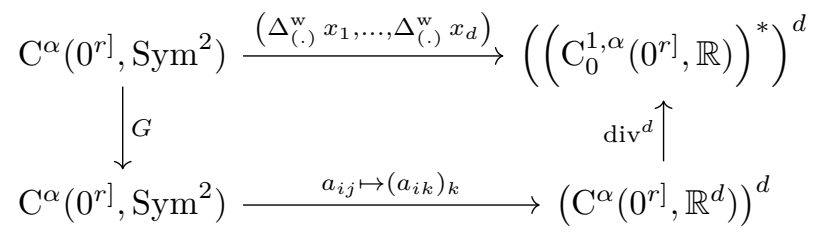

where $G: g \mapsto g^{1 / 2} g^{*}\left(g^{*}\right.$ denotes the inverse of $g .$. , which is again a symmetric matrix). The map $G$ has an inverse given by $a_{\text {.. }} \mapsto a^{1 / d-2} a^{*}$ as

$$
\left|g^{1 / 2} g^{*}\right|^{1 / d-2}\left(g^{1 / 2} g^{*}\right)^{-1}=\left(g^{d / 2}\left|g^{*}\right|\right)^{1 / d-2} g^{-1 / 2}\left(g^{*}\right)^{-1}=\left(g^{d / 2-1}\right)^{1 / d-2} g^{-1 / 2} g_{. .}=g_{.} .
$$

where $\left|a_{. .}\right|=\operatorname{det} a_{\ldots}$. To see that $G$ admits a Fréchet derivative at any point $g$ around $g^{0}$ apply basic rules of Fréchet derivatives [Die69, § 8.2] to a tangent vector $h_{. .} \in \mathrm{C}^{\alpha}\left(0^{r]}, \mathrm{Sym}^{2}\right)$

$$
\begin{aligned}
\left(D_{g} G\right) h_{. .} & =\left(\left(D_{g} g^{1 / 2}\right) h_{. .}\right) \cdot g^{*}+g^{1 / 2}\left(D_{g} g^{*}\right) h_{. .} \\
& =\frac{1}{2} g^{-1 / 2}\left(\left(D_{g} \operatorname{det} g_{. .}\right) h_{. .}\right) \cdot g^{*}+g^{1 / 2}\left(D_{g} g^{*}\right) h_{. .}
\end{aligned}
$$

provided that $g \geq \varepsilon>0$ which can be assumed by restricting to a sufficiently small neighborhood around $g^{0}$. Moreover observe that

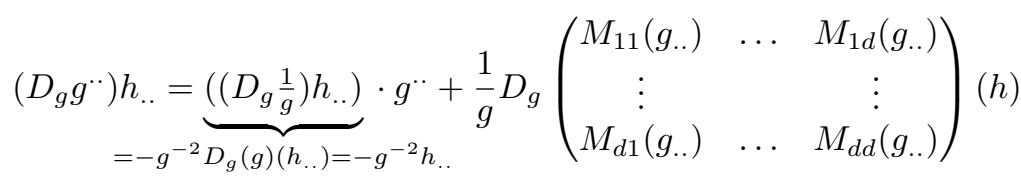

where $M_{i j}(g .$.$) denotes the i j$-minor which are determinants. As determinants are simply polynomials the Fréchet derivative of $G$ exists. By a similar argument the Fréchet derivative of the inverse of $G$ exists. Since both maps are inverse to each other their derivatives have to be invertible. This is to say that $G$ is a local Fréchet isomorphism.

The linear map $a_{i j} \mapsto\left(a_{i k}\right)_{i}$ is described fiber-wise by a constant matrix and is injective. Hence it is onto a closed linear subspace $\mathcal{F}_{1}$ of $\left(\mathrm{C}^{\alpha}\left(0^{r]}, \mathbb{R}^{d}\right)\right)^{d}$ with a closed linear complement $\mathcal{F}_{2}$. Identify $\mathcal{F}_{1}$ with $\mathrm{C}^{\alpha}\left(0^{r]}, \mathrm{Sym}^{2}\right)$. Due to $\left.3.2 \mathrm{~d}\right)$ the map $\operatorname{div}^{d}$ is onto. According to $\sqrt{3.2 \mathrm{~d}}$ of Lemma 3.2 there is a factorization of $\mathrm{C}^{\alpha}\left(0^{r]}, \mathbb{R}^{d}\right)$ into the kernel of div, let's denote it by $\mathcal{E}_{2}$ and a closed linear complement $\mathcal{E}_{1}$. This factorization applied to every factor gives a factorization 
of $\left(\mathrm{C}^{\alpha}\left(0^{r]}, \mathbb{R}^{d}\right)\right)^{d}$ into a kernel, let's call it $\tilde{\mathcal{V}}^{\text {har }}$, and a closed complement, let's call it $\tilde{\mathcal{E}}^{\text {har }}$. The harmonic metrics by $\mathcal{V}^{\text {har }}:=\tilde{\mathcal{V}}^{\text {har }} \cap \mathrm{C}^{\alpha}\left(0^{r]}, \mathrm{Sym}^{2}\right)$.

Unfortunately, by a mistake in the original version of this thesis it was assumed that the intersection $\mathcal{V}^{\text {har }}$ admits a closed complement as well. But this is not generally the case in Banach spaces, see the remark preceding this thesis. We can apply Lemma 3.4 to the map $A$ obtaining the local Fréchet isomorphism $\varphi_{\text {har }}$ we sought.

\subsection{Controlling coordinate charts}

\subsubsection{Strainers}

Strainers are a way of introducing coordinates that works generally in complete length spaces of curvature bounded from below. We resort to this concept, as it allows to introduce a kind of coordinate system canonically up to the discrete choice of an origin and $2 d$ points around it in any metric space. Note, that the standard canonical coordinate system in Riemannian geometry, normal coordinates, is ill-defined in our set-up as the geodesic equations require at least a $\mathrm{C}^{1,1}$-metric tensor to have a unique solution guaranteed by the Picard-Lindelöf theorem, see also KSS13 for the regularity of the resulting coordinate system. A counterexamples regarding the uniqueness of geodesics for $\mathrm{C}^{1, \alpha}$-metrics with $\alpha<1$ is found in [Chr91, Appendix F].

A complete introduction of the concept of strainers in metric spaces is given in Burago, Burago, and Ivanov BBI01. § 10.8.2]. For our purposes the following definition is convenient:

Definition 3.10 ( $\varepsilon$-strainer). A collection $\vec{z}=\left(z_{1}^{+}, \ldots, z_{d}^{+}, z_{1}^{-}, \ldots, z_{d}^{-}\right)$of points in Riemannian $d$-manifold $M$ is an $\varepsilon$-strainer at some point $x$ if

$$
\begin{aligned}
\left|\widetilde{\measuredangle} z_{i}^{+} x z_{j}^{+}-\frac{\pi}{2}\right| & \leq \varepsilon, \\
\left|\widetilde{\measuredangle} z_{i}^{+} x z_{i}^{-}-\pi\right| & \leq \varepsilon
\end{aligned}
$$

for $i, j \in\{1, \ldots d\}$ with $i \neq j$.

Remark 3.11. As strainer can be regarded as a coordinate system in the following sense: For an $\varepsilon$-strainer distance coordinates str: $U \rightarrow \mathbb{R}^{d}$ are given by the function

$$
\operatorname{str}(x)=\left(\left|x z_{1}\right|, \ldots,\left|x z_{d}\right|\right) .
$$

However, we will not use this definition, but only the fact stated below by Theorem 3.15 that strainers fix a choice of automorphism. The reason is that, since we assume only a lower Ricci bound, strainer coordinates do not give a local bijection. To the knowledge of the author there has not been any usage of strainers in this set-up.

There is a twofold difference between our definition and the definition given by Burago, Burago, and Ivanov BBI01. First, strict inequality is required. In our set-up weak inequality is more natural as it is stable under limits. Second, the authors also consider the case where $d$ does not equal the dimension of the space (that does not need to have an integer dimension in the classical sense anyway). Moreover their lower curvature requirement for the regularity of strainer coordinates is formulated in terms of triangle comparison instead in terms of Ricci curvature. 
For the rest of this chapter we need the following domains

$$
\begin{aligned}
& B_{0}:=\mathrm{B}\left[0, e^{-(\Theta+1)} r\right], \\
& B_{1}:=\mathrm{B}\left[0,2 e^{\Theta+1} r\right], \\
& B_{2}:=\mathrm{B}\left[0,4 e^{2(\Theta+1)} r\right], \\
& B_{3}:=\mathrm{B}\left[0,4 e^{3(\Theta+1)} r\right] .
\end{aligned}
$$

Further let

$$
\begin{aligned}
& \vec{e}:=((1,0, \ldots, 0),(0,1,0 \ldots, 0), \ldots,(0, \ldots, 0,1) \\
&(-1,0, \ldots, 0),(0,-1,0, \ldots, 0), \ldots,(0, \ldots, 0,-1)) \in\left(\mathbb{R}^{d}\right)^{2 d}
\end{aligned}
$$

be the canonical unit vectors.

Lemma 3.12. Let $\Theta, \varrho, c, \delta>0$. There is some $\sqrt{3.12}>0$ such that for $r \leq \sqrt{3.12}$ and any chart $\varphi: \mathrm{B}(0, \varrho) \rightarrow M$ to a Riemannian manifold $M$ with $\|\varphi\|_{\mathrm{C}^{\alpha}}^{\varrho} \leq \Theta$

$$
\left|\measuredangle_{\text {Eucl. }} \xi 0 \eta-\tilde{\measuredangle}_{\varphi^{*} g} \xi 0 \eta\right|<\delta
$$

for all $x, y \in \mathrm{B}_{g}[p, c r] \backslash \mathrm{B}_{g}(p, r)$ and $\xi:=\varphi^{-1}(x), \eta:=\varphi^{-1}(y)$.

Proof. Assume that for arbitrarily small $r>0$ there is a Riemannian manifold $M$ with $\|(M, p)\|_{\mathrm{C}^{\alpha}}^{\varrho} \leq \Theta$ such that there is a chart $\varphi:(\mathrm{B}(0, \varrho), p) \rightarrow(M, p)$ with $\left|\measuredangle_{\text {Eucl. }} \xi 0 \eta-\tilde{\measuredangle}_{\varphi^{*} g} \xi 0 \eta\right| \geq \delta$ for some $x, y \in \mathrm{B}[0, c r] \backslash \mathrm{B}(0, r)$ and $\xi:=\varphi^{-1}(x)$, $\eta:=\varphi^{-1}(y)$. Let $r_{n}$ be a sequence of such $r$ converging to 0 and $\left(M_{n}, g_{n}\right), \varphi_{n}$, and $x_{n}, y_{n} \in \mathrm{B}_{g_{n}}\left[0, c r_{n}\right] \backslash \mathrm{B}_{g_{n}}\left(0, r_{n}\right)$ corresponding manifolds, charts and points with $\left\|\varphi_{n}\right\|_{C^{\alpha}}^{\varrho} \leq \Theta$ and $\left|\measuredangle_{\text {Eucl. }} \xi_{n} 0 \eta_{n}-\tilde{\measuredangle}_{\varphi^{*} g} \xi_{n} 0 \eta_{n}\right| \geq \delta$ where $\xi:=\varphi_{n}\left(x_{n}\right)$ and $\eta:=\varphi_{n}(\eta)$.

We rescale the metrics by factors $r_{n}^{-1}$, i.e. $r_{n}^{-2}\left(\varphi^{*} g^{n}\right)$. Further, by a linear transformation $A:\left(\mathbb{R}^{d},\left.g_{n}\right|_{0}\right) \rightarrow\left(\mathbb{R}^{d},\langle., .\rangle_{\text {Eucl. }}\right)$ we can assume that $r_{n}^{-2}\left(\varphi^{*} g^{n}\right)$ is Euclidean at the origin. Due to the bounds $\|\mathrm{T} \varphi\| \leq e^{\Theta},\left\|\mathrm{T} \varphi^{-1}\right\| \leq e^{\Theta}$ and since $r_{n} \stackrel{n \rightarrow \infty}{\longrightarrow} 0$, theses new metric are defined on $\mathrm{B}(0, \varrho)$ for sufficiently large $n$, let's denote them by $\tilde{g}_{n}:=\left(A^{-1}\right)^{*}\left(r_{n}^{-2}\left(\varphi^{*} g^{n}\right)\right)$. Further let $\tilde{\xi}_{n}:=A^{-1} \xi_{n}$ and $\tilde{\eta}_{n}:=A^{-1} \eta_{n}$. They are located in the compact set $\mathrm{B}\left[0, \frac{1}{c} e^{2 \Theta} \varrho\right] \backslash \mathrm{B}\left(0, \frac{1}{c} e^{-2 \Theta} \varrho\right)$ due to 1.20 and $\left\|\mathrm{T} \varphi^{-1}\right\| \leq e^{\Theta}$. Due to Arzelà-Ascoli the $\tilde{g}^{n}$ 's subconverge to some metric $\tilde{g}$. By the Hölder semi-norm bounds $\|\left(\varphi^{*} g_{n}\right)$.. $\|_{\mathrm{C}^{\alpha}} \leq \varrho^{-\alpha} \Theta$ this metric is constant. Hence it is Euclidean because each $\tilde{g}_{n}$ is Euclidean at the origin. Since $\tilde{\xi}_{n}$ and $\tilde{\eta}_{n}$ are in a compact set, we can assume that the sequences $\tilde{\xi}_{n}$ and $\tilde{\eta}_{n}$ converge by going to a subsequence. Let $\tilde{\xi}$ and $\tilde{\eta}$ denote the limit points. Observe that

$$
\tilde{\measuredangle}_{\tilde{g}_{n}} \tilde{\xi}_{n} 0 \tilde{\eta}_{n}=\arccos \frac{\left|\tilde{\xi}_{n} 0\right|^{2}+\left|0 \tilde{\eta}_{n}\right|^{2}-\left|\tilde{\xi}_{n} \tilde{\eta}_{n}\right|^{2}}{2\left|\tilde{\xi}_{n} 0\right|\left|\tilde{\eta}_{n} 0\right|} \stackrel{n \rightarrow \infty}{\longrightarrow} \tilde{\measuredangle}_{\text {Eucl. }} \tilde{\xi} 0 \tilde{\eta}
$$

since $\left|\tilde{\xi}_{n} 0\right|$ and $\left|\tilde{\eta}_{n} 0\right|$ are bounded from below. Hence we have

$$
\delta \leq \liminf _{n \rightarrow \infty}\left|\tilde{\measuredangle}_{\text {Eucl. }} \tilde{\xi}_{n} 0 \tilde{\eta}_{n}-\tilde{\measuredangle}_{\tilde{g_{n}}} \tilde{\xi}_{n} 0 \tilde{\eta}_{n}\right|=\left|\tilde{\measuredangle}_{\text {Eucl. }} \xi 0 \tilde{\eta}-\tilde{\measuredangle}_{\text {Eucl. }} \xi 0 \tilde{\eta}\right|=0 .
$$

This is a contradiction, hence the lemma follows. 
Proposition 3.13 (normalization of strainers). Fix $\alpha \in(0,1)$ and $\varrho, \Theta>0$. Choose $\sqrt{3.12}=\sqrt{3.12}(\Theta, \varrho, c, \delta)>0$ according to Lemma 3.12 for $c=2$. Then there is some angle $\varepsilon>0$ and some $\delta>0$ such that for any $r \in\left[0, \frac{1}{5} e^{-3(\Theta+1)} \wedge\right.$ $e^{-\Theta-1} \varrho \wedge r$ [3.12] [1] there is $\Xi=\Xi(d, \varepsilon, \Theta, r)$ such that the following holds:

For any $(M, g, p) \in \mathrm{P}_{\mathrm{C}^{\alpha} \leq \Theta}^{d, \varrho \text { har }}$, E-strainer $\vec{z} \subset \mathrm{B}_{g}[p, 2 r] \backslash \mathrm{B}_{g}(p, r)$ of $p$, and harmonic chart $\psi: \mathrm{B}(0, \varrho) \rightarrow M$ with $\|\psi\|_{\mathrm{C}^{\alpha}}^{\varrho} \leq \Theta$ there is a chart $\varphi: \mathrm{B}(0, \varrho) \rightarrow$ $M$ centered at $p$ of the form $\varphi=\psi \circ \tilde{\varphi}$ with

$$
\|\varphi\|_{\mathrm{C}^{\alpha}}^{\varrho} \leq \Xi, \quad \varphi(2 r \vec{e})=\vec{z}, \quad \varphi(x)=\psi(x)
$$

for all $x \notin B_{1} \backslash B_{0}$.

Proof. Set $\vec{\zeta}:=\psi^{-1}(\vec{z})$. In this proof, let $|$.$| refer to Euclidean distance. Note$ that $e^{-\Theta} r \leq\left|0 \zeta_{i}\right| \leq 2 e^{\Theta} r$ for $i=1, \ldots, d$ due to 1.20 .

We use $\measuredangle \xi 0 \eta$ to denote the Euclidean angle on $\mathrm{B}(0, \varrho)$, that coincides with the comparison angle with respect to Euclidean metric as defined in (1.4), and $\widetilde{\measuredangle} \xi 0 \eta$ to denote the comparison angle with respect to $\psi^{*} g$. We note that we have the triangle inequality for (non-comparison) angles [BBI01, Theorem 3.6.34]

$$
\measuredangle \xi 0 \zeta \leq \measuredangle \xi 0 \eta+\measuredangle \eta 0 \zeta
$$

for any $\xi, \eta, \zeta \in \mathrm{B}(0, \varrho)$.

First, by rotation we may assume that $\measuredangle \zeta_{1}^{+} 0 e_{1}=0$. In the second step observe that $\left|\measuredangle \zeta_{2}^{+} 0 e_{1}-\frac{\pi}{2}\right| \leq\left|\widetilde{\measuredangle} \zeta_{2}^{+} 0 e_{1}-\frac{\pi}{2}\right|+\delta \leq \varepsilon+\delta$ by Lemma 3.12 and 3.5a. Hence can apply a rotation fixing the $e_{1}$-axis such that $\measuredangle \zeta_{2}^{+} 0 e_{2} \leq \varepsilon+\delta$. In the third step we observe that again

$$
\begin{aligned}
& \left|\measuredangle \zeta_{3}^{+} 0 e_{1}-\frac{\pi}{2}\right|=\left|\measuredangle \zeta_{3}^{+} 0 \zeta_{1}^{+}-\frac{\pi}{2}\right| \leq \varepsilon+\delta \quad \text { and } \\
& \left|\measuredangle \zeta_{3}^{+} 0 e_{2}-\frac{\pi}{2}\right| \stackrel{3.8}{\leq}\left|\measuredangle \zeta_{3}^{+} 0 \zeta_{2}-\frac{\pi}{2}\right|+\varepsilon \\
& \underset{\leq}{\operatorname{Lemma}}\left|\widetilde{\measuredangle} \zeta_{3}^{+} 0 \zeta_{2}^{+}-\frac{\pi}{2}\right|+\delta+\varepsilon \\
& \stackrel{3.5 a}{\leq} \delta+2 \varepsilon \text {. }
\end{aligned}
$$

Therefore due to the triangle inequality for angles the (Euclidean) angle between $\zeta_{3}^{+}$and the orthogonal complement of the $e_{1}-e_{2}$-plane is smaller than $(\varepsilon+\delta)+$ $(\delta+2 \varepsilon)$. Hence, provided $d \geq 4$, we can find a rotation fixing the $e_{1}$ - and $e_{2}$-axes such that $\measuredangle e_{3} 0 \zeta_{3}^{+} \leq 2 \delta+3 \varepsilon$. We can repeat this process until $d-1$-th step. In the $d$-th step the orthogonal complement of the $e_{1}-\ldots-e_{d-1}$-hyperplane is simply a line on which $e_{d}$ is located, thus $\measuredangle e_{d} 0 \zeta_{d}^{+}$is immediately bounded in terms of $\varepsilon$ and $\delta$. As the described process comprises only finitely many steps we can make $\measuredangle \zeta_{i}^{+} 0 e_{i}, i=1, \ldots, d$, as small as we wish by choice of $\varepsilon$ and $\delta$, let's say $\frac{\pi}{16}$. Assuming further that $\varepsilon<\frac{\pi}{16}$, we get $\measuredangle \zeta_{i}^{-} 0 e_{i} \leq \frac{\pi}{8}$ for $i=1, \ldots, d$ by 
(3.5b). The resulting arrangement can be depicted as follows:

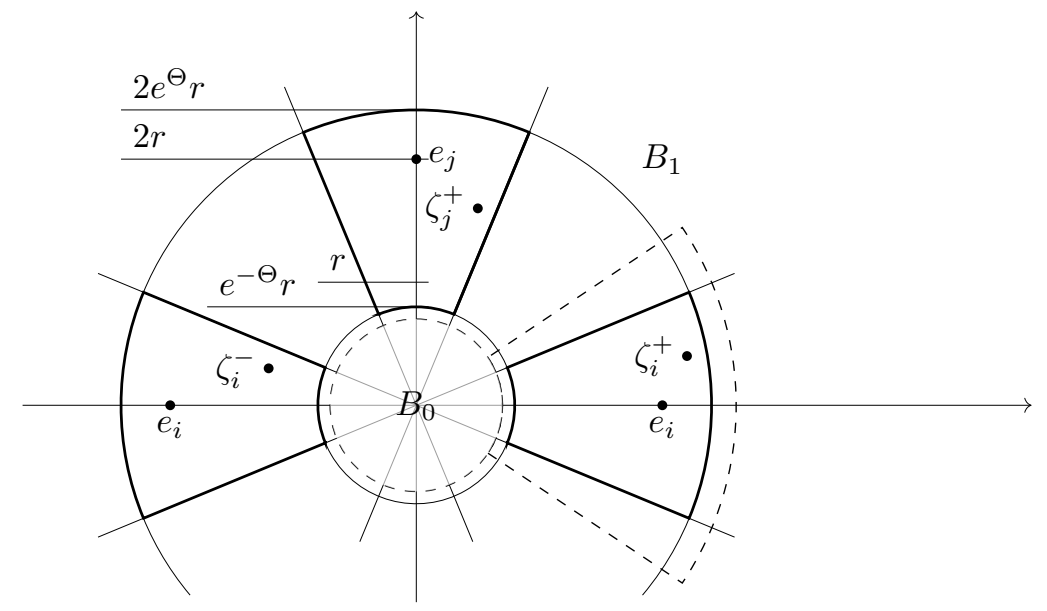

The idea is now to construct an auxiliary function $f^{\prime}$ depending on $r^{-1} \vec{\zeta} \subset \mathbb{R}^{d}$ with the case $r=1$ in mind. We have $r^{-1} \vec{\zeta} \subset \mathrm{B}\left(0,2 e^{\Theta}\right) \backslash \mathrm{B}\left(0, e^{-\Theta}\right)$. Let $K$ be the subset of $\mathrm{B}\left(0,2 e^{\Theta}\right) \backslash \mathrm{B}\left(0, e^{-\Theta}\right)$ consisting of all points that are within a cone of aperture $\frac{3}{8} \pi$ with axis going through one of the $\pm e_{i}$ 's. As $\measuredangle \zeta_{i}^{+} 0 e_{i}, \zeta_{i}^{-} 0\left(-e_{i}\right) \leq \frac{\pi}{8}$ for $i=1, \ldots, d$, we can find some $\mathrm{C}^{1, \alpha}$-isomorphism $f^{\prime}: \mathrm{B}\left(0, r^{-1} \varrho\right) \rightarrow \mathrm{B}\left(0, r^{-1} \varrho\right)$ such that $f^{\prime}: e_{i} \mapsto \zeta_{i}$ for $i=1, \ldots, d$ and $f^{\prime}(\xi)=\xi$ for any $\xi \notin K$-where by $\mathrm{C}^{1, \alpha}$-isomorphism we mean that there is a $\mathrm{C}^{1, \alpha}$-bounded inverse. Both $\mathrm{C}^{1, \alpha}$-bounds can actually be chosen independent of $\vec{\zeta}$ only depending on $d$ and $\varepsilon$.

For general $r>0$, we define

$$
f(\xi):=r f^{\prime}\left(r^{-1} \xi\right) .
$$

Set $\partial_{i}:=\frac{\partial}{\partial \xi_{i}}$. By definition from Section 1.3 we have

$$
\|f\|_{\mathrm{C}^{1, \alpha}}=\|f\|_{\mathrm{C}^{0}}+\sum_{i}\left\|\partial_{i} f\right\|_{\mathrm{C}^{0}}+\sum_{i}\left\|\partial_{i} f\right\|_{\alpha}
$$

Observe that $\partial_{i} f(\xi)=\left(\partial_{i} f^{\prime}\right)\left(r^{-1} \xi\right)$ and $\frac{h\left(r^{-1} \xi\right)-h\left(r^{-1} \eta\right)}{|\xi-\eta|^{\alpha}}=r^{-\alpha} \frac{h\left(r^{-1} \xi\right)-h\left(r^{-1} \eta\right)}{\left|r^{-1} \xi-r^{-1} \eta\right|^{\alpha}}$ for any function $h$, e.g. $h=\partial_{i} f$. Hence

$$
\begin{aligned}
& =r\|f\|_{\mathrm{C}^{0}}+\sum_{i}\left\|\partial_{i} f\right\|_{\mathrm{C}^{0}}+r^{-\alpha} \sum_{i}\left\|\partial_{i} f_{r}\right\|_{\alpha} \\
& \leq r^{-\alpha}\|f\|_{\mathrm{C}^{1, \alpha}} .
\end{aligned}
$$

Analoguously, the inverse is subject to

$$
\left\|f^{-1}\right\|_{\mathrm{C}^{1, \alpha}} \leq r^{-\alpha}\left\|f^{\prime-1}\right\|_{\mathrm{C}^{1, \alpha}} .
$$

Now we have to choose $\Xi$ so large such that it bounds the $\mathrm{C}^{1, \alpha}$-norm of the chart $\varphi:=\psi \circ f$ on the scale of $\varrho$ according to Definition 1.20 To force the first condition choose $\Xi$ so large that for the differentials we have

$$
\begin{aligned}
\|\mathrm{T}(\psi \circ f)\| & =\|\mathrm{T} f \circ \mathrm{T} \psi\| \leq\|\mathrm{T} f\| \cdot\|\mathrm{T} \psi\| \leq e^{\Xi} \text { and likewise } \\
\left\|\mathrm{T}(\psi \circ f)^{-1}\right\| & \leq\left\|\mathrm{T} \psi^{-1}\right\| \cdot\left\|\mathrm{T} f^{-1}\right\| \leq e^{\Xi} .
\end{aligned}
$$


For the second condition observe that the $\|\cdot\|_{\alpha}$-seminorm pullback metric $f^{*} g$ is bounded according to Corollary 1.11 in terms of a constant $C=C(\varrho)$ and $\|f\|_{\mathrm{C}^{\alpha}}$ and $\left\|g_{. .}\right\|_{\mathrm{C}^{\alpha}}$. Observe that $\left\|g_{. .}\right\|_{\mathrm{C}^{\alpha}}$ is bounded in terms of $d, \varrho, \alpha$, and $\Theta$ since $\left\|g_{. .}\right\|_{\mathrm{C}^{0}} \leq d^{2} e^{\Theta}$ due to $\left\|\mathrm{T} \varphi^{-1}\right\| \leq \Theta$ and $\|g . .\|_{\alpha} \leq \varrho^{-\alpha} \Theta$. Moreover $\|f\|_{\mathrm{C}^{\alpha}}$ is bounded by $r^{-\alpha}\left\|f^{\prime-1}\right\|_{C^{1, \alpha}}$ as we observed above. Hence $f^{*} g$ is bounded in terms of $d, \varrho, \alpha, \Theta$, and $r$. This allows us to choose a sufficiently large $\Theta$ such that $\varrho^{\alpha}\left\|f^{*} g\right\|_{\alpha} \leq \Theta$.

\subsubsection{Automorphism rigidity}

For any points $x, y$ in a Riemannnian manifold $M$ and any $v \in \mathrm{T}_{x} M$ denote by $\measuredangle(y, x, v)$ the angle between the minimal geodesic from $x$ to $y$ and $v$ provided that such a geodesic uniquely exists. Moreover let $S$ denote a $(d-1$-dimensional $)$ hyperplane in Euclidean space $M=\mathbb{R}^{d}$ containing $x$. Denote by $\measuredangle(S, x, v)$ the angle between the plane $S$ and the vector $v$. Recall from (3.7) that $\vec{e}=$ $\left( \pm e_{1}, \ldots, \pm e_{d}\right)$ are the Euclidean standard unit vectors.

Definition 3.14 (Anderson-Cheeger limit). Let $\alpha>0, \Lambda \in \mathbb{R}, i>0, \Theta>0$, $\varrho \in\left(0, e^{-\Theta} i / 4\right), r \in\left(0, \frac{1}{2} e^{-2 \Theta} \varrho\right)$, and some $\delta \in(0,1)$. We call a Riemannian metric $g$ on $\mathrm{B}(0, \varrho)$ an Anderson-Cheeger limit of exponent $\alpha$ if there exist a chart $\varphi:(\mathrm{B}(0, \varrho), 0) \rightarrow(M, p)$ to some pointed Riemannian manifold $\left(M, g^{\prime}, p\right)$ such that

- $g=\varphi^{*} g^{\prime}$

- $\|\varphi\|_{\mathrm{C}^{\alpha}}^{\varrho} \leq \Theta$ (where $\|\cdot\|_{\mathrm{C}^{\alpha}}$ is the chart norm from Definition 1.20 , and

- $(M, p)$ is the PGH-limit of some smooth Riemannian manifolds $M_{n}$ with $\operatorname{Ric} M_{n} \geq-\Lambda$ and $\operatorname{inj} M_{n} \geq i$.

Theorem 3.15 (automorphism rigidity of limit metrics). Let $\alpha>0, \Lambda \in \mathbb{R}$, $i>0, \Theta>0$, and $\varrho \in\left(0, e^{-\Theta} i / 4\right)$. For sufficiently small radius $r \in\left(0, \frac{1}{2} e^{-2 \Theta} \varrho\right)$ and some $\delta \in(0,1)$ the following holds:

Assume that $g$ is an Anderson-Cheeger limit on $\mathrm{B}(0, \varrho)$ and a locally $g$-distance preserving map $f: \mathrm{B}\left[0,2 e^{2 \Theta} r\right] \rightarrow \mathrm{B}(0, \varrho)$. If $f(r \vec{e})=r \vec{e}$, then $\left.f\right|_{\mathrm{B}[0, \delta r]}=i d$.

The idea of the proof is to show that the geodesics joining the points $r \vec{e}$, geodesics joining points on these geodesics with the points $r \vec{e}$, etc. are dense in $\mathrm{B}[0, \delta r]$ with respect to the Euclidean metric. As we will see, $f$ is the identity map on such points. Hence the claim follows. From Taylor Tay07, Propositions $3.2,3.3,4.2]$ we have a detailed characterization of the limits we are studying:

Lemma 3.16. Assume that a $\mathrm{C}^{\alpha}$-metric $g$ on $\mathrm{B}(0, \varrho)$ is given by a chart $\varphi:(\mathrm{B}(0, \varrho), g, 0) \rightarrow\left(M, g^{\prime}, p\right)$, i.e. $g=\varphi^{*} g^{\prime}$, for a rough pointed Riemannian manifold $\left(M, g^{\prime}, p\right)$ that is the limit of smooth pointed Riemannian manifolds $M_{n}$ such that for some constants $\Lambda$ and $i>0$

- $\operatorname{Ric} M_{n} \geq-\Lambda$ and

- $\operatorname{inj} M_{n} \geq i$.

Then: 
(i) Given $x, y \in \mathrm{B}(0, \varrho)$ with $|x y|_{g^{\prime}} \leq i / 4$ there is a unique geodesic $\gamma$ from $x$ to $y$.

(ii) Assume that $\gamma$ is contained in the chart $\varphi$. Given a sequence $M_{n}=$ $\left(M_{n}, g^{\prime n}, p\right)$ converging to $M$ via some comparison maps $\iota_{n}$ we have that the geodesics $\gamma^{n}$ from $x$ to $y$ with respect to $g^{\prime n}$ converge to $\gamma$ in $\mathrm{C}^{1, \alpha}$.

(iii) if two geodesics $\gamma, \gamma^{\prime}:[0, T] \rightarrow \mathrm{B}(0, \varrho), T>0$, coincide on an open interval $I \subset[0, T]$, then they are identical.

Lemma 3.17. For any plane $S$ through the origin of $\mathbb{R}^{d}$ the is at least one Cartesian coordinate axes in $\vec{e}$ which has a Euclidean angle of at least

$$
\theta_{d}=\frac{\pi}{2}-\arccos \frac{1}{\sqrt{d}}
$$

to the plane. This angle $\theta_{d}$ is positive and depend only on the dimension.

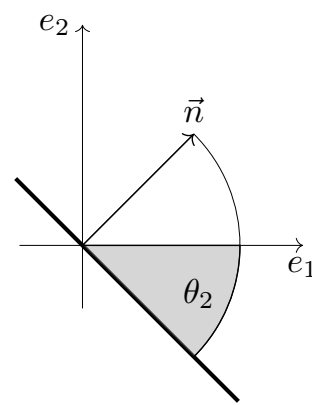

$\theta_{2}=\frac{\pi}{4}$
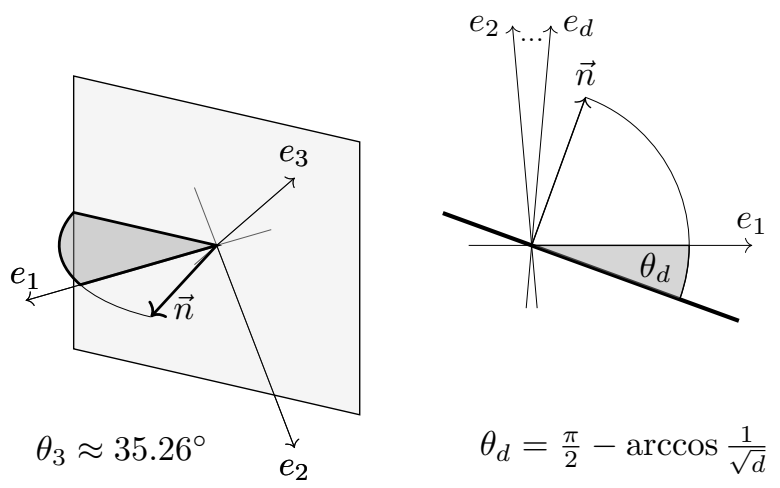

Proof. Given any plane $S$ through the origin let $\vec{n}$ be its normal vector. Instead of minimizing $\max _{e \in \vec{e}} \measuredangle(S, \overrightarrow{0}, e)$ we can as well maximize $f(\vec{n}):=\min _{e \in \vec{e}} \measuredangle(\vec{n}, e)$. Since $\vec{n} \in \mathbb{S}^{d-1} \subset \mathbb{R}^{d}$, the supremum in dependence of $\vec{n}$ is obtained. By symmetry we may assume that $\vec{n}$ is in the first quadrant spanned by $e_{1}, \ldots, e_{d}$. The quantity $f(\vec{n})$ is maximal when $\vec{n}=\frac{1}{\sqrt{d}}(1, \ldots, 1)$. Hence

$$
f\left(\frac{1}{\sqrt{d}}\left(\begin{array}{c}
1 \\
\vdots \\
1
\end{array}\right)\right)=\min _{i=1, \ldots, n} \arccos \left\langle e_{i}, \frac{1}{\sqrt{d}}\left(\begin{array}{c}
1 \\
\vdots \\
1
\end{array}\right)\right\rangle_{\mathrm{Eucl} .}=\arccos \frac{1}{\sqrt{d}}
$$

Hence $\theta_{d}=\frac{\pi}{2}-f\left(\frac{1}{\sqrt{d}}(1, \ldots, 1)\right)=\frac{\pi}{2}-\arccos \frac{1}{\sqrt{d}}$.

Lemma 3.18. For sufficiently small $\bar{q}_{3.18} \in(0,1)$ we have that for and $r>0$ any plane $S$ through a point $x \in \mathrm{B}\left(0, \delta_{3.18}\right)$ there is a point $e \in \vec{e}$ such that the Euclidean angle $\measuredangle(S, x, r e)$ is at least $\frac{3}{4} \theta_{d}$.

Proof. First look at the case $r=1$. The existence of the required $\delta_{3.18}$ is implied by Lemma 3.17 and continuity of angles. Since angles are scale invariant, the claim holds for arbitrary $r$. 
Lemma 3.19. Let $\oint_{3.19} \in(0,1)$. There is some $\eta_{3.19}$ such that for any $r \leq \eta_{3.19}$ the following holds: For any metric $g$ on $\mathrm{B}(0, \varrho)$ as in Theorem 3.15 the geodesic from $-r e_{1}$ to re $_{1}$ intersects the ball $\mathrm{B}\left[0, \overline{q_{3.19}}\right]$.

Proof. Assume that there is no such $\sqrt{3.19}>0$. This is to say that there is a sequence $r_{n}>0$ going to zero such that for some metric $g^{n}$ on $\mathrm{B}(0, \varrho)$ as in Theorem 3.15 the geodesic from $-r_{n} e_{1}$ to $r_{n} e_{1}$ does not intersect the ball $\mathrm{B}\left[0, \delta_{3.19}\right]$. As we have a uniform $\mathrm{C}^{\alpha}$-bound on the metrics we may assume (by going to a subsequence using the Arzelà-Ascoli theorem) that the $g_{n}$ 's $\mathrm{C}^{\beta}$-converge to some metric $g$. We rescale the metrics by factors $r_{n}^{-1}$, i.e. $\tilde{g}^{n}:=r_{n}^{-2} g^{n}$. With respect to each rescaled metric the geodesic from $-e_{1}$ to $e_{2}$ does not intersects the ball $\mathrm{B}\left[0, \delta_{3.19}\right]$. As $r_{n} \stackrel{n \rightarrow \infty}{\longrightarrow} 0$, the spaces $\left(\mathrm{B}(0, \varrho) \tilde{g}^{n}, 0\right)$ PGH-converge to the Euclidean space $\left(\mathbb{R}^{d}, 0\right)$. Moreover the geodesics from $-e_{1}$ to $e_{1}$ with respect to $\tilde{g}^{n}$ converge to the Euclidean geodesic from $-e_{1}$ to $e_{1}$ (Lemma 3.16 (ii) . On the other hand the distance of the limit geodesic to the origin is at least ${ }_{3.19}$. This is a contradiction. Hence the Lemma holds.

Lemma 3.20. Let $\oint_{\overline{3.20}} \in\left(0, \frac{1}{2}\right)$. There is some radius $\sqrt{3.20} \in\left(0, e^{-2 \Theta} \varrho\right)$ such that for every $r \leq \sqrt{3.20}$, every metric $g$ on $\mathrm{B}(0, \varrho)$ as in Theorem 3.15 the following holds: For every points $y \in \mathrm{B}\left[0, \delta_{\overline{3.20}}\right]$ and $x, z \in \mathrm{B}[0, r]$ with $|y x|_{\text {Eucl. }}$. and $|y z|_{\text {Eucl. }}$ at least $\oint_{3.20}$, the difference of the intrinsic angle $\measuredangle_{g}(x, y, z)$ and the Euclidean angle $\measuredangle_{\text {Eucl. }}(x, y, z)$ is not greater than $\frac{1}{4} \theta_{d}$.

Proof. As in the proof of the previous lemma assume that there is a sequence $r_{n}$ that goes to zero and violates the claim, i.e. there is a metric $g^{n}$ on $\mathrm{B}(0, \varrho)$ as in Theorem 3.15 and points $y_{n} \in \mathrm{B}\left[0, \overline{\bar{\beta}_{3.20}} r_{n}\right]$ and $x_{n}, z_{n} \in \mathrm{B}\left[0, r_{n}\right]$ such that $\left|\measuredangle_{g^{n}}\left(x_{n}, y_{n}, z_{n}\right)-\measuredangle_{\text {Eucl. }}\left(x_{n}, y_{n}, z_{n}\right)\right|>\theta_{d} / 4$ for all $n$. Since in our case geodesics $\mathrm{C}^{1, \alpha}$-converge under PGH-convergence (Lemma 3.16) and the angle $\measuredangle_{g^{n}}\left(x_{n}, y_{n}, z_{n}\right)$ depends on the first derivatives of the geodesics from $y$ to $x$ and $z$, we may assume that each metric $g^{n}$ origins from a metric $g^{n \prime}$ on a manifold $\left(M, g^{n \prime}, p\right)$ with $\operatorname{Ric} M_{n} \geq-\Lambda$ and inj $M_{n} \geq i$.

As in the previous we can further assume that the metrics $g^{n} \mathrm{C}^{\beta}$-converge for some metric $g$ on $\mathrm{B}(0, \varrho)$. We rescale by $\tilde{g}^{n}=r_{n}^{-2} g^{n}$. As $r_{n} \stackrel{n \rightarrow \infty}{\longrightarrow} 0$, the spaces $\left(\mathrm{B}(0, \varrho) \tilde{g}^{n}, 0\right) \mathrm{PGH}$-converge to the Euclidean space $\left(\mathbb{R}^{d}, 0\right)$ via some comparison maps $\iota_{n}$ to $\mathbb{R}^{d}$; more precisely, let $\psi$ a linear transformation that maps the tangent space of $g$ at 0 isometrically to Euclidean space; $\iota_{n}$ can be chosen as $\xi \mapsto \psi\left(r_{n}^{-1} \xi\right)$. Hence we can assume that the $\iota_{n}$ 's are isometric embeddings. The sequences $\iota_{n}\left(x_{n}\right), \iota_{n}\left(y_{n}\right)$, and $\iota_{n}\left(z_{n}\right)$, resp., are contained in the compact ball $\mathrm{B}\left[0, e^{-\Theta}\right]$ by 1.20 . Hence by going to a subsequence we may assume that these sequences converge to some $x, y$, and $z$, resp. with $|y x|_{\text {Eucl. }}$ and $|y z|_{\text {Eucl. }}$ at least $\oint_{3.20}$. We identify $x_{n}$ with $\iota\left(x_{n}\right), y_{n}$ with $\iota\left(y_{n}\right)$, and $z_{n}$ with $\iota\left(z_{n}\right)$. Due to Lemma 3.16 the geodesics from $y$ to $x$ and $z$ are $\mathrm{C}^{1, \alpha}$-limits of the geodesics from $y_{n}$ to $x_{n}$ and $z_{n}$. Let $\psi_{n}: \mathrm{B}(0, \varrho) \rightarrow \mathrm{B}(0, \varrho)$ be transformations that map $x_{n}, y_{n}, z_{n}$ to $x, y, z$, resp. such that $\psi_{n} \rightarrow i d$ in $\mathrm{C}^{1}$-norm. Hence

$$
\begin{aligned}
\frac{1}{4} \theta_{d} & \leq \liminf _{n \rightarrow \infty}\left|\measuredangle_{\tilde{g}^{n}}\left(x_{n}, y_{n}, z_{n}\right)-\measuredangle_{\text {Eucl. }}\left(x_{n}, y_{n}, z_{n}\right)\right| \\
& \leq \liminf _{n \rightarrow \infty}\left|\measuredangle_{\psi_{n}^{*} \tilde{g}^{n}}\left(x_{n}, 0, z_{n}\right)-\measuredangle_{\psi_{n}^{*} \tilde{g}^{n}}(x, 0, z)\right| \\
& =\left|\measuredangle_{\text {Eucl. }}(x, y, z)-\measuredangle_{\text {Eucl. }}(x, y, z)\right|=0 .
\end{aligned}
$$

This is a contradiction. 
Proof of Theorem 3.15. Let $\delta=\frac{1}{4} \delta_{3.18} \wedge \frac{1}{4}$ according to Lemma 3.18 Apply Lemma 3.19 to $\delta$ obtaining a radius $\eta_{3.19}$ and apply Lemma 3.20 to $4 \delta=\oint_{3.18}$ obtaining a radius $\eta_{3.20}$. Set $r \leq \eta_{3.19} \wedge r_{3.20} \wedge \frac{1}{2} e^{-2 \Theta} \varrho$.

Due to the injectivity radius assumption and 1.20 each geodesic between two points inside $\varphi(\mathrm{B}[0, r])$ is joined by a unique geodesic inside $\varphi\left(\mathrm{B}\left(0,2 e^{2 \Theta} r\right)\right.$ ). Hence we can define:

$$
\begin{aligned}
A_{0} & :=\bigcup\left\{\gamma(I) \mid \gamma \text { is a geodesic from } e \text { to } e^{\prime} \text { with } e, e^{\prime} \in \vec{e}\right\}, \\
A_{n+1} & :=A_{n} \cup \bigcup\left\{\gamma(I) \mid \begin{array}{r}
\gamma \text { is a geodesic from } e \text { to } x \\
\text { with } e \in \vec{e} \text { and } x \in A_{n}
\end{array}\right\}
\end{aligned}
$$

for $n \geq 1$, and

$$
A_{\infty}:=\bigcup_{n=0}^{\infty} A_{n} .
$$

We claim that $A_{\infty}$ is actually dense in $\mathrm{B}_{\text {Eucl. }}(0, \delta r)$ with respect to the Euclidean metric (and therefore dense in $\mathrm{B}_{\text {Eucl. }}[0, \delta r]$ ). Assume that there is a point $x \in \mathrm{B}_{\text {Eucl. }}(0, \delta r)$ some $\varepsilon>0$ such that

$$
\mathrm{B}_{\text {Eucl. }}(x, \varepsilon) \cap A_{\infty}=\emptyset .
$$

Since $r \leq \sqrt{3.19}$ we have $\varepsilon \leq 2 \delta r$. Assume $\varepsilon$ to be maximal with property 3.10. Hence there is a point $y \in \mathrm{B}_{\text {Eucl. }}[0,3 \delta r]$ such that $|x y|_{\text {Eucl. }}=\varepsilon$.

Let $S$ be the surface tangent to the Euclidean sphere of radius $\varepsilon$ around $x$. Since $3 \delta \leq \delta \overline{3.18}$ we have that for at least one $e \in \vec{e}$ the Euclidean angle $\measuredangle(e, y, S)$ is at least $\frac{3}{4} \delta$. Let $v$ be the projection of $e-y$ on $S-y$. Due to Lemma 3.20 we have that the intrinsic angle $\measuredangle_{g}\left(y+4 \delta \frac{v}{|v|}, y, e\right)$ is at least $\frac{3}{4} \delta-\frac{1}{4} \delta=\frac{1}{2} \delta$. Let $\gamma:\left[0,|y e|_{g}\right] \rightarrow \mathrm{B}[0, \varrho]$ be the $g$-geodesic from $y$ to $e$. Since $\gamma^{\prime}(0)$ points into $\mathrm{B}_{\text {Eucl. }}(x, \varepsilon)$, for some small $t>0$ the point $\gamma(t)$ is contained in $\mathrm{B}_{\text {Eucl. }}(x, \varepsilon)$ but also in $A_{\infty}$. This is a contradiction to 3.10 . Hence points in $A_{\infty}$ are dense in $\mathrm{B}(0, \delta r)$.

Due to uniqueness of geodesics (Lemma 3.16 (i) , every point in $A_{0}$ is preserved by $f$. Hence every point in $A_{1}$ is preserved by $f$, and so forth. Thus $A_{\infty}$ is preserved by $f$. Therefore $\left.f\right|_{\mathrm{B}[0, \delta r]}=i d$.

\subsubsection{Selection lemma}

(Note that in this subsection Lemma 3.9 is used, see remark preceding this thesis) In this subsection we prove a theorem that serves to select a chart at the base point $p$ of a space $M \in \mathrm{P} \underset{\mathcal{M}_{\mathrm{C}^{\alpha}}^{d, \varrho} \leq \Theta}{\text { har }}$ in a way continuous around $M \in \mathrm{P} \underset{\mathcal{M}_{\rightarrow}^{d, \varrho} \leq \Theta \text { har }}{\mathrm{C}^{\alpha} \leq \Theta}$. The proof of the lemma is an application, on one hand, of the theory of elliptic PDEs as presented by Gilbarg and Trudinger GT77, and, on the other hand, of the well-known implicit function theorem for Banach spaces, which is introduced and applied in Section 3.1. In the following theorems we will use the Euclidean balls $B_{0}, B_{1}, B_{2}, B_{3}$ as introduced in (3.6a) to (3.6d).

Theorem 3.21. (Assuming the validity of Lemma 3.9) Let $\alpha, \beta \in(0,1)$ with $\beta<\alpha$. Further let $\alpha>0, \Lambda \in \mathbb{R}, i>0, \Theta>0, \varrho>0$. Finally, let

- $B_{2}^{\prime} \supset B_{2}, B_{3}^{\prime} \supset B_{3}$ be Euclidean balls such that $e^{2(\Theta+1)} B_{2}^{\prime} \subset B_{3}^{\prime}$; 
- $g^{0} \in \mathrm{C}^{\alpha}\left(B_{3}^{\prime}, \mathrm{Sym}^{2}\right)$ be a Riemannian metric that is harmonic on $B_{0}$;

- $2 r \vec{e}$ be an $\varepsilon$-strainer of 0 ;

- $r>0$ be sufficiently small so that Theorem 3.15 holds with respect to $\alpha, \Lambda, i, \Theta$, and $\varrho$;

- $g^{0}$ be an Anderson-Cheeger limit with exponent $\alpha$ (see Definition 3.14);

- $\mathcal{F}=\mathcal{F}_{g_{0}} \subset \mathrm{C}^{1, \alpha}\left(B_{2}^{\prime}, \mathbb{R}^{d}\right)_{2 r \vec{e}}$ be a bounded neighborhood of $i d: x \mapsto x$ such that $f\left(B_{2}^{\prime}\right) \subset B_{3}^{\prime}$ for all $f \in \mathcal{F}$.

Then there exists a neighborhood $\mathcal{G}=\mathcal{G}_{g_{0}} \subset \mathrm{C}^{\beta}\left(B_{3}^{\prime}, \mathrm{Sym}^{2}\right)$ around $g^{0}$ and $a$ continuous map

$$
P: \mathcal{G} \longrightarrow i d+\mathrm{C}^{1, \beta}\left(B_{2}^{\prime}, \mathbb{R}^{d}\right)_{2 r \vec{e}}
$$

from $\mathcal{G}$ to the hyperplane $\left\{i d+f \mid f \in \mathrm{C}^{1, \beta}\left(B_{2}^{\prime}, \mathbb{R}^{d}\right)_{2 r \vec{e}}\right\} \subset \mathrm{C}^{1, \beta}\left(B_{2}^{\prime}, \mathbb{R}^{d}\right)$ such that

(i) $P\left(g^{0}\right)=i d$;

(ii) if $\left.g\right|_{B_{0}}$ is harmonic for some $g \in \mathcal{G}$, so is $\left.\left(P(g)^{*} g\right)\right|_{B_{0}}$;

(iii) for all $g, g^{\prime} \in \mathcal{G}$ with $\left.g\right|_{B_{0}}$ and $\left.g^{\prime}\right|_{B_{0}}$ harmonic:

$$
\left(\exists f \in \mathcal{F}:\left.g^{\prime}\right|_{B_{2}^{\prime}}=f^{*} g\right) \Longrightarrow P(g)^{*} g=P\left(g^{\prime}\right)^{*} g^{\prime} .
$$

The theorem is a consequence of the following lemma. The crucial difference is that the Hölder exponent is not lowered, but there is no freedom to choose the set $\mathcal{F}$ anymore.

Lemma 3.22. (Assuming the validity of Lemma 3.9) Let $\alpha \in(0,1)$. Further let $B$ and $B^{\prime}$ be Euclidean balls in $\mathbb{R}^{d}$ centered at zero such that $B_{0} \subset B \subset B^{\prime}$. Finally, let $g^{0} \in \mathrm{C}^{\alpha}\left(B^{\prime}, \mathrm{Sym}^{2}\right)$ be a Riemannian metric that is harmonic on $B_{0}$, and $2 r \vec{e} \in B \backslash B_{0}$.

Then there are an open neighborhood $\mathcal{F} \subset \mathrm{C}^{1, \alpha}\left(B^{\prime}, \mathbb{R}^{d}\right)$ of $i d$, an open neighborhood $\mathcal{G} \subset \mathrm{C}^{\alpha}\left(B^{\prime}, \mathrm{Sym}^{2}\right)$ of $g^{0}$, and a continuous map

$$
P: \mathcal{G} \rightarrow i d+\mathrm{C}^{1, \alpha}\left(B, \mathbb{R}^{d}\right)_{2 r \vec{e}}
$$

such that

(i) $P\left(g^{0}\right)=i d$;

(ii) if $\left.g\right|_{B_{0}}$ is harmonic for some $g \in \mathcal{G}$, so is $\left.\left(G(g)^{*} g\right)\right|_{B_{0}}$;

(iii) for all $g^{\prime} \in \mathcal{G}$ with $\left.g^{\prime}\right|_{B_{0}}$ harmonic and any metric $g^{\prime \prime}$ on a domain containing $B^{\prime}$ :

$\left(\exists f \in \mathcal{F}: f^{*} g^{\prime \prime}\right.$ is defined and $\left.g^{\prime}=f^{*} g^{\prime \prime}\right) \Longrightarrow P\left(\left.g^{\prime \prime}\right|_{B^{\prime}}\right)^{*} g^{\prime \prime}=P\left(g^{\prime}\right)^{*} g^{\prime}$.

Proof of Theorem 3.21 using Lemma 3.22. First, we claim that for any bounded neighborhood $\mathcal{F}^{\prime} \subset i d+\mathrm{C}^{1, \beta}\left(B_{2}^{\prime}, \mathbb{R}^{d}\right)_{2 r \vec{e}}$ of $i d$ there is a small neighborhood $\mathcal{G}^{\prime \prime} \subset \mathrm{C}^{\beta}\left(B_{3}^{\prime}, \mathrm{Sym}^{2}\right)$ of $g^{0}$ such that for any $g, g^{\prime} \in \mathcal{G}^{\prime \prime}$

$$
\forall h \in \mathcal{F}:\left(h^{*} g=\left.g^{\prime}\right|_{B_{2}^{\prime}} \text { and } h(2 r \vec{e})=2 r \vec{e}\right) \Longrightarrow h \in \mathcal{F}^{\prime} .
$$


To see this assume that there are a $\varepsilon>0$ as well as bounded sequences $h_{n} \in \mathcal{F} \subset \mathrm{C}^{1, \alpha}\left(B_{2}^{\prime}, \mathbb{R}^{d}\right)_{2 r \vec{e}}$ and $g_{n}$ such that $g_{n} \rightarrow g^{0}$ in $\mathrm{C}^{\beta}, h_{n}^{*} g_{n}=\left.g_{n}^{\prime}\right|_{B_{2}^{\prime}}$, $h_{n}(2 r \vec{e})=2 r \vec{e}$, but $\left\|h_{n}-i d_{B_{2}^{\prime}}\right\|_{C^{1, \beta}}>\varepsilon$. Then by Arzelà-Ascoli the sequence $h_{n}$ subconverges in $\mathrm{C}^{1, \beta}$ to some $h$. Of course, the corresponding subsequence of the $g_{n}$ 's subconverges to $g^{0}$. Moreover $h^{*} g^{0}=\left.g^{0}\right|_{B_{2}^{\prime}}$ and $h(2 r \vec{e})=2 r \vec{e}$ but $\left\|h-i d_{B_{2}^{\prime}}\right\|_{\mathrm{C}^{1, \beta}} \geq \varepsilon$. This implies that $h$ is not the identity map in contradiction to our rigidity theorem, Theorem 3.15 applied to $f_{\text {Theorem } 3.15}=h$ and $r_{\text {Theorem } 3.15}=2 r$.

Using this observation we can improve Lemma 3.22 , that we apply to $\alpha=\beta$, $B=B_{2}^{\prime}, B^{\prime}$ a ball between $B_{2}^{\prime}$ and $B_{3}^{\prime}$, and $\left.g^{0}\right|_{B^{\prime}}$, obtaining a neighborhood $\mathcal{F}^{\prime}$ of $i d: B_{2}^{\prime} \rightarrow \mathbb{R}^{d}$, a neighborhood $\mathcal{G}^{\prime}$ of $\left.g^{0}\right|_{B^{\prime}}$, and a map $P^{\prime}: \mathcal{G}^{\prime} \rightarrow \mathcal{F}^{\prime}$. By restricting $\mathcal{F}^{\prime}$ further we can assume that $f\left(B^{\prime}\right) \subset B_{3}^{\prime}$ Now, we apply the last paragraph to $\mathcal{F}^{\prime}$ obtaining some $\mathcal{G}^{\prime \prime}$. Set $\mathcal{G}_{g^{0}}=\mathcal{G}^{\prime} \cap \mathcal{G}^{\prime \prime}$. This set together with $P=P^{\prime} \circ\left(\left.g \mapsto g\right|_{B^{\prime}}\right)$ does the job.

For the proof of Lemma 3.22 and a preliminary lemma let us agree on some convenient notation. For a Euclidean ball $B$ (all balls are again centered at zero) we introduce

$$
\begin{aligned}
\mathcal{F} & :=\mathcal{F}=\mathrm{C}^{1, \alpha}\left(B, \mathbb{R}^{d}\right)_{2 r \vec{e}}, \\
\mathcal{G}_{B} & :=\mathrm{C}^{\alpha}\left(B, \mathrm{Sym}^{2}\right) .
\end{aligned}
$$

Using factorization $3.2 \mathrm{~b}$ we denote by

$$
\mathcal{G}_{\tilde{B} \backslash B}:=\operatorname{ker}\left(\left.g \mapsto g\right|_{B}\right) \subset \mathrm{C}^{\alpha}\left(\tilde{B}, \mathrm{Sym}^{2}\right),
$$

for a ball $\tilde{B} \supset B$, the complement of metrics on $B$ inside the space of metrics on $\tilde{B}$. Finally, denote by

$$
\mathcal{G}_{B}^{\text {har }}:=\left\{g \in \mathcal{G}|g|_{B_{0}} \text { is harmonic }\right\}
$$

where $B_{0} \subset B$ is a Euclidean ball. Note that still $B_{0} \cap\{x \in 2 r \vec{e}\}=\emptyset$.

Additionally, we factorize $\mathcal{G}_{B_{0}}$ using Lemma 3.9 by $\varphi_{\text {har }}:\left(\mathcal{V}^{\text {har }} \oplus \mathcal{E}^{\text {har }}, 0\right) \rightarrow$ $\left(\mathcal{G}_{B_{0}}, g^{0}\right)$ and parameterize the metrics harmonic on $B_{0}$ by

$$
\varphi_{B}^{\text {har }}:=\varphi_{\text {har }} \times\left(\operatorname{id}_{\mathcal{G}_{B \backslash B_{0}}}+\left.g^{0}\right|_{B \backslash B_{0}}\right):\left(\mathcal{G}_{B \backslash B_{0}} \oplus \mathcal{V}^{\text {har }} \oplus \mathcal{E}^{\text {har }}, 0\right) \rightarrow\left(\mathcal{G}_{B}, g^{0}\right)
$$

where $\left.g^{0}\right|_{B \backslash B_{0}}:=\operatorname{pr}_{\mathcal{G}_{B \backslash B_{0}}}\left(g^{0}\right)$. And parameterize the domain of (.)*(.) by

$$
\bar{\varphi}_{B^{\prime}}^{\text {har }}:=\varphi_{B^{\prime}}^{\text {har }} \times \operatorname{id}_{\mathcal{F}}
$$

where $i d: B \rightarrow B^{\prime}$.

Choose a factorization of the differential $D_{0}(.)^{*}($.$) of the pullback map onto$ $B$ around $\left(g^{0}, i d\right)$ according to Lemma 3.8 i.e.

$$
\left(\begin{array}{llll}
\operatorname{pr}_{G_{B 1}} & 0 & S & 0 \\
\operatorname{pr}_{G_{B 2}} & 0 & 0 & 0
\end{array}\right): \mathcal{G}_{B} \oplus \mathcal{G}_{B^{\prime} \backslash B} \oplus \mathcal{F}_{1} \oplus \mathcal{F}_{2} \rightarrow \mathcal{G}_{B 1} \oplus \mathcal{G}_{B 2}
$$

Set

$A:=\left(\varphi_{B}^{\text {har }}\right)^{-1} \circ\left((.)^{*}().\right) \circ \bar{\varphi}_{B^{\prime}}^{\text {har }}: \mathcal{G}_{B} \oplus \mathcal{G}_{B^{\prime} \backslash B} \oplus \mathcal{F}_{1} \oplus \mathcal{F}_{2} \rightarrow \mathcal{G}_{B \backslash B_{0}} \oplus \mathcal{V}^{\text {har }} \oplus \mathcal{E}^{\text {har }}$. 
Lemma 3.23. (Assuming the validity of Lemma 3.9) In the notation above we have that

$$
\mathcal{E}^{\text {har }} \subset D_{0} A\left(\mathcal{G}_{B^{\prime} \backslash B_{0}} \oplus \mathcal{V}^{\text {har }} \oplus \mathcal{F}\right) .
$$

Proof. Assume that there is a nontrivial vector $e_{2} \in \mathcal{E}^{\text {har }}$ that is not in

$$
D_{0} A\left(\mathcal{G}_{B^{\prime} \backslash B_{0}} \oplus \mathcal{V}^{\text {har }} \oplus \mathcal{F}\right) .
$$

This space is generated by the closed subspaces with closed complement $\mathcal{E}_{1}:=$ $D_{0} A(\mathcal{F})=\left(\varphi_{B}^{\text {har }}\right)^{-1}(S(\mathcal{F}))$ and $D_{0} A\left(\mathcal{G}_{B^{\prime} \backslash B_{0}} \oplus \mathcal{V}^{\text {har }}\right)=\mathcal{G}_{B \backslash B_{0}} \oplus \mathcal{V}^{\text {har }}$. Hence it is closed and has a closed complement. Let $\mathcal{E}_{2}$ denote the projection of this complement on $\mathcal{E}^{\text {har }}$. We have $e_{2} \in \mathcal{E}_{2} \backslash\{0\}$. Moreover note that $\mathcal{G}_{B \backslash B_{0}} \oplus \mathcal{V}^{\text {har }}=$ $A\left(\mathcal{G}_{B^{\prime} \backslash B_{0}} \oplus \mathcal{V}^{\text {har }} \oplus 0\right)$. Hence

$$
\mathcal{G}_{B \backslash B_{0}} \oplus \mathcal{V}^{\text {har }}=D_{0} A\left(\mathcal{G}_{B^{\prime} \backslash B_{0}} \oplus \mathcal{V}^{\text {har }}\right) \subset D_{0} A\left(\mathcal{G}_{B^{\prime} \backslash B_{0}} \oplus \mathcal{V}^{\text {har }} \oplus \mathcal{F}\right) .
$$

For each $\lambda>0$ let $e_{1}(\lambda) \in \mathcal{F}$ denote a vector such that

$$
A\left(e_{1}(\lambda)+\lambda e_{2}\right)=0
$$

if such a vector exists. Note that always

$$
A\left(\lambda e_{2}\right)=\left(\varphi_{B}^{\text {har }}\right)^{-1} i d^{*} \varphi_{B^{\prime}}^{\text {har }}\left(\lambda e_{2}\right)=\lambda e_{2} .
$$

If we assume that $e_{1}(\lambda) \in \mathcal{E}_{1}$ exists for sufficiently small $\lambda$ and

$$
e_{1}(\lambda) \stackrel{\lambda \rightarrow 0}{\longrightarrow} 0
$$

then we can argue as follows: Since $A$ is Fréchet differentiable at 0 we have

$$
\begin{aligned}
\lambda e_{2} & =\operatorname{pr}_{\mathcal{E}_{2}}\left(\lambda e_{2}-0\right) \\
& =\operatorname{pr}_{\mathcal{E}_{2}}\left(A\left(\lambda e_{2}\right)-A\left(e_{1}(\lambda)+\lambda e_{2}\right)\right) \\
& =\operatorname{pr}_{\mathcal{E}_{2}}\left(D_{0} A\left(\lambda e_{2}\right)-D_{0} A\left(e_{1}(\lambda)+\lambda e_{2}\right)+r\right) \\
& =\operatorname{pr}_{\mathcal{E}_{2}}\left(-D_{0} A\left(e_{1}(\lambda)\right)+r\right) \\
& =\operatorname{pr}_{\mathcal{E}_{2}} r
\end{aligned}
$$

for some function $r$ : $\operatorname{dom} A \rightarrow \mathcal{G}_{B}$ with $|r(x)| /|x| \rightarrow 0$ as $|x| \rightarrow 0$. But this is a contradiction as $e_{2} \neq 0$.

This is to say that we are left to choose $e_{1}(\lambda)$ in such a way that the continuity statement 3.13a) holds. Applying $\bar{\varphi}_{B^{\prime}}^{\text {har }}$, we see that the defining property of $e_{1}(\lambda)$ is that $\left(i d+e_{1}(\lambda)\right)^{*}\left(\varphi_{B^{\prime}}^{\text {har }}\left(\lambda e_{2}\right)\right)$ is harmonic on $B_{0}$. In other words we are dealing with the boundary value problem

$$
\left\{\begin{aligned}
\Delta_{\lambda}^{\mathrm{w}} u_{k} & =0 \\
\left.u\right|_{\partial B_{0}}\left(x_{k}\right) & =x_{k}
\end{aligned}\right.
$$

for $k=1, \ldots, d$, where $i d+e_{1}(\lambda)=u^{-1}$ and $\Delta_{\lambda}^{\mathrm{w}}$ is the weak Laplace-Beltrami operator corresponding to the metric $g^{\lambda}=\left(g_{i j}^{\lambda}\right)_{i, j=1}^{n}:=\varphi_{B^{\prime}}^{\text {har }}\left(\lambda e_{2}\right)$ from 1.22).

Gilbarg and Trudinger [GT77, pp. 181-183] construct a bounded linear solution operator for a second order elliptic differential operator as under consideration here given by

$$
\text { PI: }\left(\mathrm{W}_{0}^{1,2}\left(B_{0}\right)\right)^{*} \rightarrow \mathrm{W}_{0}^{1,2}\left(B_{0}\right),
$$


where $\mathrm{W}_{0}^{1,2}\left(B_{0}\right)$ denotes the $\mathrm{W}^{1,2}$-closure of $\mathrm{C}_{0}^{1}, \mathrm{C}_{0}^{1}$ the compactly supported functions in $\mathrm{C}^{1}$, and $\left(\mathrm{W}_{0}^{1,2}\right)^{*}$ the dual with the operator norm topology. Let $\mathrm{PI}_{\lambda}$ be the solution operator for $\Delta_{\lambda}^{\mathrm{w}}$. As apparent from the definition of $\mathrm{W}_{0}^{1,2}\left(B_{0}\right)$ this solution operator gives a solution only to the Cauchy boundary problem, i.e. when seeking a function vanishing on the boundary. But as pointed out in the same reference this directly generalizes to the Dirichlet problem $3.13 \mathrm{~b}$ by

$$
\begin{array}{rlrl} 
& \Delta_{\lambda}^{\mathrm{w}}\left(u_{k}-x_{k}\right) & =0 \\
& \sum_{i j} \sqrt{\left|g^{\lambda}\right|} g_{\lambda}^{i j} \partial_{j}\left(u_{k}-x_{k}\right) \partial_{i} & =0 \\
\sum_{i j} \sqrt{\left|g^{\lambda}\right|} g_{\lambda}^{i j} \partial_{j} u_{k} \partial_{i} & =\tilde{x}_{k}^{\lambda}
\end{array}
$$

where $\left|g_{\lambda}\right|=\left|\operatorname{det} g_{i j}^{\lambda}\right|$ and $\tilde{x}_{k}^{\lambda}:=\sum_{i j} \sqrt{\left|g_{\lambda}\right|} g_{\lambda}^{i j} \delta_{k j} \partial_{i}$. Hence our solution is

$$
i d+e_{2}(\lambda):=\left(\mathrm{PI}_{\lambda}\left(i d+\tilde{x}_{k}^{\lambda}\right)_{k=1}^{d}\right)^{-1}
$$

which is well defined since the solution by each operator $\mathrm{PI}_{\lambda}$ is unique. It remains to check that the solution is actually in $\mathrm{C}^{1, \alpha}\left(B_{0}\right)$ and $3.13 \mathrm{a}$.

First, due to the additional boundary regularity - that the solution $x_{k}+$ $\mathrm{PI}_{\lambda}\left(\tilde{x}_{k}^{\lambda}\right)$ of $3.13 \mathrm{~b}$ is in $\mathrm{C}^{1, \alpha}$ —we know by $\mathrm{GT} 77$, Theorem 8.34] that $e_{2}(\lambda)$ is actually a $\mathrm{C}^{1, \alpha}$-function.

Second, we check that actually $\left(\mathrm{PI}_{\lambda}\left(\tilde{x}_{k}^{\lambda}\right)\right)_{k=1}^{d} \stackrel{\lambda \rightarrow 0}{\longrightarrow} 0$ with respect to the $\mathrm{C}^{0}$-norm. Let $\Delta_{0}^{\mathrm{w}}$ and $\mathrm{PI}_{0}$ be the Laplace-Beltrami and solution operators corresponding to $g^{0}$ and $\tilde{x}_{k}^{0}:=\sum_{i j} \sqrt{\left|g^{0}\right|}\left(g^{0}\right)^{i j} \delta_{k j} \partial_{i}$. By assumption $i d+0$ is actually a solution of the problem $3.13 \mathrm{~b}$ for $\lambda=0$, so $\mathrm{PI}_{0}\left(\tilde{x}_{k}^{0}\right)=0$. Thus $\tilde{x}_{k}^{0}=0$. Hence

$$
\left\|\mathrm{PI}_{\lambda}\left(\tilde{x}_{k}^{\lambda}\right)\right\|_{L^{\infty}}=\left\|\mathrm{PI}_{\lambda}\left(\tilde{x}_{k}^{\lambda}-\tilde{x}_{k}^{0}\right)\right\|_{L^{\infty}}
$$

Using an estimate GT77, Theorem 8.16] in the coefficients of the distribution $\tilde{x}_{k}^{\lambda}-\tilde{x}_{k}^{0}=\sum_{i j}\left(\sqrt{\left|g^{\lambda}\right| g_{\lambda}^{i j}}-\sqrt{\left|g^{0}\right|}\left(g^{0}\right)^{i j}\right) \delta_{k j} \partial_{i}$ we obtain

$$
\leq C\left\|\left(\sum_{j}\left(\sqrt{\left|g^{\lambda}\right|} g_{\lambda}^{i j}-\sqrt{\left|g^{0}\right|}\left(g^{0}\right)^{i j}\right) \delta_{k j}\right)_{i=1}^{n}\right\|_{L^{q / 2}}
$$

for $\lambda$ sufficiently small, $q>n$, and $C=C\left(g^{0}, d, q, B_{0}\right)$. But the coefficients $\sum_{j}\left(\sqrt{\left|g_{\lambda}\right|} g_{\lambda}^{i j}-\sqrt{\left|g^{0}\right|}\left(g^{0}\right)^{i j}\right) \delta_{k j}$ converge to 0 in $L^{q / 2}$-norm as $g_{\lambda}$ converges to $g^{0}$ (or actually even in $\mathrm{C}^{\alpha}$ ). Hence $\left(\mathrm{PI}_{\lambda}\left(\tilde{x}_{k}^{\lambda}\right)\right)_{k=1}^{d} \rightarrow 0$ with respect to the $\mathrm{C}^{0}$-norm.

Third, we improve the convergence $\mathrm{PI}_{\lambda}\left(\tilde{x}_{k}^{\lambda}\right) \stackrel{\lambda \rightarrow 0}{\longrightarrow} 0$ in $\mathrm{C}^{0}$ to convergence in $\mathrm{C}^{1, \alpha}$. For the harmonic $\mathrm{C}^{1, \alpha}$-solution $\mathrm{PI}_{\lambda}\left(\tilde{x}_{k}^{\lambda}\right)$ of the Cauchy problem $3.13 \mathrm{c}$ an established Hölder estimate GT77, Theorem 8.33] gives

$$
\left\|\mathrm{PI}_{\lambda}\left(\tilde{x}_{k}^{\lambda}\right)\right\|_{\mathrm{C}^{1, \alpha}} \leq C\left\|\mathrm{PI}_{\lambda}\left(\tilde{x}_{k}^{\lambda}\right)\right\|_{\mathrm{C}^{0}}
$$

where $C$ depends only on bounds to the metric $g^{\lambda}$. Thus the $\mathrm{C}^{0}$-convergence proved in last paragraph is already sufficient to imply $\mathrm{C}^{1, \alpha}$-convergence. Due to Lemma 3.6 the inverses of the $i d+\mathrm{PI}_{\lambda}\left(\tilde{x}_{k}^{\lambda}\right)$ 's $\mathrm{C}^{1, \alpha}$-converge as well. Hence we have 3.13a follows. This completes the proof.

Proof of Lemma 3.22. The proof proceeds is four steps. 
Linearization of functions harmonic on $B_{0}$. Identify $\mathcal{V}^{\text {har }}$ with $\mathcal{G}_{B_{0}}^{\text {har }}$. Further the factorization of $\mathcal{G}_{B^{\prime}}$ and $\mathcal{F}$ according to 3.12 is preserved by $\bar{\varphi}_{B^{\prime}}^{\text {har }}$ in the following sense: Set $T:=D_{0} \bar{\varphi}_{B^{\prime}}^{\text {har }}$ and define

$$
\begin{aligned}
\mathcal{G}_{B_{0} 1}^{\text {har }} & :=\operatorname{pr}_{\mathcal{G}_{B_{0}}^{\text {har }}} \circ \operatorname{pr}_{B_{0}} \circ T^{-1}\left(\mathcal{G}_{B 1}\right) & \mathcal{G}_{B_{0} 2}^{\text {har }} & :=\operatorname{pr}_{\mathcal{G}_{B_{0}}^{\text {har }}} \circ \operatorname{pr}_{B_{0}} \circ T^{-1}\left(\mathcal{G}_{B 2}\right) \\
\mathcal{E}_{B_{0} 1}^{\text {har }} & :=\operatorname{pr}_{\mathcal{E}_{B_{0}}^{\text {har }} \circ \operatorname{pr}_{B_{0}} \circ T^{-1}\left(\mathcal{G}_{B 1}\right)} \mathcal{E}_{B_{0} 2}^{\text {har }}:=\operatorname{pr}_{\mathcal{E}_{B_{0}}^{\text {har }} \circ \operatorname{pr}_{B_{0}} \circ T^{-1}\left(\mathcal{G}_{B 2}\right)} \circ & \mathcal{G}_{B \backslash B_{0} 2} & :=\operatorname{pr}_{\mathcal{G}_{B \backslash B_{0}}} \circ T^{-1}\left(\mathcal{G}_{B 2}\right) .
\end{aligned}
$$

Further define

$$
\mathcal{G}_{11}^{\text {har }}:=\mathcal{G}_{B_{0} 1}^{\text {har }} \oplus \mathcal{G}_{B \backslash B_{0} 1} \quad \mathcal{G}_{12}^{\text {har }}:=\mathcal{G}_{B_{0} 2}^{\text {har }} \oplus \mathcal{G}_{B \backslash B_{0} 2}
$$

With this reparametrization of the domain the map $\tilde{A}^{\text {har }}:=\left((.)^{*}().\right) \circ \bar{\varphi}_{\text {har }}$ has the differential

$$
\begin{aligned}
& D_{0} \tilde{A}^{\text {har }}=\left(\begin{array}{ccccccc}
\tilde{A}_{1} & 0 & 0 & 0 & 0 & \tilde{S}_{1} & 0 \\
0 & \tilde{A}_{2} & 0 & 0 & 0 & 0 & 0 \\
0 & 0 & \tilde{A}_{3} & 0 & 0 & \tilde{S}_{2} & 0 \\
0 & 0 & 0 & \tilde{A}_{4} & 0 & 0 & 0
\end{array}\right): \\
& \mathcal{G}_{11}^{\text {har }} \oplus \mathcal{G}_{12}^{\text {har }} \oplus \mathcal{E}_{B_{0} 1}^{\text {har }} \oplus \mathcal{E}_{B_{0} 2}^{\text {har }} \oplus \mathcal{G}_{B^{\prime} \backslash B} \oplus \mathcal{F}_{1} \oplus \mathcal{F}_{2} \rightarrow \tilde{\mathcal{H}}_{11}^{\text {har }} \oplus \tilde{\mathcal{H}}_{12}^{\text {har }} \oplus \tilde{\mathcal{H}}_{2}^{\text {har }} \oplus \tilde{\mathcal{H}}_{2}
\end{aligned}
$$

where $\tilde{\mathcal{H}}_{11}^{\text {har }}, \tilde{\mathcal{H}}_{12}^{\text {har }}, \tilde{\mathcal{H}}_{2}^{\text {har }}, \tilde{\mathcal{H}}_{2}$ are the suitable closed subspaces and $\tilde{A}_{1}, \tilde{A}_{2}, \tilde{A}_{3}$, $\tilde{A}_{4}$ are linear homeomorphisms and $\tilde{S}_{1}, \tilde{S}_{2}$ are surjective bounded linear maps. Moreover $\left(\tilde{S}_{1}, \tilde{S}_{2}\right): \mathcal{F}_{1} \rightarrow \tilde{H}_{11} \oplus \tilde{H}_{2}^{\text {har }}$ is injective. Note that $\mathcal{G}_{B 1}=\tilde{\mathcal{H}}_{11}^{\text {har }} \oplus \tilde{\mathcal{H}}_{2}^{\text {har }}$ and $\mathcal{G}_{B 2}=\tilde{\mathcal{H}}_{12}^{\text {har }} \oplus \tilde{\mathcal{H}}_{2}$.

We can do for $\mathcal{G}_{B}$ the same decomposition as for $\mathcal{G}_{B^{\prime}}$ by the map $\varphi_{B}^{\text {har }}$, i.e. we can view $\mathcal{G}_{B}^{\text {har }}$ as a linear subspace of $\mathcal{G}_{B}$. As $\tilde{A}\left(\mathcal{G}_{11}^{\text {har }} \oplus \mathcal{G}_{12}^{\text {har }}\right)$ is $\mathcal{G}_{B}^{\text {har }}$, we have that $D_{\left.g^{0}\right|_{B}} \varphi_{B}^{\text {har }}$ is an isomorphism from $\tilde{\mathcal{H}}_{11}^{\text {har }} \oplus \tilde{\mathcal{H}}_{12}^{\text {har }}$ to $\mathcal{V} \oplus \mathcal{G}_{B \backslash B_{0}}$. Define

$$
\begin{array}{ll}
\mathcal{H}_{1}:=D_{g^{0}} \varphi_{B}^{\text {har }}\left(\tilde{\mathcal{H}}_{11}^{\text {har }}\right) & \mathcal{H}_{2}:=D_{g^{0}} \varphi_{B}^{\text {har }}\left(\tilde{\mathcal{H}}_{12}^{\text {har }}\right) \\
\mathcal{H}_{3}:=D_{g^{0}} \varphi_{B}^{\text {har }}\left(\tilde{\mathcal{H}}_{2}^{\text {har }}\right) & \mathcal{H}_{4}:=D_{g^{0}} \varphi_{B}^{\text {har }}\left(\tilde{\mathcal{H}}_{2}\right) .
\end{array}
$$

Lemma 3.23 amounts to the fact that $\mathcal{H}_{3} \oplus \mathcal{H}_{2} \subset D_{0} A\left(\mathcal{G}_{11}^{\text {har }} \oplus \mathcal{G}_{12}^{\text {har }} \oplus \mathcal{G}_{B^{\prime} \backslash B} \oplus\right.$ $\left.\mathcal{F}_{1} \oplus \mathcal{F}_{2}\right)$. But this is to say that $\mathcal{H}_{4}=0$. Hence $\mathcal{E}_{B_{0} 2}^{\text {har }}=0$ as well. Moreover Lemma 3.23 implies that $\mathcal{E}^{\text {har }} \subset\left(\tilde{S}_{1}, \tilde{S}_{2}\right)\left(\mathcal{F}_{1}\right)$. To sum this up, the fourth row in the matrix representation of $D_{0} \tilde{A}^{\text {har }}$ cancels and $\left(\tilde{S}_{1}, \tilde{S}_{2}\right)$ is a linear homeomorphism onto $\tilde{H}_{11} \oplus \tilde{H}_{2}^{\text {har }}$. For convenience define

$$
\begin{aligned}
& \mathcal{G}_{1}:=\mathcal{G}_{11}^{\text {har }}, \quad \mathcal{G}_{2}:=\mathcal{G}_{12}^{\text {har }}, \quad \mathcal{G}_{3}:=\mathcal{E}_{B_{0} 1}^{\text {har }}, \\
\mathcal{G}:= & \mathcal{G}_{1} \oplus \mathcal{G}_{2} \oplus \mathcal{G}_{3} \oplus \mathcal{G}_{B^{\prime} \backslash B}, \quad \mathcal{H}:=\mathcal{H}_{1} \oplus \mathcal{H}_{2} \oplus \mathcal{H}_{3} .
\end{aligned}
$$

In this reparametrization the differential of $A$ becomes

$$
\begin{aligned}
D_{0} A=\left(\begin{array}{ccccccc}
A_{1} & 0 & 0 & 0 & S_{1} & 0 & 0 \\
0 & A_{2} & 0 & 0 & 0 & 0 & 0 \\
0 & 0 & A_{3} & 0 & 0 & S_{2} & 0
\end{array}\right): \\
\mathcal{G}_{1} \oplus \mathcal{G}_{2} \oplus \mathcal{G}_{3} \oplus \mathcal{G}_{B^{\prime} \backslash B} \oplus \mathcal{F}_{1} \oplus \mathcal{F}_{2} \rightarrow \mathcal{H}_{1} \oplus \mathcal{H}_{2} \oplus \mathcal{H}_{3}
\end{aligned}
$$

where $A_{1}, A_{2}, A_{3}$ and $S_{1}, S_{2}$ are linear homeomorphisms. 
Quotient out $\mathcal{F}_{2}$ by $\varphi$. Consider the map

$$
\tilde{B}:=\left(A, \operatorname{pr}_{\mathcal{G}_{1} \oplus \mathcal{G}_{3} \oplus \mathcal{G}_{B^{\prime} \backslash B}}\right): \mathcal{G} \oplus \mathcal{F}_{1} \oplus \mathcal{F}_{2} \rightarrow \mathcal{H} \oplus\left(\mathcal{G}_{1} \oplus \mathcal{G}_{3} \oplus \mathcal{G}_{B^{\prime} \backslash B}\right) .
$$

The kernel of the differential $D_{0} B$ is given by $\mathcal{F}_{2}$. Choose the obvious complement $\mathcal{G} \oplus \mathcal{F}_{1}$. Applying Lemma 3.4 provides a locally defined map

$$
\varphi:\left(\mathcal{G} \oplus \mathcal{F}_{1}\right) \oplus \mathcal{F}_{2} \rightarrow\left(\mathcal{G} \oplus \mathcal{F}_{1}\right) \oplus \mathcal{F}_{2}
$$

such that, by (3.3a), $A \circ \varphi\left(e_{1}, e_{2}\right)$ is constant in $e_{2} \in \mathcal{F}_{2}$ and, by (3.3b), $\varphi\left(e_{1}, e_{2}\right)=$ $e_{1}+\varphi_{2}\left(e_{1}, e_{2}\right)$ with $\varphi_{2}\left(e_{1}, e_{2}\right) \in \mathcal{F}_{2}$. Setting $B:=\left.A \circ \varphi\right|_{\mathcal{G} \oplus \mathcal{F}_{1} \oplus 0}$ we get that

$$
D_{0} B=\left(\begin{array}{ccccc}
A_{1} & 0 & 0 & 0 & S_{1} \\
0 & A_{2} & 0 & 0 & 0 \\
0 & 0 & A_{3} & 0 & S_{2}
\end{array}\right): \mathcal{G} \oplus \mathcal{F}_{1} \rightarrow \mathcal{H}
$$

Construct $P$ by implicit function theorem. Consider the map

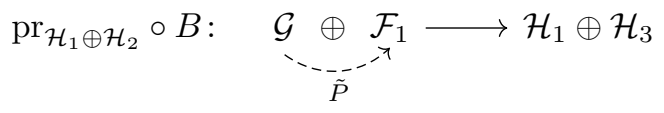

At 0 the restricted differential $\left.D_{0} \operatorname{pr}_{\mathcal{H}_{1} \oplus \mathcal{H}_{2}} \circ B\right|_{\mathcal{F}_{1}}$ is $S_{1} \oplus S_{2}$ and hence invertible. Moreover $\operatorname{pr}_{\mathcal{H}_{1} \oplus \mathcal{H}_{2}} \circ B(0,0)=0$. Hence - after restricting the domain of $B$ accordingly - the standard implicit function theorem for Banach spaces Die69. $\S 10.2$ ] provides a locally defined map $\tilde{P}: \mathcal{G} \rightarrow \mathcal{F}_{1}$ with domain of definition $\mathcal{G}^{\prime}$ such that

$$
\operatorname{pr}_{\mathcal{H}_{1} \oplus \mathcal{H}_{2}} \circ B(g, \tilde{P}(g))=0
$$

uniquely for $g \in \mathcal{G}^{\prime}$ and $\tilde{P}(g) \in \mathcal{F}^{\prime}$ for some $\mathcal{F}^{\prime} \subset \mathcal{F}_{1}$. Now for $g$ in a sufficiently small neighborhood of $g^{0}$ (i.e. such that $\bar{\varphi}_{\text {har }}(g, i d)$ is in the domain of $B$ ) define

$$
\begin{aligned}
\widetilde{g} & :=\operatorname{pr}_{\mathcal{G}} \circ \varphi_{\text {har }}^{-1}(g, i d) \\
P(g) & :=\operatorname{pr}_{\mathcal{G}} \circ \varphi(\widetilde{g}, \tilde{P}(\widetilde{g})) .
\end{aligned}
$$

Note that $\widetilde{g}=\operatorname{pr}_{\mathcal{G}} \circ \varphi^{-1} \circ \varphi_{\text {har }}^{-1}(g, i d)$ and that $(g, P(g))=\varphi(\widetilde{g}, \tilde{P}(\widetilde{g})$. Further let $\mathcal{F}^{\prime \prime}$ be a neighborhood of 0 in $\mathcal{F}_{2}$ such that $\mathcal{G}^{\prime} \times \mathcal{F}^{\prime} \times \mathcal{F}^{\prime \prime}$ is in the domain of $\varphi$ (if necessary, restrict $\mathcal{G}^{\prime}$ and $\mathcal{F}^{\prime}$ further). Let $\tilde{\mathcal{G}} \times \tilde{\mathcal{F}}$ be a neighborhood of $\left(g^{0}, i d\right)$ such that $\tilde{\mathcal{G}} \times \tilde{\mathcal{F}} \subset \bar{\varphi}_{B^{\prime}}^{\text {har }} \circ \varphi\left(\mathcal{G}^{\prime} \times \mathcal{F}^{\prime} \times \mathcal{F}^{\prime \prime}\right)$. By Corollary 1.14 , the continuity of $P$, and $P\left(g^{0}\right)=i d$, it is possible to find neighborhoods $\mathcal{\mathcal { G }} \subset \tilde{\mathcal{G}}$ of $g^{0}$ and $\mathcal{F}$ of $i d \in \mathrm{C}^{1, \alpha}\left(B^{\prime}, \mathbb{R}^{d}\right)$ such that $f^{\prime} \circ P(g) \in \tilde{\mathcal{F}}$ for all $g \in \mathcal{G}$ and $f^{\prime} \in \mathcal{F}$.

Check that $P(g)$ does the job. First observe $\widetilde{g^{0}}=0$. Hence $\tilde{P}\left(\widetilde{g^{0}}\right)=0$ and, hence, $P\left(g^{0}\right)=i d$. This is claim (i) Further observe that $P(g)^{*} g=B(\widetilde{g}, P(\widetilde{g}))$ and, hence, the $\mathcal{H}_{3}$ component vanishes, i.e. $P(g)^{*} g$ is harmonic on $B_{0}$. This is claim (ii)

To check the uniqueness property claim (iii) assume that there is some $g^{\prime}$ in $\mathcal{G}$ harmonic on $B_{0}$ and some metric $g^{\prime \prime}$ on a larger domain such that for some $f \in \mathcal{F}$ the pullback $f^{*} g^{\prime \prime}$ is defined and $g^{\prime}=f^{*} g^{\prime \prime}$. It follows that $P\left(g^{\prime}\right)^{*} g^{\prime}=P\left(g^{\prime}\right)^{*} f^{*} g^{\prime \prime}=\left(f \circ P\left(g^{\prime}\right)\right)^{*} g^{\prime \prime}$. Moreover $\left(f \circ P\left(g^{\prime}\right)\right)^{*}\left(\left.g^{\prime \prime}\right|_{B^{\prime}}\right)$ is defined as $f \circ P\left(g^{\prime}\right) \in \tilde{\mathcal{F}}$. By the equality $P\left(g^{\prime}\right)^{*} g^{\prime}=\left(f \circ P\left(g^{\prime}\right)\right)^{*}\left(\left.g^{\prime \prime}\right|_{B^{\prime}}\right)$ we have 
$\operatorname{pr}_{\mathcal{H}_{1} \oplus \mathcal{H}_{2}}\left(f \circ P\left(g^{\prime}\right)\right)^{*}\left(\left.g^{\prime \prime}\right|_{B^{\prime}}\right)=\operatorname{pr}_{\mathcal{H}_{1} \oplus \mathcal{H}_{2}} P\left(g^{\prime}\right)^{*} g^{\prime}=0$. Hence we have a solution to problem (3.14) by

$$
\begin{aligned}
\operatorname{pr}_{\mathcal{H}_{1} \oplus \mathcal{H}_{2}} \circ B\left(\widetilde{g^{\prime \prime}}, \operatorname{pr}_{\mathcal{F}_{1}} \tilde{f}\right) & =0 \quad \text { where } \\
\tilde{f} & :=\varphi^{-1} \circ \bar{\varphi}_{B^{\prime}}^{\text {har }}\left(\left.g^{\prime \prime}\right|_{B^{\prime}}, f \circ P\left(g^{\prime}\right)\right)
\end{aligned}
$$

$\operatorname{as} \operatorname{pr}_{\mathcal{G}^{\circ}} \circ \varphi^{-1} \circ \bar{\varphi}_{B^{\prime}}^{\text {har }}\left(g^{\prime \prime}, f \circ P\left(g^{\prime}\right)\right)=\operatorname{pr}_{\mathcal{G}} \circ \varphi^{-1} \circ \bar{\varphi}_{B^{\prime}}^{\text {har }}\left(g^{\prime \prime}, i d\right)$. Hence $\tilde{P}\left(\widetilde{g^{\prime \prime}}\right)=\operatorname{pr}_{\mathcal{F}_{1}} \tilde{f}$. In other words $\tilde{f}$ and $\tilde{P}\left(\widetilde{g^{\prime \prime}}\right)$ differ only by an element in $\mathcal{F}_{2}$. But by construction this element has no effect on the image under $B$, i.e. $B\left(\widetilde{g^{\prime \prime}}, \tilde{f}\right)=B\left(\widetilde{g^{\prime \prime}}, \tilde{P}\left(\widetilde{g^{\prime \prime}}\right)\right)$. Thus $P\left(\left.g^{\prime \prime}\right|_{B^{\prime}}\right)^{*}\left(\left.g^{\prime \prime}\right|_{B^{\prime}}\right)=\left(f \circ P\left(g^{\prime}\right)\right)^{*}\left(\left.g^{\prime \prime}\right|_{B^{\prime}}\right)=P\left(g^{\prime}\right)^{*} f^{*} g^{\prime \prime}=P\left(g^{\prime}\right)^{*} g^{\prime}$.

\subsection{Proof of the main lemma}

(Note that in this Section Lemma 3.9 is used, see remark preceding this thesis) Finally, we come to the proof of the main Lemma.

Lemma 2.23 (Main Lemma). (Assuming the validity of Lemma 3.9) Given $\Lambda \in \mathbb{R}, i>0, C>0, d \geq 1, \alpha>\beta>0$, and a scale $\varrho \in(0,1)$, for sufficiently small $\Theta>0$ there is a continuous section

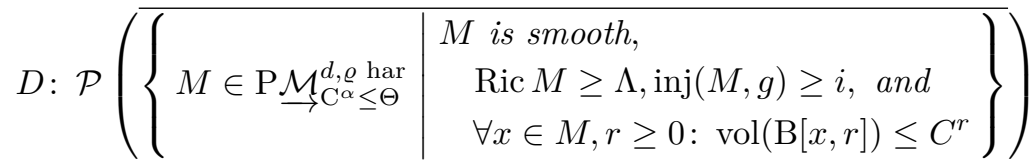

$$
\begin{aligned}
& \rightarrow \mathcal{P}\left(\mathrm{T}_{\mathrm{C}^{\beta} \leq \Theta^{\prime}}^{3,1} \stackrel{\mathrm{P}}{\mathcal{M}_{\mathrm{C}^{\alpha} \leq \Theta}^{d, \varrho}} \underset{\mathrm{har}}{ }\right)
\end{aligned}
$$

for some $\Theta^{\prime}>0$ such that $D(P)$ is a unimodular curvature tensor (see Definition 2.16 and Theorem 2.18).

For brevity write

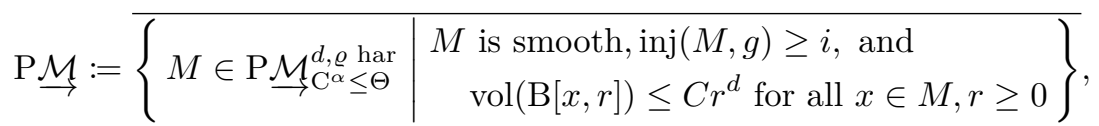

$$
\begin{aligned}
& \operatorname{RP} \mathcal{M}:=\mathrm{T}_{\mathrm{C}^{\beta} \leq \Theta^{\prime}}^{3,1} \stackrel{\mathrm{P}}{\mathcal{M}_{\mathrm{C}^{\alpha}}^{d, \varrho} \leq \Theta} \underset{ }{h a r} .
\end{aligned}
$$

The construction of $D$ is conducted stepwise as summarized in this diagram:

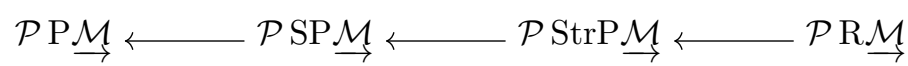

$$
\begin{aligned}
& \overline{\mathfrak{M}} \underset{\text { Step 1 }}{\stackrel{\left.S\right|_{\overline{\mathfrak{M}}}}{\longrightarrow} S(\overline{\mathfrak{M}}) \frac{\cup}{\operatorname{Step~2}} \operatorname{Str} S(\overline{\mathfrak{M}}) \underset{\text { Step 3 }}{\stackrel{\tilde{R}}{\longrightarrow}} D(\overline{\mathfrak{M}})}
\end{aligned}
$$

where the undefined objects and maps will be explained throughout the proof. The three maps above, $S, S t r$, and $D$, will correspond to the three steps of the 
proof. In each step the map is being constructed by Lemma 1.7 and the section property as well as unimodularity are being checked.

For formal transparency of the proof we initially recall and assign all constants involved in terms of $d$ and $\alpha$. Let $\beta, \beta^{\prime}, \beta^{\prime \prime} \in(0,1)$ be such that $\beta<\beta^{\prime}<\beta^{\prime \prime}<\alpha$. Apply Proposition 3.13 to $\alpha=\beta^{\prime}$ obtaining an angle $\varepsilon<\frac{\pi}{2}$ and a norm bound $\bar{\Theta}$. Choose $\Theta<\bar{\Theta}$. Proposition 3.13 also gives a norm bound $\Xi=\Xi(d, \varepsilon, \Theta, r)$. Recall the definition of the following Euclidean ball for $r<\frac{1}{5} e^{-5(\Theta+1)} \varrho$

$$
\begin{array}{ll}
B_{0}:=\mathrm{B}\left[0, e^{-\Theta-1} r\right], & B_{1}:=\mathrm{B}\left[0,2 e^{\Theta+1} r\right], \\
B_{2}:=\mathrm{B}\left[0,4 e^{\Theta+1} r\right], & B_{3}:=\mathrm{B}\left[0,4 e^{3(\Theta+1)} r\right] .
\end{array}
$$

Further let $B_{2}^{\prime}$ and $B_{3}^{\prime}$ be Euclidean balls such that

$$
e^{2(\Theta+1)} B_{2}^{\prime} \subset B_{3}^{\prime} \quad \text { and } \quad e B_{3} \subset B_{2}^{\prime}
$$

Set $\tilde{r}>0$ such that $\tilde{r} \leq \frac{1}{4} e^{-2(\Theta+1)} r$. Finally, let $v$ be the constant from Lemma 2.17 such that $\operatorname{vol}\left(\mathrm{B}\left[x, \frac{1}{3} \tilde{r}\right]\right) \geq v$ for any $M$ with $\|M\|_{\mathrm{C}^{\alpha}, \varrho} \leq \Theta$ and $x \in M$.

To check the section property of Theorem 2.18 more easily, note:

Lemma 3.24. Let $\mathcal{X}$ and $\mathcal{Y}$ be complete separable metric spaces. Further let $m_{(.)}: \mathcal{X} \rightarrow \mathcal{P} \mathcal{Y}$ and $p: \mathcal{Y} \rightarrow \mathcal{X}$ be continuous maps. Let $Q: \mathcal{P} \mathcal{X} \rightarrow \mathcal{P} \mathcal{Y}$ be the integration map induced by Lemma 1.7 and $\eta_{\mathcal{X}}: \mathcal{X} \rightarrow \mathcal{P} \mathcal{X}$ denote the unit of the Giry monad, i.e. the map $x \mapsto \delta_{x}$.

Then $(\mathcal{P} p) \circ m_{(.)}=\eta_{\mathcal{X}}$ implies $(\mathcal{P} p) \circ Q=\operatorname{id}_{\mathcal{P}} \mathcal{X}$.

Proof. Let $f: \mathcal{P} \mathcal{X} \rightarrow \mathbb{R}$ be a bounded continuous test function and $P \in \mathcal{P} \mathcal{X}$. We have $\left.\int f \mathrm{~d}(\mathcal{P} p) \circ Q\right)(P)=\int f \circ p \mathrm{~d} Q(P)=\iint f \circ p \mathrm{~d} m_{x} \mathrm{~d} P(x)=\iint f \mathrm{~d}(\mathcal{P} p) \circ$ $m_{x} \mathrm{~d} P(x)=\int f(x) \mathrm{d} P(x)$.

Assume that $\mathcal{X}$ is a complete separable metric space. Recall that $\mathcal{M} \mathcal{X}$ denotes the space of boundedly finite measures on $\mathcal{X}$, i.e. measures that are finite on bounded sets. Let $\mathcal{N} \mathcal{X} \subset \mathcal{M} \mathcal{X}$ be the space of counting measures on $\mathcal{X}$, i.e. measures with values in $\{0,1, \ldots\} \cup\{\infty\}$. A random (multi-)set of $\mathcal{X}$ is formally a Radon measure on $\mathcal{N} \mathcal{X}$. A point process is a random variable $\Omega \rightarrow \mathcal{N} \mathcal{X}$, this is to say, the distribution law of a point process is a random set. See DV08. Chapter 9] for details.

\subsubsection{First step: random cover}

Recall that a subset $S \subset \mathcal{X}$ of a metric space $\mathcal{X}$ is called $\tilde{r}$-separated if $|x y| \geq \tilde{r}$ for all $x, y \in \mathcal{X}$ and it is called $\tilde{r}$-covering if $\left.S^{\tilde{r}}\right)=\mathcal{X}$.

Definition 3.25. Let $\mathrm{SP} \underset{\mathcal{M}}{\longrightarrow}$ be the subspace (with the restricted topology from Definition 1.28 of isomorphism classes

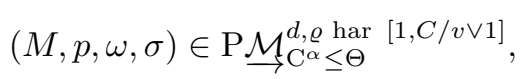

where $\omega$ denotes the orientation, such that

- $\sigma \in \mathcal{N} M$;

- $\sigma$ is $\frac{1}{3} \tilde{r}$-separated; 
- for all $x \in M$ there is an $y \in \sigma \backslash\{x\}$ with $|x y| \leq \tilde{r}$, i.e. $\sigma$ is $\tilde{r}$-covering with respect to closed balls.

Lemma 3.26. The space $\mathrm{SP} \mathcal{M}$ is compact.

Proof. Since the space $\mathrm{P} \mathcal{M}_{\mathrm{C}^{\alpha} \leq \Theta}^{d, \varrho}$ har ${ }^{[1, C / v \vee 1]}$ is compact, it is sufficient to show that $\mathrm{SP} \mathcal{M}$ is closed. Let $\left(M_{n}, p_{n}, \sigma_{n}\right) \in \mathrm{SP} \mathcal{M}$ be a sequence converging to a space $(M, p, \sigma)$. We can find partially comparison maps $\iota_{n}: M_{n} \rightarrow M$ such that $\iota_{n *} \sigma_{n}$ weak $\#$ converges to $\sigma$. Since the $\iota_{n *} \sigma_{n}$ 's are counting measures so is $\sigma$ DV08, Proposition 9.1.IV(iii)]. The second condition can be formulated equivalently by requiring that $\sigma_{n}\left(\mathrm{~B}\left(x, \frac{1}{3} \tilde{r}\right)\right) \leq 1$ for any $x \in \sigma$. As the volume of every bounded open set can only decrease in the limit DV03, Theorem A2.3.II(iii), Proposition A2.6.II(iii)] we have that $\sigma\left(\mathrm{B}\left(x, \frac{1}{3} \tilde{r}\right)\right) \leq 1$. In the same way the volume of bounded closed sets can only increase DV03, Theorem A2.3.II(ii), Proposition A2.6.II(iii)]. And the final condition can equivalently stated as $\sigma_{n}(\mathrm{~B}[x, \tilde{r}]) \geq 1$ and therefore also holds for the limit $\sigma$.

In the same way we define SPM by leaving out orientation. By a random element in SP $\mathcal{M}$ we mean a random variable $X: \Omega \rightarrow$ SP $\mathcal{M}$, where $\Omega=(\Omega, P)$ is a probability space. The probability measure $X_{*}(P)$ is called $\operatorname{Law}(X)$ or distribution of $X$. Bowen [Bow15, Lemma 4.2] proved a lemma that reads in our notation (stated for Riemannian manifolds) as follows:

Lemma 3.27. Let $\tilde{r}>0$. There exists a continuous map

$$
m_{(.)}: \mathrm{PM}_{\mathrm{C}^{\alpha} \leq \Theta}^{d, \varrho \text { har }} \rightarrow \mathcal{P} \mathrm{SPM}
$$

such that,

(i) for any $(M, p) \in \mathrm{P} \mathcal{M}_{\mathrm{C}^{\alpha} \leq \Theta}^{d, \varrho \text { har }}$ we have that, if $\left(M^{\prime}, p^{\prime}, \sigma^{\prime}\right) \in \mathrm{SP} \mathcal{M}$ is random with $\operatorname{Law}\left(\left(M^{\prime}, p^{\prime}, \sigma^{\prime}\right)\right)=m_{(M, p)}$, then $\left(M^{\prime}, p^{\prime}\right)=(M, p)$ and $\sigma^{\prime}$ is $\frac{1}{3} \tilde{r}_{-}$ separated and $\tilde{r}$-covers $M$ almost surely.

(ii) Moreover, $m_{(.)}$does not depend on the point $p$ in the following sense: If $(M, p),(M, q)$ are pointed spaces in $\mathrm{P}_{\mathrm{C}^{\alpha} \leq \Theta}^{d, \varrho}$ har and $(M, p, \sigma),\left(M, p, \sigma^{\prime}\right)$ random with $\operatorname{Law}((M, p, \sigma))=m_{(M, p)}$, $\operatorname{Law}\left(\left(M, q, \sigma^{\prime}\right)\right)=m_{(M, q)}$, then $\operatorname{Law}(\sigma)=\operatorname{Law}\left(\sigma^{\prime}\right)$.

Observe that for a representative of an equivalence class $(M, p, s) \in \mathrm{P} \mathcal{M}$, also denoted by $(M, p, s)$, where $s$ is the orientation, two cases can happen: First there is an orientation reversing pointed isomorphism $f:(M, p) \rightarrow(M, p)$ or not. The former means that the spaces possesses an orientation reversing symmetry. More precisely, we can say:

Lemma 3.28. The subset of $M \in \mathrm{P} \underline{\mathcal{M}}$ admitting an orientation reversing pointed isomorphism is closed.

Proof. Take a sequence of spaces $M_{n} \in \mathrm{P} \underline{\mathcal{M}}$ with orientation reversing pointed isomorphisms $f_{n}: M_{n} \rightarrow M_{n}$. Moreover choose orientation preserving comparison maps $\iota_{n}: M_{n} \rightarrow M$ for some radius $r$ such that $\mathrm{B}[p, r] \subset \operatorname{im}\left(\iota_{n}\right)$ and $\mathrm{B}\left[p_{n}, r\right] \subset \operatorname{dom}\left(\iota_{n}\right)$. This gives approximate pointed orientation reversing maps $f_{r, n}:(\mathrm{B}[p, r], p) \rightarrow(M, p)$ defined by $\iota \circ f_{n} \circ \iota^{-1}$, meaning that $\inf _{\mathrm{B}[p, r]}\left|f_{r, n}^{*} s\right|_{g} \geq 1$ 
and $f_{r, n}^{*} s$ represents the opposite orientation. Application of Arzelà-Ascoli gives a convergent subsequence. Repeating this argument for a sequence $r_{n} \rightarrow \infty$ and a diagonal argument gives a globally defined orientation preserving pointed isomorphism $f: M \rightarrow M$.

First Step of Proof of Lemma 2.23. First, we refine Bowen's map $m_{(.)}$to a continuous map

$$
m_{(.)}^{S}: \mathrm{P} \stackrel{\mathcal{M}}{\rightarrow} \mathcal{P} \text { SP } \stackrel{\mathcal{M}}{\longrightarrow}
$$

For any $(M, p) \in \mathrm{P} \mathcal{M}$ let $X_{(M, p)}: \Omega \rightarrow$ SP $\mathcal{M}$ be a random variable with law $m_{(M, p)}$ on some probability space $\Omega=\left(\Omega, P_{\Omega}\right)$. For a space $(M, p, s) \in \mathrm{P} \underline{\mathcal{M}}$ define the random variable $Y_{(M, p, s)}: \Omega \rightarrow \mathcal{P}(\mathrm{SP} \underline{\mathcal{M}})$ by

$$
Y_{(M, p, s)}(\omega):=\left\{\begin{array}{cr}
\delta_{\left(X_{(M, p)}(\omega), s\right)} & \text { if } \nexists \text { orientation reversing } \\
\frac{1}{2} \delta_{\left(X_{(M, p)}(\omega), s\right)} & \text { pointed isomorphism } \\
+\frac{1}{2} \delta_{\left(X_{(M, p)}(\omega), f s\right)} & \text { else, } f \text { such an isomorphism }
\end{array}\right.
$$

(where by abuse of notation we write $((M, p, \sigma), s)=(M, p, \sigma, s)$ ) which is well defined since in the first case there is no ambiguity of how to assign the orientation and in the second case all choices are made simultaneously, i.e. there is no dependence on $f$. Moreover, $Y_{(M, p, s)}$ is measurable since the case distinction is based on a closed condition by Lemma 3.28 As the definition does not depend on $\omega$ but only on $X_{(M, p)}(\omega)$, the law of $Y_{(M, p, s)}$ is independent of the choice of $X_{(M, p)}$. Thus define

$$
m_{(M, p, s)}^{S}:=\mu_{\operatorname{SP} \underset{\mathcal{M}}{\rightarrow}}\left(\operatorname{Law} Y_{(M, p, s)}\right)
$$

where $\mu_{\mathrm{SP}} \underline{\mathcal{M}}: \mathcal{P}(\mathcal{P}(\mathrm{P} \underline{\mathcal{M}})) \rightarrow \mathcal{P}(\mathrm{P} \underline{\mathcal{M}})$ is the counit of the Giry monad, see Lemma $\overrightarrow{1.8}$, i.e. the integration $\mu_{\mathrm{SP}} \underset{\mathcal{M}}{\rightarrow}(P)(A)=\int P^{\prime}(A) \mathrm{d} P\left(P^{\prime}\right)$ for some Borel set $A \subset \mathrm{PM}$.

Continuity of $m^{S}$. Take a sequence $M_{n}=\left(M_{n}, p_{n}, s_{n}\right)$ converging to $M=$ $(M, p, s)$ in P $\mathcal{M}$. By standard probability theory [Dud02, Theorem 11.7.1(d)] we can choose random variables $X_{M_{n}, p_{n}}, X_{(M, p)}: \Omega \rightarrow \mathrm{SP} \mathcal{M}$ with laws such that $X_{\left(M_{n}, p_{n}\right)}(\omega)$ a.s. converges to $X_{(M, p)}(\omega)$. Let $Y_{n}:=Y_{\left(M_{n}, p_{n}, s_{n}\right)}$ and $Y:=Y_{\left(M_{, p}, s\right)}$ be random variables defined by 3.15 . To show that $m_{\left(M_{n}\right)}^{S}$ converges to $m_{(M)}^{S}$, we show that $Y_{n}(\omega)$ a.s. converges to $Y(\omega)$. In the first case assume that $M$ admits an orientation reversing pointed isomorphism. Moreover let $\bar{s}_{n}$ denote the reversed orientation of $M_{n}$ and $f$ an orientation reversing pointed isomorphism of $M$. Then any comparison maps $\iota_{n}:\left(M_{n}, p_{n}, s_{n}\right) \rightarrow M$ give also comparison maps $f \circ \iota_{n}:\left(M_{n}, p_{n}, \bar{s}_{n}\right) \rightarrow M$. As we are in a metrizable space, for some chosen metric $d$ we have $d\left(\left(M_{n}, p_{n}, s_{n}\right),\left(M_{n}, p_{n}, \bar{s}_{n}\right)\right) \rightarrow 0$ as $n \rightarrow \infty$. Hence both laws

$$
\delta_{X_{\left(M_{n}, p_{n}\right)}, s} \text { and } \quad \frac{1}{2} \delta_{\left(X_{\left(M_{n}, p_{n}\right)}, s\right)}+\frac{1}{2} \delta_{\left(X_{\left(M_{n}, p_{n}\right)}, \bar{s}\right)}
$$

converge to each other as $n \rightarrow \infty$. In the second case assume that $M$ does not admit an orientation preserving pointed isomorphism. Then, as by Lemma 3.28 the set of such $M$ is open, for sufficiently large $n$ also $M_{n}$ does not admit such 
an isomorphism. Hence by 3.15 we have $Y=\delta_{\left(X_{(M, p)}(\omega), s\right)}$. Any comparison maps $\iota_{n}:\left(M_{n}, p_{n}, s_{n}\right) \rightarrow M$ give directly comparison maps $\left(Y_{n}(\omega), s_{n}\right) \rightarrow$ $\left(X_{(M, p)}(\omega), s\right)$. This proves convergence $Y_{n}(\omega) \rightarrow Y(\omega)$ a.s.

The map $S: \mathcal{P} P \stackrel{\mathcal{M}}{\rightarrow} \rightarrow \mathcal{P}(\mathrm{SP} \stackrel{\mathcal{M}}{\rightarrow})$ is now given by $m^{S}$ and the integration lemma, Lemma 1.7

Unimodularity of $S$. Observe that for any unimodular law $P$ on $\mathrm{P} \mathcal{M}$ and for any bounded test function with bounded support $\varphi: \operatorname{SPP} \underline{\mathcal{M}} \rightarrow \mathbb{R}$ on doubly pointed spaces with random subset carrying the metric defined in 1.25 we have

$$
\begin{aligned}
& \int \varphi(M, p, q, s, \sigma) \mathrm{d} m_{\mathrm{L}}(S(P)) \\
&=\iint_{M} \varphi(M, p, q, s, \sigma) \mathrm{d} \operatorname{vol}_{M}(q) \mathrm{d}(S P)(M, p, s, \sigma) \\
&=\iint_{M} \varphi(M, p, q, s, \sigma) \mathrm{d} \operatorname{vol}_{M}(q) \mathrm{d} m_{\left(M^{\prime}, p^{\prime}, s^{\prime}\right)}^{S}(M, p, s, \sigma) \mathrm{d} P\left(M^{\prime}, p^{\prime}, s^{\prime}\right) \\
&=\iint_{\Omega} \int_{M} \varphi(M, p, q, s, \sigma) \mathrm{d} \operatorname{vol}_{M}(q) \mathrm{d} Y_{\left(M^{\prime} p^{\prime}, s^{\prime}\right)}(\omega)(M, p, s, \sigma) \\
& \mathrm{d} P_{\Omega}(\omega) \mathrm{d} P\left(M^{\prime}, p^{\prime}, s^{\prime}\right)
\end{aligned}
$$

write $X(\omega)=\left(M_{\omega}, p_{\omega}, s_{\omega}, \sigma_{\omega}\right)$, and do the case distinction from 3.15

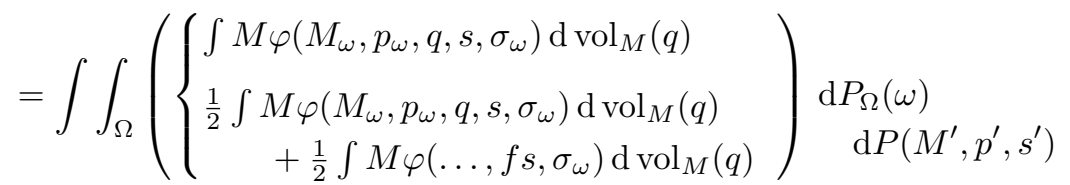

where $f$ is the change of orientation. In the integrand we can consider $M, p$, and $s$ as fixed, i.e. $\varphi(M, p, q, s, \sigma)=\varphi_{(M, p, s)}(M, q, \sigma)$. Thereby we can interchange integration due to independence of $\sigma_{\omega}$ from the point as stated in part (ii) of Bowen's Lemma 3.27.

$$
\begin{aligned}
& =\iint_{M} \int_{\Omega}\left(\begin{array}{l}
\varphi\left(M_{\omega}, p_{\omega}, q, s, \sigma_{\omega}\right) \\
\frac{1}{2} \varphi\left(M_{\omega}, p_{\omega}, q, s, \sigma_{\omega}\right)+\ldots
\end{array}\right) \begin{array}{r}
\mathrm{d} \mathrm{vol}_{M}(q) \\
\mathrm{d} P_{\Omega}(\omega) \mathrm{d} P\left(M^{\prime}, p^{\prime}, s^{\prime}\right)
\end{array} \\
& =\iint_{M} \int_{\Omega}\left(\left\{\begin{array}{l}
\varphi\left(M_{\omega}, p_{\omega}, q, s, \sigma_{\omega}\right) \\
\frac{1}{2} \varphi\left(M_{\omega}, p_{\omega}, q, s, \sigma_{\omega}\right)+\ldots
\end{array}\right) \begin{array}{r}
\mathrm{d} P_{\Omega}(\omega) \\
\mathrm{d} \mathrm{vol}_{M}(q) \mathrm{d} P\left(M^{\prime}, p^{\prime}, s^{\prime}\right)
\end{array}\right.
\end{aligned}
$$

since $X_{n}$ depends only on the isomorphism class

$$
=\iint_{M} \int_{\Omega}\left(\begin{array}{l}
\varphi\left(M_{\omega}, p_{\omega}, q, s, \sigma_{\omega}\right) \\
\frac{1}{2} \varphi\left(M_{\omega}, p_{\omega}, q, s, \sigma_{\omega}\right)+\ldots
\end{array}\right) \mathrm{d} P_{\Omega}(\omega) \mathrm{d} m_{\mathrm{L}} P\left(M^{\prime}, p^{\prime}, q, s^{\prime}\right)
$$

using unimodularity of $P$

$$
\begin{aligned}
& =\iint_{M} \int_{\Omega}\left(\begin{array}{l}
\varphi\left(M_{\omega}, p_{\omega}, q, s, \sigma_{\omega}\right) \\
\frac{1}{2} \varphi\left(M_{\omega}, p_{\omega}, q, s, \sigma_{\omega}\right)+\ldots
\end{array}\right) \mathrm{d} P_{\Omega}(\omega) \mathrm{d} m_{\mathrm{R}} P\left(M^{\prime}, p^{\prime}, q, s^{\prime}\right) \\
& =\iint_{M} \int_{\Omega}\left(\left\{\begin{array}{l}
\varphi\left(M_{\omega}, p_{\omega}, q, s, \sigma_{\omega}\right) \\
\frac{1}{2} \varphi\left(M_{\omega}, p_{\omega}, q, s, \sigma_{\omega}\right)+\ldots
\end{array}\right) \begin{array}{r}
\mathrm{d} P_{\Omega}(\omega) \\
\mathrm{d} \mathrm{vol}_{M}\left(p^{\prime}\right) \mathrm{d} P\left(M^{\prime}, q, s^{\prime}\right)
\end{array}\right.
\end{aligned}
$$


using again idependence of the base point

$$
\begin{aligned}
& =\iint_{M} \int_{\Omega}\left(\begin{array}{l}
\varphi\left(M_{\omega}, p^{\prime}, q_{\omega}, s, \sigma_{\omega}\right) \\
\frac{1}{2} \varphi\left(M_{\omega}, p^{\prime}, q_{\omega}, s, \sigma_{\omega}\right)+\ldots
\end{array}\right) \begin{array}{r}
\mathrm{d} P_{\Omega}(\omega) \\
\mathrm{d} \operatorname{vol}_{M}\left(p^{\prime}\right) \mathrm{d} P\left(M^{\prime}, q, s^{\prime}\right)
\end{array} \\
& =\iint_{\Omega} \int_{M} \varphi\left(M, p^{\prime}, q^{\prime}, s, \sigma\right) \mathrm{d} \operatorname{vol}_{M}\left(p^{\prime}\right) \mathrm{d} Y_{\left(M^{\prime}, q, s^{\prime}\right)}(\omega)\left(M, q^{\prime}, s, \sigma\right) \\
& =\iint_{M} \varphi\left(M, p^{\prime}, q^{\prime}, s, \sigma\right) \mathrm{d} \operatorname{vol}_{M}\left(p^{\prime}\right) \mathrm{d} m_{\left(M^{\prime}, q, s^{\prime}\right)}^{S}\left(M, q^{\prime}, s, \sigma\right) \mathrm{d} P\left(M^{\prime}, q, s^{\prime}\right) \\
& =\iint_{M} \varphi(M, p, q, s, \sigma) \mathrm{d} \operatorname{vol}_{M}(p) \mathrm{d}(S P)(M, q, s, \sigma) \\
& =\int \varphi(M, p, q, s, \sigma) \mathrm{d} m_{\mathrm{R}}(S P) \text {. }
\end{aligned}
$$

This proves $m_{\mathrm{L}}(S P)=m_{\mathrm{R}}(S P)$, i.e. unimodularity.

Section property. It is sufficient according to Lemma 3.24 to check for any point $(M, p, s) \in \mathrm{P} \mathcal{M}$ and any bounded test function with bounded support $\varphi: \mathrm{P} \underline{\mathcal{M}} \rightarrow \mathbb{R}$ on doubly pointed spaces that

$$
\begin{aligned}
\int \varphi & \mathrm{d} m^{S}(M, p, s) \\
& =\iint \varphi \mathrm{d} P^{\prime} \mathrm{d}\left(\operatorname{Law} Y_{(M, p, s)}\right)\left(P^{\prime}\right) \\
& =\int_{\Omega}\left(\left\{\begin{array}{l}
\int \varphi \mathrm{d} \delta_{\left(X_{(M, p)}(\omega), s\right.} \varphi \mathrm{d} \frac{1}{2} \delta_{\left(X_{(M, p)}(\omega), s\right)}+\frac{1}{2} \delta_{\left(X_{(M, p)}, f s\right)}
\end{array}\right) \mathrm{d} P_{\Omega}(\omega)\right. \\
& =\int_{\Omega}\left(\left\{\begin{array}{l}
\varphi\left(X_{(M, p)}(\omega), s\right) \\
\frac{1}{2} \varphi\left(X_{(M, p)}(\omega), s\right)+\frac{1}{2} \varphi\left(X_{(M, p)}, f s\right)
\end{array}\right) \mathrm{d} P_{\Omega}(\omega) \mathrm{d} P(M, p, s)\right.
\end{aligned}
$$

where by abuse of notation we write $\varphi((M, p, \sigma), s)$ instead of $\varphi(M, p, s)$. According to the section property contained in Bowen's Lemma 3.27 part (i), this is

$$
=\left\{\begin{array}{l}
\varphi(M, p, s) \\
\frac{1}{2} \varphi(M, p, s)+\frac{1}{2} \varphi(M, p, f s)
\end{array}\right.
$$

in the second case by definiton of the quotient space $\mathrm{P} \underset{\mathcal{M}}{\rightarrow}$ we have $(M, p, s)=$ $(M, p, f s)$. Hence

$$
=\varphi(M, p, s)=\int \varphi \mathrm{d} \delta_{(M, p, s)} .
$$

\subsubsection{Second Step: random strainer}

Let $\Delta: x \mapsto(x, x)$ be the diagonal map from $M$ to $M^{2}$. Endow $M^{2}=M \times M$ with the metric $\left|\left(x, x^{\prime}\right)\left(y, y^{\prime}\right)\right|:=\frac{1}{2}\left(|x y|+\left|x^{\prime} y^{\prime}\right|\right)$ The idea is to assign to a space 
$(M, p, \nu) \in \mathrm{SP} \underline{\mathcal{M}}$ with $M=(M, g$, vol,$s)$ the space

$$
\begin{aligned}
\left(M^{2}, \Delta_{*} v o l, \sigma \times v o l,\right. & \sum_{x \in \sigma} \delta_{\left(x, z_{x 1}^{+}\right)}, \ldots, \sum_{x \in \sigma} \delta_{\left(x, z_{x d}^{+}\right)} \\
& \left.\sum_{x \in \sigma} \delta_{\left(x, z_{x 1}^{-}\right)}, \ldots, \sum_{x \in \sigma} \delta_{\left(x, z_{x d}^{-}\right)}\right)
\end{aligned}
$$

such that $\left(z_{x 1}^{+}, \ldots, z_{x d}^{+}, z_{x 1}^{-}, \ldots, z_{x d}^{-}\right)$is an $\varepsilon$-strainer of $x$ contained in $\mathrm{B}[x, 2 r] \backslash$ $\mathrm{B}(p, r)$ for each point $x \in \sigma$. This way of encoding the strainers can be visualized by:

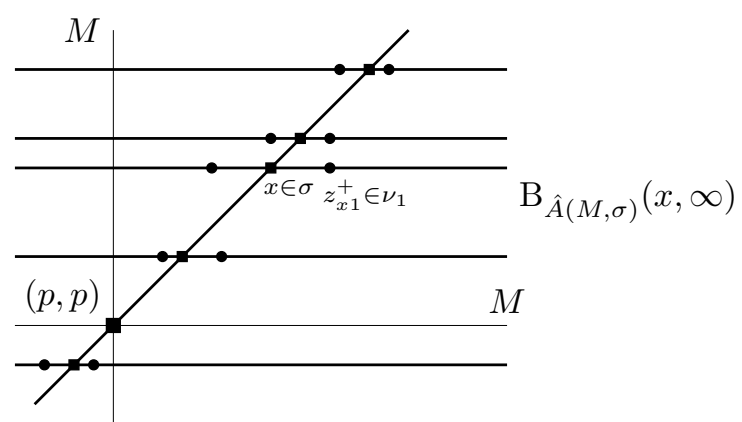

Recall that for a subspace $A \subset \mathcal{X}$ of some metric space $\mathcal{X}$ we denote by $\widehat{A}$ the set $A$ endowed with the intrinsic metric 1.6 of the restriction of the metric of $\mathcal{X}$ to $A \times A$. For a measured space $(\mathcal{X}, \mu)$ and a measurable set $A \subset \mathcal{X}$ denote by $\mu \cap A$ the restriction of $\mu$ to $A$. Set $A(M, \sigma):=\operatorname{supp}(\sigma \times v o l)$ and $\hat{A}(M, \sigma):=\widehat{A(M, \sigma)}$.

Definition 3.29. Let $\operatorname{StrP} \stackrel{\mathcal{M}}{\longrightarrow}$ be the subspace of isomorphism classes

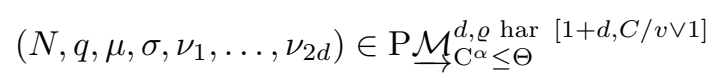

(where we also write $\vec{\nu}=\nu_{1}, \ldots, \nu_{2 d}$ ) such that there is a Riemannian manifold $M \in \mathrm{P} \underline{\mathcal{M}}$ with an mm-space isomorphism $\varphi:\left(M \times M,(p, p), \Delta_{*} v o l\right) \rightarrow(N, q, \mu)$ with

- $(\operatorname{supp} \mu) \cap \sigma$ is a $\frac{1}{3} \tilde{r}$-separated set of $M=\Delta(N) \subset N$;

- $(\operatorname{supp} \mu) \cap \sigma$ is a $\tilde{r}$-covering set of $M$ with respect to closed balls, i.e. in the sense $((\operatorname{supp} \mu) \cap \sigma)^{\tilde{r}]}=M$;

- $\nu_{1}, \ldots, \nu_{2 d} \in \mathcal{N} N$ and $\operatorname{supp}\left(\nu_{1}\right), \ldots, \operatorname{supp}\left(\nu_{2 d}\right) \subset \hat{A}(M, \sigma)$;

- for any $x \in(\operatorname{supp} \mu) \cap \sigma$ the sets $\nu_{1} \cap \mathrm{B}_{\hat{A}(M, \sigma)}(x, \infty), \ldots, \nu_{2 d} \cap \mathrm{B}_{\hat{A}(M, \sigma)}(x, \infty)$ are singletons, their elements form an $\varepsilon$-strainer of $x$ in $M$.

Lemma 3.30. The subspace $\operatorname{Str} P \underset{\mathcal{M}}{\longrightarrow} \subset \mathrm{P} \underset{\mathcal{M}_{\mathrm{C}^{\alpha}}^{d, \varrho} \text { har }}{[1+d, C / v \vee 1]}$ is compact.

Proof. Given a sequence $N_{n} \in \operatorname{StrP} \underline{\mathcal{M}}$. Choose representations $M_{n}$ with mmspace isometries $\varphi_{n}:\left(M_{n} \times M_{n},\left(p_{n}, \overrightarrow{p_{n}}\right), \Delta_{*} v l_{n}\right) \rightarrow\left(N_{n}, q_{n}, \mu_{n}\right)$. Note that by assumption we can reconstruct the restrictions of the additional measures $\sigma \cap M_{n}$ and $\vec{\nu}$ from the measures $(\operatorname{supp} \mu) \cap \sigma$ and $\nu_{n 1} \cap \mathrm{B}_{\hat{A}(M, \sigma)}(x, \infty), \ldots, \nu_{n(2 d)} \cap$ 
$\mathrm{B}_{\hat{A}(M, \sigma)}(x, \infty)$, for $x \in(\operatorname{supp} \mu) \cap \sigma$, viewed as measures on $M$ by formula (3.16).

By compactness of $\mathrm{P} \mathcal{M}$ the $M_{n}$ 's subconverge to some $M$. All the additional measures $\left(\operatorname{supp} \mu_{n}\right) \cap \sigma_{n}$ and $\nu_{n 1} \cap \mathrm{B}_{\hat{A}(M, \sigma)}(x, \infty), \ldots, \nu_{n(2 d)} \cap \mathrm{B}_{\hat{A}(M, \sigma)}(x, \infty)$, for $x \in(\operatorname{supp} \mu) \cap \sigma \cap B \times M$, viewed as measures on $M_{n}$ can be pushed forward to $M$ after choice of partially defined comparing maps $\iota_{n}: M_{n} \rightarrow M$ as given by Lemma A.8, i.e. for any $r>0$ and sufficiently large $n$ the ball $\mathrm{B}(p, r)$ is contained in the image of $\iota_{n}$ and orientation, distance, and volume converge with respect to the $\iota_{n}$ 's. Since - for some constant $C$ - the space of measures $\mu \in \mathcal{M} M$ subject to

$$
\mu(\mathrm{B}[x, r]) \leq C r^{d}+C
$$

is compact DV03 Corollary A2.6.V], all these push forward measures subconverge to some measures on $M$. Hence we can find a converging subsequence of the $M_{n}$ 's.

Observe that for any open ball $B \subset M$ the collection of $\operatorname{spaces}(N, q, \mu, \sigma$, $\left.\nu_{1}, \ldots, \nu_{2 d}\right)$ such that all condition in Definition 3.29 hold on $B$ is closed: The $\frac{1}{3} \tilde{r}$-separating and $\tilde{r}$-covering conditions obviously hold for the limit measures. The counting measures $\vec{\nu}_{n}$ converge to counting measures DV08, Proposition 9.1.IV(iii)]. More precisely, since the sets $\nu_{n 1} \cap \mathrm{B}_{\hat{A}(M, \sigma)}(x, \infty), \ldots, \nu_{n(2 d)} \cap$ $\mathrm{B}_{\hat{A}(M, \sigma)}(x, \infty)$, for $x \in(\operatorname{supp} \mu) \cap \sigma \cap B \times M$, are singletons their push forward measures converge to points. Hence convergence of these measures just means convergence of points. The $\varepsilon$-strainer conditions closed as well, so it holds for the limit points.

Since the condition holds for any closed ball $B$ and arbitrary intersections of closed balls are closed, the collection of spaces $\left(N, q, \mu, \sigma, \nu_{1}, \ldots, \nu_{2 d}\right)$ such that all conditions in Definition 3.29 hold is closed. This is to say that every limit of the subconverging sequence $M_{n}$ is in $\operatorname{StrP} \underline{\mathcal{M}}$.

Lemma 3.31 (lifting lemma). Let $\mathcal{X}$ and $\mathcal{Y}_{1}, \mathcal{Y}_{2}, \ldots$ be Polish spaces and $\mu_{1}, \ldots$ laws on $\mathcal{Y}_{1}, \ldots$ with compact support and $f_{n}: \mathcal{Y}_{n} \rightarrow \mathcal{X}$ be measurable maps. For $n=1, \ldots$ let $X_{n}: \Omega \rightarrow \mathcal{X}$ be random variables with law $f_{n *} \mu_{n}$. Then there is a probability space $\Omega^{\prime}=\left(\Omega^{\prime}, P^{\prime}\right)$ with random variables $Y_{n}: \Omega^{\prime} \rightarrow \mathcal{Y}_{n}$, for $n=1, \ldots$, such that $X_{n}=f_{n} \circ Y_{n}$ a.s. and Law $Y_{n}=\mu_{n}$.

Proof. Take a disintegration $\left\{P_{n x}\right\}_{x \in X}$ of each $f_{n *} \mu_{n}$ AGS08, Theorem 5.3.1], i.e. a family of probability measures $\left\{P_{n x}\right\}_{x \in X}$ such that $\mu_{x}\left(f_{n}^{-1}(x)\right)=1$ and integration with respect to $\mu_{n}$ is expressed by $\iint \varphi(y) \mathrm{d} P_{x}(y) \mathrm{d} f_{n *} \mu_{n}(x)$. Set $\Omega^{\prime}:=\Omega \times \prod_{n=1}^{\infty} M_{n}$ with probability measure $P^{\prime}$ defined by integration Fre03 $452 \mathrm{~B}(\mathrm{~b})(\mathrm{i})]$ of the family of probability measures

$$
\left(\delta_{\omega} \times \prod_{n=1}^{\infty} P_{n X_{n}(\omega)}\right)_{\omega \in \Omega}
$$

with respect to the projection $\Omega^{\prime} \rightarrow \Omega$. For $n=1, \ldots$ set $Y_{n}: \Omega^{\prime} \rightarrow M_{n}$ to be the projection to $M_{n}$. First observe that for every $n$ it holds true $P^{\prime}$-a.s. that for any $\left(\omega, x_{1}, \ldots\right) \in \Omega^{\prime}$ we have $x_{n} \in \operatorname{supp} P_{n X_{n}(\omega)}$ and hence $f_{n}\left(Y_{n}(\omega)\right)=X_{n}(\omega)$. Moreover for any bounded real valued function $\varphi: \mathcal{Y}_{n} \rightarrow \mathbb{R}$ observe that $\int \varphi \mathrm{d} \mu_{n}=$ $\iint \varphi(y) \mathrm{d} P_{n x}(y) \mathrm{d} f_{n *} \mu_{n}(x)=\iint \varphi(y) \mathrm{d} P_{n X_{n}(\omega)}(y) \mathrm{d} P(\omega)=\int \varphi\left(Y_{n}\right) \mathrm{d} P^{\prime}$. This proves also Law $Y_{n}=\mu_{n}$. 
Second Step of Proof of Lemma 2.23. We construct a lift from $S(\overline{\mathfrak{M}})$ to the space $\mathcal{P} \operatorname{StrP} \mathcal{M}$. Again we construct this map $S t r$ by integration according to Lemma 1.7 Define

$$
\begin{gathered}
f_{x}: \vec{z} \mapsto 0 \vee \min _{\substack{i \neq j \\
i, j=1, \ldots, d}}\left\{\begin{array}{r}
\varepsilon-\left|\widetilde{\measuredangle} z_{i}^{+} x z_{j}^{+}-\frac{\pi}{2}\right|, \varepsilon-\left|\widetilde{\measuredangle} z_{i}^{+} x z_{i}^{-}-\pi\right|, \\
\frac{1}{2} r-|| z_{i} x\left|-\frac{3}{2} r\right|
\end{array}\right\}, \\
M^{\times 2 d} \rightarrow[0, \infty)
\end{gathered}
$$

with $\vec{z}=\left(z_{1}^{+}, \ldots, z_{d}^{+}, z_{1}^{-}, \ldots, z_{d}^{-}\right)$. As vol is fully supported for any $M \in S(\overline{\mathfrak{M}})$, the support of $f_{x}$ has always positive measure. Hence we can normalize $f_{x}$ by

$$
\bar{f}_{x}:=\frac{1}{\int f_{x} \mathrm{~d} v o l} f_{x} .
$$

that is a non-negative bounded function that does not vanish only if $\vec{z}$ is an $\varepsilon$-strainer for $x$ located in $\mathrm{B}(x, 2 r) \backslash \mathrm{B}[x, r]$. Fact $(1.5)$ in the special case of a constant sequence $M=M_{n}$ proves continuity of $f_{x}$. Now we define the integrand function $m^{\text {Str }}: \operatorname{SP} \mathcal{M} \rightarrow \mathcal{P} \operatorname{StrP} \mathcal{M}$. Set

$$
\tilde{m}_{(M, p, \sigma)}^{S t r}:=i_{*}\left(\sum_{x \in \sigma} \delta_{x}^{2 d} \times\left(\eta_{M}\right)_{*} \bar{f}_{x} v o l^{2 d}\right),
$$

where $\eta_{M}$ is the unit of the Giry monad from Lemma 1.8 i.e. $M \rightarrow \mathcal{P} M, x \mapsto \delta_{x}$, and $i_{*}$ is the index shuffle $\left(x_{1}, \ldots, x_{2 d}, y_{1}, \ldots, y_{2 d}\right) \mapsto\left(x_{1}, y_{1}, \ldots, x_{2 d}, y_{2 d}\right)$. This map is invariant under representatives of equivalence classes in SP $\underline{\mathcal{M}}$ and change of base point, i.e.

$$
\begin{aligned}
\tilde{m}_{\left(M^{\prime}, p^{\prime}, \sigma^{\prime}\right)}^{S t r} & =f_{*} \tilde{m}_{(M, p, \sigma)}^{S t r}, \quad \text { and } \\
\tilde{m}_{(M, q, \sigma)}^{S t r} & =\tilde{m}_{(M, p, \sigma)}^{S t r}
\end{aligned}
$$

for any isomorphism $f:(M, p, \sigma) \rightarrow\left(M^{\prime}, p^{\prime}, \sigma^{\prime}\right)$ and any points $p, q \in M$. Finally,

$$
m_{(M, p, \nu)}^{S t r}:=\left(\vec{\nu} \mapsto\left(M \times M,(p, p), \operatorname{vol} \times \delta_{p}, \nu \times \operatorname{vol}, \vec{\nu}\right)\right)_{*} \tilde{m}_{(M, p, \nu)}^{S t r}
$$

Continuity of $m^{S t r}$. Let $\left(M_{n}, p_{n}, \sigma_{n}\right) \in \mathrm{SP} \mathcal{M}$ converging to some $M \in \mathrm{SPM}$ via some $\iota_{n}: M_{n} \rightarrow M$. Let $X_{n}, X: \Omega \rightarrow \overrightarrow{\mathrm{SP}} \stackrel{\mathcal{M}}{\rightarrow}$ be random variables with laws $m_{\left(M_{n}, p_{n}, \nu_{n}\right)}^{S t r}, m_{(M, p, \nu)}^{S t r}$. Write $X=\left(M \times \overrightarrow{M,} \mu, \nu, \vec{\nu}^{\omega}\right)$ and $X_{n}=\left(M_{n} \times\right.$ $\left.M_{n}, \mu_{n}, \nu_{n}, \vec{\nu}_{n}^{\omega}\right)$.

For a radius $s \geq 0$ set

$$
\begin{aligned}
Y^{(s)}(\omega) & :=X(\omega) \cap \mathrm{B}(p, s) \\
& =\left(M \times M, \mu, \sigma \cap \mathrm{B}(p, s)^{2}, \vec{\nu}^{\omega} \cap(\mathrm{B}(p, s) \times M)^{d} ;\right.
\end{aligned}
$$

for some $\vec{\nu}^{(\cdot)}: \Omega \rightarrow(\mathcal{N} M)^{\times(2 d)}$; this is well-defined as it can be rephrased in a way invariant under pointed isometries $M \times M \rightarrow M \times M$, namely

$$
\vec{\nu} \cap(\mathrm{B}(p, s) \times M)=\nu \cap \mathrm{B}_{\hat{A}(M, \sigma)}\left(\mathrm{B}_{\operatorname{supp} \mu}(p, s), \infty\right) .
$$

Define $Y_{n}^{(r)}(\omega)=X_{n}^{(r)}(\omega) \cap \mathrm{B}\left(p_{n}, s\right)$ in the same way. 
By definition of convergence in $\operatorname{StrP\mathcal {M}}$ we know that $Y_{n}^{s}$ converges in law to $X_{n}$ a.s. as $s \rightarrow \infty$ and $Y^{s}$ converges to $X$ in law a.s. as $s \rightarrow \infty$. Since $\mathcal{M}$ is metrizable this implies that to show convergence $X_{n} \rightarrow X$ in law it is sufficient to show convergence

$$
Y_{n}^{(s)} \rightarrow Y^{(s)}
$$

in law for each $s>0$.

As the elements of $\sigma$ are $\frac{1}{3} \tilde{r}$-separated, there is, for sufficiently large $n$, a unique point $x_{n} \in \sigma_{n}$ with $\left|x \iota_{n}\left(x_{n}\right)\right|<\frac{1}{6} \tilde{r}$. For the $\iota_{n}$ 's we know that $\left(\iota_{n}^{d}\right)_{*} \bar{f}_{x_{n}} v o l_{n}^{2 d} \rightarrow \bar{f}_{x} v o l^{2 d}$.

For each $x \in \sigma$ choose random variables $X_{x n}, X_{x}: \Omega \rightarrow M$ with laws

$$
\text { Law } X_{x n}=\left(\iota_{n}^{d}\right)_{*} \bar{f}_{x_{n}} v o l_{n}^{2 d} \quad \text { and } \quad \text { Law } X_{x}=\bar{f}_{x} v^{2 d} l^{2 d}
$$

such that $X_{x n} \rightarrow X_{x}$ a.s. By Lemma 3.31 (applied to $\mathcal{X}=M^{2 d}$ and $\mathcal{X}_{n}=M_{n}^{2 d}$ ) we can assume that there is a lift of $X_{x n}$ to a random variable $Y_{x n}: \Omega \rightarrow M_{n}$, i.e. we have

$$
\text { Law } Y_{n}=\bar{f}_{x_{n}} \text { vol }_{n}^{2 d} \quad \text { and } \quad \iota_{n} \circ Y_{n}=X_{n} \quad \text { a.s. }
$$

Choose

$$
\begin{aligned}
Y_{n}^{(s)} & :=\left(M \times M,(p, p), v o l \times \delta_{p}, \nu \times v o l, i_{*} \sum_{\substack{x \in \sigma_{n},\left|p_{n} x_{n}\right|<s}} \delta_{x_{n}}^{2 d} \times \eta_{M}\left(Y_{x, n}\right)\right) \\
& =\left(M \times M,(p, p), v o l \times \delta_{p}, \nu \times v o l, i_{*} \sum_{\substack{x \in \sigma,\left|p_{n} x_{n}\right|<s}} \delta_{x_{n}}^{2 d} \times \eta_{M}\left(Y_{x, n}\right)\right)
\end{aligned}
$$

where the equality holds for sufficiently large $n$ as the is a one-to-one correspondence between $x_{n}$ 's and $x$ 's. This definition is by construction consistent with our previous definition 3.20 of $Y_{n}^{(s)}$.

It remains to check (3.21). We claim that this pointwise convergence holds via the $\iota_{n}$ 's. Note that a map from the equivalence class $Y_{n}^{(s)}(\omega)$ to $M$ is defined up to isometries of $(M, p, \sigma)$. Observe that

$$
\iota_{n *}^{4 d} i_{*} \sum_{\substack{x \in \sigma,\left|p_{n} x_{n}\right|<s}} \delta_{x_{n}}^{2 d} \times \eta_{M}\left(Y_{x, n}\right)=i_{*} \sum_{\substack{x \in \sigma,\left|p_{n} x_{n}\right|<s}} \delta_{\iota_{n} x_{n}}^{2 d} \times \eta_{M}\left(\iota_{n} Y_{x, n}\right)
$$

a.s. Moreover for large $n$ we have

$$
=i_{*} \sum_{\substack{x \in \sigma \\|p x|<s}} \delta_{X_{x, n}}^{2 d} \times \eta_{M}\left(X_{x, n}\right) .
$$

But this means that this converges for almost every $\omega \in \Omega$ to the strainer of $Y^{(s)}(\omega)$. This proves that $Y_{n}^{(s)}$ a.s. converges to $Y^{(s)}(\omega)$. Since $s$ was chosen arbitrarily and by our observation from (3.21), $X_{n}$ converges a.s. to $X$. Hence $m_{(M, p, \nu)}^{S t r}$ is continuous.

The Integration Lemma 1.7 gives the map Str. It remains to check the section property and unimodularity. 
Section property. $\quad$ Define the section map $\operatorname{Str}(S(\overline{\mathfrak{M}})) \rightarrow \operatorname{SP} \underline{\mathcal{M}}$

$$
\begin{aligned}
p^{S t r}:(M, d, p, \mu, \sigma, \vec{\nu}) & \\
& \mapsto\left(\operatorname{supp} \mu,\left.\frac{1}{2} d\right|_{(\operatorname{supp} \mu) \times(\operatorname{supp} \mu)} p, \mu \cap(\operatorname{supp} \mu), \sigma \cap(\operatorname{supp} \mu)\right) .
\end{aligned}
$$

Note that the domain of this map is complete since it is compact as the image of a compact set under continuous maps. By Lemma 3.24 it is sufficient to check that $\left(\mathcal{P} p^{S t r}\right) \circ \mu_{M}=\delta_{M}$ for every $M \in \mathrm{SP} \underline{\mathcal{M}}$. But this holds true since for any bounded continuous test function $\varphi: \operatorname{SP} \underline{\mathcal{M}} \rightarrow \mathbb{R}$ we have $\int \varphi \circ p^{S t r} \mathrm{~d} \mu_{M}=\varphi(M)$.

Unimodularity of $S t r . \quad$ Let $\varphi: \operatorname{StrPP} \mathcal{M} \rightarrow \mathbb{R}$ be an arbitrary bounded test function with bounded support. According to the discussion following Definition 2.2 it is to check that $\int \varphi \mathrm{d} m_{\mathrm{L}}(\operatorname{Str} P)=\int \varphi \mathrm{d} m_{\mathrm{R}}(\operatorname{Str} P)$ for any unimodular law $\bar{P}$ on $S(\overline{\mathfrak{M}})$. We calculate

$$
\begin{aligned}
& \int \varphi \mathrm{d} m_{\mathrm{L}}(\operatorname{Str} P) \\
&= \iint \varphi\left(M^{\prime}, p^{\prime}, q^{\prime}, \mu^{\prime}, \sigma, \vec{\nu}^{\prime}\right) \mathrm{d} m_{\mathrm{L}}\left(m_{(M, p, \sigma)}^{\operatorname{Str}}\right)\left(M^{\prime}, p^{\prime}, q^{\prime}, \mu^{\prime}, \sigma, \vec{\nu}^{\prime}\right) \\
& \mathrm{d} P(M, p, \sigma)
\end{aligned}
$$

by definition of Pmm-convergence and by continuity of $\varphi$ we have

$$
\begin{array}{r}
=\iiint \lim _{s \rightarrow \infty} \varphi\left(M^{2},(p, p),(q, q), \mu^{\prime}, \sigma \times \operatorname{vol}, \vec{\nu}^{\prime} \cap \mathrm{B}((p, p), s)^{d}\right) \\
\mathrm{d} \operatorname{vol}(q) \mathrm{d} \tilde{m}_{(M, p, \sigma)}^{\operatorname{Str}}\left(M^{\prime}, p^{\prime}, \mu^{\prime}, \sigma^{\prime}, \vec{\nu}^{\prime}\right) \mathrm{d} P(M, p, \sigma)
\end{array}
$$

By definition of the metric of doubly pointed spaces 1.25 the distance of an argument of $\varphi$ to the argument $\left(M^{2},(p, p),(p, q), \ldots\right)$ is bounded from below by $|p q|$. Since the support of $\varphi$ is bounded, the integrand is bounded by a constant depending only on $(M, p, \sigma)$. Hence the Dominated Convergence Theorem Dud02, Theorem 4.3.5] is applicable.

$$
\begin{array}{r}
=\int \lim _{s \rightarrow \infty} \iint \varphi\left(M^{2},(p, p),(q, q), \mu^{\prime}, \sigma \times v o l, \vec{\nu}^{\prime} \cap \mathrm{B}((p, p), s)^{d}\right) \\
\mathrm{d} \operatorname{vol}(q) \mathrm{d} \tilde{m}_{(M, p, \sigma)}^{\mathrm{Str}}\left(M^{\prime}, p^{\prime}, \mu^{\prime}, \sigma^{\prime}, \vec{\nu}^{\prime}\right) \mathrm{d} P(M, p, \sigma) \\
=\int \lim _{s \rightarrow \infty} \int \ldots \iint_{x \in \sigma \cap \mathrm{B}(p, s)} \int \varphi\left(M^{2},(p, p),(q, q), \mu^{\prime}, \sigma \times v o l, i_{*} \delta_{p^{\prime}}^{d} \times \vec{x}\right) \\
\mathrm{d} \operatorname{vol}(q) \underbrace{\ldots \mathrm{d} \bar{f}_{x} v o l^{2 d}(\vec{x}) \ldots}_{x \in \sigma \cap \mathrm{B}(p, s)} \mathrm{d} P(M, p, \sigma)
\end{array}
$$

by Tonelli-Fubini Theorem Dud02, Theorem 4.4.5]

$$
\begin{gathered}
=\int \lim _{s \rightarrow \infty} \iint_{x \in \sigma \cap \mathrm{B}(p, s)} \ldots \int_{x \in \sigma \cap \mathrm{B}(p, s)} \varphi(\underbrace{2},(p, p),(q, q), \mu^{\prime}, \sigma \times \operatorname{vol}, i_{*} \delta_{p^{\prime}}^{d} \times \vec{x}) \\
\ldots \mathrm{d} \bar{f}_{x} v o l^{2 d}(\vec{x}) \ldots \\
\mathrm{d} \operatorname{vol}(q) \mathrm{d} P(M, p, \sigma)
\end{gathered}
$$


reverting some steps from above we get

$$
\begin{array}{r}
=\iiint \varphi\left(M^{2},(p, p),(q, q), \mu^{\prime}, \sigma \times \operatorname{vol}, \vec{\nu}^{\prime}\right) \\
\mathrm{d} \tilde{m}_{(M, p, \sigma)}^{\operatorname{Str}}\left(\vec{\nu}^{\prime}\right) \mathrm{d} \operatorname{vol}(q) \mathrm{d} P(M, p, \sigma)
\end{array}
$$

due to invariance under representatives of equivalence classes in SP $\underline{\mathcal{M}}(3.17$

$$
\begin{array}{r}
=\iiint \varphi\left(M^{2},(p, p),(q, q), \mu^{\prime}, \sigma \times v o l, \vec{\nu}^{\prime}\right) \\
\mathrm{d} \tilde{m}_{(M, p, \sigma)}^{\mathrm{Str}}\left(\vec{\nu}^{\prime}\right) \mathrm{d}\left(m_{\mathrm{L}} P\right)(M, p, q, \sigma)
\end{array}
$$

apply unimodularity of $P$

$$
\begin{aligned}
& =\iiint \varphi\left(M^{2},(p, p),(q, q), \mu^{\prime}, \sigma \times \operatorname{vol}, \vec{\nu}^{\prime}\right) \\
& \mathrm{d} \tilde{m}_{(M, p, \sigma)}^{\mathrm{Str}}\left(\vec{\nu}^{\prime}\right) \mathrm{d}\left(m_{\mathrm{R}} P\right)(M, p, q, \sigma) \\
& =\iiint \varphi\left(M^{2},(p, p),(q, q), \mu^{\prime}, \sigma \times \operatorname{vol}, \vec{\nu}^{\prime}\right) \mathrm{d} \tilde{m}_{(M, p, \sigma)}^{\mathrm{Str}}\left(\vec{\nu}^{\prime}\right) \\
& \operatorname{d} \operatorname{vol}(p) \mathrm{d} P(M, q, \sigma)
\end{aligned}
$$

use invariance under change of base point 3.18

$$
\begin{aligned}
& =\iiint \varphi\left(M^{2},(p, p),(q, q), \mu^{\prime}, \sigma \times \operatorname{vol}, \vec{\nu}^{\prime}\right) \mathrm{d} \tilde{m}_{(M, q, \sigma)}^{\mathrm{Str}}\left(\vec{\nu}^{\prime}\right) \\
& \mathrm{d} \operatorname{vol}(p) \mathrm{d} P(M, q, \sigma) \\
& =\iiint \varphi\left(M^{2},(p, p),(q, q), \mu^{\prime}, \sigma \times \operatorname{vol}, \vec{\nu}^{\prime}\right) \mathrm{d} \operatorname{vol}(p) \mathrm{d} \tilde{m}_{(M, q, \sigma)}^{\mathrm{Str}}\left(\vec{\nu}^{\prime}\right) \\
& \mathrm{d} P(M, q, \sigma) \\
& =\iint \varphi\left(M^{\prime}, p^{\prime}, q^{\prime}, \mu^{\prime}, \sigma, \vec{\nu}^{\prime}\right) \mathrm{d} m_{\mathrm{R}}\left(m_{(M, q, \sigma)}^{\mathrm{Str}}\right)\left(M^{\prime}, p^{\prime}, q^{\prime}, \mu^{\prime}, \sigma, \vec{\nu}^{\prime}\right) \\
& \mathrm{d} P(M, q, \sigma) \\
& =\int \varphi \mathrm{d} m_{\mathrm{R}}(\operatorname{Str} P)
\end{aligned}
$$

\subsubsection{Third step: random curvature tensor}

We will use Theorem 3.15 on automorphism rigidity and 3.21 on chart selection to construct a random curvature tensor of a space endowed with a strainer, i.e. a map

$$
m^{R}: \operatorname{StrP} \underline{\mathcal{M}} \rightarrow \mathcal{P} \mathrm{RP} \underline{\mathcal{M}}
$$

Third Step of Proof of Lemma 2.23. For Euclidean balls $B \supset B_{2}, B^{\prime} \supset B_{3}$, a metric $g \in \mathrm{C}^{\alpha}\left(B, \mathrm{Sym}^{2}\right)$ and $\alpha^{\prime} \in(0,1)$ define the condition $\left(\mathrm{I}_{B, B^{\prime}}^{\alpha^{\prime}}\right)$

$g$ is the $\mathrm{C}^{\alpha}$-limit of pullbacks $\varphi_{n}^{*} g^{n}$ some smooth Riemannian metrics $g^{n}$ on $B_{4}$ under isometries $\varphi_{n}: B^{\prime} \rightarrow B_{4}$ such that

$$
B \subset \mathrm{B}_{\varphi_{n}^{*} g^{n}}\left[0, e^{\Theta+1} \text { radius } B\right] \subset B^{\prime \circ} \text { and } \operatorname{inj}_{0} g^{n} \geq 5 e^{\Theta+1} \quad\left(\mathrm{I}_{B, B^{\prime}}^{\alpha^{\prime}}\right)
$$

where $B^{\prime \circ}:=\mathrm{B}_{\text {Eucl. }}\left(0\right.$, radius $\left.B^{\prime}\right)$ is the interior of $B^{\prime}$. Define the $\mathrm{C}^{\beta^{\prime}}$-closed set

$$
\mathcal{B}^{\prime}:=\left\{g \in \mathrm{C}^{\beta^{\prime \prime}}\left(B_{3}^{\prime}, \mathrm{Sym}^{2}\right) \mid \begin{array}{r}
\|g\|_{\mathrm{C}^{\beta^{\prime \prime}}} \leq \varrho^{-\alpha} \Xi, 2 r \vec{e} \text { is an } \varepsilon \text {-strainer of } 0, \\
\left(\mathrm{I}_{B, B^{\prime}}^{\beta^{\prime \prime}}\right), \text { and }\left.g\right|_{B_{0}} \text { is harmonic }
\end{array}\right\}
$$


This set is compact by Arzelà-Ascoli. The points $2 r \vec{e}$ form an $\varepsilon$ strainer of 0 for every metric in $g \in \mathcal{B}^{\prime}$ since being an $\varepsilon$-strainer is a closed condition. Moreover the condition $\left(\mathrm{I}_{B, B^{\prime}}^{\beta^{\prime}}\right)$ holds for every metric in $\mathcal{B}^{\prime}$ by a diagonal argument. Also the restriction $\left.g\right|_{B_{0}}$ of any $g \in \mathcal{B}^{\prime}$ is harmonic since harmonicity as defined in $\S 1.3 .3$ by 1.22 is a closed condition.

By Theorem 1.21, part (ii) there is a bound $C=C\left(d, \beta^{\prime}, \varrho, \Theta, r\right)$ on the $\mathrm{C}^{1, \alpha}$-norm of the restriction $\left.f\right|_{B_{2}^{\prime}}$ for any differentiable map $f: e B_{2}^{\prime} \rightarrow \mathbb{R}^{d}$ such that $f\left(e B_{2}^{\prime}\right) \subset B_{3}^{\prime}$ and $g^{\prime}=f^{*} g$ for metrics $g^{\prime}$ on $e B_{2}^{\prime}$ and $g$ on $B_{3}^{\prime}$ with $\|g\|_{\mathrm{C}^{\beta^{\prime}}},\left\|g^{\prime}\right\|_{\mathrm{C}^{\beta^{\prime}}} \leq \varrho^{-\alpha} \Xi$. Apply Theorem 3.21 for $\alpha=\beta^{\prime}, \beta=\beta, B_{2}^{\prime}=B_{2}^{\prime}$, $B_{3}^{\prime}=B_{3}^{\prime}$, and $\mathcal{F}=\mathrm{B}_{\mathrm{C}^{\beta^{\prime}}\left(B_{2}^{\prime}, \mathbb{R}^{d}\right)}(i d, C)$ to every $g \in \mathcal{B}^{\prime}$ obtaining open sets $\mathcal{G}_{g}$ and maps

$$
P=P_{g}: \mathcal{G} \longrightarrow i d+\mathrm{C}^{1, \beta}\left(B_{2}^{\prime}, \mathbb{R}^{d}\right)_{2 r \vec{e}}
$$

Since $P$ is continuous, we can assume by further shrinking $\mathcal{G}_{g}$ that the Lipschitz constant of

$$
P_{g}\left(g^{\prime}\right):\left(B_{2}^{\prime}, d_{\text {Eucl. }}\right) \rightarrow\left(B_{3}^{\prime}, d_{\text {Eucl. }}\right)
$$

is at most $e^{1 / 2}$ and $\left\|P_{g}\left(g^{\prime}\right)-i d\right\|_{\mathrm{C}^{1, \beta}}<e^{-\Theta}$ for every $g^{\prime} \in \mathcal{G}_{g}$. By compactness we can find a finite set

$$
\left\{g^{1}, \ldots, g^{k}\right\} \subset \mathcal{B}^{\prime} \text { and } \operatorname{radii} r_{1}, \ldots, r_{k}
$$

such that $\mathrm{B}_{\mathrm{C}^{\beta^{\prime}}\left(B_{3}^{\prime}, \mathrm{Sym}^{2}\right)}\left(g^{1}, r_{1}\right), \ldots, \mathrm{B}_{\mathrm{C}^{\beta^{\prime}}\left(B_{3}^{\prime}, \mathrm{Sym}^{2}\right)}\left(g^{k}, r_{k}\right)$ cover $\mathcal{B}^{\prime}$. Let $\lambda_{1}, \ldots$, $\lambda_{k}$ be a corresponding partition of unity.

For each space $(N, q, \mu, \sigma, \vec{\nu}) \in \operatorname{StrP} \mathcal{M}$ fix a model $(M, p)$, thereby we mean an actually pointed Riemannian manifold such that $\left(M \times M,(p, p), \Delta_{*} v o l\right)$ is contained in the equivalence class $(N, q, \mu)$. By abuse of notation we write $\sigma=\sigma \cap(\operatorname{supp} \mu) \subset M$. For each $x \in \sigma$ we have a corresponding $\varepsilon$-strainer $\vec{z}$ given by $\vec{\nu}$ (see definition from previous subsection). As $M \in \mathrm{P} \underline{\mathcal{M}}$, it is a limit of smooth manifolds $M_{n}$ with $\left\|M_{n}\right\|_{\mathrm{C}^{\alpha}}^{\varrho}$ har $\leq \Theta$. Therefore, by the Fundamental Theorem of Convergence Theory, Theorem 1.24 we know that $\left\|M_{n}\right\|_{\mathrm{C}^{\beta^{\prime \prime}}}^{\varrho \text { har }} \leq \Theta$. By Proposition 3.13 we can find at least one chart $\varphi:\left(B_{3}^{\prime}, 0\right) \rightarrow(M, x)$ such that $\varphi^{*} g \in \mathcal{B}^{\prime}$. Moreover $g \in \mathrm{B}_{\mathrm{C}^{\beta^{\prime}}\left(B_{3}^{\prime}, \mathrm{Sym}^{2}\right)}\left(g^{j}, r_{j}\right)$ for at least one $j=1, \ldots, k$. We call a $\varphi$ subject to both conditions (i.e. $\varphi^{*} g \in \mathcal{B}^{\prime}$ and $g \in \mathrm{B}_{\mathrm{C}^{\beta^{\prime}}\left(B_{3}^{\prime}, \mathrm{Sym}^{2}\right)}\left(g^{j}, r_{j}\right)$ ) admissible. Due to these observations we can define a variation of $\lambda_{1}, \ldots, \lambda_{k}$ :

$$
\begin{aligned}
\tilde{\lambda}_{i}(M, x, \vec{z}) & :=\sup \left\{\lambda_{i}\left(\varphi^{*} g\right) \mid \varphi:\left(B_{3}^{\prime}, 0\right) \rightarrow(M, x) \text { is admissible }\right\} \cup\{0\}, \\
\lambda_{i}(M, x, \vec{z}) & :=\frac{1}{\sum_{j=1}^{k} \tilde{\lambda}_{j}(M, x, \vec{z})} \tilde{\lambda}_{i}(M, x, \vec{z}),
\end{aligned}
$$

for $i=1, \ldots, k$ and an $\varepsilon$-strainer $\vec{z}$. Note that the denominator in this definition is at least 1 . If the space $M$ is clear from the context, we write

$$
\lambda_{i, x}:=\lambda_{i}(M, x, \vec{z})
$$

Set $s=\frac{1}{2}$ radius $B_{0}$. Let $b: \mathbb{R} \rightarrow[0,1]$ be a smooth bump with $b(0)=1$ and $\operatorname{supp} b=[-1,1]$ and $\left.b\right|_{\mathrm{B}[0,1 / 2]}=1$. For a pointed chart $\varphi: \frac{1}{2} B_{0} \rightarrow(M, x)$ define

$$
b_{\varphi}: M \rightarrow[0, \infty), \quad b_{\varphi}(y):= \begin{cases}0 & \text { if } y \notin \varphi\left(\frac{1}{2} B_{0}\right) \\ b\left(\left|0 \varphi^{-1}(y)\right|_{\text {Eucl. }}^{2} / s^{2}\right) & \text { else. }\end{cases}
$$


For a locally finite system of pointed charts $\left\{\varphi_{i}\right\}_{i \in I}$ define

$$
b_{i}\left(\left\{\varphi_{i}\right\}_{i \in I}\right)(y):=\frac{1}{\sum_{j \in I} b_{\varphi_{j}}(y)} b_{\varphi_{i}}(y)
$$

for $y \in M$. A C $\mathrm{C}^{\alpha}$-bound on the $b_{i}\left(\left\{\varphi_{i}\right\}_{i \in I}\right)(y)$ 's will be established in the paragraph on continuity below.

Recall from $\S 1.3 .6$ that $\mathrm{C}^{\beta^{\prime}}\left(M, \mathrm{~T}^{3,1} M\right)$ consists of $(3,1)$-tensors that are $\mathrm{C}^{\beta^{\prime}}$ function on some charts in the differentiable atlas of $M$. Further, define a topology on $\mathrm{C}^{\beta^{\prime}}\left(M, \mathrm{~T}^{3,1} M\right)$ by saying that a sequence of sections $s_{n}$ converges to $s$ if and only if for every open bounded set $\Omega \subset M$ there is a cover of $\Omega$ by charts with harmonic $\mathrm{C}^{\alpha}$-norm on the scale of $\varrho$ at most $\Theta$ such that on these charts the sections converge as $\mathrm{C}^{\beta^{\prime}}$-functions. By definition the embedding

$$
\iota_{(M, p)}: U \rightarrow \operatorname{RP} \stackrel{\mathcal{M}}{\longrightarrow}, s \mapsto(M, p, s), \quad U \subset \mathrm{C}^{\beta^{\prime}}\left(M, \mathrm{~T}^{3,1} M\right)
$$

is continuous for a subset $U$ such that there is a uniform bound on the $\mathrm{C}^{\beta^{\prime}}$-norm for any $s \in U$ on some charts with harmonic $\mathrm{C}^{\alpha}$-norm on the scale of $\varrho$ at most $\Theta$.

After these preparations we can define the integration map. Let $B_{R} \subset M$ be the ball of radius $r$ around $p$ of a representative $(M, p)$ of an equivalence class $N=(N, q, \sigma, \vec{\nu}) \in \mathrm{RP} \mathcal{M}$. Recall Definition 2.14 of $R\left(\left\{\varphi_{i}\right\},\left\{\lambda_{i}\right\}\right)$ and the bump function $b_{x, r}$ from (1.10). Observe that the set $\sigma \cap B_{R}$ is finite. Let $i_{x} \in\{1, \ldots, k\}$ be a choice of indices for each $x \in \sigma \cap B_{R}$. The product $\prod_{i} \lambda_{i_{x}, x}$ vanishes if there is no admissible pointed chart with $\varphi_{x}^{*} g \in \mathrm{B}_{\mathrm{C}^{\beta^{\prime}}\left(B_{3}^{\prime}, \mathrm{Sym}^{2}\right)}\left(g^{i}, r_{i}\right)$ for all $x \in \sigma \cap B_{R}$. Define

$$
\begin{aligned}
\varphi_{x}^{i_{x}} & :=\varphi_{x} \circ P_{g^{i_{x}}}\left(\varphi_{x}^{*} g\right) \\
\tilde{m}_{N, M, B_{R}}^{R} & :=\underbrace{\sum_{1}^{k} \lambda_{\ldots} \ldots \sum_{i_{x}=1}^{k} \lambda_{i_{x}, x} \ldots \delta_{b_{p, R} \cdot R}\left(\left\{\left.\varphi_{x}^{i_{x}}\right|_{\frac{1}{2} B_{0}}\right\}_{x},\left\{b_{x}\left(\left\{\left.\varphi_{x}^{i_{x}}\right|_{\frac{1}{2} B_{0}}\right\}_{x}\right)\right\}_{x}\right)}_{x \in \sigma \cap B_{R}} \\
& =\underbrace{\ldots \sum_{i_{x}=1}^{k} \cdots \prod_{x \in \sigma \cap B_{R}} \lambda_{i_{x}, x} \delta}_{x \in \sigma \cap B_{R}}{ }_{b_{p, R} \cdot R}\left(\left\{\left.\varphi_{x}^{i_{x}}\right|_{\frac{1}{2} B_{0}}\right\}_{x},\left\{b_{x}\left(\left\{\left.\varphi_{x}^{i_{x}}\right|_{\frac{1}{2} B_{0}}\right\}_{x}\right)\right\}_{x}\right)
\end{aligned}
$$

where always $x \in \sigma \cap B_{R}$, and $\varphi_{x}$ denotes an admissible pointed chart. Note, that being a convex combination of Dirac measures the sum $\tilde{m}_{N, M, B_{R}}^{R}$ is a probability measure on $\mathrm{C}^{0}\left(M, \mathrm{~T}^{3,1} M\right)$. We have to show that this expression is well-defined, i.e.

(i) does not depend on the choice of an admissible charts $\varphi_{x}$;

(ii) actually defines a tensor, i.e. the transition functions are $\mathrm{C}^{2, \beta}$-bounded and partition of unity is $\mathrm{C}^{1, \beta}$-bounded.

For the first point take two admissible charts $\varphi_{x}$ and $\varphi_{x}^{\prime}$ and some index $i$ such that $\varphi_{x}^{*} g \in \mathrm{B}_{\mathrm{C}^{\beta^{\prime}}\left(B_{3}^{\prime}, \operatorname{Sym}^{2}\right)}\left(g^{i}, r_{i}\right)$. We have to show that $\varphi^{i}=\varphi^{\prime i}$ Set $g . .:=\varphi^{*} g$ and $g_{.}^{\prime}:=\varphi^{\prime *} g$. Since $\left.g_{.}\right|_{B_{2}^{\prime}}=\left(\varphi \circ \varphi^{\prime-1}\right)^{*} g_{.}^{\prime}$, Theorem 3.21 implies that the 
metrics $g_{.}^{i}:=P_{g^{i}}\left(g_{\ldots} .\left.\right|_{B_{3}^{\prime}}\right)^{*} g_{\text {. . }}$ and $P_{g^{i}}\left(g^{\prime} .\left.\right|_{B_{3}^{\prime}}\right)^{*} g^{\prime}$. coincide. In other words, we have an isometry $\left(B_{2},\left.g_{.}^{i}\right|_{B_{2}}\right) \rightarrow\left(B_{2}^{\prime}, g_{.}^{i}\right)$ given by $f:=\left(\varphi^{\prime} \circ P_{g^{i}}\left(g^{\prime} .\right)^{-1} \circ(\varphi \circ\right.$ $\left.P_{g^{i}}(g .).\right)$. We check that our rigidity theorem, Theorem 3.15, is applicable to $\alpha=\beta^{\prime}, B_{2}^{\prime}=B_{2}, B_{3}^{\prime}=B_{3}$, and $f=f$ : The chart $\varphi$ can be obtained by Proposition 3.13 from a chart $\psi:(\mathrm{B}(0, \varrho), 0) \rightarrow(M, x)$ with $\|\psi\|_{\mathrm{C}^{\beta^{\prime \prime}}} \leq \Theta$ with $\varphi=\psi \circ \tilde{\varphi}$. Due to 3.22 we have

$$
\begin{aligned}
& P_{g^{i}}\left(B_{2}\right) \\
& \subset e^{\frac{1}{2}} B_{2}=\mathrm{B}_{\text {Eucl. }}\left[0,4 e^{\Theta+\frac{3}{2}} r\right] \subset \mathrm{B}_{g .}\left[0,4 e^{2 \Theta+\frac{3}{2}} r\right] \subset \mathrm{B}_{\tilde{\varphi}^{*} g_{n}}\left[0,4 e^{2 \Theta+\frac{3}{2}} r\right] \\
& \subset \mathrm{B}_{\text {Eucl. }}\left(0,4 e^{3(\Theta+1)} r\right)=B_{3}^{\circ}
\end{aligned}
$$

for sufficiently large $n$. As the approximations $g_{n}$ we chosen from manifolds $\left(M_{n}, x_{n}\right) \in \mathcal{M}$, we have $\operatorname{inj}_{0} g^{n} \geq 5 e^{\Theta+1}$. Hence condition $\left(\mathrm{I}_{B_{2}, B_{3}}^{\beta^{\prime}}\right)$ holds. The map $f$ preserves the strainer as all maps it is composed of do. The remaining assumptions hold as well by construction. Hence we can apply Theorem 3.15 and have $f=i d$. Thus $\varphi^{\prime} \circ P_{g^{i}}\left(g_{.}^{\prime}=\varphi \circ P_{g^{i}}\left(g_{.}.\right)\right.$. This proves the independence of the choice of $\varphi$.

For the second point observe that the charts $\left.\varphi_{x}^{i_{x}}\right|_{\frac{1}{2} B_{0}}$ are actually harmonic, since they are shrunk to their harmonic part, and even have a harmonic buffer of Euclidean width $\frac{1}{2}$ (radius $B_{0}$ ) around them. Hence the transition functions between to such charts is actually bounded by $\tilde{C}=\tilde{C}(d, \varrho, \Theta, \beta, r)$ in virtue of Theorem 1.21. Note, that the denominator in $(3.24)$ is actually bounded

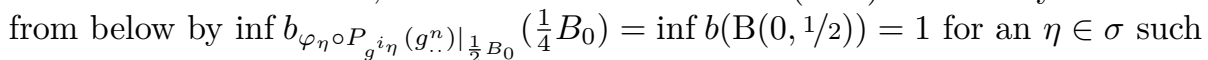
that $|\eta \xi|_{g}<\frac{1}{2} e^{-\Theta-1}=\frac{1}{2}$ (radius $B_{0}$ ) which exists because $\sigma$ is $\tilde{r}$-covering and $\tilde{r} \leq \frac{1}{4} e^{-2(\Theta+1)} r$. The partition of unity defined by 3.24 is bounded in the $\mathrm{C}^{1, \beta}$-norm since $P_{g^{i}}(g) \leq \Theta+1$ and a chart $\varphi$ is bounded by $\Xi$ in the $\mathrm{C}^{1, \beta^{\prime}}$-norm. Hence we see that there is even a uniform bound

$$
C=C(d, \varrho, \Theta, \beta, r)
$$

on the $\mathrm{C}^{\beta}$-norm of $R\left(\left\{\left.\varphi_{x}^{i_{x}}\right|_{\frac{1}{2} B_{0}}\right\}_{x}\left\{b_{x}\left(\left\{\left.\varphi_{x}^{i_{x}}\right|_{\frac{1}{2} B_{0}}\right\}_{x}\right)\right\}_{x}\right)$ restricted to $B_{R}$.

Next we extend $\tilde{m}_{N, M, B_{R}}^{R}$ to a random curvature tensor on the entire $M$. To this end define

$$
\tilde{m}_{N, M}^{R}:=\lim _{R \rightarrow \infty} \tilde{m}_{N, M, B_{R}}^{R}
$$

where convergence is a consequence of the Dominated Convergence Theorem: For $r=1,2, \ldots$, we describe each law $\tilde{m}_{N, M, B_{R}}^{R}$ by a random variable

$$
X_{R}:([0,1), \lambda) \rightarrow \mathrm{C}^{0}\left(M, \mathrm{~T}^{3,1} M\right)
$$

with Law $X_{R}=\tilde{m}_{N, M, B_{R}}^{R}$ and $\lambda$ the Lebesgue measure. Enumerate the $x$ 's in $\sigma$ by $1,2, \ldots$ such that if $n<m$ then $x_{m} \in \sigma \cap B_{R}$ implies $x_{n} \in \sigma \cap B_{R}$. For each $n=1, \ldots$ subdivide $[0,1)$ into $k^{n}$ (possibly empty) intervals of lengths given by $\lambda_{1}\left(M, x_{n}\right), \ldots, \lambda_{k}\left(M, x_{n}\right)$, i.e. for $n=1$ take

$$
\left[0, \lambda_{1}\left(M, x_{1}\right)\right), \ldots,\left[\lambda_{1}\left(M, x_{1}\right)+\ldots+\lambda_{k-1}\left(M, x_{1}\right), 1\right),
$$

for $n=2$ take

$$
\begin{array}{r}
{\left[0, \lambda_{1}\left(M, x_{1}\right) \cdot \lambda_{1}\left(M, x_{2}\right)\right), \ldots,\left[\lambda_{1}\left(M, x_{1}\right) \cdot \sum_{i=1}^{k-1} \lambda_{i}\left(M, x_{2}\right), \lambda_{1}\left(M, x_{1}\right)\right),} \\
\ldots,\left[\sum_{i=1}^{k-1} \lambda_{i}\left(M, x_{1}\right)+\lambda_{k}\left(M, x_{1}\right)\left(\lambda_{1}\left(M, x_{2}\right)+\ldots+\lambda_{k-1}\left(M, x_{2}\right)\right), 1\right),
\end{array}
$$


and so forth. Let $I(n)$ denote the set of such intervalls. We can index the intervalls by a tupel $\left(i_{1}, \ldots, i_{n}\right) \in\{1, \ldots, k\}^{n}$. For each $r=1, \ldots$ let $n_{r}:=\# \sigma \cap B_{R}$. We define $X_{R}$ as the step function that is constant on each intervall in $I\left(n_{r}\right)$ and assigns to an interval with index $\left(i_{1}, \ldots, i_{n}\right)$ with value $b_{p, r} R\left(\left\{\left.\varphi_{x_{m}}^{i_{m}}\right|_{\frac{1}{2} B_{0}}\right\}_{x_{m}}\left\{b_{x_{m}}\left(\left\{\left.\varphi_{x_{m}}^{i_{m}}\right|_{\frac{1}{2} B_{0}}\right\}_{m}\right)\right\}_{m}\right)$ where $m$ ranges over $1, \ldots, n_{r}$. Observe that Law $X_{n}=\tilde{m}_{N, M, B_{R}}^{R}$. These random variables converge pointwise since the restriction $\left.X_{n}\right|_{\Omega}$ to a bounded open domain become constant in $n$ for sufficiently large $n$. From this follows convergence in law as for any bounded continuous test function $f: \mathrm{C}^{0}\left(M, \mathrm{~T}^{3,1} M\right) \rightarrow \mathbb{R}$ we have $\lim _{R \rightarrow \infty} \int f \mathrm{~d} \tilde{m}_{N, M, B_{R}}^{R}=$ $\lim _{R \rightarrow \infty} \int f \circ X_{R} \mathrm{~d} \lambda=\int \lim _{R \rightarrow \infty} f \circ X_{R} \mathrm{~d} \lambda$ by the Dominated Convergence Theorem Dud02, Theorem 4.3.5]. In particulart, this theorem implis that the pointwise limit

$$
X(\omega)=\lim _{R \rightarrow \infty} X_{R}(\omega)
$$

is a measurable function.

Further note that the bound on the chart norm (3.27) now holds on the entire space $M$. We can therefore use the map $\iota_{(M, p)}$ defined in 3.25 to get the integration map

$$
m_{(N, q, \mu, \sigma, \vec{\nu})}^{R}:=\iota_{(M, p) *} \tilde{m}_{N, M}^{R}
$$

where $(M, p)$ is a model for $(N, q, \mu, \sigma, \vec{\nu})$ as considered above. As the construction of $\tilde{m}_{N, M, B_{R}}^{R}$ is preserved under isomorphisms and in the construction of $\tilde{m}_{N, M}^{R}$ the enumeration of the points in $\sigma$ was irrelevant, $m_{(N, q, \mu, \sigma, \vec{\nu})}^{R}$ is independent of the choice of a model space.

Continuity of $m^{R}$. Take a converging sequence $m_{\left(N_{n}, q_{n}, \mu_{n}, \sigma_{n}, \vec{\nu}_{n}\right)}^{R}$. Take some models $\left(M_{n}, p_{n}\right)$ for these spaces. To show convergence is sufficient to show that the random variables

$$
\iota_{\left(M_{n}, p_{n}\right)} \circ X^{n},
$$

where the $X^{n}$ 's are the random variables from 3.28, pointwise converge to $\iota_{(M, p)} \circ X$. To this end first observe that for pointwise convergence it is sufficient to look at a ball $B_{R}$ of arbitrary size and find comparison maps $\iota_{n}: M_{n} \rightarrow M$ such that on $B_{R}$ we have $\mathrm{C}^{\beta}$-convergence $\iota_{n *} X^{n} \rightarrow X$. Next observe that for this purpose it is actually fine to look only at the $(r+1)$-approximations $X_{r+1}^{n}$, $X_{r+1}$ of $X^{n}, X$. Finally, observe that for sufficiently large $n$ there are for each $x \in B_{R} \cap \sigma$ unique $x_{n}$ 's in $\sigma_{n}$ such that $\iota_{n}\left(x_{n}\right) \rightarrow x$ for $n \rightarrow \infty$. We are at liberty to arrange the numbering of the points in $\sigma_{n}$ for the subdivision on the interval $[0,1)$ in such a way that it is consistent with the numbering of $\sigma$.

Further observe that $\lambda_{i}\left(M_{n}, x_{n}\right)$ converges to $\lambda_{i}(M, x)$ provided that $\iota_{n}\left(x_{n}\right)$ converge $x$ for $n \rightarrow \infty$ since the denominator in 3.23 is bounded by 1 . As the $\lambda_{i}\left(M_{n}, x_{n}\right)$ converge, the probability that $\omega \in[0,1)$ is in the same interval of the subdivision indexed by $\left(i_{1}, \ldots, i_{k}\right) \in\{1, \ldots, k\}^{n}$ converges to one. Hence we can assume that $X_{n}(\omega)$ and $X(\omega)$ choose the same indices $i_{1}, \ldots, i_{k}$.

Further take for any point $y \in M$ a neighborhood $U$ that is so small that it is contained in the image of $\varphi \circ P_{g^{i}}\left(\varphi^{*} g\right)$ for some chart $\varphi: \mathrm{B}(0, \varrho) \rightarrow M$ with $\|\varphi\|_{\mathrm{C}^{\beta^{\prime}}} \leq \Theta$. On $\mathrm{B}(0, \varrho)$ the metric $\varphi^{*} g$ is approximated by $g_{. .}^{n}:=\varphi^{*} \iota_{n *} g_{n}$. By making this chart a bit smaller and restricting to sufficiently large $n$, we can even assume that $U$ is contained in $\psi \circ\left(P_{g^{i}}\left(\psi^{*} \iota_{n *} g_{n}\right)\right)\left(\frac{1}{2} B_{0}\right)$ for each pointed chart $\psi: \mathrm{B}(0, \varrho) \rightarrow(M, x)$ for each $x \in \sigma$ such that $y \in \psi \circ\left(P_{g^{i}}\left(\psi^{*} \iota_{n *} g_{n}\right)\right)\left(\frac{1}{2} B_{0}\right)$. 
On the chart $\varphi_{U}: V \rightarrow U \subset M$ the curvature tensor of the piecewise Euclidean connection

$$
R\left(\left\{\left.\varphi_{x} \circ P_{g^{i x}}\left(g_{. .}^{n}\right)\right|_{\frac{1}{2} B_{0}}\right\}_{x \in \sigma}\left\{b_{x}\left(\left\{\left.\varphi_{x} \circ P_{g^{i x}}\left(g_{. .}^{n}\right)\right|_{\frac{1}{2} B_{0}}\right\}_{x}\right)\right\}_{x \in \sigma}\right)
$$

is given in coordinates, according to its definition 2.8 , by

$$
\begin{aligned}
\varphi_{U} R_{\lambda \mu \nu}^{k}=\sum_{\xi \in \sigma} b_{\xi}\left(\left\{\left.\varphi_{\eta} \circ P_{g^{i} \eta}\left(g_{. .}^{n}\right)\right|_{\frac{1}{2} B_{0}}\right\}_{\eta \in \sigma}\right) \sum_{l}\left(\left(x_{k, l}^{n, \xi} \circ y^{n, \xi}\right)_{, \mu} y_{l, \nu \lambda}^{n, \xi}\right)_{[\mu \nu]} \\
+\left(b_{\xi}\left(\left\{\left.\varphi_{\eta} \circ P_{g^{i \eta}}\left(g_{. .}^{n}\right)\right|_{\frac{1}{2} B_{0}}\right\}_{\eta \in \sigma}\right)_{, \mu} \Gamma_{\nu \lambda}^{k}\right)_{[\mu \nu]} \\
+\sum_{\kappa}\left({ }^{\varphi_{U}} \Gamma_{\mu \kappa}^{k} \varphi_{U} \Gamma_{\nu \lambda}^{\kappa}\right)_{[\mu \nu]}
\end{aligned}
$$

where

- the $y^{n, \xi}$ 's are of the form $P_{g^{i x}}\left(g_{. .}^{n}\right)^{-1} \circ \varphi^{-1}$ for a chart $\varphi=\varphi_{n}$ independent of $n$ with $\|\varphi\|_{\mathrm{C}^{\beta}} \leq \Xi$;

- the $x^{n, \xi}$ are of the form $P_{g^{i x}}\left(g_{. .}^{n}\right)^{-1} \circ \varphi^{-1} \circ \varphi^{\prime} \circ P_{g^{i x}}\left(g_{. .}^{\prime n}\right)$ with $g_{. .}^{\prime n}:=\varphi^{\prime *} \iota_{n *} g_{n}$ for charts $\varphi=\varphi_{\xi}, \varphi^{\prime}=\varphi_{\xi}^{\prime}$ independent of $n$ with $\|\varphi\|_{\mathrm{C}^{\beta}},\left\|\varphi^{\prime}\right\|_{\mathrm{C}^{\beta}} \leq \Xi$; and

- ${ }^{\varphi_{U}} \Gamma_{\mu \nu}^{k}=\sum_{\xi \in \sigma} b_{\xi}(\ldots) \sum_{l}\left(x_{k, l}^{n, \xi} \circ y^{n, \xi}\right)\left(y_{l, \mu \nu}^{n, \xi}\right)$.

Due to the product estimate from Corollary 1.11 and the composition estimate from Lemma 1.13 for convergence of (3.30), we have to check convergence of

- the $b_{\xi}$ 's in $\mathrm{C}^{1, \beta}$ : Note, that the denominator in 3.24 is actually bounded from below by inf $b_{\left.\varphi_{\eta} \circ P_{g^{i \eta}}\left(g^{n} .\right)\right|_{\frac{1}{2} B_{0}}}\left(\frac{1}{4} B_{0}\right)=\inf b(\mathrm{~B}(0,1 / 2))=1$ for an $\eta \in \sigma$ such that $|\eta \xi|_{g}<\frac{1}{2} e^{-\Theta-1}=\frac{1}{2}$ (radius $B_{0}$ ) which exists because $\sigma$ is $\tilde{r}$-covering and $\tilde{r} \leq \frac{1}{4} e^{-2(\Theta+1)} r$. Due to this fact, Corollary 1.11 and Lemmas 1.12 and 1.13 and since the Euclidean distance function is smooth, the partition of unity $b_{x}\left(\left\{\left.\varphi_{x}^{i_{x}}\right|_{\frac{1}{2} B_{0}}\right\}_{x}\right)$ for $x \in \sigma_{n}$ converges in $\mathrm{C}^{1, \beta}$;

- the $y^{n, \xi}=P_{g^{i_{x}}}\left(g_{. .}^{n}\right)^{-1} \circ \varphi^{-1}$ 's in $\mathrm{C}^{2, \beta}$;

- the $x^{n, \xi}=P_{g^{i_{x}}}\left(g_{. .}^{n}\right)^{-1} \circ \varphi^{-1} \circ \varphi^{\prime} \circ P_{g^{i_{x}}}\left(g_{. .}^{\prime n}\right)$ in $\mathrm{C}^{2, \beta}$.

Note, that by continuity of $P_{g^{i x}}$ the $P_{g^{i x}}\left(g_{. .}^{n}\right)$ 's already converge in $\mathrm{C}^{1, \beta}$. The inverses of the $P_{g^{i_{x}}}\left(g_{.}^{n}\right)^{\text {'s }}$ are of class $\mathrm{C}^{1, \ddot{\beta}}$ by Theorem 1.21 part (i) By Corollary 1.18 and the assumption $\left\|P_{g}\left(g^{\prime}\right)-i d\right\|_{\mathrm{C}^{1, \beta}}<e^{-\Theta}$, also the inverses of the $P_{q^{i x}}\left(g^{n}\right)$ 's converge if the domain is restricted by a factor $e^{-\Theta}$. Finally, Lemma 1.23 improves the convergence regularity to $\mathrm{C}^{2, \beta}$, if we restrict to the harmonic part $\frac{1}{2} B_{0}$ of the charts. Therefore, the composition estimate from Lemma 1.13 implies convergence of the $y^{n, \xi}$ 's and the $x^{n, \xi}$ 's as $n \rightarrow \infty$. This proves convergence of (3.30) as $n \rightarrow \infty$. Hence we have convergence of $\iota_{n *} X_{n}(\omega)$ to $X(\omega)$ on $U$. Since we can cover $B_{R}$ by such $U$ 's we have $\iota_{n *} X_{n}(\omega)$ to $X(\omega)$ for almost every $\omega$. This proves continuity.

The map $\tilde{R}$ is now defined as in the previous steps by the Integration Lemma 1.7 
Unimodularity of $R$. Take some unimodular law $P$ on $\operatorname{StrP} \mathcal{M}$. Next let $\varphi$ a continuous bounded test function with bounded support on $\mathrm{RPP} \stackrel{\mathcal{M}}{\rightarrow}$. For the law $\tilde{R}(P)$ observe

$$
\begin{array}{rl}
\int f & \mathrm{~d} m_{\mathrm{L}}(\tilde{R}(P)) \\
& =\iint f \mathrm{~d} m_{\mathrm{L}}\left(m_{(N, q, \mu, \sigma, \vec{\nu})}^{R}\right) \mathrm{d} P(N, q, \mu, \sigma, \vec{\nu}) \\
& =\iiint f\left(M, p, p^{\prime}, s\right) \mathrm{d} \operatorname{vol}_{M}\left(p^{\prime}\right) \mathrm{d} m_{(N, q, \mu, \sigma, \vec{\nu})}^{R}(M, p, s) \mathrm{d} P(N, q, \mu, \sigma, \vec{\nu}) \\
& =\iiint f\left(M, p, p^{\prime}, s\right) \mathrm{d} \operatorname{vol}_{M}\left(p^{\prime}\right) \mathrm{d} \tilde{m}_{(N, M)}^{R}(s) \mathrm{d} P(N, q, \mu, \sigma, \vec{\nu}) \\
& =\iint_{[0,1)} \int f\left(M, p, p^{\prime}, X(\omega)\right) \mathrm{d} v o l_{M} \mathrm{~d} \lambda(\omega) \mathrm{d} P(N, q, \mu, \sigma, \vec{\nu}) \\
& =\iint_{[0,1)} \int f\left(M, p, p^{\prime}, X(\omega)\right) \mathrm{d} \lambda(\omega) \mathrm{d} v_{M} l_{M}\left(p^{\prime}\right) \mathrm{d} P(N, q, \mu, \sigma, \vec{\nu})
\end{array}
$$

since Law $X$ is invariant under the choice of representative of $N \in \operatorname{StrP} \stackrel{\mathcal{M}}{\rightarrow}$

$$
=\iint_{[0,1)} \int f\left(M, p, q^{\prime}, X(\omega)\right) \mathrm{d} \lambda(\omega) \mathrm{d}\left(m_{\mathrm{L}} P\right)\left(N, q, q^{\prime}, \mu, \sigma, \vec{\nu}\right)
$$

by unimodularity of $P$

$$
\begin{aligned}
& =\iint_{[0,1)} \int f\left(M, p, p^{\prime}, X(\omega)\right) \mathrm{d} \lambda(\omega) \mathrm{d}\left(m_{R} P\right)\left(N, q, q^{\prime}, \mu, \sigma, \vec{\nu}\right) \\
& =\iint_{[0,1)} \int f\left(M, p, p^{\prime}, X(\omega)\right) \mathrm{d} \lambda(\omega) \mathrm{d} v o l_{M}(p) \mathrm{d} P\left(N, q^{\prime}, \mu, \sigma, \vec{\nu}\right) \\
& =\iint_{[0,1)} \int f\left(M, p, p^{\prime}, X(\omega)\right) \mathrm{d} \operatorname{vol}_{M}(p) \mathrm{d} \lambda(\omega) \mathrm{d} P\left(N, q^{\prime}, \mu, \sigma, \vec{\nu}\right) \\
& =\iiint \int_{[0,1)} \int f\left(M, p, p^{\prime}, X(\omega)\right) \mathrm{d} v o l_{M}(p) \mathrm{d} \tilde{m}_{(N, M)}^{R}(s) \mathrm{d} P\left(N, q^{\prime}, \mu, \sigma, \vec{\nu}\right) \\
& =\iint f \mathrm{~d} m_{\mathrm{R}}\left(m_{(N, q, \mu, \sigma, \vec{\nu})}^{R}\right) \mathrm{d} P(N, q, \mu, \sigma, \vec{\nu}) \\
& =\int f \mathrm{~d} m_{\mathrm{R}}(\tilde{R}(P)) .
\end{aligned}
$$

As we proved that each map $S, S t r$, and $\tilde{R}$ preserves unimodularity, we know that also $R=\tilde{R} \circ S t r \circ S$ does.

Section property. Regarding the section property we can not check it for $\tilde{R}$ because this maps forgets the strainer. Instead we prove it for $D$. The

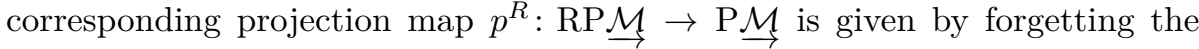
strainer. Let $\tilde{p}: \operatorname{Str}(S(\overline{\mathcal{M}})) \rightarrow \mathrm{P} \stackrel{\mathcal{M}}{\rightarrow}$ be the map that forgets the strainer, i.e. that 
maps $(M, p, \mu, \sigma, \vec{\nu})$ to $\left(\operatorname{supp} \mu, p,\left.\mu\right|_{\operatorname{supp} \mu}\right)$. The maps are part of the diagram

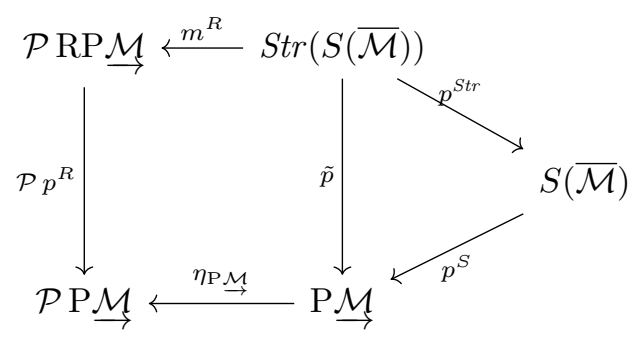

where $p^{S}$ and $p^{S t r}$ are the projection maps from the preceding two part of the proof. The triangle in this diagram commutes by definition. The square in this diagram commutes because for any space $N=(N, q, \mu, \sigma, \vec{\nu}) \in \operatorname{StrP} \mathcal{M}$ for any measurable function $f$ on $\mathrm{P} \underset{\mathcal{M}}{\longrightarrow}$ we have

$$
\begin{aligned}
\int f \mathrm{~d}\left(\mathcal{P} p^{R}\right)\left(m_{N}^{R}\right) & =\int f \circ p^{R} \mathrm{~d} m_{N}^{R}=\int f(M, p) \mathrm{d} m_{N}^{R}(M, p, s) \\
& =f\left(\operatorname{supp} \mu, p,\left.\mu\right|_{\operatorname{supp} \mu}\right) .
\end{aligned}
$$

From this the section property of $R$ follows from the abstract nonsense of the Giry monad and our proofs of the section properties of $S$ and Str:

Using properties of the Giry monad from Lemma 1.8 , the map $D$ can be expressed as

$$
D=\mu_{\mathrm{RP}} \underset{\mathcal{M}}{\rightarrow} \circ\left(\mathcal{P} m^{R}\right) \circ \mu_{\mathrm{StrP}} \underset{\mathcal{M}}{ } \circ\left(\mathcal{P} m^{S t r}\right) \circ \mu_{\mathrm{SP}} \underset{\mathcal{M}}{\rightarrow} \circ\left(\mathcal{P} m^{S}\right)
$$

use that $\mu$ is a counit to $\eta$

$$
=\mu_{\mathrm{RP}} \underset{\mathcal{M}}{\rightarrow} \circ \eta_{\mathcal{P} \mathrm{RP}} \stackrel{\mathcal{M}}{\rightarrow} \circ \mu_{\mathrm{RP}} \underset{\mathcal{M}}{ } \circ\left(\mathcal{P} m^{R}\right) \circ \mu_{\mathrm{StrP}} \stackrel{\mathcal{M}}{\rightarrow} \circ\left(\mathcal{P} m^{S t r}\right) \circ \mu_{\mathrm{SP}} \underset{\mathcal{M}}{ } \circ\left(\mathcal{P} m^{S}\right)
$$

omit indices of $\eta, \mu$ and use naturality of $\eta, \mu$

$$
=\mu \circ \mathcal{P}\left(\mu \circ\left(\mathcal{P} m^{R}\right) \circ \mu \circ\left(\mathcal{P} m^{S t r}\right) \circ \mu \circ\left(\mathcal{P} m^{S}\right)\right) \circ(\mathcal{P} \eta)
$$

use functoriality

$$
=\mu \circ \mathcal{P}\left(\mu \circ\left(\mathcal{P} m^{R}\right) \circ \mu \circ\left(\mathcal{P} m^{S t r}\right) \circ \mu \circ\left(\mathcal{P} m^{S}\right) \circ \eta\right)
$$

use naturality of $\eta, \mu$

$$
\begin{aligned}
& =\mu \circ \mathcal{P}\left(\mu \circ\left(\mathcal{P} m^{R}\right) \circ \mu \circ\left(\mathcal{P} m^{S t r}\right) \circ m^{S}\right) \\
& =\mu \circ \mathcal{P}\left(\mu \circ \mu \circ(\mathcal{P} \eta) \circ\left(\mathcal{P} m^{R}\right) \circ \mu \circ\left(\mathcal{P} m^{S t r}\right) \circ m^{S}\right) \\
& =\mu \circ \mathcal{P}\left(\mu \circ \mu \circ \mathcal{P}\left(\left(\mathcal{P} m^{R}\right) \circ \mu \circ\left(\mathcal{P} m^{S t r}\right)\right) \circ(\mathcal{P} \eta) \circ m^{S}\right) \\
& =\mu \circ \mathcal{P}\left(\mu \circ \mu \circ \mathcal{P}\left(\left(\mathcal{P} m^{R}\right) \circ \mu \circ\left(\mathcal{P} m^{S t r}\right) \circ \eta\right) \circ m^{S}\right) \\
& =\mu \circ \mathcal{P}\left(\mu \circ \mu \circ \mathcal{P}\left(\left(\mathcal{P} m^{R}\right) \circ m^{S t r}\right) \circ m^{S}\right) \\
& =\mu \circ \mathcal{P}\left(\mu \circ \mathcal{P}(\mu) \circ\left(\mathcal{P} \mathcal{P} m^{R}\right) \circ\left(\mathcal{P} m^{S t r}\right) \circ m^{S}\right)
\end{aligned}
$$

This is to say that the map $D$ is given by integration of the map $m^{R}:=$ $\mu_{\mathrm{RP} \underset{\mathcal{M}}{\rightarrow}} \circ \mathcal{P}\left(\mu_{\mathrm{RP}} \underset{\mathcal{M}}{\rightarrow}\right) \circ\left(\mathcal{P} \mathcal{P} m^{R}\right) \circ\left(\mathcal{P} m^{S t r}\right) \circ m^{S}$. Hence we can use Lemma 3.24 To this end observe that

$$
(\mathcal{P} p) \circ m^{R}=\mu_{\mathrm{RP}} \underset{\mathcal{M}}{\rightarrow} \mathcal{P}\left(\mu_{\mathrm{RP}} \underset{\mathcal{M}}{\rightarrow}\right) \circ(\mathcal{P} \mathcal{P} \mathcal{P} p) \circ\left(\mathcal{P} \mathcal{P} m^{R}\right) \circ\left(\mathcal{P} m^{S t r}\right) \circ m^{S}
$$


using commutativity of the square in 3.31

$$
\begin{aligned}
& =\mu_{\mathrm{RP}} \underset{\rightarrow}{\mathcal{M}} \circ \mathcal{P}\left(\mu_{\mathrm{RP}} \underset{\mathcal{M}}{\rightarrow}\right) \circ \mathcal{P} \mathcal{P}\left(\eta_{\mathrm{P}} \underset{\mathcal{M}}{ } \circ \tilde{p}\right) \circ\left(\mathcal{P} m^{S t r}\right) \circ m^{S} \\
& =\mu_{\mathrm{RP} \underset{\mathcal{M}}{\rightarrow}} \circ \mathcal{P} \mathcal{P}\left(p^{S} \circ p^{S t r}\right) \circ\left(\mathcal{P} m^{S t r}\right) \circ m^{S}
\end{aligned}
$$

use the equalities $\left(\mathcal{P} p^{S}\right) \circ m^{S}=\eta$ and $\left(\mathcal{P} p^{S t r}\right) \circ m^{S t r}=\eta$ proved in the two preceding parts of the proof

$$
\begin{aligned}
& =\mu_{\mathrm{P} \mathcal{M}} \circ \mathcal{P} \mathcal{P}\left(p^{S}\right) \circ \mathcal{P}\left(\eta_{\mathrm{SP}} \underline{\mathcal{M}}\right) \circ m^{S} \\
& =\mu_{\mathrm{P} \underline{\mathcal{M}}} \circ \mathcal{P}\left(\eta_{\mathrm{P}} \underline{\mathcal{M}} \circ p^{S}\right) \circ m^{S} \\
& =\mathcal{P}\left(p^{S}\right) \circ m^{S} \\
& =\eta_{\mathrm{P}} \underline{M} \text {. }
\end{aligned}
$$

Lemma 3.24 now implies the section property of $R$. 


\section{Appendix A}

\section{Metrization and metrizability theorems}

\section{A.1 Spaces of pointed metric spaces}

Define for two pointed spaces $(M, p)$ and $(N, q)$

$$
\begin{aligned}
\mathrm{d}_{\mathrm{PGH}}(M, N) & :=\sum_{r=1}^{\infty} r^{-2} \wedge \mathrm{d}_{\mathrm{PGH}}^{\prime}(\mathrm{B}[p, r], \mathrm{B}[q, r]) \\
\mathrm{d}_{\mathrm{PGH}}^{\prime}(M, N) & :=\inf _{\iota, \eta} \mathrm{d}_{\mathrm{H}}(\iota M, \eta N)+|\iota(p) \eta(q)|
\end{aligned}
$$

where the infimum is taken over all embeddings $\iota: \mathrm{B}[p, r] \rightarrow L$ and $\eta: \mathrm{B}[q, r] \rightarrow L$ to some compact metric space $L=(L, \mid$. .|).

Theorem 1.3. On PM a metric is given by $\mathrm{d}_{\mathrm{PGH}}$, that is complete, separable, and induces the same notion of convergence as defined by 1.2 and 1.3 .

Proof. The proof proceeds in five steps.

$\mathrm{d}_{\mathrm{PGH}}$ is a pseudometric, i.e. a metric that does not neceissarily distinguishes points. The function $\mathrm{d}_{\mathrm{PGH}}^{\prime}$ is obviously symmetric, so is $\mathrm{d}_{\mathrm{PGH}}$. To see the triangle inequality it is sufficient to check it for the function $\mathrm{d}_{\mathrm{PGH}}^{\prime}$. For any $\varepsilon>0$ consider compact spaces $M, M^{\prime}, N$ and a zig-zag of embeddings

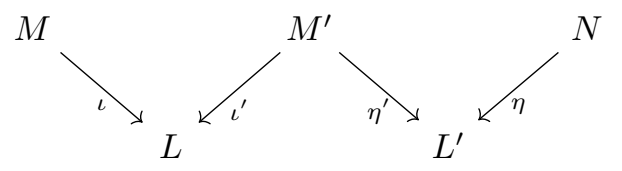

such that

$$
\begin{array}{ccc}
\mathrm{d}_{\mathrm{H}}\left(\iota M, \iota^{\prime} M^{\prime}\right)+\left|\iota(p) \iota^{\prime}\left(p^{\prime}\right)\right| & \text { and } & \mathrm{d}_{\mathrm{H}}\left(\eta^{\prime} M^{\prime}, \eta N\right)+\left|\eta^{\prime}\left(p^{\prime}\right) \eta(q)\right| \\
\leq \mathrm{d}_{\mathrm{PGH}}^{\prime}\left(M, M^{\prime}\right)+\varepsilon & & \leq \mathrm{d}_{\mathrm{PGH}}^{\prime}\left(M^{\prime}, N\right)+\varepsilon
\end{array}
$$


Next we form the pushout $L \sqcup_{M^{\prime}} L^{\prime}$ along the maps $\iota^{\prime}$ and $\eta^{\prime}$, i.e. the set theoretic pushout with the metric

$$
d(x, y)= \begin{cases}d_{L}(x, y) & \text { if } x, y \in L \\ d_{L^{\prime}}(x, y) & \text { if } x, y \in L^{\prime} \\ \inf \left\{d_{L}(x, z)+d_{L^{\prime}}(z, y) \mid z \in L \sqcup_{M^{\prime}} L^{\prime}\right\} & \text { else, }\end{cases}
$$

that is indeed well-defined and a metric by a quick check or by [BBI01, § 3.1]. We denote the inclusion maps by $i_{L}: L \rightarrow L \sqcup_{M^{\prime}} L^{\prime}$ and $i_{L^{\prime}}: L^{\prime} \rightarrow L \sqcup_{M^{\prime}} L^{\prime}$ and write $M^{\prime}=i_{L}\left(\iota^{\prime} M^{\prime}\right)=i_{L^{\prime}}\left(\eta^{\prime} M^{\prime}\right) \subset L \sqcup_{M^{\prime}} L^{\prime}$ as well as $p^{\prime}=i_{L}\left(\iota^{\prime}\left(p^{\prime}\right)\right)=$ $i_{L^{\prime}}\left(\eta^{\prime}\left(p^{\prime}\right)\right) \in L \sqcup_{M^{\prime}} L^{\prime}$. On closed subsets of the metric space $L \sqcup_{M^{\prime}} L^{\prime}$ the triangle inequality holds for Hausdorff distance $\mathrm{d}_{\mathrm{H}}$. Hence,

$$
\begin{aligned}
\mathrm{d}_{\mathrm{PGH}}^{\prime}(M, N) \leq & \mathrm{d}_{\mathrm{H}}\left(i_{L}(\iota M), i_{L^{\prime}}(\eta N)\right)+\left|i_{L}(\iota(p)) i_{L^{\prime}}(\eta(q))\right| \\
\leq & \mathrm{d}_{\mathrm{H}}\left(i_{L}(\iota M), M^{\prime}\right)+\mathrm{d}_{\mathrm{H}}\left(M^{\prime}, i_{L^{\prime}}(\eta N)\right) \\
& \quad+\left|i_{L}(\iota(p)) p^{\prime}\right|+\left|p^{\prime} i_{L^{\prime}}(\eta(q))\right| \\
= & \mathrm{d}_{\mathrm{H}}\left(\iota M, \iota^{\prime} M^{\prime}\right)+\left|\iota(p) \iota^{\prime}\left(p^{\prime}\right)\right| \\
\quad & \quad \mathrm{d}_{\mathrm{H}}\left(\eta^{\prime} M^{\prime}, \eta N\right)+\left|\eta^{\prime}\left(p^{\prime}\right) \eta(q)\right| \\
\leq & \mathrm{d}_{\mathrm{PGH}}^{\prime}\left(M, M^{\prime}\right)+\mathrm{d}_{\mathrm{PGH}}^{\prime}\left(M^{\prime}, N\right)+2 \varepsilon .
\end{aligned}
$$

As $\varepsilon>0$ was chosen arbitrarily, the triangle inequality follows.

$\mathrm{d}_{\mathrm{PGH}}$ and PGH-convergence coincide. First assume that a sequence $M_{n}=$ $\left(M_{n}, d_{n}, p_{n}\right) \mathrm{PGH}$-converges to a space $M$ by some $\varepsilon$-isometries $\iota_{n}: \mathrm{B}\left[p_{n}, r\right] \rightarrow M$ subject to 1.3. As we have restricted ourselves to length spaces, we can modify each $\iota_{n}$ such that $\iota_{n}\left(\mathrm{~B}\left[p_{n}, r\right]\right) \subset \mathrm{B}[p, r]$ by going back along paths: For each $x \in \iota_{n}\left(\mathrm{~B}\left[p_{n}, r\right]\right) \backslash \mathrm{B}[p, r]$ we know that $|p x| \leq r+\varepsilon$ and that there is a geodesic $\gamma_{x}$ joining $p$ and $x$. The function $\tilde{\iota}_{n}$

$$
\tilde{\iota}_{n}(x):= \begin{cases}\gamma_{x}\left(\operatorname{length}\left(\gamma_{x}\right)-\varepsilon\right) & \text { if } x \in \iota_{n}\left(\mathrm{~B}\left[p_{n}, r\right]\right) \backslash \mathrm{B}[p, r] \\ x & \text { else. }\end{cases}
$$

in a $2 \varepsilon$-isometry. Rename $\tilde{\iota}_{n}$ by $\iota_{n}$ and $2 \varepsilon$ by $\varepsilon$.

On the disjoint union $M_{n} \sqcup M$ define the symmetric function

$$
d(x, y):= \begin{cases}\inf _{\xi \in M_{n}}|x \xi|+\left|y \iota_{n}(\xi)\right|+\varepsilon & \text { if } x \in M_{n} \text { and } y \in M \\ d_{n}(x, y) & \text { if } x, y \in M_{n} \\ d(x, y) & \text { if } x, y \in M .\end{cases}
$$

This as a metric as it distinguishes points and the triangle inequality holds as

$$
\begin{aligned}
d(x, y) & \leq \inf _{\xi, \zeta} d_{n}(x, \xi)+d_{n}(\xi, \eta)+d_{n}(\eta, y) \\
& \leq \inf _{\xi, \zeta} d_{n}(x, \xi)+d\left(\iota_{n}(\xi), \iota_{n}(\eta)\right)+\varepsilon+d_{n}(\eta, y) \\
& \leq d(x, z)+d(z, y)
\end{aligned}
$$

for $x, y \in M_{n}$ and $z \in M$,

$$
\begin{aligned}
d(x, y) & \leq \inf _{\xi, \zeta} d\left(x, \iota_{n}(\xi)\right)+d\left(\iota_{n}(\xi), \iota_{n}(\eta)\right)+d\left(\iota_{n}(\eta), y\right) \\
& \leq \inf _{\xi, \zeta} d\left(x, \iota_{n}(\xi)\right)+d(\xi, \eta)+\varepsilon+d\left(\iota_{n}(\eta), y\right) \\
& \leq d(x, z)+d(z, y)
\end{aligned}
$$


for $x, y \in M$ and $z \in M_{n}$, and

$$
\begin{aligned}
d(x, y) & \leq \inf _{\xi} d_{n}(x, \xi)+d\left(\iota_{n}(\xi), z\right)+d\left(\iota_{n}(\eta), y\right) \\
& \leq \inf _{\xi, \zeta} d\left(x, \iota_{n}(\xi)\right)+d(\xi, \eta)+\varepsilon+d\left(\iota_{n}(\eta), y\right) \\
& \leq d(x, z)+d(z, y)
\end{aligned}
$$

for $x, z \in M_{n}$ and $y \in M$. Note that we still have $d\left(p_{n}, p\right) \leq \varepsilon$. Hence $\mathrm{d}_{\mathrm{PGH}}^{\prime}\left(\mathrm{B}\left[p_{n}, r\right], \mathrm{B}[p, r]\right) \leq 2 \varepsilon$ for sufficiently large $n$. The existence of this metric implies $\mathrm{d}_{\mathrm{H}}\left(\iota \mathrm{B}\left[p_{n}, r\right], \eta \mathrm{B}[p, r]\right) \leq \varepsilon$ for sufficiently large $n$. As $\varepsilon>0$ can be chosen arbitrarily small, $M_{n}$ converges to $M$ with respect to $\mathrm{d}_{\mathrm{PGH}}$.

In the other direction we observe that for any $r>0$ convergence with respect to the pseudometric $(M, N) \mapsto \mathrm{d}_{\mathrm{PGH}}^{\prime}\left(\mathrm{B}\left[p_{n}, r\right], \mathrm{B}[p, r]\right)$ implies the existence of a suitable $\iota_{n}$ 's by taking close-by points, i.e. modifying a $\tilde{\iota}_{n}: \mathrm{B}\left[p_{n}, r\right] \rightarrow L$ given from the condition $\mathrm{d}_{\mathrm{PGH}}^{\prime}\left(\mathrm{B}\left[p_{n}, r\right], \mathrm{B}[p, r]\right)<\varepsilon$ by mapping $x \in M_{x}$ to some point $\iota_{n}(x) \in M \subset L$ with $\left|\iota(n) \tilde{\iota}_{n}(x)\right|<\varepsilon$.

The pseudometric $d_{\mathrm{PGH}}$ distinguishes points, i.e. it is a metric. Theorem 8.1.7 from [BBI01] states that pointed GH-convergence distinguishes points, i.e. proper metric spaces. Hence $\mathrm{d}_{\mathrm{PGH}}$ does.

Separability. Note that as stated in BBI01, Proposition 7.5.5] every compact length space can be GH-approximated by finite graphs, i.e. length spaces constructed from intervals of different lengths glued together at their endpoints. Hence any such space can be approximated by finite graphs in $\mathrm{P} \mathcal{M}$ with rational edge lengths. Moreover any proper metric space can be approximated by a compact space, i.e. taking a sufficiently large ball around the base point. Hence any space in PM can be PGH-approximated by pointed finite graphs with rational edge lengths. But there are only countably many such graphs.

Completeness. Take a $\mathrm{d}_{\mathrm{PGH}_{\mathrm{H}}}$-Cauchy-converging sequence $M_{n}$. Given $\varepsilon>0$ we can find a sufficiently large $n$ such that $\mathrm{d}_{\mathrm{GH}}\left(\mathrm{B}\left[p_{n}, r\right], \mathrm{B}\left[p_{k}, r\right]\right) \leq \varepsilon / 2$ for all $k \geq n$. Moreover there are $N$ points in the compact set $\mathrm{B}\left[p_{n}, r\right]$ that are an $\varepsilon / 2$-net (i.e. $\varepsilon / 2$-balls around these point cover $\mathrm{B}\left[p_{n}, r\right]$ ). Hence for any $\mathrm{B}\left[p_{k}, r\right]$ there is a $\varepsilon$-net consisting of $N=N(r, \varepsilon)$ points. Now [BBI01, Theorem 8.1.10] implies that the $M_{n}$ 's subconverge to some pointed metric space $M$. As any metric space is dense in its completion, we may assume without loss of generality that $M$ is complete. The space $M$ inherits from the spaces $M_{n}$ the properties of being a length space BBI01, Theorem 8.1.9] and of being proper BBI01, Exercise 8.1.8].

\section{A.2 Space of pointed mm-spaces}

To define a suitable metric on $\mathrm{PM}^{[k]}$, the space of (isomorphism classes) of mm-spaces with $k$ measures, first define the bump function $b_{x, r}$ by

$$
b_{x, r}(y)= \begin{cases}1 & \text { if }|x y| \leq r-1 \\ r-|x y| & \text { if } r-1 \leq|x y| \leq r \\ 0 & \text { if }|x y| \geq r\end{cases}
$$


We say that a sequence $\left(M_{n}\right)_{n} \subset \mathrm{PM}$ does Pmm-converge to $M$ if for all $r>0$ and $\varepsilon>0$ there are for sufficiently large $n$ measurable maps

$$
\iota_{n}: \mathrm{B}\left[p_{n}, r\right] \rightarrow M
$$

such that 1.3 holds and $\iota_{n *}\left(b_{p_{n}, r}(.) \operatorname{vol}_{n}\right)$ weak $\#$ converges to $b_{p, r} v o l$.

A further generalization are mm-spaces with $k$-measures $\left(M, d, v^{2} l_{1}, \ldots\right.$, $v l_{k}$ ) for some $k=1,2, \ldots$. The definition of convergence of mm-spaces is to be read mutatis mutandis, i.e. weak $\#$ convergence of $\iota_{n *}\left(b_{p_{n}, r}(). v l_{i n}\right)$ to $b_{p, r}(.) \operatorname{vol}_{i}$ for all $i=1, \ldots, k$ is required. Let $\mathrm{PM}^{[k]}$ denote the space of (equivalence classes of) pointed proper length spaces with $k$ measures.

On the space $\mathrm{PM}[k]$ we introduce the following metrization:

$$
\begin{aligned}
& d_{\mathrm{PM}}\left(M, M^{\prime}\right):=\sum_{r=1}^{\infty} r^{-2} \wedge d_{\mathrm{PM}}^{\prime}\left(\begin{array}{c}
\left(\mathrm{B}[p, r], b_{p, r} v_{0} l_{1}, \ldots, b_{p, r} v_{0} l_{k}, p\right), \\
\left(\mathrm{B}\left[p^{\prime}, r\right], b_{p^{\prime}, r} v^{\prime} l_{1}^{\prime}, \ldots, b_{p^{\prime}, r} v o l_{k}^{\prime}, p^{\prime}\right)
\end{array}\right)
\end{aligned}
$$

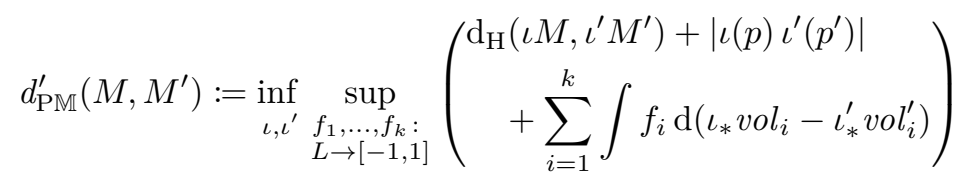

where the supremum is taken over all embeddings $\iota: M \rightarrow L$ and $\iota^{\prime}: M^{\prime} \rightarrow L$ to some compact metric space $L=(L,||$.$) , like in (1.7 \mathrm{~b})$; and the supremum is taken over all Lipschitz functions $f: L \rightarrow[-1,1]$ with Lipschitz constant not greater than 1.

This metrization is based on the Kantorovich-Rubinstein dual metric

$$
W(P, Q)=\sup _{f} \int f \mathrm{~d}(P-Q)
$$

where the infimum is taken over all Lipschitz functions $f: M \rightarrow[-1,1]$ with Lipschitz constant not greater than 1 (consider also Remark 1.5). This expression comes with the advantage that the triangle inequality is obvious as

$$
\begin{aligned}
\sup _{f} \int f \mathrm{~d}\left(P-P^{\prime}\right) & =\sup _{f} \int f \mathrm{~d}(P-Q)+\int f \mathrm{~d}\left(Q-P^{\prime}\right) \\
& \leq \sup _{f} \int f \mathrm{~d}(P-Q)+\sup _{g} \int g \mathrm{~d}\left(Q-P^{\prime}\right)
\end{aligned}
$$

for laws $P, P^{\prime}, Q$ on $M$.

Lemma A.1. Given a map $f:(M, p) \rightarrow(N, q)$ from a pointed compact metric space to a pointed metric space and $\varepsilon, r>0$ such that

$$
f(p)=q, \quad \operatorname{distor}(f) \leq \varepsilon, \quad \text { and } \quad \mathrm{B}[q, r-\varepsilon] \subset(f(M))^{\varepsilon)} .
$$

Then there is a measurable map $g$ with the same properties for $\varepsilon$ replaced by $3 \varepsilon$. Moreover $|g(x) f(x)| \leq 2 \varepsilon$ for all $x \in M$.

Proof. Take a finite ordered $\varepsilon$-cover of $\mathrm{B}[p, r]$, i.e. a system of balls $\mathrm{B}\left(x_{1}, \varepsilon\right), \ldots$, $\mathrm{B}\left(x_{l}, \varepsilon\right)$. Without loss of generality we may assume $p=x_{1}$ For each $x \in M$ there is a $\varepsilon$-close point of lowest index in the $\varepsilon$-covering. Define a map $g$ sending each point $x \in M$ to this closest point. 
The map $g$ is measurable, as every preimage of a measurable subset of $N$ is a finite union of the sets

$$
\begin{aligned}
\mathrm{B}\left(x_{1}, \varepsilon\right), \mathrm{B}\left(x_{2}, \varepsilon\right) \backslash \mathrm{B}\left(x_{1}, \varepsilon\right), \mathrm{B}\left(x_{3}, \varepsilon\right) \backslash\left(\mathrm{B}\left(x_{1}, \varepsilon\right) \cup \mathrm{B}\left(x_{2}, \varepsilon\right)\right), \ldots \\
\ldots, \mathrm{B}\left(x_{k}, \varepsilon\right) \backslash\left(\mathrm{B}\left(x_{1}, \varepsilon, \cup\right) \ldots \cup \mathrm{B}\left(x_{k-1} \varepsilon,\right)\right)
\end{aligned}
$$

By construction $g(p)=q$. Moreover for every $x, y \in M$ we have $|x y| \leq$ $\left|x x_{i}\right|+\left|x_{i} x_{j}\right|+\left|x_{j} y\right| \leq \varepsilon+\left(\left|f\left(x_{i}\right) f\left(x_{j}\right)\right|+\varepsilon\right)+\varepsilon=3 \varepsilon+\left|g\left(x_{i}\right) f\left(x_{j}\right)\right|$ for some indices $i, j \in\{1, \ldots, k\}$. For the same $x, y, i, j$ we also have $\left|g\left(x_{i}\right) f\left(x_{j}\right)\right| \leq$ $\left|x_{i} x_{j}\right|+\varepsilon \leq|x y|+2 \varepsilon+\varepsilon$. Hence we have distor $(g) \leq 3 \varepsilon$. For the last property take some point $y \in \mathrm{B}[q, r-\varepsilon]$. There is some point $x \in M$ such that $|f(x) y|<\varepsilon$. Let $i$ be the index such that $x_{i}$ is the point $\varepsilon$-close to $x$ of lowest index. Thus $\left|g\left(x_{i}\right) y\right| \leq\left|g\left(x_{i}\right) f(x)\right|+|f(x) y|=\left|f\left(x_{i}\right) f(x)\right|+|f(x) y| \leq\left|x_{i} x\right|+\varepsilon+\varepsilon \leq 3 \varepsilon$. The property $|g(x) f(x)| \leq\left|g(x) f\left(x_{i}\right)\right|+\left|f\left(x_{i}\right) f(x)\right|=0+\left|x_{i} x\right|+\varepsilon \leq 2 \varepsilon$ for a suitable index $i$.

Theorem 1.6. On $\mathrm{PM} \mathbb{M}^{[k]}$ a metric is given by $d_{\mathrm{PM}[k]}$, that is complete, separable, and induces the same notion of convergence as defined by (1.11).

Proof. The proof consists of four steps.

Metric. First, we check that $d_{\mathrm{PM}}$ is a metric: symmetry is obvious. For identity of indiscernibles first realize that $d_{\mathrm{PM}}\left(M, M^{\prime}\right)$ implies $\mathrm{d}_{\mathrm{PGH}}\left(M, M^{\prime}\right)=0$, i.e. $M$ and $M^{\prime}$ are isometric. Equality of measures is seen from the fact that Lipschitz functions distinguish signed measures on compact spaces (by Riesz representation theorem Dud02, Theorem 7.4.1] continuous functions do the same and Lipschitz functions on a compact space are dense among the latter Dud02, Theorem 11.2.4] with respect to the uniform norm).

For the last metric axiom, the triangle inequality, consider proper length mm-spaces $M, N$, and $M^{\prime}$. It is sufficient to show that the triangle inequality holds for $d_{\mathrm{PM}}^{\prime}\left(M, M^{\prime}\right)$ under the assumption that the radii of $M, N$, and $M^{\prime}$ are bounded by some $r>0$. In the following estimates every [-1.1]-valued function is assumed to be Lipschitz with Lipschitz constant at most 1.

$$
\begin{aligned}
& d_{\mathrm{PM}}^{\prime}\left(M, M^{\prime}\right) \\
& \quad \leq \inf _{\substack{\iota, \iota^{\prime} \\
(\iota M) \cup\left(\iota^{\prime} M^{\prime}\right) \rightarrow[-1,1]}} \sup _{\substack{f_{1}, \ldots, f_{k}: \\
\left(\iota c^{\prime}\right.}}\left(\mathrm{d}_{\mathrm{H}}\left(\iota M, \iota^{\prime} M^{\prime}\right)+\sum_{i=1}^{k} \int f_{i} \mathrm{~d}\left(\iota_{*} v^{\prime} l_{i}-\iota_{*}^{\prime} v^{\prime} l_{i}^{\prime}\right)\right)
\end{aligned}
$$

for embeddings $\iota: M \rightarrow L$ and $\iota^{\prime}: M^{\prime} \rightarrow L$. Assume additionally that there is an embedding $\eta: N \rightarrow L$.

$$
\leq \inf _{\substack{\iota, \eta, \iota^{\prime} \\(\iota M) \cup\left(\iota^{\prime} M^{\prime}\right) \rightarrow[-1,1]}} \sup _{\substack{f_{1}, \ldots, f_{k}: \\(\iota)}}\left(\mathrm{d}_{\mathrm{H}}\left(\iota M, \iota^{\prime} M^{\prime}\right)+\sum_{i=1}^{k} \int f_{i} \mathrm{~d}\left(\iota_{*} v o l_{i}-\iota_{*}^{\prime} v o l_{i}^{\prime}\right)\right)
$$

by the observation that any Lipschitz function extends from a closed subspace to the whole space preserving the Lipschitz constant [Bjö69, Proposition 1] we have

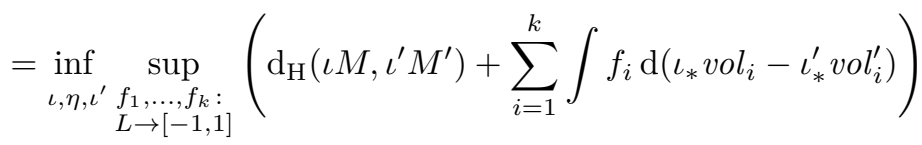


as the triangle inequality holds for $\mathrm{d}_{\mathrm{H}}$, points in $L$, and for measures in the way of $\mathrm{A} .2$, we further have

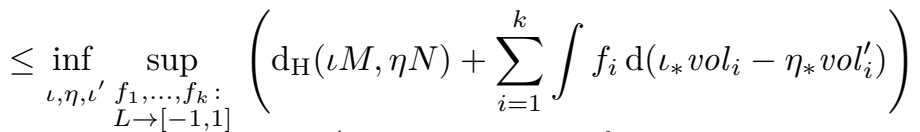

$$
\begin{aligned}
& +\left(\mathrm{d}_{\mathrm{H}}\left(\eta N, \iota^{\prime} M^{\prime}\right)+\sum_{i=1}^{k} \int f_{i} \mathrm{~d}\left(\eta_{*} \operatorname{vol}_{i}-\iota_{*}^{\prime} v^{\prime} l_{i}^{\prime}\right)\right) \\
& \leq \inf _{\iota, \eta, \iota^{\prime}}\left(\sup _{\substack{f_{1}, \ldots, f_{k}: \\
(\iota M) \cup(\eta N) \rightarrow[-1,1]}} \mathrm{d}_{\mathrm{H}}(\iota M, \eta N)+\sum_{i=1}^{k} \int f_{i} \mathrm{~d}\left(\iota_{*} v o l_{i}-\eta_{*} v o l_{i}^{\prime}\right)\right) \\
& +\left(\sup _{\substack{f_{1}, \ldots, f_{k}: \\
(\eta N) \cup\left(\iota^{\prime} M^{\prime}\right) \rightarrow[-1,1]}} \mathrm{d}_{\mathrm{H}}\left(\eta N, \iota^{\prime} M^{\prime}\right)+\sum_{i=1}^{k} \int f_{i} \mathrm{~d}\left(\eta_{*} v^{\prime} l_{i}-\iota_{*}^{\prime} v o l_{i}^{\prime}\right)\right) \\
& \leq\left(\inf _{\substack{\iota_{1}, \eta_{1} \\
\left(\iota_{1} M\right) \cup\left(\eta_{1} N\right) \rightarrow[-1,1]}} \sup _{\substack{\left.f_{1}, \ldots, f_{k}: \\
\eta_{1}\right) \rightarrow}} \mathrm{d}_{\mathrm{H}}\left(\iota_{1} M, \eta_{1} N\right)+\sum_{i=1}^{k} \int f_{i} \mathrm{~d}\left(\iota_{1 *} \operatorname{vol}_{i}-\eta_{1 *} v o l_{i}^{\prime}\right)\right) \\
& +\left(\inf _{\substack{\inf _{2}, \iota_{2}^{\prime}, f_{1}, \ldots, f_{k}: \\
\left(\eta_{2} N\right) \cup\left(\iota_{2}^{\prime} M^{\prime}\right) \rightarrow[-1,1]}} \mathrm{d}_{\mathrm{H}}\left(\eta_{2} N, \iota_{2}^{\prime} M^{\prime}\right)+\sum_{i=1}^{k} \int f_{i} \mathrm{~d}\left(\eta_{2 *} \text { vol }_{i}-\iota_{2 *}^{\prime} v o l_{i}^{\prime}\right)\right)
\end{aligned}
$$

for embeddings $\iota_{1}, \eta_{1}$ to some $L_{1}$ and $\eta_{2}, \iota_{2}$ to some $L_{2}$, where the inequality holds since $L_{1}$ and $L_{2}$ can be glued together along $N$ as metric spaces as in A.1.

$$
=d_{\mathrm{PM}}^{\prime}(M, N)+d_{\mathrm{PM}}^{\prime}\left(N, M^{\prime}\right) .
$$

The notion of convergence $(1.11)$ coincides with the notion induced by $\mathrm{PM}^{[k]}$. Take a sequence $M_{n}$ that converges in the sense of 1.11 . Fix any $r, \varepsilon>0$. By definition we get comparison maps $\iota_{n} M_{n} \rightarrow M$ for large $n$ as in 1.11. As in the proof of Theorem 1.3 we can construct from them metric spaces $M_{n} \sqcup M$ with embeddings $\tilde{\iota}_{n}: M_{n} \rightarrow M_{n} \sqcup M$ and $\tilde{\iota}: M \rightarrow M_{n} \sqcup M$ such that $\left|\tilde{\iota}\left(\iota_{n}(x)\right) \tilde{\iota}_{n}(x)\right| \leq \varepsilon$. For simplicity we will only calculate the case $k=1$. The following supremum is taken over all Lipschitz functions $f: M \rightarrow M_{n} \sqcup M \rightarrow$ $[-1,1]$ with Lipschitz constant not greater than one

$$
\begin{aligned}
\sup _{f}\left|\int f \mathrm{~d}\left(\tilde{\iota}_{n *} b_{p_{n}, r} v o l_{n}-\tilde{\iota}_{*} b_{p, r} v o l\right)\right| \\
=\sup _{f}\left|\int f \circ \tilde{\iota}_{n *} \mathrm{~d} b_{p_{n}, r} v o l_{n}-\int f \circ \tilde{\iota} \mathrm{d} b_{p, r} v o l\right| \\
=\sup _{f} \mid \int f \circ \tilde{\iota}_{n *} \mathrm{~d} b_{p_{n}, r} v o l_{n}-\int f \circ \tilde{\iota} \mathrm{d} b_{p, r} v o l \\
\quad-\int f \circ \tilde{\iota} \circ \iota_{n *} \mathrm{~d} b_{p_{n}, r} v o l_{n}+\int f \circ \tilde{\iota} \mathrm{d} \iota_{n *} b_{p_{n}, r} v o l_{n} \mid
\end{aligned}
$$




$$
\begin{aligned}
& =\sup _{f} \int\left|f \circ \tilde{\iota} \circ \iota_{n *}-f \circ \tilde{\iota}_{n *}\right| \mathrm{d} b_{p_{n}, r} v o l_{n} \\
& +\left|\int f \circ \tilde{\iota} \mathrm{d}\left(b_{p, r} \operatorname{vol}-\iota_{n *} b_{p_{n}, r} \operatorname{vol}_{n}\right)\right| \\
& \leq \varepsilon \int 1 \mathrm{~d}\left(b_{p, r} v o l-b_{p_{n}, r} v o l_{n}\right)+\sup _{f} \int f \circ \tilde{\iota} \mathrm{d}\left(b_{p, r} v o l-\iota_{n *} b_{p_{n}, r} v o l_{n}\right)
\end{aligned}
$$

the second summand only increases if we take the supremum over all continuous functions valued in $[-1,1]$. But then the second summand becomes, by a version of Riesz representation theorem Dud02, Theorem 7.4.1], equal to $\int 1 \mathrm{~d}\left(b_{p, r} v o l-\right.$ $\left.b_{p_{n}, r} \operatorname{vol}_{n}\right)$. Hence

$$
\leq(1+\varepsilon) \int 1 \mathrm{~d}\left(b_{p, r} v o l-b_{p_{n}, r} v_{o l}\right)
$$

This converges to zero as $n \rightarrow \infty$ by the definition of convergence of mm-spaces. Hence the spaces $M_{n} \sqcup M$ constructed in the proof of Theorem 1.3 also serve to prove convergence with respect to $\mathrm{PM}[k]$.

For the other direction take a sequence converging with respect to $d_{\mathrm{PM}[k]}$. Again we only treat the case $k=1$. Fix some $r, \varepsilon>0$. We have comparison spaces $L_{n}$ with embeddings $\tilde{\iota}_{n}: B_{n}:=\mathrm{B}\left[p_{n}, r\right] \rightarrow L_{n}$ and $\tilde{\eta}_{n}: B:=\mathrm{B}[p, r] \rightarrow L_{n}$ such that

$$
\begin{array}{r}
\varepsilon_{n}^{\prime}:=\sup _{f: L \rightarrow[-1,1]} \mathrm{d}_{\mathrm{H}}\left(\tilde{\iota}_{n} B_{n}, \tilde{\eta}_{n} B\right)+\left|\tilde{\iota}_{n}\left(p_{n}\right) \tilde{\eta}_{n}(p)\right|+\int f \mathrm{~d}\left(\tilde{\iota}_{n *} \operatorname{vol}_{n}-\tilde{\eta}_{n *} v o l\right) \\
\stackrel{n \rightarrow \infty}{\longrightarrow} 0
\end{array}
$$

where the supremum is taken over all Lipschitz functions with Lipschitz constant not greater than one. Again from the proof of Theorem 1.3 we already have comparison maps $\iota_{n}: \mathrm{B}\left[p_{n}, r\right] \rightarrow M$ satisfying (1.3) for $n$ sufficiently large. By Lemma A.1 we can without loss of generality (replace $3 \varepsilon$ by $\varepsilon$ ) assume that these comparison maps are measurable and, moreover, that

$$
\left|\tilde{\eta}_{n}\left(\iota_{n}(x)\right) \tilde{\iota}_{n}(x)\right|<\varepsilon
$$

By a diagonal argument we may also assume that there is actually a sequence $\varepsilon_{n} \rightarrow 0$ as $n \rightarrow \infty$ such that 1.3 holds with $\varepsilon$ replaced by $\varepsilon_{n}$. Now given any bounded continuous function $f: M \rightarrow \mathbb{R}$ we have to show that

$$
\int f \mathrm{~d} \iota_{n *}\left(b_{p_{n}, r} v o l_{n}\right) \rightarrow \int f \mathrm{~d} b_{p, r} v o l
$$

as $n \rightarrow \infty$. Since $M$ is proper, we may assume that $f$ is compactly supported. Hence we can approximate $f$ by Lipschitz functions with respect to the uniform norm [Dud02, Theorem 11.2.4]. With out loss of generality we can therefore assume that $f$ is a Lipschitz function. By scaling $f$ with a positive real factor we can also assume that the Lipschitz constant of $f$ is bounded by one. Further note that any Lipschitz function extends from an arbitrary subspace to the whole space preserving the Lipschitz constant McS34, Theorem 1] and, hence, there 
are extensions $\bar{f}$ of $f \circ \tilde{\eta}_{n}^{-1}$ and $\bar{b}_{p, r}$ of $b_{p, r} \circ \tilde{\eta}_{n}^{-1}$ to the whole of $L_{n}$. We estimate

$$
\begin{array}{rl}
\mid \int f & \mathrm{~d} \iota_{n *}\left(b_{p_{n}, r} v l_{n}\right)-b_{p, r} v o l \mid \\
& \leq\left|\int f \mathrm{~d} \iota_{n *}\left(b_{p_{n}, r} v o l_{n}\right)-b_{p, r} \iota_{n *} v o l_{n}\right|+\left|\int f \mathrm{~d} b_{p, r} \iota_{n *} v o l_{n}-b_{p, r} v o l\right| \\
& =\left|\int\left(f \circ \iota_{n}\right) \cdot\left(b_{p_{n}, r}-b_{p, r} \circ \iota_{n}\right) \mathrm{d} v o l_{n}\right|+\left|\int f \cdot b_{p, r} \mathrm{~d} \iota_{n *} v o l_{n}-v o l\right| \\
& \leq \varepsilon_{n} \int_{B_{n}} 1 \mathrm{~d} v o l_{n}+\left|\int \bar{f} \cdot \bar{b}_{p, r} \mathrm{~d}\left(\tilde{\eta}_{n} \circ \iota_{n}\right)_{*} v o l_{n}-\tilde{\eta}_{n *} v o l\right| \\
& \leq 2 \varepsilon_{n} \int_{B_{n}} 1 \mathrm{~d} v_{n}+\left|\int \bar{f} \cdot \bar{b}_{p, r} \mathrm{~d} \tilde{\iota}_{n *} v_{n} l_{n}-\tilde{\eta}_{n *} v o l\right| \\
& \leq 2 \varepsilon_{n} \int_{B_{n}} 1 \mathrm{~d} \operatorname{vol}_{n}+\varepsilon_{n}^{\prime} .
\end{array}
$$

The integral is bounded, as is apparent from looking at $d_{\mathrm{PM}^{\prime}-\text { convergence for a }}$ larger ball, let's say $\mathrm{B}[p, r+1]$. This proves that $M_{n}$ converges to $M$ in sense of (1.11) by the $\iota_{n}$ constructed.

Completeness. Take any (Cauchy) $d_{\mathrm{PM}}$-convergent sequence $M_{n}$. As we are in a metric space, it is sufficient to find a subsequence converging to some $M$. Is is also sufficient that this convergence takes place in the sense of $(1.11)$. We will first prove the claim in case $k=1$, i.e. for mm-spaces with one measure. Fix any $r>0$ and $0<\varepsilon<1$. By Theorem 1.3 there is a PGH-limit $M$ of this sequence given by some comparison maps $\iota_{n}: \mathrm{B}\left[p_{n}, r\right] \rightarrow M$ subject to condition (1.3). By Lemma A.1 we may assume that the $\iota_{n}$ 's are measurable. Moreover there has to be a bound $m=m_{r, \varepsilon}$ on the total measure of $m_{n}=m_{n, r, \varepsilon}:=b_{p_{n}, r} v_{n} l_{n}\left(M_{n}\right)$ because otherwise there would be a diverging subsequence of $m_{n}$, and the definition of $d_{\mathrm{PM}}^{\prime}$ the corresponding subsequence of $M_{n}$ 's would not be bounded.

We can do this argument for any $r=1,2, \ldots$. By a diagonal argument we can find a subsequence $(n(r))_{r=1}^{\infty}$ and comparison maps $\iota_{n}: \mathrm{B}\left[p_{n(r)}, r\right] \rightarrow M$. The resulting sequence has the property that for any $s=1,2, \ldots$ the volume of the balls $\mathrm{B}[p, s]$ is bounded by $m_{s+1, \varepsilon}$ with respect to the measures $\iota_{n(r) *} b_{p_{n}, r} v o l_{n}$ for sufficiently large $r$. Hence the sequence $\iota_{n(r) *} b_{p_{n}, r} \operatorname{vol}_{n}$ subconverges for $r \rightarrow \infty$ with respect to weak ${ }^{\#}$ convergence DV03, Proposition A2.6.IV]. The corresponding subsequence of $M_{n}$ and $\iota_{n}$ 's is what we looked for.

Separability. First take a countable dense subset $\left\{M_{i}\right\}_{i}$ of PM. Then take for each $M_{i}$ a dense subset $\operatorname{vol}_{i j}$ of $\mathcal{M}\left(M_{i}\right)$, which exists as $\mathcal{M}\left(M_{i}\right)$ is separable DV03, Theorem A2.6.III(i)]. We show that

$$
\left\{\left(M_{i}, p_{i}, \operatorname{vol}_{i j_{1}}, \ldots, \operatorname{vol}_{i j_{k}}\right)\right\}_{i, j_{1}, \ldots, j_{k}}
$$

is dense in $\mathrm{PM}[k]$ : Given $M=\left(M, p\right.$, vol $_{1}, \ldots$, vol $\left._{k}\right) \in \mathrm{PM}$ choose a radius $r^{\prime}$ such that $d_{\mathrm{PM}}\left(\mathrm{B}\left[p, r^{\prime}\right], M\right)<\varepsilon^{\prime}$ where

$$
M^{\prime}=\left(M^{\prime}, p, \operatorname{vol}_{1}^{\prime}, \ldots, \operatorname{vol}_{k}^{\prime}\right):=\left(\mathrm{B}\left[p, r^{\prime}\right], p,\left.\operatorname{vol}_{1}\right|_{\mathrm{B}\left[p, r^{\prime}\right]}, \ldots,\left.\operatorname{vol}_{k}\right|_{\mathrm{B}\left[p, r^{\prime}\right]}\right) .
$$

Choose $M_{i} \in \mathrm{PM}$ such that $\mathrm{d}_{\mathrm{PGH}}\left(M^{\prime}, M_{i}\right)<\varepsilon / 2$ for some $\varepsilon$ such that $\varepsilon$. $\operatorname{vol}\left(M^{\prime}\right)<\varepsilon^{\prime}$. By the same construction as in the second part of this proof this 
implies that there is a comparison space $L$ and a measurable $\varepsilon$-distortion $\iota$ from $M^{\prime} \subset L$ to $\mathrm{B}\left[p_{i}, r^{\prime}\right] \subset L$. Now view $\iota$ as a map from $\mathrm{B}\left[p_{i}, r^{\prime}\right] \subset L$ to $\mathrm{B}\left[p, r^{\prime}\right] \subset L$. Choose vol $_{i j_{1}}, \ldots$, vol $_{i j_{k}}$ such that

$$
d_{\mathrm{PM}}\left(\left(M_{i}, p_{i}, \iota_{*} \operatorname{vol}_{1}^{\prime}, p_{i}, \ldots, \iota_{*} \operatorname{vol}_{k}^{\prime}\right),\left(M_{i}, p_{i}, \operatorname{vol}_{i j_{1}}, \ldots, \operatorname{vol}_{i j_{k}}\right)\right)<\varepsilon .
$$

This implies $\int_{L} f \mathrm{~d}\left(v l^{\prime}-\operatorname{vol}_{i j}\right) \leq \int_{L} f \mathrm{~d}\left(\left(v o l^{\prime}-\iota_{*} v o l^{\prime}\right)+\left(\iota_{*} v o l^{\prime}-v_{o l} l_{i j}\right)\right) \leq$ $\varepsilon\left(\operatorname{vol} M^{\prime}\right)+\varepsilon^{\prime}<2 \varepsilon^{\prime}$. Hence

$$
\begin{aligned}
d_{\mathrm{PM}} & \left(M, p_{i},\left(M_{i}, \operatorname{vol}_{i j_{1}}, \ldots, \operatorname{vol}_{i j_{k}}\right)\right) \\
& \leq d_{\mathrm{PM}}\left(M, M^{\prime}\right)+d_{\mathrm{PM}}\left(M^{\prime},\left(M_{i}, p_{i}, \operatorname{vol}_{i j_{1}}, \ldots, \operatorname{vol}_{i j_{k}}\right)\right) \\
& \leq \varepsilon^{\prime}+\sum_{r=1}^{\infty} r^{-2} \cdot\left(\varepsilon / 2+2 \varepsilon^{\prime}\right) .
\end{aligned}
$$

This proves that $\left(M_{i}, p_{i}, \operatorname{vol}_{i j}\right)$ can be chosen arbitrarily close to $M$.

\section{A.3 Space of field spaces}

In this section we prove only metrizability of the tensor norm. To this end we recall a bit of set point topology. From Schubert Sch75, §§ I.8.3, I.9.1, I.9.3 Satz 2] we have:

Definition A.2 (normal space). A topological space $\mathcal{X}$ is called normal if it is Hausdorff and any two disjoint closed sets have disjoint neighborhoods.

Definition A.3 (completely regular space). A topological space $\mathcal{X}$ is called completely regular if it is Hausdorff and to every point $x \in \mathcal{X}$ and any neighborhood $U$ of $x$ there is a continuous function $\mathcal{X} \rightarrow[0,1]$ vanishing on $x$ and 1 on the complement of $U$

Theorem A.4 (Urysohn metrization theorem). For a second countable topological space the following are equivalent:

- $\mathcal{X}$ is metrizable;

- $\mathcal{X}$ is normal;

- $\mathcal{X}$ is completely regular.

Corollary A.5. If a topological space $\mathcal{Y}$ is Hausdorff and the quotient of a metrizable compact space $\mathcal{X}$, then it is metrizable.

Proof. Recall that $\mathcal{Y}$ is a quotient of $\mathcal{X}$ under $f: \mathcal{X} \rightarrow \mathcal{Y}$ if $f$ is surjective and $\mathcal{Y}$ has the finest topology such that $f$ is continuous. This is equivalent to the property that $V \subset \mathcal{Y}$ is open if and only if $f^{-1}(\mathcal{V})$ is open, or the property that $V \subset \mathcal{Y}$ is closed if and only if $f^{-1}(\mathcal{V})$ is closed Sch75, $\S$ I.4.3 Satz 1]. The space $\mathcal{Y}=f(\mathcal{X})$ is compact being the image of a compact space. Moreover, if a set $U \subset \mathcal{X}$ is open, so is its image $f(U)$, because if $U$ is open, then the complement $U^{\mathrm{C}}$ is closed, hence $U^{\mathrm{C}}$ is compact, hence $f\left(U^{\mathrm{C}}\right)=f(U)^{\mathrm{C}}$ is compact, hence $f(U)^{\mathrm{C}}$ is closed (since $\mathcal{Y}$ Hausdorff), hence $f(U)$ open.

A countable base of $\mathcal{Y}$ is given by the image of a countable base of $\mathcal{X}$ : First note that these images are open. For any open set $V \subset \mathcal{Y}$ the preimage $f^{-1}(V)$ is a union of sets from the countable base of $\mathcal{X}$. Hence $V$ is a union of the image of these sets. As Hausdorff compact space $\mathcal{Y}$ is normal [Sch75, § I.8.2 Satz 1]. Hence $\mathcal{Y}$ is metrizable by the Urysohn metrization theorem. 
Definition 1.26. $\left[\mathrm{C}^{m^{\prime}, \alpha^{\prime}}-\mathrm{C}^{m, \alpha}-\Theta\right.$-norm on the scale of $\left.\varrho\right]$ For $\alpha \in(0,1], \beta \in$ $(0, \alpha)$, and $k, l$ non-negative integers. For a section $s \in \mathrm{C}^{m^{\prime}, \alpha^{\prime}}\left(M, \mathrm{~T}^{k, l} M\right)$ over a manifolds $M \in \mathrm{P} \mathcal{M}_{\mathrm{C}^{m, \alpha} \leq \Theta}^{d, \varrho}$ the $\mathrm{C}^{m^{\prime}, \alpha^{\prime}}-\mathrm{C}^{m, \alpha}-\Theta$-norm on the scale of $\varrho$ of $(M, p)$ is defined as

$$
\inf \left\{\left\|\varphi^{*} s\right\|_{\mathrm{C}^{m^{\prime}, \alpha^{\prime}}} \mid \varphi:\left(\mathrm{B}_{\mathbb{R}^{n}}(0, \varrho), 0\right) \rightarrow(U, p) \subset M \quad \text { with }\|\varphi\|_{\mathrm{C}^{m, \alpha}} \leq \Theta\right\} .
$$

The $\mathrm{C}^{m^{\prime}, \alpha^{\prime}}-\mathrm{C}^{m, \alpha}-\Theta$-norm on the scale of $\varrho$ of a non-pointed space is defined as the supremum of all norms of spaces $(M, x, s)$ for all $x \in M$, like for the metric tensor. Let

$$
\mathrm{T}_{\mathrm{C}^{\mathrm{m}^{\prime}, \alpha^{\prime}} \leq \Theta^{\prime}}^{\mathrm{k}, \mathrm{P}} \mathcal{M}_{\mathrm{C}^{m, \alpha} \leq \Theta}^{d, \varrho} \quad \text { and } \quad \mathrm{T}_{\mathrm{C}^{\mathrm{m}^{\prime}, \alpha^{\prime}} \leq \Theta^{\prime}}^{\mathrm{k}, \mathrm{P}} \mathcal{M}_{\mathrm{C}^{m, \alpha} \leq \Theta}^{d, \varrho \text { har }}, \text { resp. }
$$

be the space of all (equivalence classes of) pointed Riemannian manifolds with sections $(M, p, s)$ such that $\|M\|_{\mathrm{C}^{m, \alpha}}^{d, \varrho} \leq \Theta$ and $\|M\|_{\mathrm{C}^{m, \alpha}}^{d, \varrho \text { har }} \leq \Theta$, resp., and $(M, s)$ has $\mathrm{C}^{m^{\prime}, \alpha^{\prime}}-\mathrm{C}^{m, \alpha}-\Theta$-norm on the scale of $\varrho$ not greater than $\Theta^{\prime}$.

Lemma A.6. Let $0 \leq \beta<\alpha$ and $0 \leq \beta^{\prime}<\alpha^{\prime}$ with $\beta^{\prime}<\alpha$. The space

$$
\mathcal{A}:=\mathrm{T}_{\mathrm{C}^{m^{\prime}, \alpha^{\prime}} \leq \Theta^{\prime}}^{\mathrm{k}, \mathrm{P}} \mathcal{M}_{\mathrm{C}^{m, \alpha} \leq \Theta}^{d, \varrho}
$$

is sequentially compact with respect to the $\mathrm{C}^{m^{\prime}, \beta^{\prime}}-\mathrm{C}^{m, \beta}$-topology.

Proof. Given any sequence $\left(M_{n}, s_{n}\right)$ of spaces in $\mathcal{A}$ we first choose a subsequence converging to some $M$ as possible by the Fundamental Theorem of convergence Theory, Theorem 1.24. For each radius $r>e^{\Theta}\left(e^{\Theta} \varrho+1\right)$ fix some comparison maps $\iota_{n}: \Omega_{n} \rightarrow M$ according to Definition 1.19 Choose an atlas covering $\mathrm{B}\left(p, r e^{-\Theta}-e^{\Theta} \varrho-1\right)$ with finitely many charts $\varphi_{i}$, indexed by the finite set $I$, of $\mathrm{C}^{m, \alpha}$-norm on the scale of $\varrho$ not greater than $\Theta$; by choice of parameters the ranges of these charts are contained in the ranges of the comparison maps for sufficiently large $n$. The metrics

$$
\begin{aligned}
\left(\varphi_{i}^{*} \iota_{n *} s_{n}\right)_{\lambda_{1}, \ldots, \lambda_{k}}^{\mu_{1}, \ldots, \mu_{l}}=\sum_{\substack{\lambda_{1}^{\prime}, \ldots, \lambda_{k}^{\prime} \\
\mu_{1}^{\prime}, \ldots, \mu_{l}^{\prime}}} s_{n}\left(\iota_{n} \varphi_{i}(\xi)\right)_{\substack{\lambda_{1}^{\prime}, \ldots, \lambda_{k}^{\prime} \\
\mu_{1}^{\prime}, \ldots, \mu_{l}^{\prime}}\left(\iota_{n} \circ \varphi_{i}\right)_{, \lambda_{1}} \cdot \ldots \cdot\left(\iota_{n} \circ \varphi_{i}\right)_{, \lambda_{k}}} \cdot\left(\iota_{n} \circ \varphi_{i}\right)_{, \mu_{1}} \circ \varphi_{i j} \cdot \ldots \cdot\left(\iota_{n} \circ \varphi_{i}\right)_{, \mu_{k}} \circ \varphi_{i j}
\end{aligned}
$$

are bounded in $\mathrm{C}^{\alpha \wedge \alpha^{\prime}}$-norm since $\left(\iota_{n} \circ \varphi_{i}\right)^{-1}$ and $\left(\iota_{n} \circ \varphi_{i}\right)$ are bounded in $\mathrm{C}^{1, \alpha}{ }_{-}$ norm by Theorem 1.21 and (1.24), and products and concatenations of Hölder bounded function are again Hölder bounded by Corollary 1.11 and Lemma 1.13 For each $i$ we choose inductively a subsequence of the former subsequence of $M_{n}$ 's such that on these charts $\varphi_{i}^{*} \iota_{n *} s_{n} \mathrm{C}^{\beta^{\prime}}$-converges. Repeating this process for a sequence $r_{j} \rightarrow \infty$ choosing further and further subsequences. A diagonal argument gives a convergent subsequence on $M_{n}$.

To check that this is indeed a tensor on each $\mathrm{B}\left(p, r_{j} e^{-\Theta}-e^{\Theta} \varrho-1\right)$, we have to check that the limits of the $\varphi_{i}^{*} \iota_{n *} s_{n}$ 's are invariant under chart transition. But since $s_{n}$ is invariant under chart transition, we already have

$$
\begin{aligned}
\left(\varphi_{i}^{*} \iota_{n *} s_{n}\right)_{\lambda_{1}, \ldots, \lambda_{k}}^{\mu_{1}, \ldots, \mu_{l}}=\sum_{\substack{\lambda_{1}^{\prime}, \ldots, \lambda_{k}^{\prime} \\
\mu_{1}^{\prime}, \ldots, \mu_{l}^{\prime}}}\left(\varphi_{j}^{*} \iota_{n *} s_{n}\right)_{\lambda_{1}^{\prime}, \ldots, \lambda_{k}^{\prime}}^{\mu_{1}^{\prime}, \ldots, \mu_{l}^{\prime}} \cdot \varphi_{i j, \lambda_{1}} \cdot \ldots \cdot \varphi_{i j, \lambda_{k}} \\
\cdot \varphi_{j i, \mu_{1}} \circ \varphi_{i j} \cdot \ldots \cdot \varphi_{i j, \mu_{k}} \circ \varphi_{i j}
\end{aligned}
$$

on suitable domains of definition. Hence these equations hold for the limits as well. 
Lemma 1.27. Let $0 \leq \beta<\alpha$ and $0 \leq \beta^{\prime}<\alpha^{\prime}$ with $\beta^{\prime}<\alpha$. The space

$$
\mathcal{A}:=\mathrm{T}_{\mathrm{C}^{m^{\prime}, \alpha^{\prime}} \leq \Theta^{\prime}}^{\mathrm{k}, \mathrm{P}} \mathcal{M}_{\mathrm{C}^{m, \alpha} \leq \Theta}^{d, \varrho}
$$

is completely metrizable and compact in the $\mathrm{C}^{m^{\prime}, \beta^{\prime}}-\mathrm{C}^{m, \beta}$-topology.

Proof. It suffices to prove metrizability on $\mathcal{A}$ : Any sequentially compact metrizable space is compact $[\mathrm{Sch} 75, \S$ I.7.4, Satz 4]. Hence by Lemma A.6 the space $\mathcal{A}$ is compact. Thus $\mathcal{A}$ is a compact, and hence a closed subset of its metric completion. Therefore $\mathcal{A}$ coincides with its completion. Metrization will be achieved by Urysohn's theorem.

For any space $M \in \mathrm{P}_{\mathrm{C}^{m, \alpha} \leq \Theta}^{d, \varrho}$ choose some charts $\left\{\varphi_{i}: \mathrm{B}(0, \varrho) \rightarrow M\right\}_{i \in I}$ with $\|\varphi\|_{\mathrm{C}^{m, \alpha}}^{\varrho} \leq \Theta$ for some index set $I$. Let $I_{r, M}$ be the set of all indices $i$ such that $\varphi_{i}(\mathrm{~B}(0, \varrho)) \cap \mathrm{B}[p, r] \neq \emptyset$. Let $\Omega_{r, M}:=\bigcup_{i \in I_{r, M}} \varphi_{i}(\mathrm{~B}(0, \varrho))$. Set

$$
U_{r, \varepsilon}(M):=\left\{M^{\prime} \in \mathcal{M}_{\mathrm{C}^{m, \alpha} \leq \Theta}^{d, \varrho} \mid \exists \iota: \Omega_{r, M} \rightarrow M^{\prime} \text { s.t. }\left\|\varphi_{i}^{*} \iota^{*} g^{\prime}-\varphi_{i}^{*} g\right\|_{\mathrm{C}^{m, \alpha}}<\varepsilon\right\}
$$

where the condition holds for all $i \in I_{r, M}$ and $\iota$ is a smooth map. These sets are open by definition of $\mathrm{C}^{m, \alpha} \leq \Theta$-convergence because for the metrics $\varphi_{i}^{*} \iota^{*} \iota_{n *} g_{n}$ restricted to suitable domains of definition corresponding to a chart $\psi: \mathrm{B}(0, \varrho) \rightarrow M^{\prime}$ with $\|\psi\|_{\mathrm{C}^{m, \alpha}}^{\varrho} \leq \Theta$

$$
\begin{aligned}
\limsup _{n \rightarrow \infty} & \left\|\varphi_{i}^{*} \iota^{*} \iota_{n *} g_{n}-\varphi_{i}^{*} g\right\|_{\mathrm{C}^{\alpha}} \\
& \leq \limsup _{n \rightarrow \infty}\left\|\varphi_{i}^{*} \iota^{*} \iota_{n *} g_{n}-\varphi_{i}^{*} \iota^{*} g^{\prime}\right\|_{\mathrm{C}^{\alpha}}+\left\|\varphi_{i}^{*} \iota^{*} g^{\prime}-\varphi_{i}^{*} g\right\|_{\mathrm{C}^{\alpha}} \\
& <\limsup _{n \rightarrow \infty}\left\|\varphi_{i}^{*} \iota^{*} \iota_{n *} g_{n}-\varphi_{i}^{*} \iota^{*} g^{\prime}\right\|_{\mathrm{C}^{\alpha}}+\varepsilon \\
& =\varepsilon+\limsup _{n \rightarrow \infty}\left\|(\underbrace{\varphi_{i} \circ \iota \circ \psi^{-1}}_{=: \tilde{\iota}_{i, \psi}})^{*}(\underbrace{\psi^{*} \iota_{n *} g_{n}-\psi^{*} g^{\prime}}_{=: \Delta_{n} g})\right\|_{\mathrm{C}^{\alpha}} \\
& \quad{ }_{\text {Corollary } 1.15}^{\leq} \limsup _{n \rightarrow \infty} C\left(\left\|\Delta_{n} g\right\|_{\mathrm{C}^{\alpha}}\left\|\tilde{\iota}_{i, \psi}\right\|_{\mathrm{C}^{\alpha}}+\left\|\Delta_{n} g\right\|_{\mathrm{C}^{0}}\right)\left\|\tilde{\iota}_{i, \psi}\right\|_{\mathrm{C}^{1, \alpha}}^{2}
\end{aligned}
$$

since $M_{n}$ converges to $M^{\prime}$ and hence $\left\|\Delta_{n} g\right\|_{\mathrm{C}^{\alpha}}=\left\|\psi^{*} \iota_{n *} g_{n}-\psi^{*} g^{\prime}\right\|_{\mathrm{C}^{\alpha}} \stackrel{n \rightarrow \infty}{\longrightarrow} 0$

$$
=\varepsilon+0 .
$$

Observe that for $M^{\prime} \in U_{r, \varepsilon}(M)$ we can embed $\mathrm{B}[p, r]$ and $\mathrm{B}\left[p^{\prime}, r\right]$ into

$$
L:=\left(\Omega \times[0, \varepsilon],\left(1-\frac{t}{\varepsilon}\right) g+\frac{t}{\varepsilon} \iota^{*} g^{\prime}+\mathrm{d} t\right)
$$

along the maps $x \mapsto(x, 0)$ and $x \mapsto(x, \varepsilon)$. From this we read for $r \in \mathbb{N}$

$$
\begin{array}{r}
d_{\mathrm{PM}}^{\prime}\left(\mathrm{B}[p, r], \mathrm{B}\left[p^{\prime}, r\right]\right) \leq \mathrm{d}_{\mathrm{H}}\left(\mathrm{B}[p, r] \times\{0\},\left(\iota^{-1} \mathrm{~B}\left[p^{\prime}, r\right]\right) \times\{\varepsilon\}\right)+|(p, 0)(p, \varepsilon)|_{L} \\
+\sup _{f: L \rightarrow[-1,1]} \int f(x, 0) \mathrm{d} v o l-\int f\left(\iota^{-1}(x), \varepsilon\right) \mathrm{d} v o l^{\prime}
\end{array}
$$

where $f$ is a Lipschitz function with Lipschitz constant not greater than 1 . Each summand can be bounded as follows:

$$
\begin{aligned}
\mathrm{d}_{\mathrm{H}}\left(\mathrm{B}[p, r] \times\{0\},\left(\iota^{-1} \mathrm{~B}\left[p^{\prime}, r\right]\right) \times\{\varepsilon\}\right) \\
\leq \mathrm{d}_{\mathrm{H}}\left(\mathrm{B}[p, r] \times\{0\},\left(\iota^{-1} \mathrm{~B}\left[p^{\prime}, r\right]\right) \times\{0\}\right) \\
\quad+\mathrm{d}_{\mathrm{H}}\left(\left(\iota^{-1} \mathrm{~B}\left[p^{\prime}, r\right]\right) \times\{0\},\left(\iota^{-1} \mathrm{~B}\left[p^{\prime}, r\right]\right) \times\{\varepsilon\}\right) \\
\leq \varepsilon r+\varepsilon,
\end{aligned}
$$


by a geodesic comparison.

$$
\begin{aligned}
& |(p, 0)(p, \varepsilon)|_{L} \leq \varepsilon, \text { and } \\
& \qquad f(x, 0) \mathrm{d} v o l-\int f\left(\iota^{-1}(x), 0\right) \mathrm{d} v o l^{\prime} \\
& \quad \leq \int f(x, 0)-f(x, \varepsilon) \mathrm{d} v o l+\int f(x, \varepsilon) \mathrm{d} v o l-\int f\left(\iota^{-1}(x), \varepsilon\right) \mathrm{d} v o l^{\prime} \\
& \quad \leq \operatorname{vvol}(\Omega)+\int_{\Omega} f(x, \varepsilon) \mathrm{d}\left(\operatorname{vol}-\left(\iota^{-1}\right)_{*} \operatorname{vol} l^{\prime}\right) \\
& \quad \leq \operatorname{vvol}(\Omega)+\sum_{i \in I_{r, M}} \int\left|\sqrt{\left|\operatorname{det} \varphi_{i}^{*} g\right|}-\sqrt{\left|\operatorname{det} \varphi_{i}^{*}\left(\iota^{-1}\right)^{*} g^{\prime}\right|}\right| \mathrm{d} x \\
& \quad \leq \operatorname{vvol}(\Omega)+C\left\|\varphi_{i}^{*} \iota^{*} g^{\prime}-\varphi_{i}^{*} g\right\|_{\mathrm{C}^{\alpha}}
\end{aligned}
$$

where $C$ is a constant depending only on $\varrho$ and $d$, given by standard estimates Corollary 1.11 and Lemma 1.13 These bounds imply that the sets $U_{r, \varepsilon}(M)$ form a neighborhood base of $M$, i.e. any sequence $M_{i} \in \mathrm{P}_{\mathrm{C}^{m}, \alpha}^{d, \varrho} \leq \Theta$ converges to $M$ if and only if for every $\varepsilon>0$ and $r>0$ all but finitely many $M_{i}$ are in $U_{r, \varepsilon}(M)$.

Observe that every base $\mathcal{B}$ of a compact metric space $\mathcal{X}$ contains a countable base: A base of a topology contains a neighborhood base for each point. For each $x \in \mathcal{X}$ and $\varepsilon>0$ we can cover $\mathcal{X}$ with a finite number of opens $U_{\varepsilon, 1}, \ldots, U_{\varepsilon, N(\varepsilon)} \in$ $\mathcal{B}$ such that each of them is contained in the open $\varepsilon / 2$-ball around some point in $\mathcal{X}$. Hence every point $x \in \mathcal{X}$ is contained in at least one of these opens and every such open is contained in the $\varepsilon$-ball around $x$ due to triangle inequality. Hence the union $\mathfrak{B}:=\left\{U_{1 / n, k} \mid n=1,2,3, \ldots ; k=1, \ldots, N(1 / n)\right\}$ contains a neighborhood base of each point. Thus $\mathfrak{B}$ is a base of the topology. Moreover $\mathfrak{B}$ is countable.

We apply this observation to $\mathrm{P} \mathcal{M}_{\mathrm{C}^{m, \alpha}}^{d, \varrho} \leq \Theta$. This space is compact and metrizable due to Theorem 1.24 Hence the base given by $U_{r, \varepsilon}(M)$ for $\varepsilon>0, r>0$, and $M \in \mathrm{P} \mathcal{M}_{\mathrm{C}^{m, \alpha} \leq \Theta}^{d, \varrho}$ contains a countable base $\left\{U_{r_{a}, \varepsilon_{a}}\left(M_{a}\right)\right\}_{a \in \mathbb{N}}$ of the topology of $\mathrm{P}_{\mathrm{C}^{m, \alpha} \leq \Theta}^{d, \varrho}$. For each $M_{a}, r_{a}, \varepsilon_{a}$, and $\varphi_{a i}$ for $i \in I_{a}:=I_{M_{a}, r_{a}}$, we can choose a countable base $\left\{V_{a b}\right\}_{b \in \mathbb{N}}$ of the $\mathrm{C}^{m^{\prime}, \beta}$-topology on the metrizable and compact set $\mathrm{C}^{m^{\prime}, \beta^{\prime}}\left(\Omega, \mathrm{T}^{k, l} \Omega\right)$. Set $\Omega_{a}:=\bigcup_{i \in I_{a}} \varphi_{i}(\mathrm{~B}(0, \varrho))$. From this we define

$$
U_{a b}:=\left\{\begin{array}{l|l}
\left(M^{\prime}, s^{\prime}\right) \in \mathcal{A} \mid \begin{array}{l}
\exists \iota: \Omega_{a} \rightarrow M^{\prime} \text { such that } \\
\left\|\varphi_{i}^{*} \iota^{*} g^{\prime}-\varphi_{i}^{*} g\right\|_{\mathrm{C}^{m, \alpha}}<\varepsilon_{n} \text { and } \iota^{*} s^{\prime} \in V_{a b}
\end{array}
\end{array}\right\}
$$

where $\Omega$ is the union of all $\varphi_{i}(\mathrm{~B}(0, \varrho))$ with $i \in I_{r_{a}, M_{a}}$. Let $\left\{U_{j}\right\}_{j \in \mathbb{N}}$ be the base generated by $U_{a b}$ 's, and let $\mathcal{T}_{\left\{U_{j}\right\}}$ denote the topology generated by the $U_{a b}$ 's.

We observe that the notion on convergence induced by $\mathcal{T}_{\left\{U_{j}\right\}}$ is the same as $\mathrm{C}^{m^{\prime}, \alpha^{\prime}}-\mathrm{C}^{m, \alpha}$-convergence: If a sequence $\left(M_{n}, s_{n}\right)$ does $\mathrm{C}^{m^{\prime}, \alpha^{\prime}}-\mathrm{C}^{m, \alpha}$-converge to $(M, s) \in U_{a b}$ for some $a, b$ then, by construction there is a map $\iota: M_{a} \rightarrow M$ and moreover, by definition of $\mathrm{C}^{m^{\prime}, \alpha^{\prime}}-\mathrm{C}^{m, \alpha}$-convergence, for sufficiently large $n$ there are comparison maps from $\iota_{n}: \Omega_{n} \rightarrow M$ such that $\iota(\Omega) \subset \iota_{n}\left(\Omega_{n}\right)$. Since $\left(\iota_{n}^{-1}\right)^{*} g_{n}$ and $\iota_{n *} s$ converge to $g$ and $s$ as $n \rightarrow \infty$, we observe that $\left(M_{n}, s_{n}\right) \in U_{a b}$. For the other direction take some $(M, s)$ and a sequence $\left(M_{n}, s_{n}\right)$ such that for any $U_{a b}$ containing $(M, s)$ all but finitely many $\left(M_{n}, s_{n}\right)$ 's are in $U_{a b}$. Given any $r=1,2, \ldots$ we have to find (for all but finitely many $n$ ) comparison maps $\iota_{n}: \Omega_{n} \rightarrow M$ such that $\mathrm{B}\left(p_{n}, r\right) \subset \Omega_{n},\left(\iota_{n}^{-1}\right)^{*} g_{n} \rightarrow g$ in $\mathrm{C}^{m, \alpha}$, and $\iota_{n *} s_{n} \rightarrow s$ 
in $\mathrm{C}^{m^{\prime}, \alpha^{\prime}}$. To this end consider indices $a, b$ such that $\Omega_{a}$ contains $\mathrm{B}\left[p_{a}, r\right]$ and $M \in U_{a b}$. Choose a sequence of $a$ 's such that the $U_{a}$ 's form a neighborhood base of $M \in \mathcal{M}_{\mathrm{C}^{m, \alpha} \leq \Theta}^{d, \varrho}$. Further choose any $b=b(a)$ such that $(M, s) \in U_{a b}$ and $V_{a b}$ has diameter (see (1.1) ) less than 1/a. Let $a=a(j)$ be an ascending sequence of such $a$ 's. The definition of $U_{a(j) b(a(j))}$ give the required comparison maps $\iota_{n}: \Omega_{n} \rightarrow M$.

Next we check that the topology generated by the base $\left\{U_{j}\right\}_{j \in \mathbb{N}}$ is completely regular in the sense of Definition A.3. The topology generated by the base $\left\{U_{j}\right\}_{j \in \mathbb{N}}$ is Hausdorff: Let $(M, s)$ and $\left(M^{\prime}, s^{\prime}\right)$ be two distinct points. If $M \neq M^{\prime}$ then choose disjoint neighborhoods $U$ and $U^{\prime}$ of $M$ and $M^{\prime}$ in $\mathcal{M}_{\mathrm{C}^{m}, \alpha<\Theta}^{d, \varrho}$ and the sets $\left\{\left(M^{\prime \prime}, s\right) \mid M^{\prime \prime} \in U\right\}$ and $\left\{\left(M^{\prime \prime}, s\right) \mid M^{\prime \prime} \in U^{\prime}\right\}$. In case $M=M^{\prime}$ choose a radius $r$ such that the balls $(\mathrm{B}(p, r), s)$ and $\left(\mathrm{B}(p, r), s^{\prime}\right)$ are not isomorphic. Choose any base element $U_{r_{a}, \varepsilon_{a}}\left(M_{a}\right)$ of the topology of $\mathcal{M}_{\mathrm{C}^{m, \alpha}<\Theta}^{d, \varrho}$ as chosen above such that $r_{a}>r$. From the topology of $\mathrm{C}^{m^{\prime}, \beta^{\prime}}\left(\Omega, \mathrm{T}^{k, l} \Omega\right)$ choose base elements $V_{a b}$ and $V_{a b^{\prime}}$ that can distinguish $\left.s\right|_{\mathrm{B}(p, r)}$ and $\left.s^{\prime}\right|_{\mathrm{B}(p, r)}$. This proves that $\mathcal{A}$ is Hausdorff.

For the other property consider any point $(M, s) \in \mathcal{A}$. It is sufficient to prove the claim for any neighborhood $U_{a b}$ of $(M, s)$ because for any finite intersection $U_{a_{1} b_{1}} \cap \ldots \cap U_{a_{N} b_{N}}$ the required function $f$ is given by $f_{a_{1} b_{1}} \vee \ldots \vee f_{a_{N} b_{N}}$ where $f_{a_{1} b_{1}}, \ldots, f_{a_{N} b_{N}}$ are the corresponding functions for the subsets $U_{a_{1} b_{1}}, \ldots, U_{a_{N} b_{N}}$. Choose a neighborhood $U_{a^{\prime} b^{\prime}}$ of $(M, s)$ such that $\overline{U_{a^{\prime} b^{\prime}}} \subset U_{a b}$. It is sufficient to find a non-zero continuous function $f^{\prime}: \overline{U_{a^{\prime} b^{\prime}}} \rightarrow[0,1]$ such that $\left.f^{\prime}\right|_{\partial U_{a^{\prime} b^{\prime}}} \equiv 0$. Choose some $\iota: \Omega_{a} \rightarrow M$ and let $\varepsilon$ be such that $\mathrm{B}\left(\varphi_{i}^{*} \iota^{*} s, \varepsilon\right) \subset \varphi_{i}^{*}\left(V_{a^{\prime} b^{\prime}}^{a^{\prime} b^{\prime}}\right)$. On $\overline{U_{a^{\prime} b^{\prime}}}$ define

$$
f\left(M^{\prime \prime}, s^{\prime \prime}\right):=0 \vee\left(\varepsilon-\inf _{\iota^{\prime \prime}}\left\{\delta \mid\left\|\varphi_{i}^{*} \iota^{\prime \prime *} s^{\prime \prime}-\varphi_{i}^{*} s_{a}\right\|_{\mathrm{C}^{m^{\prime}, \alpha^{\prime}}} \forall i \in I_{a^{\prime}}\right\}\right)
$$

Continuity of this mapped is checked in parallel to the proof that a sequence converging with respect to the topology generated by the $U_{j}$ 's is a $\mathrm{C}^{m^{\prime}, \alpha^{\prime}}-\mathrm{C}^{m, \alpha_{-}}$ converging sequence. This proves that $\mathcal{A}$ is completely regular.

Now we are able to apply Urysohn's metrization Theorem A.4 to $\mathcal{T}_{\left\{U_{j}\right\}}$. Recall from Lemma 1.2 the fact that in a metric space a set $U$ is open if and only if for every sequence $\mathcal{T}_{\left\{U_{j}\right\}}$-converging to a point in $U$ all but finitely many members are already contained in $U$. Since $\mathcal{T}_{\left\{U_{j}\right\}}$-convergence coincides with $\mathrm{C}^{m^{\prime}, \beta^{\prime}}-\mathrm{C}^{m, \beta}$, this fact implies that the topology $\mathcal{T}_{\left\{U_{j}\right\}}$ coincides with the $\mathrm{C}^{m^{\prime}, \beta^{\prime}}-\mathrm{C}^{m, \beta}$-topology.

Definition 1.28. Let $k, l \geq 0$. The space of oriented pointed Riemannian manifolds with a $(k, l)$-field is the topological space

$$
\begin{aligned}
& \mathrm{T}_{\mathrm{C}^{m^{\prime}, \alpha^{\prime}} \leq \Theta^{\prime}}^{\mathrm{k}, \mathrm{P}} \stackrel{\mathcal{M}_{\mathrm{C}^{m, \alpha}}^{d, \varrho} \leq \Theta}{ }
\end{aligned}
$$

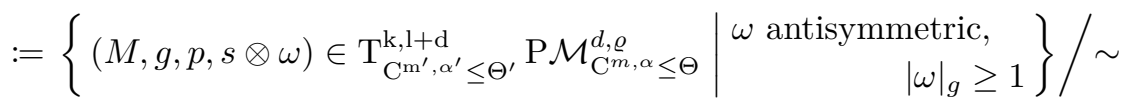

where $\sim$ is defined by $(M, g, p, s \otimes \omega) \sim\left(M^{\prime}, g^{\prime}, p^{\prime}, s^{\prime} \otimes \omega^{\prime}\right)$ if there is a diffeomorphism $f: M \rightarrow M^{\prime}$ such that

$$
\begin{aligned}
f(p) & =p^{\prime}, & g & =f^{*} g^{\prime} \\
f_{*} s & =s^{\prime}, & \left.f^{*}\left(\omega^{\prime}\right)\right|_{0} & =\left.\lambda \omega\right|_{0} \quad \text { for some } \lambda>0 .
\end{aligned}
$$


The space is given the quotient topology of the subspace topology of

$$
\left(\mathrm{T}_{\mathrm{C}^{m^{\prime}, \alpha^{\prime}} \leq \Theta^{\prime}}^{\mathrm{k}, 1+\mathrm{d}} \mathrm{P} \mathcal{M}_{\mathrm{C}^{m, \alpha} \leq \Theta}^{d, \varrho} \text {, topology induced by } \mathrm{C}^{m^{\prime}, \alpha^{\prime}}-\mathrm{C}^{m, \alpha}\right. \text {-convergence). }
$$

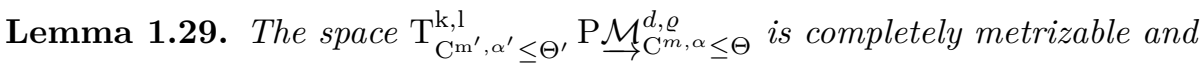

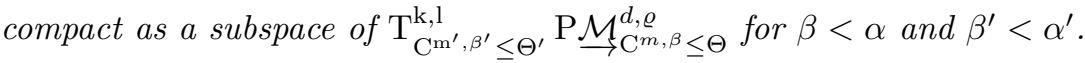

Proof. The space

$$
\mathcal{A}:=\left\{(M, g, p, s \otimes \omega) \in \mathrm{T}_{\mathrm{C}^{m^{\prime}, \alpha^{\prime}} \leq \Theta^{\prime}}^{\mathrm{k}, 1+\mathrm{d}} \mathrm{PM}_{\mathrm{C}^{m, \alpha} \leq \Theta}^{d, \varrho} \mid \begin{array}{r}
\omega \text { is antisymmetric, } \\
|\omega|_{g} \geq 1
\end{array}\right\}
$$

is compact being defined as a subspace of a compact space by closed conditions. Hence we have a sequence

$$
\mathcal{A} \rightarrow \mathcal{A} / \sim \stackrel{f}{\rightarrow} \mathrm{T}_{\mathrm{C}^{m^{\prime}, \alpha^{\prime}} \leq \Theta^{\prime}}^{\mathrm{k},} \mathrm{P} \mathcal{M}_{\mathrm{C}^{m, \alpha} \leq \Theta}^{d, \varrho}
$$

where the second map $f$ comes from forgetting $\omega$. We check the assumptions of Corollary A.5, i.e. the Hausdorff property.

Given two distinct points $M, M^{\prime} \in \mathcal{A} / \sim$ we immediately find disjoint open neighborhoods if their images under $f$ are distinct. Hence assume otherwise, i.e. $M^{\prime}=\left(M, g, p, s \otimes \omega^{\prime}\right)$ with $\left.\omega\right|_{0} \neq\left.\lambda \omega^{\prime}\right|_{0}$ (for all $\lambda>0$ ) after choice of representatives. Set $s^{\prime}:=s, \sigma:=s \otimes \omega$, and $\sigma^{\prime}:=s^{\prime} \otimes \omega^{\prime}$. By the assumption $M \neq M^{\prime}$ there has to be a radius $r>0$ and an open neighborhood $\Omega$ of $p$ containing $\mathrm{B}(p, r)$ such that there is no diffeomorphism $\iota: \Omega \rightarrow M$ such that

$$
\mathrm{B}(p, r) \subset \iota(\Omega), \quad \iota(p)=p, \quad \iota^{*} g=g, \text { and } \iota^{*} \sigma^{\prime}=\sigma .
$$

Therefore it is also possible to find neighborhoods $V$ and $V^{\prime}$ of $\sigma$ and $\sigma^{\prime}$, respectively, in $\mathrm{C}^{m^{\prime}, \beta}\left(\Omega, \mathrm{T}^{k, l+d} \Omega\right)$ such that there is no $\iota$ such that A.5 holds with $\sigma, \sigma^{\prime}$ replaced by some $\tilde{\sigma} \in V, \tilde{\sigma}^{\prime} \in V^{\prime}$ : Otherwise, we could choose a sequence of neighborhoods $V_{n}$ converging to $\{\sigma\}$ and $V_{n}^{\prime}$ converging to $\left\{\sigma^{\prime}\right\}$ together with sequences $\tilde{\sigma}_{n} \rightarrow \sigma, \tilde{\sigma}_{n}^{\prime} \rightarrow \sigma^{\prime}$, and maps $\iota_{n}: \Omega \rightarrow M$ fulfilling A.5 with $\tilde{\sigma}_{n}, \tilde{\sigma}_{n}^{\prime}$, and $\iota_{n}$ for each $n$. Due to $\iota_{n}^{*} g=g$ and Theorem 1.21)(ii) we have a uniform bound on the $\mathrm{C}^{1, \alpha}$-norm of the $\iota_{n}$ 's. Hence we can find a subsequence converging to some one-time differentiable function $\iota: \Omega \rightarrow M$ fulfilling A.5 in contradiction to our assumptions.

Let $\left\{\varphi_{i} \mid i \in I\right\}$ be a finite set of charts for $\Omega$. Define $U$ and $U^{\prime}$ by

$$
U^{(\prime)}:=\left\{\begin{array}{l|l}
(\tilde{M}, \tilde{\sigma}) \in \mathcal{A} & \begin{array}{c}
\exists \iota: \Omega \rightarrow \tilde{M} \text { such that for all } i \in I \\
\left\|\varphi_{i}^{*} \iota^{*} g^{\prime}-\varphi_{i}^{*} g\right\|_{\mathrm{C}^{m, \alpha}}<\varepsilon \text { and } \iota^{*} \tilde{\sigma} \in V^{(\prime)}
\end{array}
\end{array}\right\}
$$

for some $\varepsilon>0$. These sets are open by the same arguments as the sets A.4 are open. Moreover by construction these maps are disjoint. Thus their image in the quotient $\mathcal{A} / \sim$. Hence we can apply Corollary A.5 As in the lemmas above metrizability implies the remaining claims.

Definition 1.30. Let $\mathrm{T}_{\mathrm{C}^{m^{\prime}, \alpha^{\prime}} \leq \Theta^{\prime}}^{\mathrm{k}, \mathrm{P}} \mathcal{M}_{\mathrm{C}^{m, \alpha} \leq \Theta}^{d, \varrho\left[k^{\prime}, C\right]}, C>0$, denote the space of (equivalence classes of) spaces

$$
\left(M, g, p, s, \mu_{1}, \ldots, \mu_{k}\right)
$$


such that $(M, p, g, s) \in \mathrm{T}_{\mathrm{C}^{m^{\prime}, \alpha^{\prime}} \leq \Theta^{\prime}}^{\mathrm{k}, \mathrm{P}} \mathcal{M}_{\mathrm{C}^{m, \alpha} \leq \Theta}^{d, \varrho}$ and $\mu_{1}, \ldots, \mu_{k}$ are measure on $M$ satisfying the bound

$$
\mu_{i}(\mathrm{~B}[x, r]) \leq C^{r}+C
$$

for any $x \in M, r \geq 0$, and $i=1, \ldots, k$. On this space a notion of convergence is given by combination of the notion of convergence of $\mathrm{PM}^{[k+1]}$ and tensor convergence, namely, $\left(M_{n}, p_{n}, g_{n}, s_{n}, \mu_{n 1}, \ldots, \mu_{n k}\right)$ converges to $\left(M, p, g, s, \mu_{1}, \ldots, \mu_{k}\right)$ if and only if for every radius $r$, index $i=1, \ldots, k$ (and sufficiently large $n$ ) there are a domain $\Omega \supset \mathrm{B}(p, r)$ and (smooth) embeddings

$$
\iota_{n}: \Omega_{n} \rightarrow M
$$

from domains $\Omega_{n} \supset \mathrm{B}\left(p_{n}, r\right)$ such that

- $\left(\iota_{n}^{-1}\right)^{*} g_{n}$ converges in $\mathrm{C}^{m, \alpha}$ to $g$ on $\Omega$,

- $\iota_{n *} s_{n}$ converges in $\mathrm{C}^{m^{\prime}, \alpha^{\prime}}$ to $s$ on $\Omega$, and

- $\iota_{n *}\left(b_{p_{n}, r} \mu_{n i}\right)$ weak $\#$ converges to $b_{p, r} \mu_{i}$ for $i=1, \ldots, k$ and $b_{., .}$as defined in 1.10 .

Likewise, define

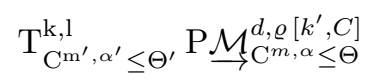

as a quotient of a subset of $\mathrm{T}_{\mathrm{C}^{m^{\prime}, \alpha^{\prime}} \leq \Theta^{\prime}}^{\mathrm{k}, \mathrm{l}+\mathrm{d}} \mathrm{P} \mathcal{M}_{\mathrm{C}^{m}, \alpha}^{d, \varrho\left[k^{\prime}, C\right]} \leq \Theta$ as in Definition 1.28 .

Lemma A.7. Lemma A.6 holds for $\mathrm{T}_{\mathrm{C}^{m^{\prime}, \alpha^{\prime}} \leq \Theta^{\prime}}^{\mathrm{k}, 1} \mathrm{PM}_{\mathrm{C}^{m}, \alpha}^{d, \varrho\left[k^{\prime}, C\right]}$ with respect to the topology defined in Definition 1.30, i.e. it is sequentially compact with respect to this topology:

Proof. First we observe that $\mathrm{P}_{\mathrm{C}^{m}, \alpha \leq \Theta}^{d, \varrho\left[k^{\prime}, C\right]}=\mathrm{T}_{\mathrm{C}^{m^{\prime}, \alpha^{\prime}} \leq \Theta^{\prime}}^{0,0} \mathrm{P}_{\mathrm{C}^{m}, \alpha}^{d, \varrho\left[k^{\prime}, C\right]}$ as a subset of $\mathbb{M}^{[k+1]}$ is compact: Given any sequence $\left(M_{n}, p_{n}, g_{n}, \mu_{n 1}, \ldots, \mu_{n k^{\prime}}\right)$ we can choose a converging subsequence of $\left(M_{n}, p_{n}, g_{n}\right)$ due to Theorem 1.24 . This means that (after going to a subsequence of the $M_{n}$ 's) for any radius there are embeddings $\iota_{r, n}: \Omega_{r, n} \rightarrow M$ for some space $M$ according to Definition 1.19 with $\mathrm{B}\left(p_{n}, r\right) \subset \Omega_{n}$. Due to [DV03, Corollary A2.6.V] there is a convergent subsequence of the measures $\iota_{n}^{*}\left(\left.\mu_{n i}\right|_{\mathrm{B}\left(p_{n}, r\right)}\right)$ weak ${ }^{\#}$ converging to some $\mu_{i r}$ for each $i=1, \ldots, k^{\prime}$. By the same theorem $\mu_{i r}$ weak ${ }^{\#}$ subconverges to some $\mu_{i}$ for $r=1,2, \ldots \rightarrow \infty$ and each $i=1, \ldots, k^{\prime}$. Since $\mathcal{M} M$ is a metrizable DV08, Proposition 9.1.IV(i)], we can use a diagonal argument to find a subsequence $\left(\iota_{r(m), n(m)}\right)_{m}$ of the $\iota_{r, n}$ 's such that $\left.\iota_{r\left(n^{\prime}\right), n\left(n^{\prime}\right)}^{*} \mu_{i}\right|_{\mathrm{B}\left(p_{n}, r\left(n^{\prime}\right)\right)}$ converges to $\mu$ for $n^{\prime} \rightarrow \infty$ and each $i=1, \ldots, k^{\prime}$. By this procedure we found a subsequence $\left(M_{n\left(n^{\prime}\right)}, p_{n\left(n^{\prime}\right)}, g_{n\left(n^{\prime}\right)}, \mu_{1 n\left(n^{\prime}\right)}, \ldots, \mu_{k^{\prime} n\left(n^{\prime}\right)}\right)$ which converges to $\left(M, p, g, \mu_{1}, \ldots, \mu_{k^{\prime}}\right)$ as $n^{\prime} \rightarrow \infty$.

The proof of Lemma A.6 applies mutatis tutandis by replacing the space $\mathrm{P} \mathcal{M}_{\mathrm{C}^{m, \alpha} \leq \Theta}^{d, \varrho}$ by $\mathrm{P} \mathcal{M}_{\mathrm{C}^{m, \alpha} \leq \Theta}^{d, \varrho\left[k^{\prime}, C\right]} \subset \mathbb{M}^{[k+1]}$ because compactness of $\mathrm{P} \mathcal{M}_{\mathrm{C}^{m, \alpha} \leq \Theta}^{d, \varrho}$ was the only property of this space used. This finishes the proof.

Lemma 1.31. Lemma 1.27 holds for the spaces $\mathrm{T}_{\mathrm{C}^{m^{\prime}, \alpha^{\prime}} \leq \Theta^{\prime}}^{\mathrm{k}, 1} \mathrm{P} \mathcal{M}_{\mathrm{C}^{m}, \alpha}^{d, \varrho\left[k^{\prime}, C\right]}$ and $\mathrm{T}_{\mathrm{C}^{m^{\prime}, \alpha^{\prime}} \leq \Theta^{\prime}}^{\mathrm{k}, 1} \stackrel{\mathrm{P}}{\left.\mathcal{M}_{\mathrm{C}^{m}, \alpha}^{d, \varrho} \leq k^{\prime}, C\right]}$, i.e. they are compact and completely metrizable as a subspace of $\mathrm{T}_{\mathrm{C}^{m^{\prime}, \beta^{\prime}} \leq \Theta^{\prime}}^{\mathrm{k}, 1} \mathrm{P} \mathcal{M}_{\mathrm{C}^{m, \beta} \leq \Theta}^{d, \varrho\left[k^{\prime}, C\right]}$ and $\mathrm{T}_{\mathrm{C}^{m^{\prime}, \beta^{\prime}} \leq \Theta^{\prime}}^{\mathrm{k}, \mathrm{P}} \stackrel{\mathcal{M}_{\mathrm{C}^{m}, \beta}^{d, \varrho} \leq \Theta}{\left.d k^{\prime}, C\right]}$, resp., for $\beta<\alpha$ and $\beta^{\prime}<\alpha^{\prime}$. 
Proof. For $\mathrm{T}_{\mathrm{C}^{\mathrm{m}^{\prime}, \alpha^{\prime}} \leq \Theta^{\prime}}^{\mathrm{k}, \mathrm{P}} \mathcal{M}_{\mathrm{C}^{m}, \alpha}^{d, \varrho\left[k^{\prime}, C\right]}$ the proof of Lemma 1.27 applies mutatis tutandis by replacing the space $\mathrm{P} \mathcal{M}_{\mathrm{C}^{m, \alpha} \leq \Theta}^{d, \varrho}$ by $\mathrm{P}_{\mathrm{C}^{m}, \alpha}^{d, \varrho} \leq \Theta$ because sequential compactness as stated in Lemma A.7 was the only property of this space used.

For $\mathrm{T}_{\mathrm{C}^{m^{\prime}, \alpha^{\prime}} \leq \Theta^{\prime}}^{\mathrm{k}, \mathrm{P}} \underset{\mathcal{M}_{\mathrm{C}}^{m, \alpha} \leq \Theta}{d, \varrho\left[k^{\prime}, C\right]}$ the proof of Lemma 1.29 applies mutatis mutandis by replacing the space $\mathrm{T}_{\mathrm{C}^{\mathrm{m}^{\prime}, \alpha^{\prime}} \leq \Theta^{\prime}}^{\mathrm{k}, 1} \mathrm{P}_{\mathcal{M}_{\mathrm{C}}^{m, \alpha} \leq \Theta}^{d, \varrho}$ with $\mathrm{T}_{\mathrm{C}^{\mathrm{m}^{\prime}, \alpha^{\prime}} \leq \Theta^{\prime}}^{\mathrm{k}, 1} \mathrm{P} \underset{\mathcal{M}_{\mathrm{C}}^{m, \alpha} \leq \Theta}{d, \varrho\left[k^{\prime}, C\right]}$.

Lemma A.8. Let $M_{n} \rightarrow M$ be a sequence in

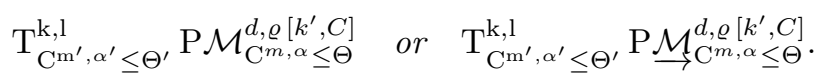

convergent in the sense of Definition 1.30. There are partially defined comparison maps $\iota_{n}: M_{n} \rightarrow M$ such that $\iota_{n *} \mu_{n i}$ weak ${ }^{\#}$ converges to $\mu_{i}$, moreover, for any $r>0$, and sufficiently large $n$ the image of $\iota_{n}$ contains $\mathrm{B}(p, r)$, and for some charts the tensors $\iota_{n}^{*} g_{n}$ and $\iota_{n}^{*} s_{n}$, resp., converge in $\mathrm{C}^{m, \alpha}$ to $g$ and $s$, resp, on these chart.

Proof. First look ar the case $\mathrm{T}_{\mathrm{C}^{m^{\prime}, \alpha^{\prime}} \leq \Theta^{\prime}}^{\mathrm{k}, 1} \mathrm{P} \mathcal{M}_{\mathrm{C}^{m}, \alpha}^{d, \varrho\left[k^{\prime}, C\right]}$. We can fix a countable atlas $\left\{U_{i}\right\}_{i \in \mathbb{N}}$ of $M$ such that any ball $\mathrm{B}(p, r)$ is covered by a finite number of charts. Thereby we can define a norm $\|$.$\| on \mathrm{C}^{m^{\prime}, \alpha^{\prime}}\left(M, \mathrm{~T}^{k, l} M\right)$ by

$$
\|(g, s)\|:=\sum_{i \in \mathbb{N}} 2^{-i}\left(\left\|\left.g\right|_{U_{i}}\right\|_{\mathrm{C}^{m, \alpha}}+\left\|\left.s\right|_{U_{i}}\right\|_{\mathrm{C}^{m^{\prime}, \alpha^{\prime}}}\right) .
$$

Moreover the space $\mathcal{M}(M)$ of measures on $M$ is metrizable DV08, Proposition 9.1.IV(i)]. Choose such a metric $d_{\mathcal{M} M}$.

For any $r$ the are partially defined comparison maps $\iota_{n}: M_{n}$ whose domain contains $\mathrm{B}\left(p_{n}, r\right)$ such that the measures $b_{p_{n}, r} \mu_{n i}$ converge to $b_{p, r} \mu_{i}$ as $n \rightarrow \infty$ for all $i=1, \ldots k^{\prime}$. For each $r=1,2, \ldots$ and sufficiently large $n \geq n_{r}$, we can find a comparison map $\iota_{r, n}: M_{n} \rightarrow M$ with distortion at most $\varepsilon$ such that $d_{\mathcal{M} M}\left(\iota_{r, n *}\left(b_{p_{n}, r} \mu_{i n}\right), b_{p, r} \mu_{i}\right)<r^{-1}$ and $\left\|\left(\iota_{n *} g_{n}, \iota_{n *} s_{n}\right)-(g, s)\right\|<r^{-1}$. We define a diagonal sequence by $\iota_{n}=\iota_{r(n), n}$ where $r(n)$ is the largest integer such that $\iota_{r, n}$ exists. By triangle inequality for $d_{\mathcal{M} M}$ and $\|\cdot\|$ the sequence $\iota_{n *}\left(b_{p_{n}, r} \mu_{i n}\right)$ weak ${ }^{\#}$ converges to $\mu_{i}$.

Since for any test function with bounded support, we can choose $\bar{r}$ so large that it contains the support of $f$, and hence

$$
\int f \mathrm{~d} \iota_{n *}\left(b_{p_{n}, \bar{r}} \mu_{i n}\right)=\int f \mathrm{~d} \iota_{n *}\left(b_{p_{n}, r(n)} \mu_{i n}\right)
$$

for sufficiently large $n$ and all $i=1, \ldots, k^{\prime}$. The claim for $\mathrm{T}_{\mathrm{C}^{m^{\prime}, \alpha^{\prime}} \leq \Theta^{\prime}}^{\mathrm{k}, 1} \mathrm{PM}_{\mathrm{C}^{m, \alpha} \leq \Theta}^{d, \varrho\left[k^{\prime}, C\right]}$ follows.

For $\mathrm{T}_{\mathrm{C}^{m^{\prime}, \alpha^{\prime}} \leq \Theta^{\prime}}^{\mathrm{k}, \mathrm{P}} \stackrel{\mathcal{M}_{\mathrm{C}^{m, \alpha} \leq \Theta}^{d, \varrho\left[k^{\prime}, C\right]}}{\mathrm{m}}$ do the same argument but with a representative of the orientations on the $\bar{M}_{n}$ 's and $M$. 


\section{Appendix B}

\section{Coordinate independence of rough curvature tensors}

In this appendix we check that the curvature defined in the notation of $\S 2.2 .1$ by the formula

$$
\begin{array}{r}
{ }^{\varphi} R_{\lambda \mu \nu}^{k}=\sum_{i \in I} \lambda_{i} \sum_{l}\left(\left(x_{k, l}^{i} \circ y^{i}\right)_{, \mu} y_{l, \nu \lambda}^{i}\right)_{[\mu \nu]}+\left(\lambda_{i, \mu}{ }^{i} \Gamma_{\nu \lambda}^{k}\right)_{[\mu \nu]} \\
+\sum_{\kappa}\left({ }^{\varphi} \Gamma_{\mu \kappa}^{k}{ }^{\varphi} \Gamma_{\nu \lambda}^{\kappa}\right)_{[\mu \nu]}
\end{array}
$$

is actually coordinate independent. The standard textbook calculation could be referred to if a third derivative of transition maps would exists.

Lemma 2.12. Given a cover of a manifold $M$ by charts $\varphi_{i}: U_{i} \rightarrow M$ in the $\mathrm{C}^{2}$-atlas of $M$ with a corresponding $\mathrm{C}^{1}$-partition of unity $\lambda_{i}$. For any two charts $\varphi: U \rightarrow M$ and $\varphi^{\prime}: U^{\prime} \rightarrow M$ the expression defined by (2.8) coincide on $\varphi(U) \cap \varphi^{\prime}\left(U^{\prime}\right)$ as (3,1)-tensor, i.e.

$$
\sum_{\lambda^{\prime}, \mu^{\prime}, \nu^{\prime}, k^{\prime}}\left(\varphi^{\prime} R_{\lambda^{\prime} \mu^{\prime} \nu^{\prime}}^{k^{\prime}} \circ x^{\prime}\right) x_{\lambda^{\prime}, \lambda}^{\prime} x_{\mu^{\prime}, \mu}^{\prime} x_{\nu^{\prime}, \nu}^{\prime}\left(x_{k, k^{\prime}} \circ x^{\prime}\right)={ }^{\varphi} R_{\lambda \mu \nu}^{k} .
$$

Proof. We repeat all crucial definitions in the primed and non-primed versions

$$
\begin{aligned}
x & =\varphi^{-1} \circ \varphi^{\prime}: U^{\prime} \rightarrow U \subset \mathbb{R}^{d}, & x^{\prime} & =\varphi^{-1} \circ \varphi: U \rightarrow U^{\prime} \subset \mathbb{R}^{d}, \\
x^{i} & =\varphi^{-1} \circ \varphi_{i}, & x^{\prime i} & =\varphi^{\prime-1} \circ \varphi_{i}, \\
y^{i} & =\varphi_{i}^{-1} \circ \varphi, & y^{\prime i} & =\varphi_{i}^{\prime-1} \circ \varphi^{\prime}, \\
{ }^{i} \Gamma_{\mu \nu}^{k} & =\sum_{l}\left(x_{k, l}^{i} \circ y^{i}\right)\left(y_{l, \mu \nu}^{i}\right), & { }^{i} \Gamma_{\mu \nu}^{\prime k} & =\sum_{l}\left(x_{k, l}^{i} \circ y^{\prime i}\right)\left(y_{l, \mu \nu}^{\prime i}\right), \\
{ }^{\varphi} \Gamma_{\mu \nu}^{k} & =\sum_{i \in I} \lambda_{i}{ }^{i} \Gamma_{\mu \nu}^{k}, & \varphi^{\prime} \Gamma_{\mu \nu}^{k} & =\sum_{i \in I} \lambda_{i}{ }^{i} \Gamma_{\mu \nu}^{\prime k} .
\end{aligned}
$$

defined on the suitable domains. Additionally, we introduce the shorthand

$$
\left(h_{i i^{\prime} j j^{\prime}}\right)_{\left[i^{(\prime)} j^{(\prime)}\right]}:=h_{i i^{\prime} j j^{\prime}}-h_{j j^{\prime} i i^{\prime}} .
$$


Observe that in case $h_{i i^{\prime} j j^{\prime}}=h_{i i^{\prime}}^{\prime} \cdot h_{j j^{\prime}}^{\prime \prime}$

$$
\begin{aligned}
\sum_{i^{\prime} j^{\prime}}\left(h_{i i^{\prime} j j^{\prime}}\right)_{\left[i^{(\prime)} j^{(\prime)}\right]} & =\left(\sum_{i^{\prime}} h_{i i^{\prime}}^{\prime}\right)\left(\sum_{j^{\prime}} h_{j j^{\prime}}^{\prime \prime}\right)-\left(\sum_{j^{\prime}} h_{j j^{\prime}}^{\prime}\right)\left(\sum_{i^{\prime}} h_{i i^{\prime}}^{\prime \prime}\right) \\
& =\left(\sum_{i^{\prime} j^{\prime}} h_{i i^{\prime} j j^{\prime}}\right)_{[i j]} .
\end{aligned}
$$

We have $x^{\prime}:=\varphi^{\prime-1} \circ \varphi=x^{i} \circ y^{i}$ and $x:=\varphi^{-1} \circ \varphi^{\prime}=x^{i} \circ y^{i}$ if defined Observe that for the differential $D$ id: $\mathbb{R}^{d} \rightarrow \mathbb{R}^{d}$ of the identity the following identity holds

$$
\begin{aligned}
0 & =(D \mathrm{id})_{, \lambda}=\left(\mathrm{D} x \circ x^{\prime}\right)_{, \lambda}=\left(\left(\sum_{l} x_{k, l} \circ x^{\prime} \cdot x_{l, \nu}^{\prime}\right)_{k \nu}\right)_{, \lambda} \\
& =\sum_{l}\left(\left(x_{k, l} \circ x^{\prime}\right)_{, \lambda} x_{l, \nu}^{\prime}+\left(x_{k, l} \circ x^{\prime}\right) x_{l, \nu \lambda}^{\prime}\right)_{k \nu} .
\end{aligned}
$$

We check

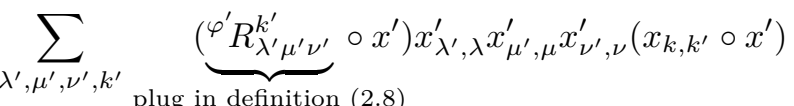

$$
\begin{aligned}
& =\sum_{\substack{i \in I \\
\lambda^{\prime}, k^{\prime}, l^{\prime}}} \lambda_{i} \underbrace{\sum_{\mu^{\prime}, \nu^{\prime}}\left(\begin{array}{r}
\left(\left(x_{k^{\prime}, l^{\prime}}^{i} \circ y^{\prime i}\right)_{, \mu^{\prime}} \circ x^{\prime}\right) x_{\mu^{\prime}, \mu}^{\prime} \\
\left(y_{l^{\prime}, \nu^{\prime} \lambda^{\prime}}^{\prime i} \circ x^{\prime}\right) x_{\lambda^{\prime}, \lambda}^{\prime} x_{\nu^{\prime}, \nu}^{\prime}\left(x_{k, k^{\prime}} \circ x^{\prime}\right)
\end{array}\right)_{\left[\mu^{(\prime)} \nu^{(\prime)}\right]}}_{\text {use formula }} \\
& +\sum_{\substack{i \in I \\
\lambda^{\prime}, k^{\prime}}} \underbrace{\sum_{\mu^{\prime}, \nu^{\prime}}\left(\lambda_{i, \mu^{\prime}} x_{\mu^{\prime}, \mu}^{\prime}{ }^{i} \Gamma_{\nu^{\prime} \lambda^{\prime}}^{\prime x^{\prime}} x_{\lambda^{\prime}, \lambda}^{\prime} x_{\nu^{\prime}, \nu}^{\prime}\left(x_{k, k^{\prime}} \circ x^{\prime}\right)\right)_{\left[\mu^{(\prime)} \nu^{(\prime)}\right]}}_{\text {use formula }}
\end{aligned}
$$

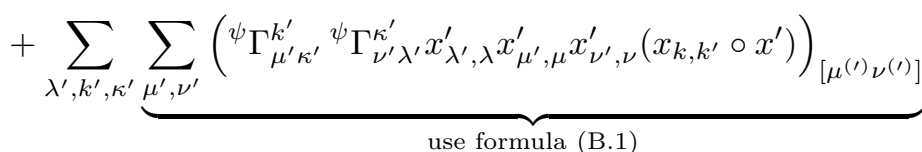

$$
\begin{aligned}
& =\sum_{\substack{i \in I \\
\lambda^{\prime}, k^{\prime}, l^{\prime}}} \lambda_{i}\left(\begin{array}{l}
\underbrace{\sum_{\mu^{\prime}}\left(\left(x_{k^{\prime}, l^{\prime}}^{i} \circ y^{\prime i}\right)_{, \mu^{\prime}} \circ x^{\prime}\right) x_{\mu^{\prime}, \mu}^{\prime}}_{\text {change of variables }} \\
\cdot \sum_{\nu^{\prime}} \underbrace{\left(y_{l^{\prime}, \nu^{\prime} \lambda^{\prime}}^{i} \circ x^{\prime}\right) x_{\lambda^{\prime}, \lambda}^{\prime}}_{\text {change of variables }} x_{\nu^{\prime}, \nu}^{\prime}\left(x_{k, k^{\prime}} \circ x^{\prime}\right)
\end{array}\right)_{[\mu \nu]} \\
& +\sum_{\substack{i \in I \\
\lambda^{\prime}, k^{\prime}}}(\underbrace{\sum_{\mu^{\prime}} \lambda_{i, \mu^{\prime}} x_{\mu^{\prime}, \mu}^{\prime}}_{\text {change of variables }} \underbrace{\sum_{\nu^{\prime}}^{i} \Gamma_{\nu^{\prime} \lambda^{\prime}}^{k^{\prime}} x_{\lambda^{\prime}, \lambda}^{\prime} x_{\nu^{\prime}, \nu}^{\prime}\left(x_{k, k^{\prime}} \circ x^{\prime}\right)}_{\text {apply formula }})_{[\mu \nu]} \\
& +\sum_{\lambda^{\prime}, k^{\prime}, \kappa^{\prime}}\left(\sum_{\mu^{\prime}}{ }^{\lambda^{\prime}} \Gamma_{\mu^{\prime} \kappa^{\prime}}^{k^{\prime}} x_{\mu^{\prime}, \mu}^{\prime}\left(x_{k, k^{\prime}} \circ x^{\prime}\right) \sum_{\nu^{\prime}} \psi_{\Gamma_{\nu^{\prime} \lambda^{\prime}}}^{\kappa^{\prime}} x_{\lambda^{\prime}, \lambda}^{\prime} x_{\nu^{\prime}, \nu}^{\prime}\right)_{[\mu \nu]} \\
& =\sum_{\substack{i \in I \\
k^{\prime}, l^{\prime}}} \lambda_{i}\left((\underbrace{x_{k^{\prime}, l^{\prime}}^{i}}_{=\left(x^{\prime} \circ x^{i}\right)_{k^{\prime}, l^{\prime}}} \circ \underbrace{y^{\prime i} \circ x^{\prime}}_{=y^{i}})_{, \mu} \sum_{\nu^{\prime}}(\underbrace{y_{l^{\prime}, \nu^{\prime}}^{i i}}_{=\left(y^{i} \circ x\right)_{l^{\prime}, \nu^{\prime}}} \circ x^{\prime})_{, \lambda} x_{\nu^{\prime}, \nu}^{\prime}\left(x_{k, k^{\prime}} \circ x^{\prime}\right)\right)_{[\mu \nu]} \\
& +\sum_{i \in I}\left(\lambda_{i, \mu}\left({ }^{i} \Gamma_{\nu \lambda}^{k}-\sum_{l}\left(x_{k, l} \circ x^{\prime}\right)\left(x_{l, \nu \lambda}^{\prime}\right)\right)\right)_{[\mu \nu]} \\
& +(\sum_{\substack{\lambda^{\prime}, k^{\prime}, \kappa^{\prime}, \mu^{\prime}, \nu^{\prime}, m}} \Gamma_{\mu^{\prime} m}^{k^{\prime}} x_{\mu^{\prime}, \mu}^{\prime}\left(x_{k, k^{\prime}} \circ x^{\prime}\right) \cdot \underbrace{\delta_{m \kappa^{\prime}}}_{\delta_{m \kappa^{\prime}} \circ x^{\prime}=(D \text { id })_{m \kappa^{\prime}} \circ x^{\prime}=\left(D\left(x^{\prime} \circ x\right)\right)_{m \kappa^{\prime}} \circ x^{\prime}} \cdot{ }^{\psi} \Gamma_{\nu^{\prime} \lambda^{\prime}}^{\kappa^{\prime}} x_{\lambda^{\prime}, \lambda}^{\prime} x_{\nu^{\prime}, \nu}^{\prime})_{[\mu \nu]} \\
& =\left(\sum_{\kappa} x_{m, \kappa}^{\prime} \circ x \cdot x_{\kappa, \kappa^{\prime}}\right) \circ x^{\prime}=\sum_{\kappa} x_{m, \kappa}^{\prime}\left(x_{\kappa, \kappa^{\prime}} \circ x^{\prime}\right)
\end{aligned}
$$




$$
\begin{aligned}
& =\sum_{\substack{i \in I \\
k^{\prime}, l^{\prime}}} \lambda_{i}\left(\begin{array}{l}
\left(\left(\sum_{\kappa} x_{k^{\prime}, \kappa}^{\prime} \circ x^{i} \cdot x_{\kappa, l^{\prime}}^{i}\right) \circ y^{i}\right)_{, \mu} \\
\cdot \sum_{\nu^{\prime}}\left(\left(\sum_{l} y_{l^{\prime}, l}^{i} \circ x \cdot x_{l, \nu^{\prime}}\right) \circ x^{\prime}\right)_{, \lambda} \cdot x_{\nu^{\prime}, \nu}^{\prime}\left(x_{k, k^{\prime}} \circ x^{\prime}\right)
\end{array}\right)_{[\mu \nu]} \\
& +\sum_{i \in I}\left(\lambda_{i, \mu}^{i} \Gamma_{\nu \lambda}^{k}\right)_{[\mu \nu]}-\sum_{l}\left(\left(\sum_{i \in I} \lambda_{i}\right)_{, \mu}\left(x_{k, l} \circ x^{\prime}\right)\left(x_{l, \nu \lambda}^{\prime}\right)\right)_{[\mu \nu]}
\end{aligned}
$$

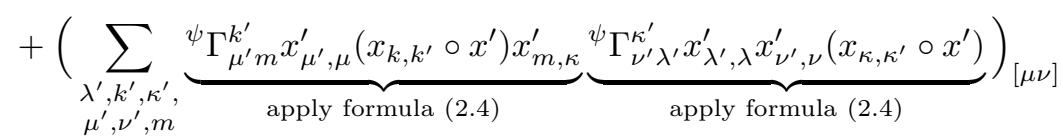

$$
\begin{aligned}
& =\sum_{\substack{i \in I \\
k^{\prime}, l^{\prime}}} \lambda_{i}\left(\sum_{\kappa}\left(x_{k^{\prime}, \kappa}^{\prime} \cdot x_{\kappa, l^{\prime}}^{i} \circ y^{i}\right)_{, \mu} \sum_{\nu, l}\left(y_{l^{\prime}, l}^{i} \cdot x_{l, \nu^{\prime}} \circ x^{\prime}\right)_{, \lambda} x_{\nu^{\prime}, \nu}^{\prime}\left(x_{k, k^{\prime}} \circ x^{\prime}\right)\right)_{[\mu \nu]} \\
& +\sum_{i \in I}\left(\lambda_{i, \mu}^{i} \Gamma_{\nu \lambda}^{k}\right)_{[\mu \nu]}-\sum_{l}\left(1_{, \mu}\left(x_{k, l} \circ x^{\prime}\right) x_{l, \nu \lambda}^{\prime}\right)_{[\mu \nu]} \\
& +\sum_{\kappa}\left(\left({ }^{\varphi} \Gamma_{\mu \kappa}^{k}-\sum_{l}\left(x_{k, l} \circ x^{\prime}\right) x_{l, \mu \kappa}^{\prime}\right)\left({ }^{\varphi} \Gamma_{\nu \lambda}^{\kappa}-\sum_{l}\left(x_{\kappa, l} \circ x^{\prime}\right) x_{l, \nu \lambda}^{\prime}\right)\right)_{[\mu \nu]} \\
& =\sum_{\substack{i \in I \\
k^{\prime}, l^{\prime}}} \lambda_{i}\left(\begin{array}{c}
\sum_{\kappa}\left(x_{k^{\prime}, \kappa \mu}^{\prime}\left(x_{\kappa, l^{\prime}}^{i} \circ y^{i}\right)+x_{k^{\prime}, \kappa}^{\prime}\left(x_{\kappa, l^{\prime}}^{i} \circ y^{i}\right)_{, \mu}\right) \\
\cdot \sum_{\nu^{\prime}, l}\left(y_{l^{\prime}, l \lambda}^{i}\left(x_{l, \nu^{\prime}} \circ x^{\prime}\right)+y_{l^{\prime}, l}^{i}\left(x_{l, \nu^{\prime}} \circ x^{\prime}\right)_{, \lambda}\right) x_{\nu^{\prime}, \nu}^{\prime}\left(x_{k, k^{\prime}} \circ x^{\prime}\right)
\end{array}\right)_{[\mu \nu]} \\
& +\sum_{i \in I}\left(\lambda_{i, \mu}{ }^{i} \Gamma_{\nu \lambda}^{k}\right)_{\left[\mu^{(\prime)} \nu^{(\prime)}\right]}-\sum_{l}\left(0 \cdot\left(x_{k, l} \circ x^{\prime}\right) x_{l, \nu \lambda}^{\prime}\right)_{[\mu \nu]} \\
& +\sum_{\kappa}\left(\begin{array}{c}
{ }^{\varphi} \Gamma_{\mu \kappa}^{k}{ }^{\varphi} \Gamma_{\nu \lambda}^{\kappa}+\sum_{l, l^{\prime}}-{ }^{\varphi} \Gamma_{\mu \kappa}^{k}\left(x_{\kappa, l} \circ x^{\prime}\right) x_{l, \nu \lambda}^{\prime} \\
-{ }^{\varphi} \Gamma_{\nu \lambda}^{\kappa}\left(x_{k, l} \circ x^{\prime}\right) x_{l, \mu \kappa}^{\prime}+\left(x_{k, l} \circ x^{\prime}\right) x_{l, \mu \kappa}^{\prime} \underbrace{\left(x_{\kappa, l^{\prime}} \circ x^{\prime}\right) x_{l^{\prime}, \nu \lambda}^{\prime}}_{\text {use formula }[\text { B.2. }}
\end{array}\right) \\
& =\sum_{\substack{i \in I \\
l, \kappa}} \lambda_{i}\left(\begin{array}{c}
\sum_{k^{\prime}, l^{\prime}}\left(x_{k^{\prime}, \kappa \mu}^{\prime}\left(x_{\kappa, l^{\prime}}^{i} \circ y^{i}\right)+x_{k^{\prime}, \kappa}^{\prime}\left(x_{\kappa, l^{\prime}}^{i} \circ y^{i}\right)_{, \mu}\right) \\
\cdot \sum_{\nu}\left(y_{l^{\prime}, l \lambda}^{i}\left(x_{l, \nu^{\prime}} \circ x^{\prime}\right)+y_{l^{\prime}, l}^{i}\left(x_{l, \nu^{\prime}} \circ x^{\prime}\right)_{, \lambda}\right) x_{\nu^{\prime}, \nu}^{\prime}\left(x_{k, k^{\prime}} \circ x^{\prime}\right)
\end{array}\right)_{[\mu \nu]} \\
& +\sum_{i \in I}\left(\lambda_{i, \mu}^{i} \Gamma_{\nu \lambda}^{k}\right)_{[\mu \nu]}+\sum_{\kappa}^{\varphi} \Gamma_{\mu \kappa}^{k}{ }^{\varphi} \Gamma_{\nu \lambda}^{\kappa}
\end{aligned}
$$

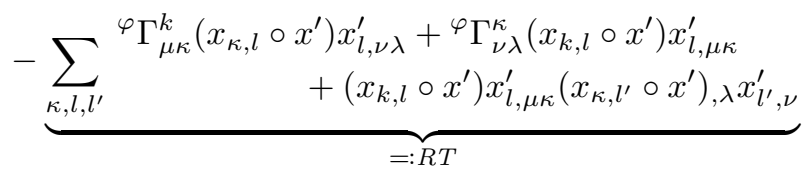

$$
\begin{aligned}
& =\sum_{i \in I,} \lambda_{i}\left(\begin{array}{l}
\sum_{k^{\prime}} x_{k^{\prime}, \kappa \mu}^{\prime}\left(x_{k, k^{\prime}} \circ x^{\prime}\right) \sum_{l^{\prime}}\left(x_{\kappa, l^{\prime}}^{i} \circ y^{i}\right) y_{l^{\prime}, l \lambda}^{i} \sum_{\nu^{\prime}}\left(x_{l, \nu^{\prime}} \circ x^{\prime}\right) x_{\nu^{\prime}, \nu}^{\prime} \\
+\sum_{l^{\prime}}\left(x_{\kappa, l^{\prime}}^{i} \circ y^{i}\right)_{, \mu} y_{l^{\prime}, l \lambda}^{i} \sum_{k^{\prime}} x_{k^{\prime}, \kappa}^{\prime}\left(x_{k, k^{\prime}} \circ x^{\prime}\right) \sum_{\nu^{\prime}}\left(x_{l, \nu^{\prime}} \circ x^{\prime}\right) x_{\nu^{\prime}, \nu}^{\prime} \\
+\sum_{k^{\prime}, \nu^{\prime}} x_{k^{\prime}, \kappa \mu}^{\prime}\left(x_{l, \nu^{\prime}} \circ x^{\prime}\right)_{, \lambda} x_{\nu^{\prime}, \nu}^{\prime}\left(x_{k, k^{\prime}} \circ x^{\prime}\right) \sum_{l^{\prime}}\left(x_{\kappa, l^{\prime}}^{i} \circ y^{i}\right) y_{l^{\prime}, l}^{i} \\
+\sum_{l^{\prime}, \nu^{\prime}} \underbrace{\left(x_{\kappa, l^{\prime}}^{i} \circ y^{i}\right)_{, \mu} y_{l^{\prime}, l}^{i}\left(x_{l, \nu^{\prime}} \circ x^{\prime}\right)_{, \lambda} x_{\nu^{\prime}, \nu}^{\prime}}_{\text {use formula }[\text { B.2] }} \sum_{k_{k^{\prime}}}\left(x_{k, k^{\prime}} \circ x^{\prime}\right) x_{k^{\prime}, \kappa}^{\prime}
\end{array}\right)_{[\mu \nu]}
\end{aligned}
$$$$
+\sum_{i \in I}\left(\lambda_{i, \mu}^{i} \Gamma_{\nu \lambda}^{k}\right)_{[\mu \nu]}+\sum_{\kappa}^{\varphi} \Gamma_{\mu \kappa}^{k} \Gamma_{\nu \lambda}^{\kappa}-R T
$$ 


$$
\begin{aligned}
& =\sum_{i \in I} \lambda_{i}\left(\begin{array}{c}
\sum_{k^{\prime}} x_{k^{\prime}, \kappa \mu}^{\prime}\left(x_{k, k^{\prime}} \circ x^{\prime}\right){ }^{i} \Gamma_{l \lambda}^{\kappa} \delta_{l \nu} \\
+\sum_{l^{\prime}}\left(x_{\kappa, l^{\prime}}^{i} \circ y^{i}\right)_{, \mu} y_{l^{\prime}, l \lambda}^{i} \delta_{\kappa k} \delta_{l \nu} \\
+\sum_{k^{\prime}, \nu^{\prime}} x_{k^{\prime}, \kappa \mu}^{\prime}\left(x_{l, \nu^{\prime}} \circ x^{\prime}\right)_{, \lambda} x_{\nu^{\prime}, \nu}^{\prime}\left(x_{k, k^{\prime}} \circ x^{\prime}\right) \delta_{\kappa l} \\
+\sum_{l^{\prime}, \nu^{\prime}}\left(x_{\kappa, l^{\prime}}^{i} \circ y^{i}\right) y_{l^{\prime}, l \mu}^{i}\left(x_{l, \nu^{\prime}} \circ x^{\prime}\right) x_{\nu^{\prime}, \nu \lambda}^{\prime} \delta_{k \kappa}
\end{array}\right)_{[\mu \nu]} \\
& +\sum_{i \in I}\left(\lambda_{i, \mu}^{i} \Gamma_{\nu \lambda}^{k}\right)_{[\mu \nu]}+\sum_{\kappa}{ }^{\varphi} \Gamma_{\mu \kappa}^{k}{ }^{\varphi} \Gamma_{\nu \lambda}^{\kappa}-R T \\
& =\sum_{i \in I} \lambda_{i}\left(\begin{array}{c}
\sum_{k^{\prime}} x_{k^{\prime}, \kappa \mu}^{\prime}\left(x_{k, k^{\prime}} \circ x^{\prime}\right){ }^{i} \Gamma_{\nu \lambda}^{\kappa} \\
+\sum_{l^{\prime}}\left(x_{k, l^{\prime}}^{i} \circ y^{i}\right)_{, \mu} y_{l^{\prime}, \nu \lambda}^{i} \\
+\sum_{k^{\prime}, \nu^{\prime}} x_{k^{\prime}, \kappa \mu}^{\prime}\left(x_{\kappa, \nu^{\prime}} \circ x^{\prime}\right)_{, \lambda} x_{\nu^{\prime}, \nu}^{\prime}\left(x_{k, k^{\prime}} \circ x^{\prime}\right) \\
+\sum_{\nu^{\prime}}{ }^{i} \Gamma_{\mu l}^{k}\left(x_{l, \nu^{\prime}} \circ x^{\prime}\right) x_{\nu^{\prime}, \nu \lambda}^{\prime}
\end{array}\right)_{[\mu \nu]} \\
& +\sum_{i \in I}\left(\lambda_{i, \mu}^{i} \Gamma_{\nu \lambda}^{k}\right)_{[\mu \nu]}+\sum_{\kappa}^{\varphi} \Gamma_{\mu \kappa}^{k} \Gamma_{\nu \lambda}^{\kappa}-R T \\
& =\sum_{l, \kappa}\left(\begin{array}{c}
\sum_{k^{\prime}} x_{k^{\prime}, \kappa \mu}^{\prime}\left(x_{k, k^{\prime}} \circ x^{\prime}\right)^{\varphi} \Gamma_{\nu \lambda}^{\kappa} \\
+\sum_{l^{\prime}}\left(x_{k, l^{\prime}}^{i} \circ y^{i}\right)_{, \mu} y_{l^{\prime}, \nu \lambda}^{i} \\
+\sum_{k^{\prime}, \nu^{\prime}} x_{k^{\prime}, \kappa \mu}^{\prime}\left(x_{\kappa, \nu^{\prime}} \circ x^{\prime}\right)_{, \lambda} x_{\nu^{\prime}, \nu}^{\prime}\left(x_{k, k^{\prime}} \circ x^{\prime}\right) \\
+\sum_{\nu^{\prime}}{ }^{\varphi} \Gamma_{\mu l}^{k}\left(x_{l, \nu^{\prime}} \circ x^{\prime}\right) x_{\nu^{\prime}, \nu \lambda}^{\prime}
\end{array}\right)_{[\mu \nu]} \\
& +\sum_{i \in I}\left(\lambda_{i, \mu}^{i} \Gamma_{\nu \lambda}^{k}\right)_{[\mu \nu]}+\sum_{\kappa}^{\varphi} \Gamma_{\mu \kappa}^{k} \Gamma_{\nu \lambda}^{\kappa} \\
& \begin{aligned}
-\sum_{\kappa, l, l^{\prime}}{ }^{\varphi} \Gamma_{\mu \kappa}^{k}\left(x_{\kappa, l} \circ x^{\prime}\right) x_{l, \nu \lambda}^{\prime}+ & { }^{\varphi} \Gamma_{\nu \lambda}^{\kappa}\left(x_{k, l} \circ x^{\prime}\right) x_{l, \mu \kappa}^{\prime} \\
& +\left(x_{k, l} \circ x^{\prime}\right) x_{l, \mu \kappa}^{\prime}\left(x_{\kappa, l^{\prime}} \circ x^{\prime}\right)_{, \lambda} x_{l^{\prime}, \nu}^{\prime}
\end{aligned} \\
& =\sum_{i \in I} \lambda_{i} \sum_{l^{\prime}}\left(\left(x_{k, l^{\prime}}^{i} \circ y^{i}\right)_{, \mu} y_{l^{\prime}, \nu \lambda}^{i}\right)_{[\mu \nu]}+\left(\lambda_{i, \mu}{ }^{i} \Gamma_{\nu \lambda}^{k}\right)_{[\mu \nu]}+\sum_{\kappa}\left({ }^{\varphi} \Gamma_{\mu \kappa}^{k}{ }^{\varphi} \Gamma_{\nu \lambda}^{\kappa}\right)_{[\mu \nu]} \\
& ={ }^{\varphi} R_{\lambda \mu \nu}^{k} \text {. }
\end{aligned}
$$




\section{Bibliography}

[16] Measured Group Theory (August 28-September 3, 2016). Mathematisches Forschungsinstitut Oberwolfach. 2016.

[7s11] M. Abert et al. On the growth of Betti numbers of locally symmetric spaces. April 2011. arXiv: 1104.5559 [math.GR]

[7s16] M. Abert et al. On the growth of L2-invariants of locally symmetric spaces, II: exotic invariant random subgroups in rank one. December 2016. arXiv: 1612.09510 [math.GT].

[7s17] M. Abert et al. "On the growth of $L^{2}$-invariants for sequences of lattices in Lie groups". In: Annals of Mathematics 185 (3 2017), pp. 711-790. arXiv: 1210.2961 [math.RT].

[AB16] M. Abert and I. Biringer. Unimodular measures on the space of all Riemannian manifolds. Version 3. October 2016. arXiv: 1606.03360 [math.GT].

[Abé+16] M. Abért et al. "Matchings in Benjamini-Schramm convergent graph sequences". In: Transactions of the American Mathematical Society 368.6 (2016), pp. 4197-4218.

[AC92] M. T. Anderson and J. Cheeger. " $C^{\alpha}$-compactness for manifolds with Ricci curvature and injectivity radius bounded below". In: Journal of Differential Geometry 35.2 (1992), pp. 265-281.

[AGS08] L. Ambrosio, N. Gigli, and G. Savare. Gradient Flows: In Metric Spaces and in the Space of Probability Measures. Lectures in Mathematics. ETH Zürich. Birkhäuser Basel, 2008.

[AH15] M. Abért and T. Hubai. "Benjamini-Schramm convergence and the distribution of chromatic roots for sparse graphs". In: Combinatorica 35.2 (2015), pp. 127-151.

[AL07] D. Aldous and R. Lyons. "Processes on unimodular random networks". Version 5. In: Electronic Journal of Probability 12 (2007), pp. 1454-1508. arXiv: 0603062 [math.PR]

[And17] S. Andraus. Benjamini-Schramm convergence and limiting eigenvalue density of random matrices. February 2017. arXiv: 1702.01281 [math.PR].

[And90] M. T. Anderson. "Convergence and rigidity of manifolds under Ricci curvature bounds". In: Inventiones mathematicae 102.1 (1990), pp. $429-445$. 
[AS04] D. Aldous and J. M. Steele. "The objective method: probabilistic combinatorial optimization and local weak convergence". In: Probability on discrete structures. Springer, 2004, pp. 1-72.

[ATV17] M. Abért, A. Thom, and B. Virág. Benjamini-Schramm convergence and pointwise convergence of the spectral measure. October 9, 2017. URL: http://www.renyi.hu/ abert/luckapprox.pdf preprint.

[BBI01] D. Burago, Y. Burago, and S. Ivanov. A Course in Metric Geometry. Graduate Studies in Mathematics 33. Providence: American Mathematical Society, 2001.

[Ben+99] I. Benjamini et al. "Group-invariant percolation on graphs". In: Geometric and Functional Analysis 9.1 (1999), pp. 29-66.

[Bjö69] J.-E. Björk. "On extensions of Lipschitz functions". In: Arkiv för Matematik 7.6 (1969), pp. 513-515.

[BL10] C. Bordenave and M. Lelarge. "Resolvent of large random graphs". In: Random Structures \& Algorithms 37.3 (2010), pp. 332-352.

[Blo86] J. Blot. "The rank theorem in infinite dimension". In: Nonlinear Analysis. Theory, Methods \& Applications 10.10 (1986), pp. 10091020.

[Bor+13] C. Borgs et al. "Left and right convergence of graphs with bounded degree". In: Random Structures \& Algorithms 42.1 (2013), pp. 1-28.

[Bor16] C. Bordenave. Spectrum of random graphs. June 2016. URL: http: //www.math.univ-toulouse.fr/ bordenave/coursSRG.pdf.

[Bow15] L. Bowen. "Cheeger constants and $L^{2}$-Betti numbers". Version 4. In: Duke Mathematical Journal 164.3 (February 2015), pp. 569-615. arXiv: 1303.5963 [math.GT]

[Bow17] L. Bowen. Examples in the entropy theory of countable group actions. April 2017. arXiv: 1704.06349 [math.DS]

[BS01] I. Benjamini and O. Schramm. "Recurrence of Distributional Limits of Finite Planar Graphs". In: Electronic Journal of Probability 6.23 (2001), pp. 1-13.

[BSS08] I. Benjamini, O. Schramm, and A. Shapira. "Every minor-closed property of sparse graphs is testable". In: Proceedings of the fortieth annual ACM symposium on Theory of computing. ACM. 2008, pp. 393-402.

[Car05] Carothers. A Short Course on Banach Space Theory. London Mathematical Society St. Cambridge University Press, 2005.

[CC97] J. Cheeger and T. H. Colding. "On the structure of spaces with Ricci curvature bounded below. I". In: Journal of Differential Geometry 46.3 (1997), pp. 406-480.

[CDK11] G. Csató, B. Dacorogna, and O. Kneuss. The Pullback Equation for Differential Forms. Progress in Nonlinear Differential Equations and Their Applications 83. Boston: Birkhäuser, 2011. 
[CG85] J. Cheeger and M. Gromov. "On the Characteristic Numbers of Complete Manifolds of Bounded Curvature and Finite Volume". In: Differential Geometry and Complex Analysis. A Volume Dedicated to the Memory of Harry Ernest Rauch. Ed. by I. Chavel and H. M. Farkas. Berlin, Heidelberg: Springer, 1985, pp. 115-154.

[CH70] E. Calabi and P. Hartman. "On the smoothness of isometries". In: Duke Mathematical Journal 37.4 (December 1970), pp. 741-750.

[Che67] J. Cheeger. "Comparison and Finiteness Theorems for Riemannian Manifolds". PhD thesis. 1967, p. 75.

[Chr91] P. T. Chruściel. On uniqueness in the large of solutions of Einstein's equations ("strong cosmic censorship"). Proceedings of the Centre for Mathematics and its Applications 27. Canberra: Centre for Mathematics and its Applications, Australian National University, 1991.

[CN13] J. Cheeger and A. Naber. "Lower bounds on Ricci curvature and quantitative behavior of singular sets". In: Inventiones mathematicae (2013), pp. 1-19.

[CN15] J. Cheeger and A. Naber. "Regularity of Einstein manifolds and the codimension 4 conjecture". In: Annals of Mathematics 182 (3 2015), pp. 1093-1165.

[DFT03] B. Dacorogna, N. Fusco, and L. Tartar. "On the solvability of the equation $\operatorname{div} u=f$ in $L^{1}$ and in $C^{0}$ ". In: Rendiconti Lincei. Matematica e Applicazioni 14.3 (September 2003), pp. 239-245.

[Die69] J. A. E. Dieudonné. Treatise on analysis. 6 vols. Pure and Applied Mathematics 10. Academic Press Inc., 1969-1978.

[Dud02] R. M. Dudley. Real Analysis and Probability. Cambridge University Press, 2002.

[DV03] D. J. Daley and D. Vere-Jones. An Introduction to the Theory of Point Processes. Vol. 1: Elementary Theory and Methods. 2nd ed. An Introduction to the Theory of Point Processes. Springer, 2003.

[DV08] D. J. Daley and D. Vere-Jones. An Introduction to the Theory of Point Processes. Vol. 2: General Theory and Structure. 2nd ed. An Introduction to the Theory of Point Processes. Springer, 2008.

[Eic91] J. Eichhorn. "The Boundedness of Connection Coefficients and their Derivatives". In: Mathematische Nachrichten 152.1 (1991), pp. 145158.

[Ele10] G. Elek. "Betti Numbers are Testable". In: Fete of Combinatorics and Computer Science 20.6 (2010), pp. 564-631.

[Fed14] H. Federer. Geometric Measure Theory. Classics in Mathematics. Berlin, Heidelberg: Springer, 2014.

[FG97] B. E. Fristedt and L. F. Gray. A Modern Approach to Probability Theory. Probability and Its Applications. Birkhäuser, 1997.

[FN05] E. Fischer and I. Newman. "Testing versus estimation of graph properties". In: Proceedings of the 37th Annual ACM Symposium on Theory of Computing. 2005. 
[FP17] T. Fritz and P. Perrone. A Probability Monad as the Colimit of Finite Powers. December 2017. arXiv: 1712.05363 [math.PR].

[Fre03] D. H. Fremlin. Measure Theory. Vol. 4: Topological Measure Spaces. Torres Fremlin, 2003.

[Gel15] T. Gelander. A lecture on Invariant Random Subgroups. March 2015. arXiv: 1503.08402 [math.GR]

[Gir82] M. Giry. "A categorical approach to probability theory". In: Categorical Aspects of Topology and Analysis. Ed. by B. Banaschewski. Berlin, Heidelberg: Springer, 1982, pp. 68-85.

[GL17] T. Gelander and A. Levit. Invariant random subgroups over nonArchimedean local fields. Version 2. July 2017. arXiv: 1707.03578 [math.GR].

[Gol10] O. Goldreich. "Introduction to Testing Graph Properties". In: Property Testing: Current Research and Surveys. Ed. by O. Goldreich. Berlin, Heidelberg: Springer, 2010, pp. 105-141.

[GT77] D. Gilbarg and N. S. Trudinger. Elliptic partial differential equations of second order. Grundlehren der mathematischen Wissenschaften. Springer, 1977.

[Heb96] E. Hebey. Sobolev spaces on Riemannian manifolds. Lecture Notes in Mathematics 1635. Springer, 1996.

[Hir97] M. Hirsch. Differential Topology. Graduate Texts in Mathematics. New York: Springer, 1997.

[Hör12] L. Hörmander. The Analysis of Linear Partial Differential Operators. Vol. 1: Distribution Theory and Fourier Analysis. Grundlehren der mathematischen Wissenschaften. Berlin, Heidelberg: Springer, 2012.

[HW53] P. Hartman and A. Wintner. "On the Existence of Riemannian Manifolds which cannot Carry Non-Constant Analytic or Harmonic Functions in the Small". In: American Journal of Mathematics 75.2 (1953), pp. 260-276.

[Kel75] J. L. Kelley. General Topology. Graduate Texts in Mathematics. New York: Springer, 1975.

[KSS13] M. Kunzinger, R. Steinbauer, and M. Stojkovic. The exponential map of a $C^{1,1}$-metric. June 2013. arXiv: 1306.4776 [math.DG].

[Lan12] S. Lang. Fundamentals of Differential Geometry. Graduate Texts in Mathematics. New York: Springer, 2012.

[Les14] P. Lessa. "Brownian motion on stationary random manifolds". PhD thesis. Université Pierre et Marie Curie, Universidad de la República, 2014. URL: http://www. cmat . edu.uy/ lessa/resource/these pdf

[Les15] P. Lessa. "Reeb stability and the Gromov-Hausdorff limits of leaves in compact foliations". In: Asian journaltitle of Mathematics 19.3 (2015), pp. 433-463.

[Luc11] D. Luckhardt. "Random Homological Invariants of Simplicial Complexes". Unpublished. BA thesis. Georg-August-Universität Göttingen, September 2011. 
[Luc14] D. Luckhardt. "Random Metric Spaces and Testability". Unpublished. MA thesis. Georg-August-Universität Göttingen, September 2014.

[LY06] A. Lytchak and A. Yaman. "On Hölder continuous Riemannian and Finsler metrics". In: Transactions of the American Mathematical Society 358.7 (2006), pp. 2917-2926.

[Lyo05] R. Lyons. "Asymptotic enumeration of spanning trees". In: Combinatorics, Probability and Computing 14.4 (2005), pp. 491-522.

[Mal17] C. Male. Traffic distributions and independence: permutation invariant random matrices and the three notions of independence. May 2017. arXiv: 1111.4662 [math.PR].

[McS34] E. J. McShane. "Extension of range of functions". In: Bulletin of the American Mathematical Society 40.12 (1934).

[MS39] S. B. Myers and N. E. Steenrod. "The group of isometries of a Riemannian manifold". In: Annals of Mathematics (1939), pp. 400416.

[MS74] J. W. Milnor and J. D. Stasheff. Characteristic Classes. Annals of mathematics studies. Princeton University Press, 1974.

[MT16] V. S. Matveev and M. Troyanov. The Myers-Steenrod theorem for Finsler manifolds of low regularity. May 2016. arXiv: 1605.03850 [math.DG].

[Pet01] P. Pettersson. Differentiability of Minimal Geodesics in Metrics of Low Regularity. Mathematical Physics Preprint Archive, 2001. URL: http: //www.ma. utexas.edu/mp_arc-bin/mpa?yn=01-400.

[Pet16] P. Petersen. Riemannian Geometry. 3rd ed. Graduate Texts in Mathematics 171. Springer, 2016.

[PR14] B. Piccoli and F. Rossi. "Generalized Wasserstein Distance and its Application to Transport Equations with Source". In: Archive for Rational Mechanics and Analysis 211.1 (January 1, 2014), pp. 335358.

[PR16] B. Piccoli and F. Rossi. "On Properties of the Generalized Wasserstein Distance". In: Archive for Rational Mechanics and Analysis 222.3 (December 1, 2016), pp. 1339-1365.

[Pre97] D. Preiss. "Additional regularity for Lipschitz solutions of PDE". In: Journal für die reine und angewandte Mathematik 485 (1997), pp. 197-208.

[Rai13] J. Raimbault. On the convergence of arithmetic orbifolds. November 2013. arXiv: 1311.5375 [math.GT].

[Res78] Y. G. Reshetnyak. "Differential properties of quasiconformal maps and conformal maps of Riemannian spaces". In: Siberian Mathematical Journal 19.5 (1978), pp. 822-834.

[Rob91] S. M. Robinson. "An implicit-function theorem for a class of nonsmooth functions". In: Mathematics of operations research 16.2 (1991), pp. 292-309. 
[Ron10] X. Rong. "Convergence and Collapsing Theorems in Riemannian Geometry". In: Handbook of geometric analysis. Ed. by L. Ji et al. Vol. 2. Advanced Lectures in Mathematics 13. 2010.

[Sch71] H. H. Schaefer. Topological Vector Spaces. Graduate Texts in Mathematics. New York: Springer, 1971.

[Sch75] H. Schubert. Topologie. Eine Einführung. Mathematische Leitfäden. Stuttgart: B. G. Teubner, 1975.

[Tay00] M. E. Taylor. Tools for PDE. Pseudodifferential Operators, Paradifferential Operators, and Layer Potentials. Mathematical Surveys and Monographs 81. American Mathematical Society, 2000.

[Tay06] M. E. Taylor. "Existence and regularity of isometries". In: Transactions of the American Mathematical Society 358.6 (June 2006), pp. $2415-2423$.

[Tay07] M. E. Taylor. "Anderson-Cheeger limits of smooth Riemannian manifolds, and other Gromov-Hausdorff limits". In: The Journal of Geometric Analysis 17.2 (June 2007), pp. 365-374. 


\section{Erklärung gemäß $§ 8$ Absatz 2}

Ich versichere hiermit, dass ich die Dissertation selbstständig und ohne unerlaubte Hilfe angefertigt habe. Ich habe anderweitig keine Promotion beantragt, für die die hier vorgelegte Dissertation oder Teile hiervon eingereicht worden sind.

Daniel Luckhardt, Göttingen 24. April 2018 